\title{
Varying Constants, Gravitation and Cosmology
}

\author{
Jean-Philippe Uzan \\ Institut d'Astrophysique de Paris, \\ UMR-7095 du CNRS, Université Pierre et Marie Curie, \\ 98 bis bd Arago, 75014 Paris (France) \\ and \\ Department of Mathematics and Applied Mathematics, \\ Cape Town University, \\ Rondebosch 7701 (South Africa) \\ and \\ National Institute for Theoretical Physics (NITheP), \\ Stellenbosch 7600 (South Africa) \\ email: uzan@iap.fr \\ http://www2.iap.fr/users/uzan/
}

Accepted on 23 March 2011

Published on 29 March 2011

\begin{abstract}
Fundamental constants are a cornerstone of our physical laws. Any constant varying in space and/or time would reflect the existence of an almost massless field that couples to matter. This will induce a violation of the universality of free fall. Thus, it is of utmost importance for our understanding of gravity and of the domain of validity of general relativity to test for their constancy. We detail the relations between the constants, the tests of the local position invariance and of the universality of free fall. We then review the main experimental and observational constraints that have been obtained from atomic clocks, the Oklo phenomenon, solar system observations, meteorite dating, quasar absorption spectra, stellar physics, pulsar timing, the cosmic microwave background and big bang nucleosynthesis. At each step we describe the basics of each system, its dependence with respect to the constants, the known systematic effects and the most recent constraints that have been obtained. We then describe the main theoretical frameworks in which the low-energy constants may actually be varying and we focus on the unification mechanisms and the relations between the variation of different constants. To finish, we discuss the more speculative possibility of understanding their numerical values and the apparent fine-tuning that they confront us with.
\end{abstract}

This review is licensed under a Creative Commons Attribution-Non-Commercial-NoDerivs 3.0 Germany License. http://creativecommons.org/licenses/by-nc-nd/3.0/de/ 


\section{Imprint / Terms of Use}

Living Reviews in Relativity is a peer reviewed open access journal published by the Max Planck Institute for Gravitational Physics, Am Mühlenberg 1, 14476 Potsdam, Germany. ISSN 1433-8351.

This review is licensed under a Creative Commons Attribution-Non-Commercial-NoDerivs 3.0

Germany License: http://creativecommons.org/licenses/by-nc-nd/3.0/de/

Because a Living Reviews article can evolve over time, we recommend to cite the article as follows:

Jean-Philippe Uzan,

"Varying Constants, Gravitation and Cosmology",

Living Rev. Relativity, 14, (2011), 2. [Online Article]: cited [<date $>$ ], http://www.livingreviews.org/lrr-2011-2

The date given as $<$ date $>$ then uniquely identifies the version of the article you are referring to.

\section{Article Revisions}

Living Reviews supports two ways of keeping its articles up-to-date:

Fast-track revision A fast-track revision provides the author with the opportunity to add short notices of current research results, trends and developments, or important publications to the article. A fast-track revision is refereed by the responsible subject editor. If an article has undergone a fast-track revision, a summary of changes will be listed here.

Major update A major update will include substantial changes and additions and is subject to full external refereeing. It is published with a new publication number.

For detailed documentation of an article's evolution, please refer to the history document of the article's online version at http://www. livingreviews.org/lrr-2011-2. 


\section{Contents}

1 Introduction $\quad 7$

2 Constants and Fundamental Physics $\quad 9$

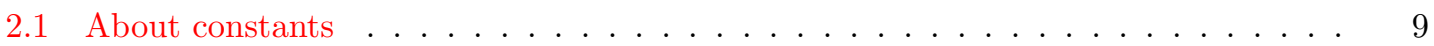

2.1.1 Characterizing the fundamental constants . . . . . . . . . . . . . 9

2.1 .2 Constants and metrology . . . . . . . . . . . . . . . . 13

2.2 The constancy of constants as a test of general relativity . . . . . . . . . . . . . 17

2.2.1 General relativity . . . . . . . . . . . . . . . . . . . . . . . . . . . . . . . . .

2.2.2 Varying constants and the universality of free fall . . . . . . . . . . . . 21

2.2.3 Relations with cosmology . . . . . . . . . . . . . . . . . . 22

3 Experimental and Observational Constraints 26

3.1 Atomic clocks . . . . . . . . . . . . . . . . . . . 26

3.1.1 Atomic spectra and constants . . . . . . . . . . . . . . . . 26

3.1 .2 Experimental constraints . . . . . . . . . . . . . . . . . . . . . . . . . . . .

3.1 .3 Physical interpretation . . . . . . . . . . . . . . . . 32

3.1 .4 Future evolutions . . . . . . . . . . . . . . . . . . . 33

3.2 The Oklo phenomenon . . . . . . . . . . . . . . . . . . . . 34

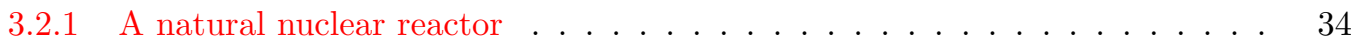

3.2.2 Constraining the shift of the resonance energy . . . . . . . . . . . . . 36

3.2.3 From the resonance energy to fundamental constants . . . . . . . . . . 38

3.3 Meteorite dating . . . . . . . . . . . . . . . . . . . . . . . . 40 40

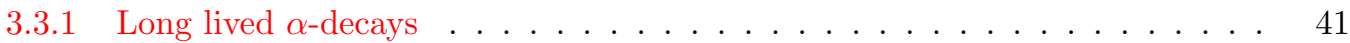

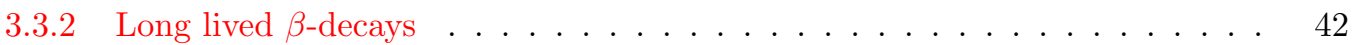

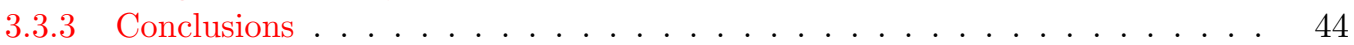

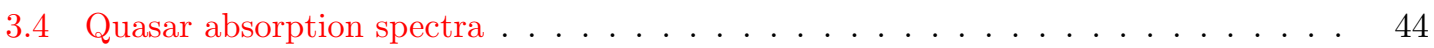

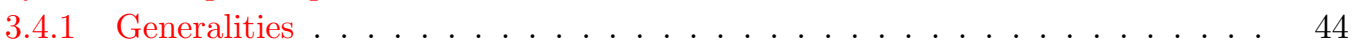

3.4 Alkali doublet method $(\mathrm{AD}) \ldots \ldots \ldots 4$

3.4.3 Many multiplet method $(\mathrm{MM}) \ldots \ldots$. . . . . . . . . . . . . . . . . . . . . . . . . 47

3.4.4 Single ion differential measurement (SIDAM) . . . . . . . . . . . . . 51

3.4.5 H I-21 cm vs. UV: $x=\alpha_{\mathrm{EM}}^{2} g_{\mathrm{p}} / \mu \ldots \ldots \ldots \ldots$

3.4.6 H I vs. molecular transitions: $y \equiv g_{\mathrm{p}} \alpha_{\mathrm{EM}}^{2} \ldots \ldots \ldots \ldots$. . . . . . . . . 52

$3.4 .7 \mathrm{OH}-18 \mathrm{~cm}: F=g_{\mathrm{p}}\left(\alpha_{\mathrm{EM}}^{2} \mu\right)^{1.57 \ldots \ldots \ldots \ldots \ldots \ldots} 53$

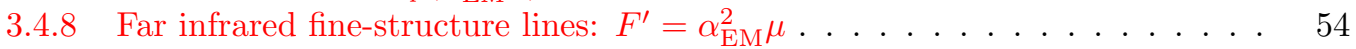

3.4 .9 "Conjugate" satellite $\mathrm{OH}$ lines: $G=g_{\mathrm{p}}\left(\alpha_{\mathrm{EM}} \mu\right)^{1.85} \ldots \ldots \ldots 4$

3.4.10 Molecular spectra and the electron-to-proton mass ratio . . . . . . . . 55

3.4.11 Emission spectra . . . . . . . . . . . . . . . . . 58

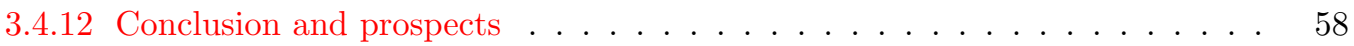

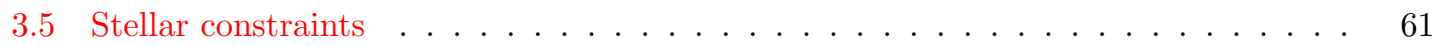

3.6 Cosmic Microwave Background . . . . . . . . . . . . . . . . . 63

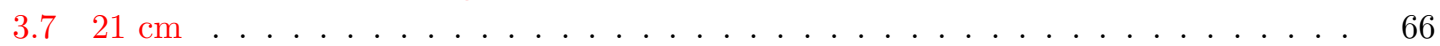

3.8 Big bang nucleosynthesis . . . . . . . . . . . . . . . . . . . . . . . . . . . . . . . . . . . . . 68

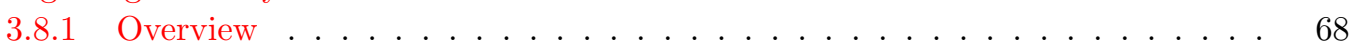

3.8 Constants everywhere. . . . . . . . . . . . . . . . . 70

3.8.3 From BBN parameters to fundamental constants . . . . . . . . . . . . . 73

3.8.4 Conclusion ........................ . . . 75 
4 The Gravitational Constant $\quad 76$

4.1 Solar systems constraints . . . . . . . . . . . . . . . . . . . 76

4.2 Pulsar timing . . . . . . . . . . . . . . . . . . . . . . . . . . . . . . . . . . . . .

4.3 Stellar constraints . . . . . . . . . . . . . . . . . . . 79

4.3.1 Ages of globular clusters . . . . . . . . . . . . . . . . . . 79

4.3.2 Solar and stellar seismology . . . . . . . . . . . . . . . . . . 80

4.3.3 Late stages of stellar evolution and supernovae . . . . . . . . . . . . . . . 81

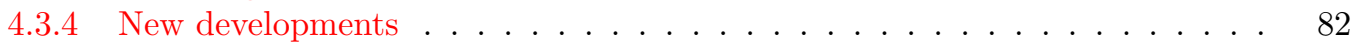

4.4 Cosmological constraints . . . . . . . . . . . . . . . . . . . . . 82

4.4 Cosmic microwave background . . . . . . . . . . . . . . . . . . 82

$4.4 \mathrm{BBN} \ldots \ldots \ldots \ldots 3$

5 Theories With Varying Constants $\quad 85$

5.1 Introducing new fields: generalities . . . . . . . . . . . . . . . . . . . 85

5.1 .1 The example of scalar-tensor theories . . . . . . . . . . . . 85

5.1 .2 Making other constants dynamical . . . . . . . . . . . . . . . . 87

5.2 High-energy theories and varying constants . . . . . . . . . . . . . . . . . . . 88

5.2 .1 Kaluza-Klein . . . . . . . . . . . . . . . . . . . 88

5.2 .2 String theory . . . . . . . . . . . . . . . . . . . 89

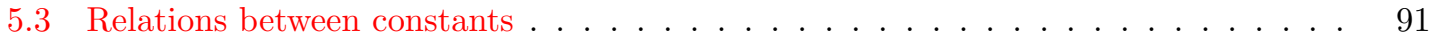

5.3.1 Implication of gauge coupling unification . . . . . . . . . . . . . . . . . . . . 92

5.3 .2 Masses and binding energies . . . . . . . . . . . . . . . . . . . . . . 94

5.3 .3 Gyromagnetic factors . . . . . . . . . . . . . . . . . . . . . 96

5.4 Models with varying constants . . . . . . . . . . . . . . . . . . 96

5.4.1 String dilaton and Runaway dilaton models . . . . . . . . . . . . . . . . . . . 97

5.4 .2 The Chameleon mechanism . . . . . . . . . . . . . . . . 99

5.4 Bekenstein and related models . . . . . . . . . . . . . . . . 100

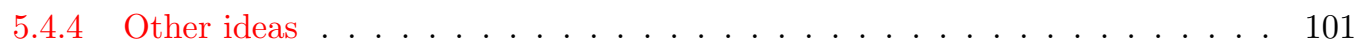

6 Spatial Variations 103

6.1 Local scales . . . . . . . . . . . . . . . . . . . . . . . . 103

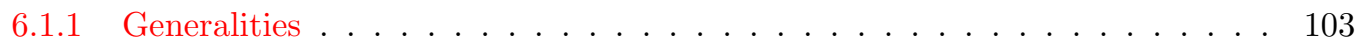

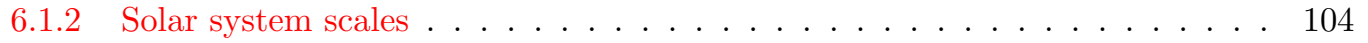

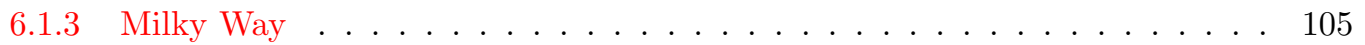

6.2 Cosmological scales . . . . . . . . . . . . . . . . . . . . 106

6.3 Implication for the universality of free fall . . . . . . . . . . . . . . . . . . 107

7 Why Are The Constants Just So? 110

7.1 Universe and multiverse approaches . . . . . . . . . . . . . . . . . . . 110

7.2 Fine-tunings and determination of the anthropic range . . . . . . . . . . . . . 111

7.3 Anthropic predictions . . . . . . . . . . . . . . . . . . . 112

8 Conclusions $r$

9 Acknowledgments $r 15$

A Notations $r$

A.1 Constants . . . . . . . . . . . . . . . . . . . 116

A.2 Sensitivity coefficients . . . . . . . . . . . . . . . . . . . . . . 116

A.3 Background cosmological spacetime . . . . . . . . . . . . . . . . . . 117 


\section{List of Tables}

1 List of the fundamental constants of our standard model. . . . . . . . . . . . . . 10

2 List of some related constants that appear in our discussions. See Ref. [379]. . . . 12

3 Summary of the constraints on the violation of the universality of free fall. . . . . . 19

4 Main cosmological parameters in the standard $\Lambda$-CDM model. . . . . . . . . . . . . 24

5 Summary of the systems considered to set constraints on the variation of the fundamental constants. . . . . . . . . . . . . . . . 26

6 Sensitivity of various transitions on a variation of the fine-structure constant. . . . 29

7 Summary of the constraints obtained from the comparisons of atomic clocks. . . . 29

8 Summary of the analysis of the Oklo data. . . . . . . . . . . . . . . . . . 37

9 Summary of the main nuclei and their physical properties that have been used in $\alpha$-decay studies. . . . . . . . . . . . . . . . . . . . 42

10 Summary of the latest constraints on the variation of fundamental constants obtained from the analysis of quasar absorption spectra. . . . . . . . . . . . . 59

11 Summary of the latest constraints on the variation of fundamental constants obtained from the analysis of cosmological data and more particularly of CMB data. 



\section{Introduction}

Fundamental constants appear everywhere in the mathematical laws we use to describe the phenomena of Nature. They seem to contain some truth about the properties of the physical world while their real nature seem to evade us.

The question of the constancy of the constants of physics was probably first addressed by Dirac [155, 156] who expressed, in his "Large Numbers hypothesis", the opinion that very large (or small) dimensionless universal constants cannot be pure mathematical numbers and must not occur in the basic laws of physics. He suggested, on the basis of this numerological principle, that these large numbers should rather be considered as variable parameters characterizing the state of the universe. Dirac formed five dimensionless ratios among which ${ }^{1} \delta \equiv H_{0} \hbar / m_{\mathrm{p}} c^{2} \sim 2 h \times 10^{-42}$ and $\epsilon \equiv G \rho_{0} / H_{0}^{2} \sim 5 h^{-2} \times 10^{-4}$ and asked the question of which of these ratios is constant as the universe evolves. Usually, $\delta$ varies as the inverse of the cosmic time while $\epsilon$ varies also with time if the universe is not described by an Einstein-de Sitter solution (i.e., when a cosmological constant, curvature or radiation are included in the cosmological model). Dirac then noticed that $\alpha_{\mathrm{G}} / \mu \alpha_{\mathrm{EM}}$, representing the relative magnitude of electrostatic and gravitational forces between a proton and an electron, was of the same order as $H_{0} e^{2} / m_{\mathrm{e}} c^{2}=\delta \alpha_{\mathrm{EM}} \mu$ representing the age of the universe in atomic units so that his five numbers can be "harmonized" if one assumes that $\alpha_{\mathrm{G}}$ and $\delta$ vary with time and scale as the inverse of the cosmic time.

This argument by Dirac is indeed not a physical theory but it opened many doors in the investigation on physical constants, both on questioning whether they are actually constant and on trying to understand the numerical values we measure.

First, the implementation of Dirac's phenomenological idea into a field-theory framework was proposed by Jordan [268], who realized that the constants have to become dynamical fields and proposed a theory where both the gravitational and fine-structure constants can vary ([497] provides a summary of some earlier attempts to quantify the cosmological implications of Dirac's argument). Fierz [195] then realized that in such a case, atomic spectra will be spacetime-dependent so that these theories can be observationally tested. Restricting to the sub-case in which only $G$ can vary led to definition of the class of scalar-tensor theories, which were further explored by Brans and Dicke [67]. This kind of theory was further generalized to obtain various functional dependencies for $G$ in the formalization of scalar-tensor theories of gravitation (see, e.g., [124]).

Second, Dicke [151] pointed out that in fact the density of the universe is determined by its age, this age being related to the time needed to form galaxies, stars, heavy nuclei... This led him to formulate that the presence of an observer in the universe places constraints on the physical laws that can be observed. In fact, what is meant by observer is the existence of (highly?) organized systems and this principle can be seen as a rephrasing of the question "why is the universe the way it is?" (see [252]). Carter [82, 83], who actually coined the term "anthropic principle" for it, showed that the numerological coincidences found by Dirac can be derived from physical models of stars and the competition between the weakness of gravity with respect to nuclear fusion. Carr and Rees [80] then showed how one can scale up from atomic to cosmological scales only by using combinations of $\alpha_{\mathrm{EM}}, \alpha_{\mathrm{G}}$ and $m_{\mathrm{e}} / m_{\mathrm{p}}$.

To summarize, Dirac's insight was to question whether some numerical coincidences between very large numbers, that cannot be themselves explained by the theory in which they appear, was a mere coincidence or whether it can reveal the existence of some new physical laws. This gives three main roads of investigation

- how do we construct theories in which what were thought to be constants are in fact dynamical fields,

${ }^{1} H_{0}$ is the Hubble constant today, $\rho_{0}$ the mean matter energy density today. The other constants are defined in Tables 1 and 2 and the definitions of the cosmological quantities are summarized in Appendix A.3. 
- how can we constrain, experimentally or observationally, the spacetime dependencies of the constants that appear in our physical laws

- how can we explain the values of the fundamental constants and the fine-tuning that seems to exist between their numerical values.

While "varying constants" may seem, at first glance, to be an oxymoron, it has to be considered merely as jargon to be understood as "revealing new degrees of freedom, and their coupling to the known fields of our theory". The tests on the constancy of the fundamental constants are indeed very important tests of fundamental physics and of the laws of Nature we are currently using. Detecting any such variation will indicate the need for new physical degrees of freedom in our theories, that is new physics.

The necessity of theoretical physics on deriving bounds on their variation is, at least, threefold:

1. it is necessary to understand and to model the physical systems used to set the constraints. In particular one needs to relate the effective parameters that can be observationally constrained to a set of fundamental constants;

2. it is necessary to relate and compare different constraints that are obtained at different spacetime positions. This often requires a spacetime dynamics and thus to specify a model as well as a cosmology;

3. it is necessary to relate the variations of different fundamental constants.

Therefore, we shall start in Section 2 by recalling the link between the constants of physics and the theories in which they appear, as well as with metrology. From a theoretical point of view, the constancy of the fundamental constants is deeply linked with the equivalence principle and general relativity. In Section 2 we will recall this relation and in particular the link with the universality of free fall. We will then summarize the various constraints that exist on such variations, mainly for the fine structure constant and for the gravitational constant in Sections 3 and 4, respectively. We will then turn to the theoretical implications in Section 5 in describing some of the arguments backing up the fact that constants are expected to vary, the main frameworks used in the literature and the various ways proposed to explain why they have the values we observe today. We shall finish by a discussion on their spatial variations in Section 6 and by discussing the possibility to understand their numerical values in Section 7.

Various reviews have been written on this topic. We will refer to the review [500] as FVC and we mention the following later reviews [31, 47, 72, 119, 226, 281, 278, 501, 395, 503, 505] and we refer to [356] for the numerical values of the constants adopted in this review. 


\section{Constants and Fundamental Physics}

\section{$2.1 \quad$ About constants}

Our physical theories introduce various structures to describe the phenomena of Nature. They involve various fields, symmetries and constants. These structures are postulated in order to construct a mathematically-consistent description of the known physical phenomena in the most unified and simple way.

We define the fundamental constants of a physical theory as any parameter that cannot be explained by this theory. Indeed, we are often dealing with other constants that in principle can be expressed in terms of these fundamental constants. The existence of these two sets of constants is important and arises from two different considerations. From a theoretical point of view we would like to extract the minimal set of fundamental constants, but often these constants are not measurable. From a more practical point of view, we need to measure constants, or combinations of constants, which allow us to reach the highest accuracy.

Therefore, these fundamental constants are contingent quantities that can only be measured. Such parameters have to be assumed constant in this theoretical framework for two reasons:

- from a theoretical point of view: the considered framework does not provide any way to compute these parameters, i.e., it does not have any equation of evolution for them since otherwise it would be considered as a dynamical field,

- from an experimental point of view: these parameters can only be measured. If the theories in which they appear have been validated experimentally, it means that, at the precisions of these experiments, these parameters have indeed been checked to be constant, as required by the necessity of the reproducibility of experimental results.

This means that testing for the constancy of these parameters is a test of the theories in which they appear and allow to extend our knowledge of their domain of validity. This also explains the definition chosen by Weinberg [526] who stated that they cannot be calculated in terms of other constants "... not just because the calculation is too complicated (as for the viscosity of water) but because we do not know of anything more fundamental".

This has a series of implications. First, the list of fundamental constants to consider depends on our theories of physics and, thus, on time. Indeed, when introducing new, more unified or more fundamental, theories the number of constants may change so that this list reflects both our knowledge of physics and, more important, our ignorance. Second, it also implies that some of these fundamental constants can become dynamical quantities in a more general theoretical framework so that the tests of the constancy of the fundamental constants are tests of fundamental physics, which can reveal that what was thought to be a fundamental constant is actually a field whose dynamics cannot be neglected. If such fundamental constants are actually dynamical fields it also means that the equations we are using are only approximations of other and more fundamental equations, in an adiabatic limit, and that an equation for the evolution of this new field has to be obtained.

The reflections on the nature of the constants and their role in physics are numerous. We refer to the books $[29,215,510,509]$ as well as [59, 165, 216, 393, 521, 526, 538] for various discussions of this issue that we cannot develop at length here. This paragraph summarizes some of the properties of the fundamental constants that have attracted some attention.

\subsubsection{Characterizing the fundamental constants}

Physical constants seem to play a central role in our physical theories since, in particular, they determined the magnitudes of the physical processes. Let us sketch briefly some of their properties. 
Table 1: List of the fundamental constants of our standard model. See Ref. [379] for further details on the measurements.

\begin{tabular}{lll}
\hline Constant & Symbol & Value \\
\hline Speed of light & $c$ & $299792458 \mathrm{~m} \mathrm{~s}^{-1}$ \\
Planck constant (reduced) & $\hbar$ & $1.054571628(53) \times 10^{-34} \mathrm{~J} \mathrm{~s}^{-2}$ \\
Newton constant & $G$ & $6.67428(67) \times 10^{-11} \mathrm{~m}^{2} \mathrm{~kg}^{-1} \mathrm{~s}^{-2}$ \\
\hline Weak coupling constant $\left(\right.$ at $\left.m_{Z}\right)$ & $g_{2}\left(m_{Z}\right)$ & $0.6520 \pm 0.0001$ \\
Strong coupling constant $\left(\right.$ at $\left.m_{Z}\right)$ & $g_{3}\left(m_{Z}\right)$ & $1.221 \pm 0.022$ \\
Weinberg angle & $\sin ^{2} \theta_{\mathrm{W}}(91.2 \mathrm{GeV})_{\overline{\mathrm{MS}}}$ & $0.23120 \pm 0.00015$ \\
\hline Electron Yukawa coupling & $h_{\mathrm{e}}$ & $2.94 \times 10^{-6}$ \\
Muon Yukawa coupling & $h_{\mu}$ & 0.000607 \\
Tauon Yukawa coupling & $h_{\tau}$ & 0.0102156 \\
Up Yukawa coupling & $h_{\mathrm{u}}$ & $0.000016 \pm 0.000007$ \\
Down Yukawa coupling & $h_{\mathrm{d}}$ & $0.00003 \pm 0.00002$ \\
Charm Yukawa coupling & $h_{\mathrm{c}}$ & $0.0072 \pm 0.0006$ \\
Strange Yukawa coupling & $h_{\mathrm{s}}$ & $0.0006 \pm 0.0002$ \\
Top Yukawa coupling & $h_{\mathrm{t}}$ & $1.002 \pm 0.029$ \\
Bottom Yukawa coupling & $h_{\mathrm{b}}$ & $0.026 \pm 0.003$ \\
\hline Quark CKM matrix angle & $\sin \theta_{12}$ & $0.2243 \pm 0.0016$ \\
& $\sin \theta_{23}$ & $0.0413 \pm 0.0015$ \\
Quark CKM matrix phase & $\sin \theta_{13}$ & $0.0037 \pm 0.0005$ \\
\hline Higgs potential quadratic coefficient & $\delta_{\mathrm{CKM}}$ & $1.05 \pm 0.24$ \\
Higgs potential quartic coefficient & $\hat{\mu}^{2}$ & $?$ \\
QCD vacuum phase & $\lambda$ & $?$ \\
\hline & $\theta_{\mathrm{QCD}}$ & $<10^{-9}$ \\
\hline
\end{tabular}

How many fundamental constants should be considered? The set of constants, which are conventionally considered as fundamental [213] consists of the electron charge $e$, the electron mass $m_{\mathrm{e}}$, the proton mass $m_{\mathrm{p}}$, the reduced Planck constant $\hbar$, the velocity of light in vacuum $c$, the Avogadro constant $N_{\mathrm{A}}$, the Boltzmann constant $k_{\mathrm{B}}$, the Newton constant $G$, the permeability and permittivity of space, $\varepsilon_{0}$ and $\mu_{0}$. The latter has a fixed value in the SI system of unit $\left(\mu_{0}=4 \pi \times 10^{-7} \mathrm{H} \mathrm{m}^{-1}\right)$, which is implicit in the definition of the Ampere; $\varepsilon_{0}$ is then fixed by the relation $\varepsilon_{0} \mu_{0}=c^{-2}$.

However, it is clear that this cannot correspond to the list of the fundamental constants, as defined earlier as the free parameters of the theoretical framework at hand. To define such a list we must specify this framework. Today, gravitation is described by general relativity, and the three other interactions and the matter fields are described by the standard model of particle physics. It follows that one has to consider 22 unknown constants (i.e., 19 unknown dimensionless parameters): the Newton constant $G, 6$ Yukawa couplings for the quarks $\left(h_{\mathrm{u}}, h_{\mathrm{d}}, h_{\mathrm{c}}, h_{\mathrm{s}}, h_{\mathrm{t}}, h_{\mathrm{b}}\right)$ and 3 for the leptons $\left(h_{\mathrm{e}}, h_{\mu}, h_{\tau}\right), 2$ parameters of the Higgs field potential $(\hat{\mu}, \lambda), 4$ parameters for the Cabibbo-Kobayashi-Maskawa matrix ( 3 angles $\theta_{i j}$ and a phase $\left.\delta_{\mathrm{CKM}}\right), 3$ coupling constants for the gauge groups $S U(3)_{c} \times S U(2)_{L} \times U(1)_{Y}\left(g_{1}, g_{2}, g_{3}\right.$ or equivalently $g_{2}, g_{3}$ and the Weinberg angle $\left.\theta_{\mathrm{W}}\right)$, and a phase for the QCD vacuum $\left(\theta_{\mathrm{QCD}}\right)$, to which one must add the speed of light $c$ and the Planck constant $h$. See Table 1 for a summary and their numerical values.

Again, this list of fundamental constants relies on what we accept as a fundamental theory. Today we have many hints that the standard model of particle physics has to be extended, in particular to include the existence of massive neutrinos. Such an extension will introduce at least seven new constants (3 Yukawa couplings and 4 Maki-Nakagawa-Sakata (MNS) parameters,

Living Reviews in Relativity

http: //www. livingreviews.org/lrr-2011-2 
similar to the CKM parameters). On the other hand, the number of constants can decrease if some unifications between various interaction exist (see Section 5.3.1 for more details) since the various coupling constants may be related to a unique coupling constant $\alpha_{U}$ and an energy scale of unification $M_{U}$ through

$$
\alpha_{i}^{-1}(E)=\alpha_{U}^{-1}+\frac{b_{i}}{2 \pi} \ln \frac{M_{U}}{E},
$$

where the $b_{i}$ are numbers, which depend on the explicit model of unification. Note that this would also imply that the variations, if any, of various constants shall be correlated.

Relation to other usual constants. These parameters of the standard model are related to various constants that will appear in this review (see Table 2). First, the quartic and quadratic coefficients of the Higgs field potential are related to the Higgs mass and vev, $m_{H}=\sqrt{-\hat{\mu}^{2} / 2}$ and $v=\sqrt{-\hat{\mu}^{2} / \lambda}$. The latter is related to the Fermi constant $G_{\mathrm{F}}=\left(v^{2} \sqrt{2}\right)^{-1}$, which imposes that $v=(246.7 \pm 0.2) \mathrm{GeV}$ while the Higgs mass is badly constrained. The masses of the quarks and leptons are related to their Yukawa coupling and the Higgs vev by $m=h v / \sqrt{2}$. The values of the gauge couplings depend on energy via the renormalization group so that they are given at a chosen energy scale, here the mass of the $Z$-boson, $m_{Z} \cdot g_{1}$ and $g_{2}$ are related by the Weinberg angle as $g_{1}=g_{2} \tan \theta_{\mathrm{W}}$. The electromagnetic coupling constant is not $g_{1}$ since $S U(2)_{L} \times U(1)_{Y}$ is broken to $U(1)_{\text {elec }}$ so that it is given by

$$
g_{\mathrm{EM}}\left(m_{Z}\right)=e=g_{2}\left(m_{Z}\right) \sin \theta_{\mathrm{W}} .
$$

Defining the fine-structure constant as $\alpha_{\mathrm{EM}}=g_{\mathrm{EM}}^{2} / \hbar c$, the (usual) zero energy electromagnetic fine structure constant is $\alpha_{\mathrm{EM}}=1 / 137.03599911(46)$ is related to $\alpha_{\mathrm{EM}}\left(m_{Z}\right)=1 /(127.918 \pm$ 0.018 ) by the renormalization group equations. In particular, it implies that $\alpha_{\mathrm{EM}} \sim \alpha\left(m_{Z}\right)+$ $\frac{2}{9 \pi} \ln \left(\frac{m_{Z}^{20}}{m_{\mathrm{u}}^{4} m_{\mathrm{c}}^{4} m_{\mathrm{d}} m_{\mathrm{s}} m_{\mathrm{b}} m_{\mathrm{e}}^{3} m_{\mu}^{3} m_{\tau}^{3}}\right)$. We define the QCD energy scale, $\Lambda_{\mathrm{QCD}}$, as the energy at which the strong coupling constant diverges. Note that it implies that $\Lambda_{\mathrm{QCD}}$ also depends on the Higgs and fermion masses through threshold effects.

More familiar constants, such as the masses of the proton and the neutron are, as we shall discuss in more detail below (see Section 5.3.2), more difficult to relate to the fundamental parameters because they depend not only on the masses of the quarks but also on the electromagnetic and strong binding energies.

Are some constants more fundamental? As pointed-out by Lévy-Leblond [328], all constants of physics do not play the same role, and some have a much deeper role than others. Following [328], we can define three classes of fundamental constants, class $A$ being the class of the constants characteristic of a particular system, class $B$ being the class of constants characteristic of a class of physical phenomena, and class $C$ being the class of universal constants. Indeed, the status of a constant can change with time. For instance, the velocity of light was initially a class A constant (describing a property of light), which then became a class B constant when it was realized that it was related to electromagnetic phenomena and, to finish, it ended as a type $\mathrm{C}$ constant (it enters special relativity and is related to the notion of causality, whatever the physical phenomena). It has even become a much more fundamental constant since it now enters in the definition of the meter [413] (see Ref. [510] for a more detailed discussion). This has to be contrasted with the proposition of Ref. [538] to distinguish the standard model free parameters as the gauge and gravitational couplings (which are associated to internal and spacetime curvatures) and the other parameters entering the accommodation of inertia in the Higgs sector.

Relation with physical laws. Lévy-Leblond [328] proposed to rank the constants in terms of their universality and he proposed that only three constants be considered to be of class C, 
Table 2: List of some related constants that appear in our discussions. See Ref. [379].

\begin{tabular}{lll}
\hline Constant & Symbol & Value \\
\hline Electromagnetic coupling constant & $g_{\mathrm{EM}}=e=g_{2} \sin \theta_{\mathrm{W}}$ & $0.313429 \pm 0.000022$ \\
Higgs mass & $m_{H}$ & $>100 \mathrm{GeV}$ \\
Higgs vev & $v$ & $(246.7 \pm 0.2) \mathrm{GeV}$ \\
Fermi constant & $G_{\mathrm{F}}=1 / \sqrt{2} v^{2}$ & $1.16637(1) \times 10^{-5} \mathrm{GeV}^{-2}$ \\
Mass of the $W^{ \pm}$ & $m_{W}$ & $80.398 \pm 0.025 \mathrm{GeV}$ \\
Mass of the $Z$ & $m_{Z}$ & $91.1876 \pm 0.0021 \mathrm{GeV}$ \\
Fine structure constant & $\alpha_{\mathrm{EM}}$ & $1 / 137.035999679(94)$ \\
Fine structure constant at $m_{Z}$ & $\alpha_{\mathrm{EM}}\left(m_{Z}\right)$ & $1 /(127.918 \pm 0.018)$ \\
Weak structure constant at $m_{Z}$ & $\alpha_{\mathrm{W}}\left(m_{Z}\right)$ & $0.03383 \pm 0.00001$ \\
Strong structure constant at $m_{Z}$ & $\alpha_{\mathrm{S}}\left(m_{Z}\right)$ & $0.1184 \pm 0.0007$ \\
Gravitational structure constant & $\alpha_{\mathrm{G}}=G m_{\mathrm{p}}^{2} / \hbar c$ & $\sim 5.905 \times 10^{-39}$ \\
Electron mass & $m_{\mathrm{e}}=h_{\mathrm{e}} v / \sqrt{2}$ & $510.998910 \pm 0.000013 \mathrm{keV}$ \\
Mu mass & $m_{\mu}=h_{\mu} v / \sqrt{2}$ & $105.658367 \pm 0.000004 \mathrm{MeV}$ \\
Tau mass & $m_{\tau}=h_{\tau} v / \sqrt{2}$ & $1776.84 \pm 0.17 \mathrm{MeV}$ \\
Up quark mass & $m_{\mathrm{u}}=h_{\mathrm{u}} v / \sqrt{2}$ & $(1.5-3.3) \mathrm{MeV}$ \\
Down quark mass & $m_{\mathrm{d}}=h_{\mathrm{d}} v / \sqrt{2}$ & $(3.5-6.0) \mathrm{MeV}$ \\
Strange quark mass & $m_{\mathrm{s}}=h_{\mathrm{s}} v / \sqrt{2}$ & $105_{-35}^{+25} \mathrm{MeV}$ \\
Charm quark mass & $m_{\mathrm{c}}=h_{\mathrm{c}} v / \sqrt{2}$ & $1.27_{-0.11}^{+0.07} \mathrm{GeV}$ \\
Bottom quark mass & $m_{\mathrm{b}}=h_{\mathrm{b}} v / \sqrt{2}$ & $4.20_{-0.07}^{+0.17} \mathrm{GeV}$ \\
Top quark mass & $m_{\mathrm{t}}=h_{\mathrm{t}} v / \sqrt{2}$ & $171.3 \pm 2.3 \mathrm{GeV}$ \\
QCD energy scale & $\Lambda_{\mathrm{QCD}}$ & $(190-240) \mathrm{MeV}$ \\
Mass of the proton & $m_{\mathrm{p}}$ & $938.272013 \pm 0.000023 \mathrm{MeV}$ \\
Mass of the neutron & $m_{\mathrm{n}}$ & $939.565346 \pm 0.000023 \mathrm{MeV}$ \\
proton-neutron mass difference & $Q_{\mathrm{np}}$ & $1.2933321 \pm 0.0000004 \mathrm{MeV}$ \\
proton-to-electron mass ratio & $\mu=m_{\mathrm{p}} / m_{\mathrm{e}}$ & 1836.15 \\
electron-to-proton mass ratio & $\bar{\mu}=m_{\mathrm{e}} / m_{\mathrm{p}}$ & $1 / 1836.15$ \\
$d-u$ quark mean mass & $m_{\mathrm{q}}=\left(m_{\mathrm{u}}+m_{\mathrm{d}}\right) / 2$ & $(2.5-5.0) \mathrm{MeV}$ \\
$d-u$ quark mass difference & $\delta m_{\mathrm{q}}=m_{\mathrm{d}}-m_{\mathrm{u}}$ & $(0.2-4.5) \mathrm{MeV}$ \\
proton gyromagnetic factor & $g_{\mathrm{p}}$ & 5.586 \\
neutron gyromagnetic factor & $g_{\mathrm{n}}$ & -3.826 \\
Rydberg constant & $R_{\infty}$ & $10973731.568527(73) \mathrm{m}^{-1}$ \\
\hline & & \\
& & \\
\hline
\end{tabular}

namely $G, \hbar$ and $c$. He pointed out two important roles of these constants in the laws of physics. First, they act as concept synthesizer during the process of our understanding of the laws of nature: contradictions between existing theories have often been resolved by introducing new concepts that are more general or more synthetic than older ones. Constants build bridges between quantities that were thought to be incommensurable and thus allow new concepts to emerge. For example $c$ underpins the synthesis of space and time while the Planck constant allowed to related the concept of energy and frequency and the gravitational constant creates a link between matter and spacetime. Second, it follows that these constants are related to the domains of validity of these theories. For instance, as soon as velocity approaches $c$, relativistic effects become important, relativistic effects cannot be negligible. On the other hand, for speed much below $c$, Galilean kinematics is sufficient. Planck constant also acts as a referent, since if the action of a system greatly exceeds the value of that constant, classical mechanics will be appropriate to describe this system. While the place of $c$ (related to the notion of causality) and $\hbar$ (related to the quantum) in this list are well argued, the place of $G$ remains debated since it is thought that it will have to be replaced by some mass scale. 
Evolution. There are many ways the list of constants can change with our understanding of physics. First, new constants may appear when new systems or new physical laws are discovered; this is, for instance, the case of the charge of the electron or more recently the gauge couplings of the nuclear interactions. A constant can also move from one class to a more universal class. An example is that of the electric charge, initially of class A (characteristic of the electron), which then became class B when it was understood that it characterizes the strength of the electromagnetic interaction. A constant can also disappear from the list, because it is either replaced by more fundamental constants (e.g., the Earth acceleration due to gravity and the proportionality constant entering Kepler law both disappeared because they were "explained" in terms of the Newton constant and the mass of the Earth or the Sun) or because it can happen that a better understanding of physics teaches us that two hitherto distinct quantities have to be considered as a single phenomenon (e.g., the understanding by Joule that heat and work were two forms of energy led to the fact that the Joule constant, expressing the proportionality between work and heat, lost any physical meaning and became a simple conversion factor between units used in the measurement of heat (calories) and work (Joule)). Nowadays the calorie has fallen in disuse. Indeed demonstrating that a constant is varying will have direct implications on our list of constants.

In conclusion, the evolution of the number, status of the constants can teach us a lot about the evolution of the ideas and theories in physics since it reflects the birth of new concepts, their evolution and unification with other ones.

\subsubsection{Constants and metrology}

Since we cannot compute them in the theoretical framework in which they appear, it is a crucial property of the fundamental constants (but in fact of all the constants) that their value can be measured. The relation between constants and metrology is a huge subject to which we just draw the attention on some selected aspects. For more discussions, see [56, 280, 278].

The introduction of constants in physical laws is also closely related to the existence of systems of units. For instance, Newton's law states that the gravitational force between two masses is proportional to each mass and inversely proportional to the square of their separation. To transform the proportionality to an equality one requires the use of a quantity with dimension of $\mathrm{m}^{3} \mathrm{~kg}^{-1} \mathrm{~s}^{-2}$ independent of the separation between the two bodies, of their mass, of their composition (equivalence principle) and on the position (local position invariance). With an other system of units the numerical value of this constant could have simply been anything. Indeed, the numerical value of any constant crucially depends on the definition of the system of units.

Measuring constants. The determination of the laboratory value of constants relies mainly on the measurements of lengths, frequencies, times, ... (see [414] for a treatise on the measurement of constants and [213] for a recent review). Hence, any question on the variation of constants is linked to the definition of the system of units and to the theory of measurement. The behavior of atomic matter is determined by the value of many constants. As a consequence, if, e.g., the fine-structure constant is spacetime dependent, the comparison between several devices such as clocks and rulers will also be spacetime dependent. This dependence will also differ from one clock to another so that metrology becomes both device and spacetime dependent, a property that will actually be used to construct tests of the constancy of the constants.

Indeed a measurement is always a comparison between two physical systems of the same dimensions. This is thus a relative measurement, which will give as result a pure number. This trivial statement is oversimplifying since in order to compare two similar quantities measured separately, one needs to perform a number of comparisons. In order to reduce the number of comparisons (and in particular to avoid creating every time a chain of comparisons), a certain set of them has been included in the definitions of units. Each units can then be seen as an abstract physical sys- 
tem, which has to be realized effectively in the laboratory, and to which another physical system is compared. A measurement in terms of these units is usually called an absolute measurement. Most fundamental constants are related to microscopic physics and their numerical values can be obtained either from a pure microscopic comparison (as is, e.g., the case for $m_{\mathrm{e}} / m_{\mathrm{p}}$ ) or from a comparison between microscopic and macroscopic values (for instance to deduce the value of the mass of the electron in kilogram). This shows that the choice of the units has an impact on the accuracy of the measurement since the pure microscopic comparisons are in general more accurate than those involving macroscopic physics. This implies that only the variation of dimensionless constants can be measured and in case such a variation is detected, it is impossible to determine, which dimensional constant is varying [183].

It is also important to stress that in order to deduce the value of constants from an experiment, one usually needs to use theories and models. An example [278] is provided by the Rydberg constant. It can easily be expressed in terms of some fundamental constants as $R_{\infty}=\alpha_{\mathrm{EM}}^{2} m_{\mathrm{e}} c / 2 h$. It can be measured from, e.g., the triplet $1 s-2 s$ transition in hydrogen, the frequency of which is related to the Rydberg constant and other constants by assuming QED so that the accuracy of $R_{\infty}$ is much lower than that of the measurement of the transition. This could be solved by defining $R_{\infty}$ as $4 \nu_{\mathrm{H}}(1 s-2 s) / 3 c$ but then the relation with more fundamental constants would be more complicated and actually not exactly known. This illustrates the relation between a practical and a fundamental approach and the limitation arising from the fact that we often cannot both exactly calculate and directly measure some quantity. Note also that some theoretical properties are plugged in the determination of the constants.

As a conclusion, let us recall that (i) in general, the values of the constants are not determined by a direct measurement but by a chain involving both theoretical and experimental steps, (ii) they depend on our theoretical understanding, (iii) the determination of a self-consistent set of values of the fundamental constants results from an adjustment to achieve the best match between theory and a defined set of experiments (which is important because we actually know that the theories are only good approximation and have a domain of validity) (iv) that the system of units plays a crucial role in the measurement chain, since for instance in atomic units, the mass of the electron could have been obtained directly from a mass ratio measurement (even more precise!) and (v) fortunately the test of the variability of the constants does not require a priori to have a high-precision value of the considered constants.

System of units. Thus, one needs to define a coherent system of units. This has a long, complex and interesting history that was driven by simplicity and universality but also by increasing stability and accuracy [29, 509].

Originally, the sizes of the human body were mostly used to measure the length of objects (e.g., the foot and the thumb gave feet and inches) and some of these units can seem surprising to us nowadays (e.g., the span was the measure of hand with fingers fully splayed, from the tip of the thumb to the tip of the little finger!). Similarly weights were related to what could be carried in the hand: the pound, the ounce, the dram... Needless to say, this system had a few disadvantages since each country, region has its own system (for instance in France there was more than 800 different units in use in 1789). The need to define a system of units based on natural standard led to several propositions to define a standard of length (e.g., the mille by Gabriel Mouton in 1670 defined as the length of one angular minute of a great circle on the Earth or the length of the pendulum that oscillates once a second by Jean Picard and Christiaan Huygens). The real change happened during the French Revolution during which the idea of a universal and non anthropocentric system of units arose. In particular, the Assemblée adopted the principle of a uniform system of weights and measures on 8 May 1790 and, in March 1791 a decree (these texts are reprinted in [510]) was voted, stating that a quarter of the terrestrial meridian would be the basis of the definition of the meter (from the Greek metron, as proposed by Borda): a meter would

Living Reviews in Relativity

http: //www. livingreviews.org/lrr-2011-2 
henceforth be one ten millionth part of a quarter of the terrestrial meridian. Similarly the gram was defined as the mass of one cubic centimeter of distilled water (at a precise temperature and pressure) and the second was defined from the property that a mean solar day must last 24 hours.

To make a long story short, this led to the creation of the metric system and then of the signature of La convention du mètre in 1875. Since then, the definition of the units have evolved significantly. First, the definition of the meter was related to more immutable systems than our planet, which, as pointed out by Maxwell in 1870, was an arbitrary and inconstant reference. He then suggested that atoms may be such a universal reference. In 1960, the International Bureau of Weights and Measures (BIPM) established a new definition of the meter as the length equal to 1650763 wavelengths, in a vacuum, of the transition line between the levels $2 p_{10}$ and $5 d_{5}$ of krypton-86. Similarly the rotation of the Earth was not so stable and it was proposed in 1927 by André Danjon to use the tropical year as a reference, as adopted in 1952. In 1967, the second was also related to an atomic transition, defined as the duration of 9162631770 periods of the transition between the two hyperfine levels of the ground state of caesium-133. To finish, it was decided in 1983, that the meter shall be defined by fixing the value of the speed of light to $c=299792458 \mathrm{~m} \mathrm{~s}^{-1}$ and we refer to [55] for an up to date description of the SI system. Today, the possibility to redefine the kilogram in terms of a fixed value of the Planck constant is under investigation [279].

This summary illustrates that the system of units is a human product and all SI definitions are historically based on non-relativistic classical physics. The changes in the definition were driven by the will to use more stable and more fundamental quantities so that they closely follow the progress of physics. This system has been created for legal use and indeed the choice of units is not restricted to SI.

SI systems and the number of basic units. The International System of Units defines seven basic units: the meter $(\mathrm{m})$, second $(\mathrm{s})$ and kilogram $(\mathrm{kg})$, the Ampere $(\mathrm{A})$, Kelvin $(\mathrm{K})$, mole (mol) and candela (cd), from which one defines secondary units. While needed for pragmatic reasons, this system of units is unnecessarily complicated from the point of view of theoretical physics. In particular, the Kelvin, mole and candela are derived from the four other units since temperature is actually a measure of energy, the candela is expressed in terms of energy flux so that both can be expressed in mechanical units of length $[\mathrm{L}]$, mass $[\mathrm{M}]$ and time $[\mathrm{T}]$. The mole is merely a unit denoting numbers of particles and has no dimension.

The status of the Ampere is interesting in itself. The discovery of the electric charge $[\mathrm{Q}]$ led to the introduction of a new units, the Coulomb. The Coulomb law describes the force between two charges as being proportional to the product of the two charges and to the inverse of the distance squared. The dimension of the force being known as $\left[\mathrm{MLT}^{-2}\right]$, this requires the introduction of a new constant $\varepsilon_{0}$ (which is only a conversion factor), with dimensions $\left[\mathrm{Q}^{2} \mathrm{M}^{-1} \mathrm{~L}^{-3} \mathrm{~T}^{2}\right]$ in the Coulomb law, and that needs to be measured. Another route could have been followed since the Coulomb law tells us that no new constant is actually needed if one uses $\left[\mathrm{M}^{1 / 2} \mathrm{~L}^{3 / 2} \mathrm{~T}^{-1}\right]$ as the dimension of the charge. In this system of units, known as Gaussian units, the numerical value of $\varepsilon_{0}$ is 1 . Hence the Coulomb can be expressed in terms of the mechanical units $[\mathrm{L}],[\mathrm{M}]$ and $[\mathrm{T}]$, and so will the Ampere. This reduces the number of conversion factors, that need to be experimentally determined, but note that both choices of units assume the validity of the Coulomb law.

Natural units. The previous discussion tends to show that all units can be expressed in terms of the three mechanical units. It follows, as realized by Johnstone Stoney in $1874^{2}$, that these three basic units can be defined in terms of 3 independent constants. He proposed [27, 267] to

\footnotetext{
2 After studying electrolysis in 1874, Johnstone Stoney suggested the existence of a "single definite quantity of electricity". He was able to estimate the value of this elementary charge by means of Faraday's laws of electrolysis. He introduced the term "electron" in 1894 and it was identified as a particle in 1897 by Thomson.
} 
use three constants: the Newton constant, the velocity of light and the basic units of electricity, i.e., the electron charge, in order to define, from dimensional analysis a "natural series of physical units" defined as

$$
\begin{aligned}
& t_{\mathrm{S}}=\sqrt{\frac{G e^{2}}{4 \pi \varepsilon_{0} c^{6}}} \sim 4.59 \times 10^{-45} \mathrm{~s}, \\
& \ell_{\mathrm{S}}=\sqrt{\frac{G e^{2}}{4 \pi \varepsilon_{0} c^{4}}} \sim 1.37 \times 10^{-36} \mathrm{~m} \\
& m_{\mathrm{S}}=\sqrt{\frac{e^{2}}{4 \pi \varepsilon_{0} G}} \sim 1.85 \times 10^{-9} \mathrm{~kg}
\end{aligned}
$$

where the $\varepsilon_{0}$ factor has been included because we are using the SI definition of the electric charge. In such a system of units, by construction, the numerical value of $G, e$ and $c$ is 1 , i.e., $c=1 \times \ell_{\mathrm{S}} \cdot t_{\mathrm{S}}^{-1}$ etc.

A similar approach to the definition of the units was independently proposed by Planck [418] on the basis of the two constants $a$ and $b$ entering the Wien law and $G$, which he reformulated later [419] in terms of $c, G$ and $\hbar$ as

$$
\begin{aligned}
t_{\mathrm{P}} & =\sqrt{\frac{G \hbar}{c^{5}}} \sim 5.39056 \times 10^{-44} \mathrm{~s}, \\
\ell_{\mathrm{P}} & =\sqrt{\frac{G \hbar}{c^{3}}} \sim 1.61605 \times 10^{-35} \mathrm{~m}, \\
m_{\mathrm{P}} & =\sqrt{\frac{\hbar c}{G}} \sim 2.17671 \times 10^{-8} \mathrm{~kg} .
\end{aligned}
$$

The two systems are clearly related by the fine-structure constant since $e^{2} / 4 \pi \varepsilon_{0}=\alpha_{\mathrm{EM}} h c$.

Indeed, we can construct many such systems since the choice of the 3 constants is arbitrary. For instance, we can construct a system based on $\left(e, m_{\mathrm{e}}, h\right)$, that we can call the Bohr units, which will be suited to the study of the atom. The choice may be dictated by the system, which is studied (it is indeed far fetched to introduce $G$ in the construction of the units when studying atomic physics) so that the system is well adjusted in the sense that the numerical values of the computations are expected to be of order unity in these units.

Such constructions are very useful for theoretical computations but not adapted to measurement so that one needs to switch back to SI units. More important, this shows that, from a theoretical point of view, one can define the system of units from the laws of nature, which are supposed to be universal and immutable.

Do we actually need 3 natural units? is an issue debated at length. For instance, Duff, Okun and Veneziano [165] respectively argue for none, three and two (see also [535]). Arguing for no fundamental constant leads to consider them simply as conversion parameters. Some of them are, like the Boltzmann constant, but some others play a deeper role in the sense that when a physical quantity becomes of the same order as this constant, new phenomena appear; this is the case, e.g., of $\hbar$ and $c$, which are associated respectively to quantum and relativistic effects. Okun [392] considered that only three fundamental constants are necessary, as indicated by the International System of Units. In the framework of quantum field theory + general relativity, it seems that this set of three constants has to be considered and it allows to classify the physical theories (with the famous cube of physical theories). However, Veneziano [514] argued that in the framework of string theory one requires only two dimensionful fundamental constants, $c$ and the

Living Reviews in Relativity

http://www. livingreviews . org//rr-2011-2 
string length $\lambda_{s}$. The use of $\hbar$ seems unnecessary since it combines with the string tension to give $\lambda_{s}$. In the case of the Nambu-Goto action $S / \hbar=(T / \hbar) \int \mathrm{d}($ Area $) \equiv \lambda_{s}^{-2} \int \mathrm{d}($ Area $)$ and the Planck constant is just given by $\lambda_{s}^{-2}$. In this view, $\hbar$ has not disappeared but has been promoted to the role of a UV cut-off that removes both the infinities of quantum field theory and singularities of general relativity. This situation is analogous to pure quantum gravity [388] where $\hbar$ and $G$ never appear separately but only in the combination $\ell_{\mathrm{Pl}}=\sqrt{G \hbar / c^{3}}$ so that only $c$ and $\ell_{\mathrm{Pl}}$ are needed. Volovik [520] made an analogy with quantum liquids to clarify this. There an observer knows both the effective and microscopic physics so that he can judge whether the fundamental constants of the effective theory remain fundamental constants of the microscopic theory. The status of a constant depends on the considered theory (effective or microscopic) and, more interestingly, on the observer measuring them, i.e., on whether this observer belongs to the world of low-energy quasi-particles or to the microscopic world.

Fundamental parameters. Once a set of three independent constants has been chosen as natural units, then all other constants are dimensionless quantities. The values of these combinations of constants does not depend on the way they are measured, [110, 164, 437], on the definition of the units etc.... It follows that any variation of constants that will leave these numbers unaffected is actually just a redefinition of units.

These dimensionless numbers represent, e.g., the mass ratio, relative magnitude of strength etc.... Changing their values will indeed have an impact on the intensity of various physical phenomena, so that they encode some properties of our world. They have specific values (e.g., $\alpha_{\mathrm{EM}} \sim 1 / 137, m_{\mathrm{p}} / m_{\mathrm{e}} \sim 1836$, etc.) that we may hope to understand. Are all these numbers completely contingent, or are some (why not all?) of them related by relations arising from some yet unknown and more fundamental theories. In such theories, some of these parameters may actually be dynamical quantities and, thus, vary in space and time. These are our potential varying constants.

\subsection{The constancy of constants as a test of general relativity}

The previous paragraphs have yet emphasize why testing for the consistency of the constants is a test of fundamental physics since it can reveal the need for new physical degrees of freedom in our theory. We now want to stress the relation of this test with other tests of general relativity and with cosmology.

\subsubsection{General relativity}

The tests of the constancy of fundamental constants take all their importance in the realm of the tests of the equivalence principle [540]. Einstein general relativity is based on two independent hypotheses, which can conveniently be described by decomposing the action of the theory as $S=S_{\text {grav }}+S_{\text {matter }}$.

The equivalence principle has strong implication for the functional form of $S_{\mathrm{grav}}$. This principle includes three hypotheses:

- the universality of free fall,

- the local position invariance,

- the local Lorentz invariance.

In its weak form (that is for all interactions but gravity), it is satisfied by any metric theory of gravity and general relativity is conjectured to satisfy it in its strong form (that is for all interactions including gravity). We refer to [540] for a detailed description of these principles. The 
weak equivalence principle can be mathematically implemented by assuming that all matter fields are minimally coupled to a single metric tensor $g_{\mu \nu}$. This metric defines the length and times measured by laboratory clocks and rods so that it can be called the physical metric. This implies that the action for any matter field, $\psi$ say, can be written as

$$
S_{\text {matter }}\left(\psi, g_{\mu \nu}\right) \text {. }
$$

This metric coupling ensures in particular the validity of the universality of free-fall. Since locally, in the neighborhood of the worldline, there always exists a change of coordinates so that the metric takes a Minkowskian form at lowest order, the gravitational field can be locally "effaced" (up to tidal effects). If we identify this neighborhood to a small lab, this means that any physical properties that can be measured in this lab must be independent of where and when the experiments are carried out. This is indeed the assumption of local position invariance, which implies that the constants must take the same value independent of the spacetime point where they are measured. Thus, testing the constancy of fundamental constants is a direct test of this principle and therefore of the metric coupling. Interestingly, the tests we are discussing in this review allow one to extend them much further than the solar scales and even in the early universe, an important information to check the validity of relativity in cosmology.

As an example, the action of a point-particle reads

$$
S_{\text {matter }}=-\int m c \sqrt{-g_{\mu \nu}(\mathbf{x}) v^{\mu} v^{\nu}} \mathrm{d} t
$$

with $v^{\mu} \equiv \mathrm{d} x^{\mu} / \mathrm{d} t$. The equation of motion that one derives from this action is the usual geodesic equation

$$
a^{\mu} \equiv u^{\nu} \nabla_{\nu} u^{\mu}=0 \text {, }
$$

where $u^{\mu}=\mathrm{d} x^{\mu} / c \mathrm{~d} \tau, \tau$ being the proper time; $\nabla_{\mu}$ is the covariant derivative associated with the metric $g_{\mu \nu}$ and $a^{\nu}$ is the 4-acceleration. Any metric theory of gravity will enjoy such a matter Lagrangian and the worldline of any test particle shall be a geodesic of the spacetime with metric $g_{\mu \nu}$, as long as there is no other long range force acting on it (see [190] for a detailed review of motion in alternative theories of gravity).

Note that in the Newtonian limit $g_{00}=-1-2 \Phi_{N} / c^{2}$ where $\Phi_{N}$ is the Newtonian potential. It follows that, in the slow velocity limit, the geodesic equation reduces to

$$
\dot{\mathbf{v}}=\mathbf{a}=-\nabla \Phi_{N} \equiv \mathbf{g}_{N}
$$

hence defining the Newtonian acceleration $\mathbf{g}_{N}$. Recall that the proper time of a clock is related to the coordinate time by $\mathrm{d} \tau=\sqrt{-g_{00}} \mathrm{~d} t$. Thus, if one exchanges electromagnetic signals between two identical clocks in a stationary situation, the apparent difference between the two clocks rates will be

$$
\frac{\nu_{1}}{\nu_{2}}=1+\frac{\Phi_{N}(2)-\Phi_{N}(1)}{c^{2}}
$$

at lowest order. This is the universality of gravitational redshift.

The assumption of a metric coupling is actually well tested in the solar system:

- First, it implies that all non-gravitational constants are spacetime independent, which have been tested to a very high accuracy in many physical systems and for various fundamental constants; this is the subject of this review.

- Second, the isotropy has been tested from the constraint on the possible quadrupolar shift of nuclear energy levels [99, 304, 422] proving that different matter fields couple to a unique metric tensor at the $10^{-27}$ level. 
Table 3: Summary of the constraints on the violation of the universality of free fall.

\begin{tabular}{lccc}
\hline Constraint & Body 1 & Body 2 & Ref. \\
\hline$(-1.9 \pm 2.5) \times 10^{-12}$ & $\mathrm{Be}$ & $\mathrm{Cu}$ & {$[4]$} \\
$(0.1 \pm 2.7 \pm 1.7) \times 10^{-13}$ & Earth-like rock & Moon-like rock & {$[23]$} \\
$(-1.0 \pm 1.4) \times 10^{-13}$ & Earth & Moon & {$[543]$} \\
$(0.3 \pm 1.8) \times 10^{-13}$ & $\mathrm{Te}$ & $\mathrm{Bi}$ & {$[450]$} \\
$(-0.2 \pm 2.8) \times 10^{-12}$ & $\mathrm{Be}$ & $\mathrm{Al}$ & {$[481]$} \\
$(-1.9 \pm 2.5) \times 10^{-12}$ & $\mathrm{Be}$ & $\mathrm{Cu}$ & {$[481]$} \\
$(5.1 \pm 6.7) \times 10^{-12}$ & $\mathrm{Si} / \mathrm{Al}$ & $\mathrm{Cu}$ & {$[481]$} \\
\hline
\end{tabular}

- Third, the universality of free fall can be tested by comparing the accelerations of two test bodies in an external gravitational field. The parameter $\eta_{12}$ defined as

$$
\eta_{12} \equiv 2 \frac{\left|\mathbf{a}_{1}-\mathbf{a}_{2}\right|}{\left|\mathbf{a}_{1}+\mathbf{a}_{2}\right|},
$$

can be constrained experimentally, e.g., in the laboratory by comparing the acceleration of a beryllium and a copper mass in the Earth gravitational field [4] to get

$$
\eta_{\mathrm{Be}, \mathrm{Cu}}=(-1.9 \pm 2.5) \times 10^{-12} .
$$

Similarly the comparison of Earth-core-like and Moon-mantle-like bodies gave [23]

$$
\eta_{\text {Earth,Moon }}=(0.1 \pm 2.7 \pm 1.7) \times 10^{-13},
$$

and experiments with torsion balance using test bodies composed of tellurium an bismuth allowed to set the constraint [450]

$$
\eta_{\mathrm{Te}, \mathrm{Bi}}=(0.3 \pm 1.8) \times 10^{-13} .
$$

The Lunar Laser ranging experiment [543], which compares the relative acceleration of the Earth and Moon in the gravitational field of the Sun, also set the constraints

$$
\eta_{\text {Earth,Moon }}=(-1.0 \pm 1.4) \times 10^{-13} .
$$

Note that since the core represents only $1 / 3$ of the mass of the Earth, and since the Earth's mantle has the same composition as that of the Moon (and thus shall fall in the same way), one loses a factor of three, so that this constraint is actually similar to the one obtained in the lab. Further constraints are summarized in Table 3. The latter constraint also contains some contribution from the gravitational binding energy and thus includes the strong equivalence principle. When the laboratory result of [23] is combined with the LLR results of [542] and [365], one gets a constraints on the strong equivalence principle parameter, respectively

$$
\eta_{\mathrm{SEP}}=(3 \pm 6) \times 10^{-13} \text { and } \eta_{\mathrm{SEP}}=(-4 \pm 5) \times 10^{-13} .
$$

Large improvements are expected thanks to existence of two dedicated space mission projects: Microscope [493] and STEP [355].

- Fourth, the Einstein effect (or gravitational redshift) has been measured at the $2 \times 10^{-4}$ level [517]. 
We can conclude that the hypothesis of metric coupling is extremely well-tested in the solar system.

The second building block of general relativity is related to the dynamics of the gravitational sector, assumed to be dictated by the Einstein-Hilbert action

$$
S_{\text {grav }}=\frac{c^{3}}{16 \pi G} \int \sqrt{-g_{*}} R_{*} \mathrm{~d}^{4} x .
$$

This defines the dynamics of a massless spin-2 field $g_{\mu \nu}^{*}$, called the Einstein metric. General relativity then assumes that both metrics coincide, $g_{\mu \nu}=g_{\mu \nu}^{*}$ (which is related to the strong equivalence principle), but it is possible to design theories in which this is indeed not the case (see the example of scalar-tensor theories below; Section 5.1.1) so that general relativity is one out of a large family of metric theories.

The variation of the total action with respect to the metric yields the Einstein equations

$$
R_{\mu \nu}-\frac{1}{2} R g_{\mu \nu}=\frac{8 \pi G}{c^{4}} T_{\mu \nu}
$$

where $T^{\mu \nu} \equiv(2 / \sqrt{-g}) \delta S_{\text {matter }} / \delta g_{\mu \nu}$ is the matter stress-energy tensor. The coefficient $8 \pi G / c^{4}$ is determined by the weak-field limit of the theory that should reproduce the Newtonian predictions.

The dynamics of general relativity can be tested in the solar system by using the parameterized post-Newtonian formalism (PPN). Its is a general formalism that introduces 10 phenomenological parameters to describe any possible deviation from general relativity at the first post-Newtonian order [540, 541] (see also [60] for a review on higher orders). The formalism assumes that gravity is described by a metric and that it does not involve any characteristic scale. In its simplest form, it reduces to the two Eddington parameters entering the metric of the Schwartzschild metric in isotropic coordinates

$$
g_{00}=-1+\frac{2 G m}{r c^{2}}-2 \beta^{\mathrm{PPN}}\left(\frac{2 G m}{r c^{2}}\right)^{2}, \quad g_{i j}=\left(1+2 \gamma^{\mathrm{PPN}} \frac{2 G m}{r c^{2}}\right) \delta_{i j} .
$$

Indeed, general relativity predicts $\beta^{\mathrm{PPN}}=\gamma^{\mathrm{PPN}}=1$.

These two phenomenological parameters are constrained (1) by the shift of the Mercury perihelion [457], which implies that $\left|2 \gamma^{\mathrm{PPN}}-\beta^{\mathrm{PPN}}-1\right|<3 \times 10^{-3}$, (2) the Lunar laser ranging experiments [543], which implies that $\left|4 \beta^{\mathrm{PPN}}-\gamma^{\mathrm{PPN}}-3\right|=(4.4 \pm 4.5) \times 10^{-4}$ and $(3)$ by the deflection of electromagnetic signals, which are all controlled by $\gamma^{\mathrm{PPN}}$. For instance the very long baseline interferometry [459] implies that $\left|\gamma^{\mathrm{PPN}}-1\right|=4 \times 10^{-4}$, while the measurement of the time delay variation to the Cassini spacecraft [53] sets $\gamma^{\mathrm{PPN}}-1=(2.1 \pm 2.3) \times 10^{-5}$.

The PPN formalism does not allow to test finite range effects that could be caused, e.g., by a massive degree of freedom. In that case one expects a Yukawa-type deviation from the Newton potential,

$$
V=\frac{G m}{r}\left(1+\alpha \mathrm{e}^{-r / \lambda}\right)
$$

that can be probed by "fifth force" experimental searches. $\lambda$ characterizes the range of the Yukawa deviation of strength $\alpha$. The constraints on $(\lambda, \alpha)$ are summarized in [256], which typically shows that $\alpha<10^{-2}$ on scales ranging from the millimeter to the solar system size.

General relativity is also tested with pulsars [125, 189] and in the strong field regime [425]. For more details we refer to $[129,495,540,541]$. Needless to say that any extension of general relativity has to pass these constraints. However, deviations from general relativity can be larger in the past, as we shall see, which makes cosmology an interesting physical system to extend these constraints. 


\subsubsection{Varying constants and the universality of free fall}

As the previous description shows, the constancy of the fundamental constants and the universality are two pillars of the equivalence principle. Dicke [152] realized that they are actually not independent and that if the coupling constants are spatially dependent then this will induce a violation of the universality of free fall.

The connection lies in the fact that the mass of any composite body, starting, e.g., from nuclei, includes the mass of the elementary particles that constitute it (this means that it will depend on the Yukawa couplings and on the Higgs sector parameters) but also a contribution, $E_{\text {binding }} / c^{2}$, arising from the binding energies of the different interactions (i.e., strong, weak and electromagnetic) but also gravitational for massive bodies. Thus, the mass of any body is a complicated function of all the constants, $m\left[\alpha_{i}\right]$.

It follows that the action for a point particle is no more given by Equation (2) but by

$$
S_{\text {matter }}=-\int m_{A}\left[\alpha_{j}\right] c \sqrt{-g_{\mu \nu}(\mathbf{x}) v^{\mu} v^{\nu}} \mathrm{d} t,
$$

where $\alpha_{j}$ is a list of constants including $\alpha_{\mathrm{EM}}$ but also many others and where the index $A$ in $m_{A}$ recalls that the dependency in these constants is a priori different for bodies of different chemical composition. The variation of this action gives the equation of motion

$$
u^{\nu} \nabla_{\nu} u^{\mu}=-\left(\sum_{i} \frac{\partial \ln m_{A}}{\partial \alpha_{i}} \frac{\partial \alpha_{i}}{\partial x^{\beta}}\right)\left(g^{\beta \mu}+u^{\beta} u^{\mu}\right) .
$$

It follows that a test body will not enjoy a geodesic motion. In the Newtonian limit $g_{00}=$ $-1+2 \Phi_{N} / c^{2}$, and at first order in $v / c$, the equation of motion of a test particle reduces to

$$
\mathbf{a}=\mathbf{g}_{N}+\delta \mathbf{a}_{A}, \quad \delta \mathbf{a}_{A}=-c^{2} \sum_{i} f_{A, i}\left(\nabla \alpha_{i}+\dot{\alpha}_{i} \frac{\mathbf{v}_{A}}{c^{2}}\right)
$$

so that in the slow velocity (and slow variation) limit it reduces to

$$
\delta \mathbf{a}_{A}=-c^{2} \sum_{i} f_{A, i} \nabla \alpha_{i} .
$$

where we have introduce the sensitivity of the mass $A$ with respect to the variation of the constant $\alpha_{i}$ by

$$
f_{A, i} \equiv \frac{\partial \ln m_{A}}{\partial \alpha_{i}} .
$$

This simple argument shows that if the constants depend on time then there must exist an anomalous acceleration that will depend on the chemical composition of the body $A$.

This anomalous acceleration is generated by the change in the (electromagnetic, gravitational, ...) binding energies $[152,246,386]$ but also in the Yukawa couplings and in the Higgs sector parameters so that the $\alpha_{i}$-dependencies are a priori composition-dependent. As a consequence, any variation of the fundamental constants will entail a violation of the universality of free fall: the total mass of the body being space dependent, an anomalous force appears if energy is to be conserved. The variation of the constants, deviation from general relativity and violation of the weak equivalence principle are in general expected together.

On the other hand, the composition dependence of $\delta \mathbf{a}_{A}$ and thus of $\eta_{A B}$ can be used to optimize the choice of materials for the experiments testing the equivalence principle $[118,120,122]$ but also to distinguish between several models if data from the universality of free fall and atomic clocks are combined [143]. 
From a theoretical point of view, the computation of $\eta_{A B}$ will requires the determination of the coefficients $f_{A i}$. This can be achieved in two steps by first relating the new degrees of freedom of the theory to the variation of the fundamental constants and then relating them to the variation of the masses. As we shall see in Section 5, the first issue is very model dependent while the second is especially difficult, particularly when one wants to understand the effect of the quark mass, since it is related to the intricate structure of QCD and its role in low energy nuclear reactions.

As an example, the mass of a nuclei of charge $Z$ and atomic number $A$ can be expressed as

$$
m(A, Z)=Z m_{\mathrm{p}}+(A-Z) m_{\mathrm{n}}+Z m_{\mathrm{e}}+E_{\mathrm{S}}+E_{\mathrm{EM}},
$$

where $E_{\mathrm{S}}$ and $E_{\mathrm{EM}}$ are respectively the strong and electromagnetic contributions to the binding energy. The Bethe-Weizäcker formula allows to estimate the latter as

$$
E_{\mathrm{EM}}=98.25 \frac{Z(Z-1)}{A^{1 / 3}} \alpha_{\mathrm{EM}} \mathrm{MeV} .
$$

If we decompose the proton and neutron masses as [230] $m_{(\mathrm{p}, \mathrm{n})}=u_{3}+b_{(\mathrm{u}, \mathrm{d})} m_{\mathrm{u}}+b_{(\mathrm{d}, \mathrm{u})} m_{\mathrm{d}}+$ $B_{(\mathrm{p}, \mathrm{n})} \alpha_{\mathrm{EM}}$ where $u_{3}$ is the pure QCD approximation of the nucleon mass $\left(b_{\mathrm{u}}, b_{\mathrm{d}}\right.$ and $B_{(\mathrm{n}, \mathrm{p})} / u_{3}$ being pure numbers), it reduces to

$$
\begin{aligned}
m(A, Z) & =\left(A u_{3}+E_{\mathrm{S}}\right)+\left(Z b_{\mathrm{u}}+N b_{\mathrm{d}}\right) m_{\mathrm{u}}+\left(Z b_{\mathrm{d}}+N b_{\mathrm{u}}\right) m_{\mathrm{d}} \\
& +\left(Z B_{\mathrm{p}}+N B_{\mathrm{n}}+98.25 \frac{Z(Z-1)}{A^{1 / 3}} \mathrm{MeV}\right) \alpha_{\mathrm{EM}},
\end{aligned}
$$

with $N=A-Z$, the neutron number. For an atom, one would have to add the contribution of the electrons, $Z m_{\mathrm{e}}$. This form depends on strong, weak and electromagnetic quantities. The numerical coefficients $B_{(\mathrm{n}, \mathrm{p})}$ are given explicitly by [230]

$$
B_{\mathrm{p}} \alpha_{\mathrm{EM}}=0.63 \mathrm{MeV} \quad B_{\mathrm{n}} \alpha_{\mathrm{EM}}=-0.13 \mathrm{MeV} .
$$

Such estimations were used in the first analysis of the relation between variation of the constant and the universality of free fall $[135,166]$ but the dependency on the quark mass is still not well understood and we refer to [120, 122, 157, 159, 208] for some attempts to refine this description.

For macroscopic bodies, the mass has also a negative contribution

$$
\Delta m(G)=-\frac{G}{2 c^{2}} \int \frac{\rho(\vec{r}) \rho\left(\vec{r}^{\prime}\right)}{\left|\vec{r}-\vec{r}^{\prime}\right|} \mathrm{d}^{3} \vec{r} \mathrm{~d}^{3} \vec{r}^{\prime}
$$

from the gravitational binding energy. As a conclusion, from (17) and (19), we expect the mass to depend on all the coupling constant, $m\left(\alpha_{\mathrm{EM}}, \alpha_{\mathrm{W}}, \alpha_{\mathrm{S}}, \alpha_{\mathrm{G}}, \ldots\right)$. We shall discuss this issue in more detail in Section 5.3.2.

Note that varying coupling constants can also be associated with violations of local Lorentz invariance and CPT symmetry [298, 52, 242].

\subsubsection{Relations with cosmology}

Most constraints on the time variation of the fundamental constants will not be local and related to physical systems at various epochs of the evolution of the universe. It follows that the comparison of different constraints requires a full cosmological model.

Our current cosmological model is known as the $\Lambda$ CDM (see [409] for a detailed description, and Table 4 for the typical value of the cosmological parameters). It is important to recall that its construction relies on 4 main hypotheses: (H1) a theory of gravity; (H2) a description of the matter components contained in the Universe and their non-gravitational interactions; (H3) 
symmetry hypothesis; and (H4) a hypothesis on the global structure, i.e., the topology, of the Universe. These hypotheses are not on the same footing since $\mathrm{H} 1$ and $\mathrm{H} 2$ refer to the physical theories. However, these hypotheses are not sufficient to solve the field equations and we must make an assumption on the symmetries (H3) of the solutions describing our Universe on large scales while $\mathrm{H} 4$ is an assumption on some global properties of these cosmological solutions, with same local geometry. But the last two hypothesis are unavoidable because the knowledge of the fundamental theories is not sufficient to construct a cosmological model [504].

The $\Lambda$ CDM model assumes that gravity is described by general relativity (H1), that the Universe contains the fields of the standard model of particle physics plus some dark matter and a cosmological constant, the latter two having no physical explanation at the moment. It also deeply involves the Copernican principle as a symmetry hypothesis (H3), without which the Einstein equations usually cannot been solved, and assumes most often that the spatial sections are simply connected (H4). H2 and $\mathrm{H} 3$ imply that the description of the standard matter reduces to a mixture of a pressureless and a radiation perfect fluids. This model is compatible with all astronomical data, which roughly indicates that $\Omega_{\Lambda 0} \simeq 0.73, \Omega_{\text {mat } 0} \simeq 0.27$, and $\Omega_{K 0} \simeq 0$. Thus, cosmology roughly imposes that $\left|\Lambda_{0}\right| \leq H_{0}^{2}$, that is $\ell_{\Lambda} \leq H_{0}^{-1} \sim 10^{26} \mathrm{~m} \sim 10^{41} \mathrm{GeV}^{-1}$.

Hence, the analysis of the cosmological dynamics of the universe and of its large scale structures requires the introduction of a new constant, the cosmological constant, associated with a recent acceleration of the cosmic expansion, that can be introduced by modifying the Einstein-Hilbert action to

$$
S_{\text {grav }}=\frac{c^{3}}{16 \pi G} \int \sqrt{-g}(R-2 \Lambda) \mathrm{d}^{4} x .
$$

This constant can equivalently be introduced in the matter action. Note, however, that it is disproportionately small compared to the natural scale fixed by the Planck length

$$
\rho_{\Lambda_{0}} \sim 10^{-120} M_{\mathrm{Pl}}^{4} \sim 10^{-47} \mathrm{GeV}^{4} .
$$

Classically, this value is no problem but it was pointed out that at the quantum level, the vacuum energy should scale as $M^{4}$, where $M$ is some energy scale of high-energy physics. In such a case, there is a discrepancy of $60-120$ order of magnitude between the cosmological conclusions and the theoretical expectation. This is the cosmological constant problem [528].

Two approaches to solve this problem have been considered. Either one accepts such a constant and such a fine-tuning and tries to explain it on anthropic ground. Or, in the same spirit as Dirac, one interprets it as an indication that our set of cosmological hypotheses have to be extended, by either abandoning the Copernican principle [508] or by modifying the local physical laws (either gravity or the matter sector). The way to introduce such new physical degrees of freedom were classified in [502]. In that latter approach, the tests of the constancy of the fundamental constants are central, since they can reveal the coupling of this new degree of freedom to the standard matter fields. Note, however, that the cosmological data still favor a pure cosmological constant.

Among all the proposals quintessence involves a scalar field rolling down a runaway potential hence acting as a fluid with an effective equation of state in the range $-1 \leq w \leq 1$ if the field is minimally coupled. It was proposed that the quintessence field is also the dilaton [229, 434, 499]. The same scalar field then drives the time variation of the cosmological constant and of the gravitational constant and it has the property to also have tracking solutions [499]. Such models do not solve the cosmological constant problem but only relieve the coincidence problem. One of the underlying motivation to replace the cosmological constant by a scalar field comes from superstring models in which any dimensionful parameter is expressed in terms of the string mass scale and the vacuum expectation value of a scalar field. However, the requirement of slow roll (mandatory to have a negative pressure) and the fact that the quintessence field dominates today imply, if the minimum of the potential is zero, that it is very light, roughly of order $m \sim 10^{-33} \mathrm{eV}$ [81]. 
Table 4: Main cosmological parameters in the standard $\Lambda$-CDM model. There are 7 main parameters (because $\sum \Omega_{i}=0$ ) to which one can add 6 more to include dark energy, neutrinos and gravity waves. Note that often the spatial curvature is set to $\Omega_{K}=0$. (See, e.g. Refs. [296, 409]).

\begin{tabular}{lll}
\hline Parameter & Symbol & Value \\
\hline Reduced Hubble constant & $h$ & $0.73(3)$ \\
Baryon-to-photon ratio & $\eta=n_{\mathrm{b}} / n_{\gamma}$ & $6.12(19) \times 10^{-10}$ \\
Photon density & $\Omega_{\gamma} h^{2}$ & $2.471 \times 10^{-5}$ \\
Dark matter density & $\Omega_{\mathrm{CDM}} h^{2}$ & $0.105(8)$ \\
Cosmological constant & $\Omega_{\Lambda}$ & $0.73(3)$ \\
Spatial curvature & $\Omega_{K}$ & $0.011(12)$ \\
Scalar modes amplitude & $Q$ & $(2.0 \pm 0.2) \times 10^{-5}$ \\
Scalar spectral index & $n_{S}$ & $0.958(16)$ \\
\hline Neutrino density & $\Omega_{\nu} h^{2}$ & $(0.0005-0.023)$ \\
Dark energy equation of state & $w$ & $-0.97(7)$ \\
Scalar running spectral index & $\alpha_{S}$ & $-0.05 \pm 0.03$ \\
Tensor-to-scalar ratio & $\mathrm{T} / \mathrm{S}$ & $<0.36$ \\
Tensor spectral index & $n_{T}$ & $<0.001$ \\
Tensor running spectral index & $\alpha_{T}$ & $?$ \\
\hline Baryon density & $\Omega_{\mathrm{b}} h^{2}$ & $0.0223(7)$ \\
\hline
\end{tabular}

Such a light field can lead to observable violations of the universality of free fall if it is nonuniversally coupled to the matter fields. Carroll [81] considered the effect of the coupling of this very light quintessence field to ordinary matter via a coupling to the electromagnetic field as $\phi F^{\mu \nu} \widetilde{F}_{\mu \nu}$. Chiba and Kohri [96] also argued that an ultra-light quintessence field induces a time variation of the coupling constant if it is coupled to ordinary matter and studied a coupling of the form $\phi F^{\mu \nu} F_{\mu \nu}$, as, e.g., expected from Kaluza-Klein theories (see below). This was generalized to quintessence models with a couplings of the form $Z(\phi) F^{\mu \nu} F_{\mu \nu}[11,112,162,315,314,347,404,531]$ and then to models of runaway dilaton $[133,132]$ inspired by string theory (see Section 5.4.1). The evolution of the scalar field drives both the acceleration of the universe at late time and the variation of the constants. As pointed in [96, 166, 532] such models are extremely constrained from the bound on the universality of free-fall (see Section 6.3).

We have two means of investigation:

- The field driving the time variation of the fundamental constants does not explain the acceleration of the universe (either it does not dominate the matter content today or its equation of state is not negative enough). In such a case, the variation of the constants is disconnected from the dark energy problem. Cosmology allows to determine the dynamics of this field during the whole history of the universe and thus to compare local constraints and cosmological constraints. An example is given by scalar-tensor theories (see Section 5.1.1) for which one can compare, e.g., primordial nucleosynthesis to local constraints [134]. However, in such a situation one should take into account the effect of the variation of the constants on the astrophysical observations since it can affect local physical processes and bias, e.g., the luminosity of supernovae and indirectly modify the distance luminosity-redshift relation derived from these observations [33, 435].

- The field driving the time variation of the fundamental constants is also responsible for the acceleration of the universe. It follows that the dynamics of the universe, the level of variation of the constants and the other deviations from general relativity are connected [348] so that the study of the variation of the constants can improve the reconstruction of the equation 
state of the dark energy [20, 162, 389, 404].

In conclusion, cosmology seems to require a new constant. It also provides a link between the microphysics and cosmology, as foreseen by Dirac. The tests of fundamental constants can discriminate between various explanations of the acceleration of the universe. When a model is specified, cosmology also allows to set stringer constraints since it relates observables that cannot be compared otherwise. 


\section{Experimental and Observational Constraints}

This section focuses on the experimental and observational constraints on the non-gravitational constants, that is assuming $\alpha_{\mathrm{G}}$ remains constant. We use the convention that $\Delta \alpha=\alpha-\alpha_{0}$ for any constant $\alpha$, so that $\Delta \alpha<0$ refers to a value smaller than today.

The various physical systems that have been considered can be classified in many ways. We can classify them according to their look-back time and more precisely their space-time position relative to our actual position. This is summarized in Figure 1. Indeed higher redshift systems offer the possibility to set constraints on a larger time scale, but this is at the expense of usually involving other parameters such as the cosmological parameters. This is, in particular, the case of the cosmic microwave background or of primordial nucleosynthesis. The systems can also be classified in terms of the physics they involve. For instance, atomics clocks, quasar absorption spectra and the cosmic microwave background require only to use quantum electrodynamics to draw the primary constraints while the Oklo phenomenon, meteorites dating and nucleosynthesis require nuclear physics.

For any system, setting constraints goes through several steps. First we have some observable quantities from which we can draw constraints on primary constants, which may not be fundamental constants (e.g., the BBN parameters, the lifetime of $\beta$-decayers, ... ). These primary parameters must then be related to some fundamental constants such as masses and couplings. In a last step, the number of constants can be reduced by relating them in some unification schemes. Indeed each step requires a specific modelization and hypothesis and has its own limitations. This is summarized on Table 5 .

Table 5: Summary of the systems considered to set constraints on the variation of the fundamental constants. We summarize the observable quantities, the primary constants used to interpret the data and the other hypothesis required for this interpretation. All the quantities appearing in this table are defined in the text.

\begin{tabular}{llll}
\hline System & Observable & Primary constraints & Other hypothesis \\
\hline Atomic clock & $\delta \ln \nu$ & $g_{i}, \alpha_{\mathrm{EM}}, \mu$ & - \\
Oklo phenomenon & isotopic ratio & $E_{r}$ & geophysical model \\
Meteorite dating & isotopic ratio & $\lambda$ & - \\
Quasar spectra & atomic spectra & $g_{\mathrm{p}}, \mu, \alpha_{\mathrm{EM}}$ & cloud physical properties \\
Stellar physics & element abundances & $B_{D}$ & stellar model \\
$21 \mathrm{~cm}$ & $T_{b} / T_{\mathrm{CMB}}$ & $g_{\mathrm{p}}, \mu, \alpha_{\mathrm{EM}}$ & cosmological model \\
CMB & $\Delta T / T$ & $\mu, \alpha_{\mathrm{EM}}$ & cosmological model \\
BBN & light element abundances & $Q_{\mathrm{np}}, \tau_{\mathrm{n}}, m_{\mathrm{e}}, m_{\mathrm{N}}, \alpha_{\mathrm{EM}}, B_{D}$ & cosmological model \\
\hline
\end{tabular}

\subsection{Atomic clocks}

\subsubsection{Atomic spectra and constants}

The laboratory constraints on the time variation of fundamental constants are obtained by comparing the long-term behavior of several oscillators and rely on frequency measurements. The atomic transitions have various dependencies in the fundamental constants. For instance, for the hydrogen atom, the gross, fine and hyperfine-structures are roughly given by

$$
2 p-1 s: \nu \propto c R_{\infty}, \quad 2 p_{3 / 2}-2 p_{1 / 2}: \nu \propto c R_{\infty} \alpha_{\mathrm{EM}}^{2}, \quad 1 s: \propto c R_{\infty} \alpha_{\mathrm{EM}}^{2} g_{\mathrm{p}} \bar{\mu}
$$

respectively, where the Rydberg constant set the dimension. $g_{\mathrm{p}}$ is the proton gyromagnetic factor and $\bar{\mu}=m_{\mathrm{e}} / m_{\mathrm{p}}$. In the non-relativistic approximation, the transitions of all atoms have similar 

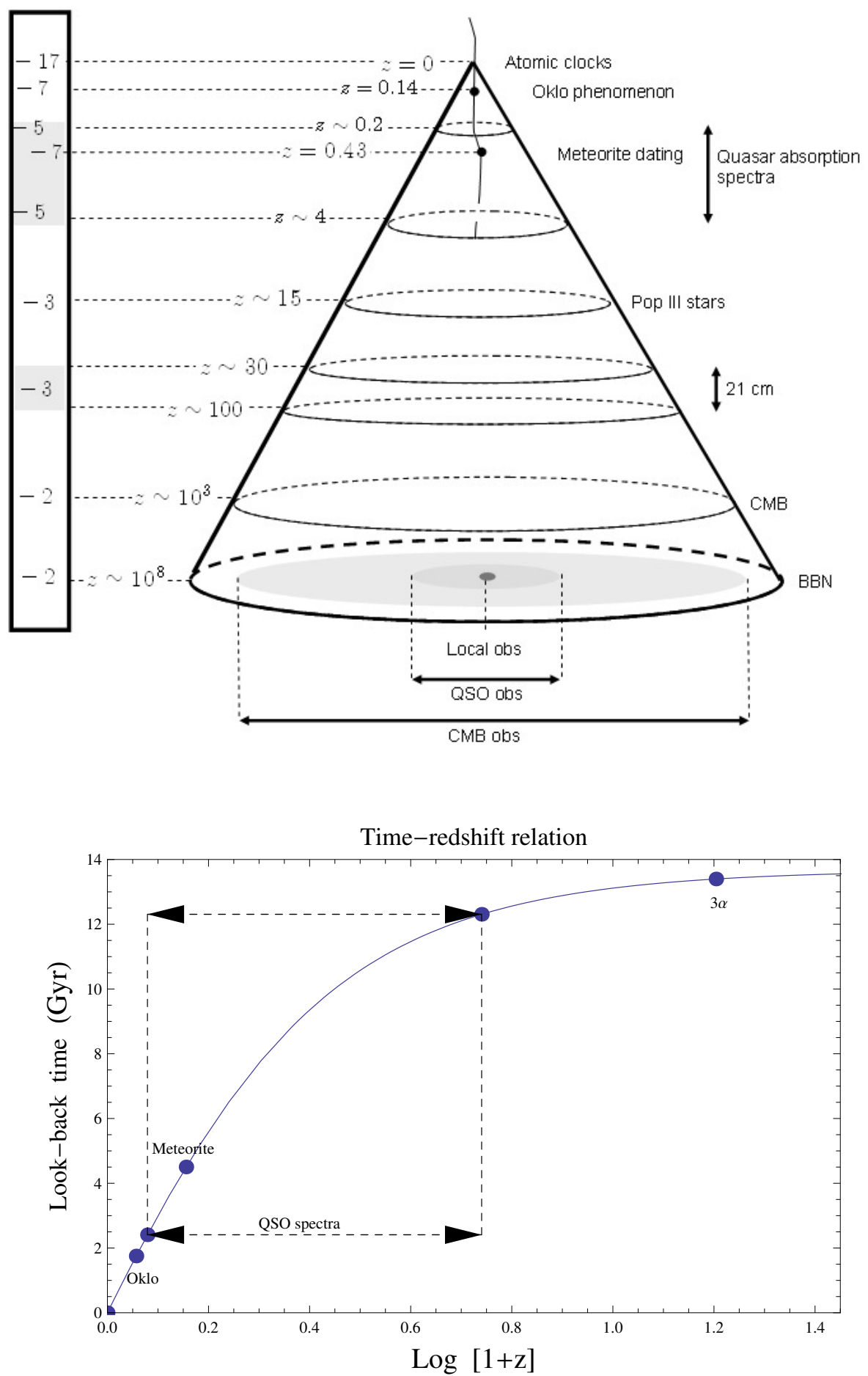

Figure 1: Top: Summary of the systems that have been used to probe the constancy of the fundamental constants and their position in a space-time diagram in which the cone represents our past light cone. The shaded areas represents the comoving space probed by different tests. Bottom: The look-back time-redshift relation for the standard $\Lambda \mathrm{CDM}$ model. 
dependencies but two effects have to be taken into account. First, the hyperfine-structures involve a gyromagnetic factor $g_{i}$ (related to the nuclear magnetic moment by $\mu_{i}=g_{i} \mu_{\mathrm{N}}$, with $\mu_{\mathrm{N}}=$ $\left.e \hbar / 2 m_{\mathrm{p}} c\right)$, which are different for each nuclei. Second, relativistic corrections (including the Casimir contribution), which also depend on each atom (but also on the type of the transition) can be included through a multiplicative function $F_{\text {rel }}\left(\alpha_{\mathrm{EM}}\right)$. It has a strong dependence on the atomic number $Z$, which can be illustrated on the case of alkali atoms, for which

$$
F_{\text {rel }}\left(\alpha_{\mathrm{EM}}\right)=\left[1-\left(Z \alpha_{\mathrm{EM}}\right)^{2}\right]^{-1 / 2}\left[1-\frac{4}{3}\left(Z \alpha_{\mathrm{EM}}\right)^{2}\right]^{-1} \simeq 1+\frac{11}{6}\left(Z \alpha_{\mathrm{EM}}\right)^{2}
$$

The developments of highly accurate atomic clocks using different transitions in different atoms offer the possibility to test a variation of various combinations of the fundamental constants.

It follows that at the lowest level of description, we can interpret all atomic clocks results in terms of the g-factors of each atoms, $g_{i}$, the electron to proton mass ration $\mu$ and the fine-structure constant $\alpha_{\mathrm{EM}}$. We shall parameterize the hyperfine and fine-structures frequencies as follows.

The hyperfine frequency in a given electronic state of an alkali-like atom, such as ${ }^{133} \mathrm{Cs},{ }^{87} \mathrm{Rb}$, ${ }^{199} \mathrm{Hg}^{+}$, is

$$
\nu_{\mathrm{hfs}} \simeq R_{\infty} c \times A_{\mathrm{hfs}} \times g_{i} \times \alpha_{\mathrm{EM}}^{2} \times \bar{\mu} \times F_{\mathrm{hfs}}(\alpha)
$$

where $g_{i}=\mu_{i} / \mu_{\mathrm{N}}$ is the nuclear $g$ factor. $A_{\mathrm{hfs}}$ is a numerical factor depending on each particular atom and we have set $F_{\text {rel }}=F_{\mathrm{hfs}}(\alpha)$. Similarly, the frequency of an electronic transition is wellapproximated by

$$
\nu_{\mathrm{elec}} \simeq R_{\infty} c \times A_{\mathrm{elec}} \times F_{\mathrm{elec}}(Z, \alpha),
$$

where, as above, $A_{\text {elec }}$ is a numerical factor depending on each particular atom and $F_{\text {elec }}$ is the function accounting for relativistic effects, spin-orbit couplings and many-body effects. Even though an electronic transition should also include a contribution from the hyperfine interaction, it is generally only a small fraction of the transition energy and thus should not carry any significant sensitivity to a variation of the fundamental constants.

The importance of the relativistic corrections was probably first emphasized in [423] and their computation through relativistic $N$-body calculations was carried out for many transitions in [170, 174, 175, 198]. They can be characterized by introducing the sensitivity of the relativistic factors to a variation of $\alpha_{\mathrm{EM}}$,

$$
\kappa_{\alpha} \equiv \frac{\partial \ln F}{\partial \ln \alpha_{\mathrm{EM}}} .
$$

Table 6 summarizes the values of some of them, as computed in [175, 210]. Indeed a reliable knowledge of these coefficients at the $1 \%$ to $10 \%$ level is required to deduce limits to a possible variation of the constants. The interpretation of the spectra in this context relies, from a theoretical point of view, only on quantum electrodynamics (QED), a theory, which is well tested experimentally [280] so that we can safely obtain constraints on $\left(\alpha_{\mathrm{EM}}, \mu, g_{i}\right)$, still keeping in mind that the computation of the sensitivity factors required numerical $N$-body simulations.

From an experimental point of view, various combinations of clocks have been performed. It is important to analyze as many species as possible in order to rule-out species-dependent systematic effects. Most experiments are based on a frequency comparison to caesium clocks. The hyperfine splitting frequency between the $F=3$ and $F=4$ levels of its ${ }^{2} S_{1 / 2}$ ground state at $9.192 \mathrm{GHz}$ has been used for the definition of the second since 1967. One limiting effect, that contributes mostly to the systematic uncertainty, is the frequency shift due to cold collisions between the atoms. On this particular point, clocks based on the hyperfine frequency of the ground state of the rubidium at $6.835 \mathrm{GHz}$, are more favorable.

Living Reviews in Relativity

http://www. livingreviews.org/lrr-2011-2 
Table 6: Sensitivity of various transitions on a variation of the fine-structure constant.

\begin{tabular}{lcc}
\hline Atom & Transition & sensitivity $\kappa_{\alpha}$ \\
\hline${ }^{1} \mathrm{H}$ & $1 s-2 s$ & 0.00 \\
${ }^{87} \mathrm{Rb}$ & $\mathrm{hf}$ & 0.34 \\
${ }^{133} \mathrm{Cs}$ & ${ }^{2} S_{1 / 2}(F=2)-(F=3)$ & 0.83 \\
${ }^{171} \mathrm{Yb}^{+}$ & $2 S_{1 / 2}-{ }^{2} D_{3 / 2}$ & 0.9 \\
${ }^{199} \mathrm{Hg}^{+}$ & ${ }^{2} S_{1 / 2}-{ }^{2} D_{5 / 2}$ & -3.2 \\
${ }^{87} \mathrm{Sr}^{1} S_{0}-{ }^{3} P_{0}$ & 0.06 \\
${ }^{27} \mathrm{Al}^{+}$ & ${ }^{1} S_{0}-{ }^{3} P_{0}$ & 0.008 \\
\hline
\end{tabular}

\subsubsection{Experimental constraints}

We present the latest results that have been obtained and refer to Section III.B.2 of FCV [500] for earlier studies. They all rely on the developments of new atomic clocks, with the primarily goal to define better frequency standards.

Table 7: Summary of the constraints obtained from the comparisons of atomic clocks. For each constraint on the relative drift of the frequency of the two clocks, we provide the dependence in the various constants, using the numbers of Table 6. From Ref. [379], which can be consulted for other constants.

\begin{tabular}{|c|c|c|c|c|}
\hline Clock 1 & $\begin{array}{c}\text { Clock } 2 \\
\frac{\mathrm{d}}{\mathrm{d} t} \ln \left(\frac{\nu_{\text {clock } 1}}{\nu_{\text {clock } 2}}\right)\end{array}$ & Constraint $\left(\mathrm{yr}^{-1}\right)$ & Constants dependence & Reference \\
\hline${ }^{87} \mathrm{Rb}$ & ${ }^{133} \mathrm{Cs}$ & $(0.2 \pm 7.0) \times 10^{-16}$ & $\frac{g_{\mathrm{Cs}}}{g_{\mathrm{D}}} \alpha_{\mathrm{EM}}^{0.49}$ & [346] \\
\hline${ }^{87} \mathrm{Rb}$ & ${ }^{133} \mathrm{Cs}$ & $(-0.5 \pm 5.3) \times 10^{-16}$ & & {$[58]$} \\
\hline${ }^{1} \mathrm{H}$ & ${ }^{133} \mathrm{Cs}$ & $(-32 \pm 63) \times 10^{-16}$ & $g_{\mathrm{Cs}} \bar{\mu} \alpha_{\mathrm{EM}}^{2.83}$ & [196] \\
\hline${ }^{199} \mathrm{Hg}^{+}$ & ${ }^{133} \mathrm{Cs}$ & $(0.2 \pm 7) \times 10^{-15}$ & $g_{\mathrm{Cs}} \bar{\mu} \alpha_{\mathrm{EM}}^{6.05}$ & {$[57]$} \\
\hline${ }^{199} \mathrm{Hg}^{+}$ & ${ }^{133} \mathrm{Cs}$ & $(3.7 \pm 3.9) \times 10^{-16}$ & & [214] \\
\hline${ }^{171} \mathrm{Yb}^{+}$ & ${ }^{133} \mathrm{Cs}$ & $(-1.2 \pm 4.4) \times 10^{-15}$ & $g_{\mathrm{Cs}} \bar{\mu} \alpha_{\mathrm{EM}}^{1.93}$ & [408] \\
\hline${ }^{171} \mathrm{Yb}^{+}$ & ${ }^{133} \mathrm{Cs}$ & $(-0.78 \pm 1.40) \times 10^{-15}$ & & {$[407]$} \\
\hline${ }^{87} \mathrm{Sr}$ & ${ }^{133} \mathrm{Cs}$ & $(-1.0 \pm 1.8) \times 10^{-15}$ & $g_{\mathrm{Cs}} \bar{\mu} \alpha_{\mathrm{EM}}^{2.77}$ & {$[61]$} \\
\hline${ }^{87} \mathrm{Dy}$ & ${ }^{87} \mathrm{Dy}$ & $(-2.7 \pm 2.6) \times 10^{-15}$ & $\alpha_{\mathrm{EM}}$ & {$[100]$} \\
\hline${ }^{27} \mathrm{Al}^{+}$ & ${ }^{199} \mathrm{Hg}^{+}$ & $(-5.3 \pm 7.9) \times 10^{-17}$ & $\alpha_{\mathrm{EM}}^{-3.208}$ & {$[440]$} \\
\hline
\end{tabular}

- Rubidium: The comparison of the hyperfine frequencies of the rubidium and caesium in their electronic ground state between 1998 and 2003, with an accuracy of order $10^{-15}$, leads to the constraint [346]

$$
\frac{\mathrm{d}}{\mathrm{d} t} \ln \left(\frac{\nu_{\mathrm{Rb}}}{\nu_{\mathrm{Cs}}}\right)=(0.2 \pm 7.0) \times 10^{-16} \mathrm{yr}^{-1}
$$

With one more year of experiment, the constraint dropped to [58]

$$
\frac{\mathrm{d}}{\mathrm{d} t} \ln \left(\frac{\nu_{\mathrm{Rb}}}{\nu_{\mathrm{Cs}}}\right)=(-0.5 \pm 5.3) \times 10^{-16} \mathrm{yr}^{-1} \text {. }
$$

From Equation (21), and using the values of the sensitivities $\kappa_{\alpha}$, we deduce that comparison constrains

$$
\frac{\nu_{\mathrm{Cs}}}{\nu_{\mathrm{Rb}}} \propto \frac{g_{\mathrm{Cs}}}{g_{\mathrm{Rb}}} \alpha_{\mathrm{EM}}^{0.49}
$$




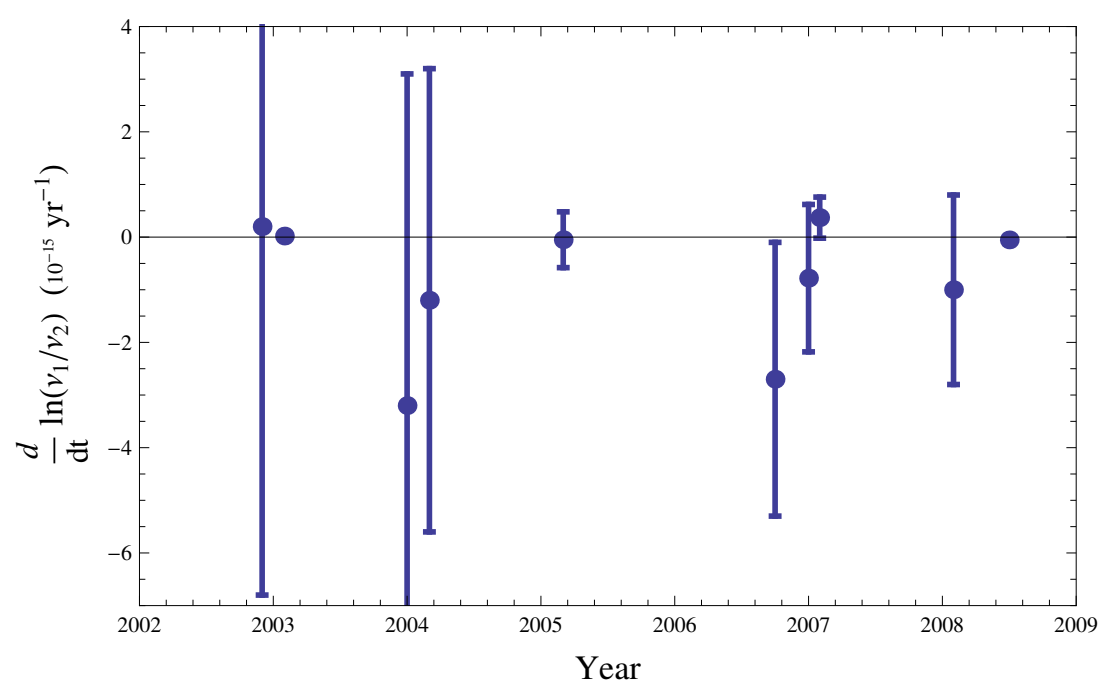

Figure 2: Evolution of the comparison of different atomic clocks summarized in Table 7.

- Atomic hydrogen: The $1 s-2 s$ transition in atomic hydrogen was compared tp the ground state hyperfine splitting of caesium [196] in 1999 and 2003, setting an upper limit on the variation of $\nu_{\mathrm{H}}$ of $(-29 \pm 57) \mathrm{Hz}$ within 44 months. This can be translated in a relative drift

$$
\frac{\mathrm{d}}{\mathrm{d} t} \ln \left(\frac{\nu_{\mathrm{H}}}{\nu_{\mathrm{Cs}}}\right)=(-32 \pm 63) \times 10^{-16} \mathrm{yr}^{-1}
$$

Since the relativistic correction for the atomic hydrogen transition nearly vanishes, we have $\nu_{\mathrm{H}} \sim R_{\infty}$ so that

$$
\frac{\nu_{\mathrm{Cs}}}{\nu_{\mathrm{H}}} \propto g_{\mathrm{Cs}} \bar{\mu} \alpha_{\mathrm{EM}}^{2.83}
$$

- Mercury: The ${ }^{199} \mathrm{Hg}^{+}{ }^{2} S_{1 / 2}-{ }^{2} D_{5 / 2}$ optical transition has a high sensitivity to $\alpha_{\mathrm{EM}}$ (see Table 6) so that it is well suited to test its variation. The frequency of the ${ }^{199} \mathrm{Hg}^{+}$electric quadrupole transition at $282 \mathrm{~nm}$ was compared to the ground state hyperfine transition of caesium during a two year period, which lead to [57]

$$
\frac{\mathrm{d}}{\mathrm{d} t} \ln \left(\frac{\nu_{\mathrm{Hg}}}{\nu_{\mathrm{Cs}}}\right)=(0.2 \pm 7) \times 10^{-15} \mathrm{yr}^{-1} .
$$

This was improved by a comparison over a 6 year period [214] to get

$$
\frac{\mathrm{d}}{\mathrm{d} t} \ln \left(\frac{\nu_{\mathrm{Hg}}}{\nu_{\mathrm{Cs}}}\right)=(3.7 \pm 3.9) \times 10^{-16} \mathrm{yr}^{-1}
$$

While $\nu_{\mathrm{Cs}}$ is still given by Equation (21), $\nu_{\mathrm{Hg}}$ is given by Equation (22). Using the sensitivities of Table 6 , we conclude that this comparison test the stability of

$$
\frac{\nu_{\mathrm{Cs}}}{\nu_{\mathrm{Hg}}} \propto g_{\mathrm{Cs}} \bar{\mu} \alpha_{\mathrm{EM}}^{6.05}
$$

- Ytterbium: The ${ }^{2} S_{1 / 2}-{ }^{2} D_{3 / 2}$ electric quadrupole transition at $688 \mathrm{THz}$ of ${ }^{171} \mathrm{Yb}^{+}$was compared to the ground state hyperfine transition of cesium. The constraint of [408] was 
updated, after comparison over a six year period, which lead to [407]

$$
\frac{\mathrm{d}}{\mathrm{d} t} \ln \left(\frac{\nu_{\mathrm{Yb}}}{\nu_{\mathrm{Cs}}}\right)=(-0.78 \pm 1.40) \times 10^{-15} \mathrm{yr}^{-1} .
$$

Proceeding as previously, this tests the stability of

$$
\frac{\nu_{\mathrm{Cs}}}{\nu_{\mathrm{Yb}}} \propto g_{\mathrm{Cs}} \bar{\mu} \alpha_{\mathrm{EM}}^{1.93} \text {. }
$$

- Strontium: The comparison of the ${ }^{1} S_{0}-{ }^{3} P_{0}$ transition in neutral ${ }^{87} \mathrm{Sr}$ with a cesium clock was performed in three independent laboratories. The combination of these three experiments [61] leads to the constraint

$$
\frac{\mathrm{d}}{\mathrm{d} t} \ln \left(\frac{\nu_{\mathrm{Sr}}}{\nu_{\mathrm{Cs}}}\right)=(-1.0 \pm 1.8) \times 10^{-15} \mathrm{yr}^{-1} .
$$

Proceeding as previously, this tests the stability of

$$
\frac{\nu_{\mathrm{Cs}}}{\nu_{\mathrm{Sr}}} \propto g_{\mathrm{Cs}} \bar{\mu} \alpha_{\mathrm{EM}}^{2.77} .
$$

- Atomic dyprosium: It was suggested in $[175,174]$ (see also [173] for a computation of the transition amplitudes of the low states of dyprosium) that the electric dipole (E1) transition between two nearly degenerate opposite-parity states in atomic dyprosium should be highly sensitive to the variation of $\alpha_{\mathrm{EM}}$. It was then demonstrated [384] that a constraint of the order of $10^{-18} / \mathrm{yr}$ can be reached. The frequencies of nearly of two isotopes of dyprosium were monitored over a 8 months period [100] showing that the frequency variation of the $3.1-\mathrm{MHz}$ transition in ${ }^{163} \mathrm{Dy}$ and the $235-\mathrm{MHz}$ transition in ${ }^{162} \mathrm{Dy}$ are $9.0 \pm 6.7 \mathrm{~Hz} / \mathrm{yr}$ and $-0.6 \pm 6.5 \mathrm{~Hz} / \mathrm{yr}$, respectively. These provide the constraint

$$
\frac{\alpha_{\mathrm{EM}}}{\alpha_{\mathrm{EM}}}=(-2.7 \pm 2.6) \times 10^{-15} \mathrm{yr}^{-1},
$$

at $1 \sigma$ level, without any assumptions on the constancy of other fundamental constants.

- Aluminium and mercury single-ion optical clocks: The comparison of the ${ }^{1} S_{0}-{ }^{3} P_{0}$ transition in ${ }^{27} \mathrm{Al}^{+}$and ${ }^{2} S_{1 / 2}-{ }^{2} D_{5 / 2}$ in ${ }^{199} \mathrm{Hg}^{+}$over a year allowed to set the constraint [440]

$$
\frac{\mathrm{d}}{\mathrm{d} t} \ln \left(\frac{\nu_{\mathrm{Al}}}{\nu_{\mathrm{Hg}}}\right)=(-5.3 \pm 7.9) \times 10^{-17} \mathrm{yr}^{-1} .
$$

Proceeding as previously, this tests the stability of

$$
\frac{\nu_{\mathrm{Hg}}}{\nu_{\mathrm{Al}}} \propto \alpha_{\mathrm{EM}}^{-3.208},
$$

which directly set the constraint

$$
\frac{\alpha_{\mathrm{EM}}}{\alpha_{\mathrm{EM}}}=(-1.6 \pm 2.3) \times 10^{-17} \mathrm{yr}^{-1},
$$

since it depends only on $\alpha_{\mathrm{EM}}$. 
While the constraint (33) was obtained directly from the clock comparison, the other studies need to be combined to disentangle the contributions of the various constants. As an example, we first use the bound (33) on $\alpha_{\mathrm{EM}}$, we can then extract the two following bounds

$$
\frac{\mathrm{d}}{\mathrm{d} t} \ln \left(\frac{g_{\mathrm{Cs}}}{g_{\mathrm{Rb}}}\right)=(0.48 \pm 6.68) \times 10^{-16} \mathrm{yr}^{-1}, \quad \frac{\mathrm{d}}{\mathrm{d} t} \ln \left(g_{\mathrm{Cs}} \bar{\mu}\right)=(4.67 \pm 5.29) \times 10^{-16} \mathrm{yr}^{-1}
$$

on a time scale of a year. We cannot lift the degeneracies further with this clock comparison, since that would require a constraint on the time variation of $\mu$. All these constraints are summarized in Table 7 and Figure 2.

A solution is to consider diatomic molecules since, as first pointed out by Thomson [488], molecular lines can provide a test of the variation of $\mu$. The energy difference between two adjacent rotational levels in a diatomic molecule is inversely proportional to $M r^{-2}, r$ being the bond length and $M$ the reduced mass, and the vibrational transition of the same molecule has, in first approximation, a $\sqrt{M}$ dependence. For molecular hydrogen $M=m_{\mathrm{p}} / 2$ so that the comparison of an observed vibro-rotational spectrum with a laboratory spectrum gives an information on the variation of $m_{\mathrm{p}}$ and $m_{\mathrm{n}}$. Comparing pure rotational transitions with electronic transitions gives a measurement of $\mu$. It follows that the frequency of vibro-rotation transitions is, in the Born-Oppenheimer approximation, of the form

$$
\nu \simeq E_{I}\left(c_{\mathrm{elec}}+c_{\mathrm{vib}} \sqrt{\bar{\mu}}+c_{\mathrm{rot}} \bar{\mu}\right)
$$

where $c_{\text {elec }}, c_{\mathrm{vib}}$ and $c_{\text {rot }}$ are some numerical coefficients.

The comparison of the vibro-rotational transition in the molecule SF6 was compared to a caesium clock over a two-year period, leading to the constraint [464]

$$
\frac{\mathrm{d}}{\mathrm{d} t} \ln \left(\frac{\nu_{\mathrm{SF} 6}}{\nu_{\mathrm{Cs}}}\right)=(1.9 \pm 0.12 \pm 2.7) \times 10^{-14} \mathrm{yr}^{-1},
$$

where the second error takes into account uncontrolled systematics. Now, using again Table 6, we deduce that

$$
\frac{\nu_{\mathrm{SF} 6}}{\nu_{\mathrm{Cs}}} \propto \bar{\mu}^{1 / 2} \alpha_{\mathrm{EM}}^{-2.83}\left(g_{\mathrm{Cs}} \bar{\mu}\right)^{-1} .
$$

It can be combined with the constraint (26), which enjoys the same dependence to cesium to infer that

$$
\frac{\dot{\mu}}{\mu}=(-3.8 \pm 5.6) \times 10^{-14} \mathrm{yr}^{-1}
$$

Combined with Equation (34), we can obtain independent constraints on the time variation of $g_{\mathrm{Cs}}$, $g_{\mathrm{Rb}}$ and $\mu$.

\subsubsection{Physical interpretation}

The theoretical description must be pushed further if ones wants to extract constraints on constant more fundamental than the nuclear magnetic moments. This requires one to use quantum chromodynamics. In particular, it was argued than within this theoretical framework, one can relate the nucleon $g$-factors in terms of the quark mass and the QCD scale [198]. Under the assumption of a unification of the three non-gravitational interaction (see Section 6.3), the dependence of the magnetic moments on the quark masses was investigated in [210]. The magnetic moments, or equivalently the $g$-factors, are first related to the ones of the proton and a neutron to derive a relation of the form

$$
g \propto g_{\mathrm{p}}^{a_{\mathrm{p}}} g_{\mathrm{n}}^{a_{\mathrm{n}}}
$$


$[198,210]$ argued that these $g$-factors mainly depend on the light quark mass $m_{\mathrm{q}}=\frac{1}{2}\left(m_{\mathrm{u}}+m_{\mathrm{d}}\right)$ and $m_{\mathrm{s}}$, respectively for the up, down and strange quarks, that is in terms of $X_{\mathrm{q}}=m_{\mathrm{q}} / \Lambda_{\mathrm{QCD}}$ and $X_{\mathrm{s}}=m_{\mathrm{s}} / \Lambda_{\mathrm{QCD}}$. Using a chiral perturbation theory, it was deduced, assuming $\Lambda_{\mathrm{QCD}}$ constant, that

$$
g_{\mathrm{p}} \propto X_{\mathrm{q}}^{-0.087} X_{\mathrm{s}}^{-0.013}, \quad g_{\mathrm{n}} \propto X_{\mathrm{q}}^{-0.118} X_{\mathrm{s}}^{0.0013},
$$

so that for a hyperfine transition

$$
\nu_{\mathrm{hfs}} \propto \alpha_{\mathrm{EM}}^{2+\kappa_{\alpha}} X_{\mathrm{q}}^{\kappa_{\mathrm{q}}} X_{\mathrm{s}}^{\kappa_{\mathrm{s}}} \bar{\mu} .
$$

Both coefficients can be computed, leading to the possibility to draw constraints on the independent time variation of $X_{\mathrm{q}}, X_{\mathrm{s}}$ and $X_{\mathrm{e}}$.

To simplify, we may assume that $X_{\mathrm{q}} \propto X_{\mathrm{s}}$, which is motivated by the Higgs mechanism of mass generation, so that the dependence in the quark masses reduces to $\kappa=\frac{1}{2}\left(\kappa_{\mathrm{q}}+\kappa_{\mathrm{s}}\right)$. For instance, we have

$$
\kappa_{\mathrm{Cs}}=0.009, \quad \kappa_{\mathrm{Rb}}=-0.016, \quad \kappa_{\mathrm{H}}=-0.10 .
$$

For hyperfine transition, one further needs to take into account the dependence in $\mu$ that can be described [204] by

$$
m_{\mathrm{p}} \sim 3 \Lambda_{\mathrm{QCD}} X_{\mathrm{q}}^{0.037} X_{\mathrm{s}}^{0.011},
$$

so that the hyperfine frequencies behaves as

$$
\nu_{\mathrm{hfs}} \propto \alpha_{\mathrm{EM}}^{2+\kappa_{\alpha}} X_{\mathrm{q}}^{\kappa-0.048} X_{\mathrm{e}},
$$

in the approximation $X_{\mathrm{q}} \propto X_{\mathrm{s}}$ and where $X_{\mathrm{e}} \equiv m_{\mathrm{e}} / \Lambda_{\mathrm{QCD}}$. This allows one to get independent constraints on the independent time variation of $X_{\mathrm{e}}, X_{\mathrm{q}}$ and $\alpha_{\mathrm{EM}}$. Indeed, these constraints are model-dependent and, as an example, Table III of [210] compares the values of the sensitivity $\kappa$ when different nuclear effects are considered. For instance, it can vary from $0.127,0.044$ to 0.009 for the cesium according to whether one includes only valence nucleon, non-valence non-nucleon or effect of the quark mass on the spin-spin interaction. Thus, it is a very promising framework, which still needs to be developed and the accuracy of which must be quantified in detail.

\subsubsection{Future evolutions}

Further progresses in a near future are expected mainly through three types of developments:

- New systems: Many new systems with enhanced sensitivity [171, 200, 202, 205, 421] to some fundamental constants have recently been proposed. Other atomic systems are considered, such as, e.g., the hyperfine transitions in the electronic ground state of cold, trapped, hydrogen-like highly charged ions [44, 199, 448], or ultra-cold atom and molecule systems near the Feshbach resonances [98], where the scattering length is extremely sensitive to $\mu$.

Concerning diatomic molecules, it was shown that this sensitivity can be enhanced in transitions between narrow close levels of different nature [13, 15]. In such transitions, the fine structure mainly depends on the fine-structure constant, $\nu_{\mathrm{fs}} \sim\left(Z \alpha_{\mathrm{EM}}\right)^{2} R_{\infty} c$, while the vibrational levels depend mainly on the electron-to-proton mass ratio and the reduced mass of the molecule, $\nu_{\mathrm{v}} \sim M_{r}^{-1 / 2} \bar{\mu}^{1 / 2} R_{\infty} c$. There could be a cancellation between the two frequencies when $\nu=\nu_{\mathrm{hf}}-n \nu_{\mathrm{v}} \sim 0$ with $n$ a positive integer. It follows that $\delta \nu / \nu$ will be proportional to $K=\nu_{\mathrm{hf}} / \nu$ so that the sensitivity to $\alpha_{\mathrm{EM}}$ and $\mu$ can be enhanced for these particular transitions. A similar effect between transistions with hyperfine-structures, for which the sensitivity to $\alpha_{\mathrm{EM}}$ can reach 600 for instance for ${ }^{139} \mathrm{La}^{32} \mathrm{~S}$ or silicon monobrid [42] that allows one to constrain $\alpha_{\mathrm{EM}} \bar{\mu}^{-1 / 4}$. 
Nuclear transitions, such as an optical clock based on a very narrow ultraviolet nuclear transition between the ground and first excited states in the ${ }^{229} \mathrm{Th}$, are also under consideration. Using a Walecka model for the nuclear potential, it was concluded [199] that the sensitivity of the transition to the fine-structure constant and quark mass was typically

$$
\frac{\delta \omega}{\omega} \sim 10^{5}\left(4 \frac{\delta \alpha_{\mathrm{EM}}}{\alpha_{\mathrm{EM}}}+\frac{\delta X_{\mathrm{q}}}{X_{\mathrm{q}}}-10 \frac{\delta X_{\mathrm{s}}}{X_{\mathrm{s}}}\right),
$$

which roughly provides a five order of magnitude amplification, which can lead to a constraint at the level of $10^{-24} / \mathrm{yr}$ on the time variation of $X_{\mathrm{q}}$. Such a method is promising and would offer different sensitivities to systematic effects compared to atomic clocks. However, this sensitivity is not clearly established since different nuclear calculations do not agree [46, 247].

- Atomic clocks in space (ACES): An improvement of at least an order of magnitude on current constraints can be achieved in space with the PHARAO/ACES project [433, 444] of the European Spatial Agency. PHARAO (Projet d'Horloge Atomique par Refroidissement d'Atomes en Orbite) combines laser cooling techniques and a microgravity environment in a satellite orbit. It aims at achieving time and frequency transfer with stability better than $10^{-16}$.

The SAGAS (Search for anomalous gravitation using atomic sensor) project aims at flying highly sensitive optical atomic clocks and cold atom accelerometers on a solar system trajectory on a time scale of 10 years. It could test the constancy of the fine-structure constant along the satellite worldline, which, in particular, can set a constraint on its spatial variation of the order of $10^{-9}[433,547]$.

- Theoretical developments: We remind one more time that the interpretation of the experiments requires a good theoretical understanding of the systems but also that the constraints we draw on the fundamental constants such as the quark masses are conditional to our theoretical modeling, hence on hypothesis on a unification scheme as well as nuclear physics. The accuracy and the robustness of these steps need to be determined, e.g., by taking the dependence in the nuclear radius [154].

\subsection{The Oklo phenomenon}

\subsubsection{A natural nuclear reactor}

Oklo is the name of a town in the Gabon republic (West Africa) where an open-pit uranium mine is situated. About $1.8 \times 10^{9} \mathrm{yr}$ ago (corresponding to a redshift of $\sim 0.14$ with the cosmological concordance model), in one of the rich vein of uranium ore, a natural nuclear reactor went critical, consumed a portion of its fuel and then shut a few million years later (see, e.g., [509] for more details). This phenomenon was discovered by the French Commissariat à l'Énergie Atomique in 1972 while monitoring for uranium ores [382]. Sixteen natural uranium reactors have been identified. Well studied reactors include the zone RZ2 (about 60 bore-holes, $1800 \mathrm{~kg}$ of ${ }^{235} \mathrm{U}$ fissioned during $8.5 \times 10^{5} \mathrm{yr}$ ) and zone RZ10 (about 13 bore-holes, $650 \mathrm{~kg}$ of ${ }^{235} \mathrm{U}$ fissioned during $\left.1.6 \times 10^{5} \mathrm{yr}\right)$.

The existence of such a natural reactor was predicted by P. Kuroda [303] who showed that under favorable conditions, a spontaneous chain reaction could take place in rich uranium deposits. Indeed, two billion years ago, uranium was naturally enriched (due to the difference of decay rate between ${ }^{235} \mathrm{U}$ and ${ }^{238} \mathrm{U}$ ) and ${ }^{235} \mathrm{U}$ represented about $3.68 \%$ of the total uranium (compared with $0.72 \%$ today and to the $3-5 \%$ enrichment used in most commercial reactors). Besides, in Oklo the conditions were favorable: (1) the concentration of neutron absorbers, which prevent the neutrons from being available for the chain fission, was low; (2) water played the role of moderator (the

Living Reviews in Relativity

http://www. livingreviews . org//rr-2011-2 
zones RZ2 and RZ10 operated at a depth of several thousand meters, so that the water pressure and temperature was close to the pressurized water reactors of $20 \mathrm{Mpa}$ and $300^{\circ} \mathrm{C}$ ) and slowed down fast neutrons so that they can interact with other ${ }^{235} \mathrm{U}$ and (3) the reactor was large enough so that the neutrons did not escape faster than they were produced. It is estimated that the Oklo reactor powered 10 to $50 \mathrm{~kW}$. This explanation is backed up by the substantial depletion of ${ }^{235} \mathrm{U}$ as well as a correlated peculiar distribution of some rare-earth isotopes. These rare-earth isotopes are abundantly produced during the fission of uranium and, in particular, the strong neutron absorbers like ${ }_{62}^{149} \mathrm{Sm},{ }_{63}^{151} \mathrm{Eu},{ }_{64}^{155} \mathrm{Gd}$ and ${ }_{64}^{155} \mathrm{Gd}$ are found in very small quantities in the reactor.

From the isotopic abundances of the yields, one can extract information about the nuclear reactions at the time the reactor was operational and reconstruct the reaction rates at that time. One of the key quantity measured is the ratio ${ }_{62}^{149} \mathrm{Sm} /{ }_{62}^{147} \mathrm{Sm}$ of two light isotopes of samarium, which are not fission products. This ratio of order of 0.9 in normal samarium, is about 0.02 in Oklo ores. This low value is interpreted [465] by the depletion of ${ }_{62}^{149} \mathrm{Sm}$ by thermal neutrons produced by the fission process and to which it was exposed while the reactor was active. The capture cross section of thermal neutron by ${ }_{62}^{149} \mathrm{Sm}$

$$
n+{ }_{62}^{149} \mathrm{Sm} \longrightarrow{ }_{62}^{150} \mathrm{Sm}+\gamma
$$

is dominated by a capture resonance of a neutron of energy of about $0.1 \mathrm{eV}\left(E_{r}=97.3 \mathrm{meV}\right.$ today). The existence of this resonance is a consequence of an almost cancellation between the electromagnetic repulsive force and the strong interaction.

Shlyakhter [465] pointed out that this phenomenon can be used to set a constraint on the time variation of fundamental constants. His argument can be summarized as follows.

- First, the cross section $\sigma_{(n, \gamma)}$ strongly depends on the energy of a resonance at $E_{r}=$ $97.3 \mathrm{meV}$.

- Geochemical data allow to determine the isotopic composition of various element, such as uranium, neodynium, gadolinium and samarium. Gadolinium and neodium allow to determine the fluence (integrated flux over time) of the neutron while both gadolinium and samarium are strong neutron absorbers.

- From these data, one deduces the value of the averaged value of the cross section on the neutron flux, $\hat{\sigma}_{149}$. This value depends on hypothesis on the geometry of the reactor zone.

- The range of allowed value of $\hat{\sigma}_{149}$ was translated into a constraint on $E_{r}$. This step involves an assumption on the form and temperature of the neutron spectrum.

- $E_{r}$ was related to some fundamental constant, which involve a model of the nucleus.

In conclusion, we have different steps, which all involve assumptions:

- Isotopic compositions and geophysical parameters are measured in a given set of bore-hold in each zone. A choice has to be made on the sample to use, in order, e.g., to ensure that they are not contaminated.

- With hypothesis on the geometry of the reactor, on the spectrum and temperature of the neutron flux, one can deduce the effective value of the cross sections of neutron absorbers (such as samarium and gadolinium). This requires one to solve a network of nuclear reactions describing the fission.

- One can then infer the value of the resonance energy $E_{r}$, which again depends on the assumptions on the neutron spectrum. 
- $E_{r}$ needs to be related to fundamental constant, which involves a model of the nucleus and high energy physics hypothesis.

We shall now detail the assumptions used in the various analyses that have been performed since the pioneering work of [465].

\subsubsection{Constraining the shift of the resonance energy}

Cross sections. The cross section of the neutron capture (38) strongly depends on the energy of a resonance at $E_{r}=97.3 \mathrm{meV}$ and is well described by the Breit-Wigner formula

$$
\sigma_{(n, \gamma)}(E)=\frac{g_{0} \pi}{2} \frac{\hbar^{2}}{m_{\mathrm{n}} E} \frac{\Gamma_{\mathrm{n}} \Gamma_{\gamma}}{\left(E-E_{r}\right)^{2}+\Gamma^{2} / 4}
$$

where $g_{0} \equiv(2 J+1)(2 s+1)^{-1}(2 I+1)^{-1}$ is a statistical factor, which depends on the spin of the incident neutron $s=1 / 2$, of the target nucleus $I$, and of the compound nucleus $J$. For the reaction (38), we have $g_{0}=9 / 16$. The total width $\Gamma \equiv \Gamma_{\mathrm{n}}+\Gamma_{\gamma}$ is the sum of the neutron partial width $\Gamma_{\mathrm{n}}=0.533 \mathrm{meV}$ (at $E_{r}=97.3 \mathrm{meV}$ and it scales as $\sqrt{E}$ in the center of mass) and of the radiative partial width $\Gamma_{\gamma}=60.5 \mathrm{meV} .{ }_{64}^{155} \mathrm{Gd}$ has a resonance at $E_{r}=26.8 \mathrm{meV}$ with $\Gamma_{\mathrm{n}}=0.104 \mathrm{meV}, \Gamma_{\gamma}=108 \mathrm{meV}$ and $g=5 / 8$ while ${ }_{64}^{157} \mathrm{Gd}$ has a resonance at $E_{r}=31.4 \mathrm{meV}$ with $\Gamma_{\mathrm{n}}=0.470 \mathrm{meV}, \Gamma_{\gamma}=106 \mathrm{meV}$ and $g=5 / 8$.

As explained in the previous Section 3.2.1, this cross section cannot be measured from the Oklo data, which allow only to measure its value averaged on the neutron flux $n(v, T), T$ being the temperature of the moderator. It is conventionally defined as

$$
\hat{\sigma}=\frac{1}{n v_{0}} \int \sigma_{(n, \gamma)} n(v, T) v \mathrm{~d} v
$$

where the velocity $v_{0}=2200 \mathrm{~m} \cdot \mathrm{s}^{-1}$ corresponds to an energy $E_{0}=25.3 \mathrm{meV}$ and $v=\sqrt{2 E / m_{\mathrm{n}}}$, instead of

$$
\bar{\sigma}=\frac{\int \sigma_{(n, \gamma)} n(v, T) v \mathrm{~d} v}{\int n(v, T) v \mathrm{~d} v} .
$$

When the cross section behaves as $\sigma=\sigma_{0} v_{0} / v$, which is the case for nuclei known as " $1 / v$ absorbers", $\hat{\sigma}=\sigma_{0}$ and does not depend on the temperature, whatever the distribution $n(v)$. In a similar way, the effective neutron flux defined

$$
\hat{\phi}=v_{0} \int n(v, T) \mathrm{d} v
$$

which differs from the true flux

$$
\phi=\int n(v, T) v \mathrm{~d} v .
$$

However, since $\bar{\sigma} \phi=\hat{\sigma} \hat{\phi}$, the reaction rates are not affected by these definitions.

Extracting the effective cross section from the data. To "measure" the value of $\hat{\sigma}$ from the Oklo data, we need to solve the nuclear reaction network that controls the isotopic composition during the fission.

The samples of the Oklo reactors were exposed [382] to an integrated effective fluence $\int \hat{\phi} \mathrm{d} t$ of about $10^{21}$ neutron $\cdot \mathrm{cm}^{-2}=1 \mathrm{~kb}^{-1}$. It implies that any process with a cross section smaller than $1 \mathrm{~kb}$ can safely be neglected in the computation of the abundances. This includes neutron capture by ${ }_{62}^{144} \mathrm{Sm}$ and ${ }_{62}^{148} \mathrm{Sm}$, as well as by ${ }_{64}^{155} \mathrm{Gd}$ and ${ }_{64}^{157} \mathrm{Gd}$. On the other hand, the fission of ${ }_{92}^{235} \mathrm{U}$, the 
Table 8: Summary of the analysis of the Oklo data. The principal assumptions to infer the value of the resonance energy $E_{r}$ are the form of the neutron spectrum and its temperature.

\begin{tabular}{lccccc}
\hline Ore & neutron spectrum & Temperature $\left({ }^{\circ} \mathrm{C}\right)$ & $\hat{\sigma}_{149}(\mathrm{~kb})$ & $\Delta E_{r}(\mathrm{meV})$ & Ref. \\
\hline$?$ & Maxwell & 20 & $55 \pm 8$ & $0 \pm 20$ & {$[465]$} \\
RZ2 $(15)$ & Maxwell & $180-700$ & $75 \pm 18$ & $-1.5 \pm 10.5$ & {$[123]$} \\
RZ10 & Maxwell & $200-400$ & $91 \pm 6$ & $4 \pm 16$ & {$[220]$} \\
RZ10 & & & & $-97 \pm 8$ & {$[220]$} \\
- & Maxwell + epithermal & 327 & $91 \pm 6$ & $-45_{-15}^{+7}$ & {$[305]$} \\
RZ2 & Maxwell + epithermal & & $73.2 \pm 9.4$ & $-5.5 \pm 67.5$ & {$[416]$} \\
RZ2 & Maxwell + epithermal & $200-300$ & $71.5 \pm 10.0$ & - & {$[234]$} \\
RZ10 & Maxwell + epithermal & $200-300$ & $85.0 \pm 6.8$ & - & {$[234]$} \\
RZ2+RZ10 & & & & $7.2 \pm 18.8$ & {$[234]$} \\
RZ2+RZ10 & & & & $90.75 \pm 11.15$ & {$[234]$} \\
\hline
\end{tabular}

capture of neutron by ${ }_{60}^{143} \mathrm{Nd}$ and by ${ }_{62}^{149} \mathrm{Sm}$ with respective cross sections $\sigma_{5} \simeq 0.6 \mathrm{~kb}, \sigma_{143} \sim 0.3 \mathrm{~kb}$ and $\sigma_{149} \geq 70 \mathrm{~kb}$ are the dominant processes. It follows that the equations of evolution for the number densities $N_{147}, N_{148}, N_{149}$ and $N_{235}$ of ${ }_{62}^{147} \mathrm{Sm},{ }_{62}^{148} \mathrm{Sm},{ }_{62}^{149} \mathrm{Sm}$ and ${ }_{92}^{235} \mathrm{U}$ takes the form

$$
\begin{aligned}
& \frac{\mathrm{d} N_{147}}{\hat{\phi} \mathrm{d} t}=-\hat{\sigma}_{147} N_{147}+\hat{\sigma}_{f 235} y_{147} N_{235} \\
& \frac{\mathrm{d} N_{148}}{\hat{\phi} \mathrm{d} t}=\hat{\sigma}_{147} N_{147} \\
& \frac{\mathrm{d} N_{149}}{\hat{\phi} \mathrm{d} t}=-\hat{\sigma}_{149} N_{149}+\hat{\sigma}_{f 235} y_{149} N_{235} \\
& \frac{\mathrm{d} N_{235}}{\hat{\phi} \mathrm{d} t}=-\sigma_{5} N_{235}
\end{aligned}
$$

where $y_{i}$ denotes the yield of the corresponding element in the fission of ${ }_{92}^{235} \mathrm{U}$ and $\hat{\sigma}_{5}$ is the fission cross section. This system can be integrated under the assumption that the cross sections and the neutron flux are constant and the result compared with the natural abundances of the samarium to extract the value of $\hat{\sigma}_{149}$ at the time of the reaction. Here, the system has been closed by introducing a modified absorption cross section [123] $\sigma_{5}^{*}$ to take into account both the fission, capture but also the formation from the $\alpha$-decay of ${ }_{94}^{239} \mathrm{Pu}$. One can instead extend the system by considering ${ }_{94}^{239} \mathrm{Pu}$, and ${ }_{92}^{235} \mathrm{U}$ (see [234]). While most studies focus on the samarium, [220] also includes the gadolinium even though it is not clear whether it can reliably be measured [123]. They give similar results.

By comparing the solution of this system with the measured isotopic composition, one can deduce the effective cross section. At this step, the different analyses [465, 415, 123, 220, 305, 416, $234]$ differ from the choice of the data. The measured values of $\hat{\sigma}_{149}$ can be found in these articles. They are given for a given zone (RZ2, RZ10 mainly) with a number that correspond to the number of the bore-hole and the depth (e.g., in Table 2 of [123], SC39-1383 means that we are dealing with the bore-hole number 39 at a depth of $13.83 \mathrm{~m}$ ). Recently, another approach [416, 234] was proposed in order to take into account of the geometry and details of the reactor. It relies on a full-scale Monte-Carlo simulation and a computer model of the reactor zone RZ2 [416] and both RZ2 and RZ10 [234] and allows to take into account the spatial distribution of the neutron flux. 
Determination of $\boldsymbol{E}_{\boldsymbol{r}}$. To convert the constraint on the effective cross section, one needs to specify the neutron spectrum. In the earlier studies [465, 415], a Maxwell distribution,

$$
n_{\mathrm{th}}(v, T)=\left(\frac{m_{\mathrm{n}}}{2 \pi T}\right)^{3 / 2} \mathrm{e}^{-\frac{m v^{2}}{2 k_{\mathrm{B}} T}}
$$

was assumed for the neutron with a temperature of $20^{\circ} \mathrm{C}$, which is probably too small. Then $v_{0}$ is the mean velocity at a temperature $T_{0}=m_{\mathrm{n}} v_{0}^{2} / 2 k_{\mathrm{B}}=20.4^{\circ} \mathrm{C}$. [123, 220] also assume a Maxwell distribution but let the moderator temperature vary so that they deduce an effective cross section $\hat{\sigma}\left(R_{r}, T\right)$. They respectively restricted the temperature range to $180^{\circ} \mathrm{C}<T<700^{\circ} \mathrm{C}$ and $200^{\circ} \mathrm{C}<T<400^{\circ} \mathrm{C}$, based on geochemical analysis. The advantage of the Maxwell distribution assumption is that it avoids to rely on a particular model of the Oklo reactor since the spectrum is determined solely by the temperature.

It was then noted $[305,416]$ that above an energy of several eV, the neutron spectrum shifted to a $1 / E$ tail because of the absorption of neutrons in uranium resonances. Thus, the distribution was adjusted to include an epithermal distribution

$$
n(v)=(1-f) n_{\mathrm{th}}(v, T)+f n_{\mathrm{epi}}(v),
$$

with $n_{\text {epi }}=v_{c}^{2} / v^{2}$ for $v>v_{c}$ and vanishing otherwise. $v_{c}$ is a cut-off velocity that also needs to be specified. The effective cross section can then be parameterized [234] as

$$
\hat{\sigma}=g(T) \sigma_{0}+r_{0} I
$$

where $g(T)$ is a measure of the departure of $\sigma$ from the $1 / v$ behavior, $I$ is related to the resonance integral of the cross section and $r_{0}$ is the Oklo reactor spectral index. It characterizes the contribution of the epithermal neutrons to the cross section. Among the unknown parameters, the most uncertain is probably the amount of water present at the time of the reaction. [234] chooses to adjust it so that $r_{0}$ matches the experimental values.

These hypothesis on the neutron spectrum and on the temperature, as well as the constraint on the shift of the resonance energy, are summarized in Table 8. Many analyses [220, 416, 234] find two branches for $\Delta E_{r}=E_{r}-E_{r 0}$, with one (the left branch) indicating a variation of $E_{r}$. Note that these two branches disappear when the temperature is higher since $\hat{\sigma}\left(E_{r}, T\right)$ is more peaked when $T$ decreases but remain in any analysis at low temperature. This shows the importance of a good determination of the temperature. Note that the analysis of [416] indicates that the curves $\hat{\sigma}\left(T, E_{r}\right)$ lie appreciably lower than for a Maxwell distribution and that [220] argues that the left branch is hardly compatible with the gadolinium data.

\subsubsection{From the resonance energy to fundamental constants}

The energy of the resonance depends a priori on many constants since the existence of such resonance is mainly the consequence of an almost cancellation between the electromagnetic repulsive force and the strong interaction. But, since no full analytical understanding of the energy levels of heavy nuclei is available, the role of each constant is difficult to disentangle.

In his first analysis, Shlyakhter [465] stated that for the neutron, the nucleus appears as a potential well with a depth $V_{0} \simeq 50 \mathrm{MeV}$. He attributed the change of the resonance energy to a modification of the strong interaction coupling constant and concluded that $\Delta g_{\mathrm{S}} / g_{\mathrm{S}} \sim \Delta E_{r} / V_{0}$. Then, arguing that the Coulomb force increases the average inter-nuclear distance by about $2.5 \%$ for $A \sim 150$, he concluded that $\Delta \alpha_{\mathrm{EM}} / \alpha_{\mathrm{EM}} \sim 20 \Delta g_{\mathrm{S}} / g_{\mathrm{S}}$, leading to $\left|\alpha_{\mathrm{EM}} / \alpha_{\mathrm{EM}}\right|<10^{-17} \mathrm{yr}^{-1}$, which can be translated to

$$
\left|\Delta \alpha_{\mathrm{EM}} / \alpha_{\mathrm{EM}}\right|<1.8 \times 10^{-8}
$$

Living Reviews in Relativity

http: //www . livingreviews . org/lrr-2011-2 
The following analysis focused on the fine-structure constant and ignored the strong interaction. Damour and Dyson [123] related the variation of $E_{r}$ to the fine-structure constant by taking into account that the radiative capture of the neutron by ${ }_{62}^{149} \mathrm{Sm}$ corresponds to the existence of an excited quantum state of ${ }_{62}^{150} \mathrm{Sm}$ (so that $E_{r}=E_{150}^{*}-E_{149}-m_{\mathrm{n}}$ ) and by assuming that the nuclear energy is independent of $\alpha_{\mathrm{EM}}$. It follows that the variation of $\alpha_{\mathrm{EM}}$ can be related to the difference of the Coulomb binding energy of these two states. The computation of this latter quantity is difficult and must be related to the mean-square radii of the protons in the isotopes of samarium. In particular this analysis [123] showed that the Bethe-Weizäcker formula overestimates by about a factor the 2 the $\alpha_{\mathrm{EM}}$-sensitivity to the resonance energy. It follows from this analysis that

$$
\alpha_{\mathrm{EM}} \frac{\Delta E_{r}}{\Delta \alpha_{\mathrm{EM}}} \simeq-1.1 \mathrm{MeV},
$$

which, once combined with the constraint on $\Delta E_{r}$, implies

$$
-0.9 \times 10^{-7}<\Delta \alpha_{\mathrm{EM}} / \alpha_{\mathrm{EM}}<1.2 \times 10^{-7}
$$

at $2 \sigma$ level, corresponding to the range $-6.7 \times 10^{-17} \mathrm{yr}^{-1}<\alpha_{\mathrm{EM}} / \alpha_{\mathrm{EM}}<5.0 \times 10^{-17} \mathrm{yr}^{-1}$ if $\alpha_{\mathrm{EM}}$ is assumed constant. This tight constraint arises from the large amplification between the resonance energy $(\sim 0.1 \mathrm{eV})$ and the sensitivity $(\sim 1 \mathrm{MeV})$. The re-analysis of these data and also including the data of [220] with gadolinium, found the favored result $\alpha_{\mathrm{EM}} / \alpha_{\mathrm{EM}}=(-0.2 \pm 0.8) \times 10^{-17} \mathrm{yr}^{-1}$, which corresponds to

$$
\Delta \alpha_{\mathrm{EM}} / \alpha_{\mathrm{EM}}=(-0.36 \pm 1.44) \times 10^{-8}
$$

and the other branch (indicating a variation; see Table 8) leads to $\alpha_{\mathrm{EM}} / \alpha_{\mathrm{EM}}=(4.9 \pm 0.4) \times$ $10^{-17} \mathrm{yr}^{-1}$. This non-zero result cannot be eliminated.

The more recent analysis, based on a modification of the neutron spectrum lead respectively to $[416]$

and [234]

$$
\Delta \alpha_{\mathrm{EM}} / \alpha_{\mathrm{EM}}=(3.85 \pm 5.65) \times 10^{-8}
$$

$$
\Delta \alpha_{\mathrm{EM}} / \alpha_{\mathrm{EM}}=(-0.65 \pm 1.75) \times 10^{-8},
$$

at a $95 \%$ confidence level, both using the formalism of [123].

Olive et al. [399], inspired by grand unification model, reconsider the analysis of [123] by letting all gauge and Yukawa couplings vary. Working within the Fermi gas model, the over-riding scale dependence of the terms, which determine the binding energy of the heavy nuclei was derived. Parameterizing the mass of the hadrons as $m_{i} \propto \Lambda_{\mathrm{QCD}}\left(1+\kappa_{i} m_{\mathrm{q}} / \Lambda_{\mathrm{QCD}}+\ldots\right)$, they estimate that the nuclear Hamiltonian was proportional to $m_{\mathrm{q}} / \Lambda_{\mathrm{QCD}}$ at lowest order, which allows to estimate that the energy of the resonance is related to the quark mass by

$$
\frac{\Delta E_{r}}{E_{r}} \sim(2.5-10) \times 10^{17} \Delta \ln \left(\frac{m_{\mathrm{q}}}{\Lambda_{\mathrm{QCD}}}\right) .
$$

Using the constraint (48), they first deduced that

$$
\left|\Delta \ln \left(\frac{m_{\mathrm{q}}}{\Lambda_{\mathrm{QCD}}}\right)\right|<(1-4) \times 10^{-8} .
$$

Then, assuming that $\alpha_{\mathrm{EM}} \propto m_{\mathrm{q}}^{50}$ on the basis of grand unification (see Section 6.3 for details), they concluded that

$$
\left|\Delta \alpha_{\mathrm{EM}} / \alpha_{\mathrm{EM}}\right|<(2-8) \times 10^{-10} .
$$

Similarly, $[207,467,212]$ related the variation of the resonance energy to the quark mass. Their first estimate [207] assumes that it is related to the pion mass, $m_{\pi}$, and that the main variation 
arises from the variation of the radius $R \sim 5 \mathrm{fm}+1 / m_{\pi}$ of the nuclear potential well of depth $V_{0}$, so that

$$
\delta E_{r} \sim-2 V_{0} \frac{\delta R}{R} \sim 3 \times 10^{8} \frac{\delta m_{\pi}}{m_{\pi}}
$$

assuming that $R \simeq 1.2 A^{1 / 3} r_{0}, r_{0}$ being the inter-nucleon distance.

Then, in [467], the nuclear potential was described by a Walecka model, which keeps only the $\sigma$ (scalar) and $\omega$ (vector) exchanges in the effective nuclear force. Their masses was related to the mass $m_{\mathrm{s}}$ of the strange quark to get $m_{\sigma} \propto m_{\mathrm{s}}^{0.54}$ and $m_{\omega} \propto m_{\mathrm{s}}^{0.15}$. It follows that the variation of the potential well can be related to the variation of $m_{\sigma}$ and $m_{\omega}$ and thus on $m_{\mathrm{q}}$ by $V \propto m_{\mathrm{q}}^{-3.5}$. The constraint (48) then implies that

$$
\left|\Delta \ln \left(\frac{m_{\mathrm{s}}}{\Lambda_{\mathrm{QCD}}}\right)\right|<1.2 \times 10^{-10} .
$$

By extrapolating from light nuclei where the $N$-body calculations can be performed more accurately, it was concluded [208] that the resonance energy scales as $\Delta E_{r} \simeq 10\left(\Delta \ln X_{\mathrm{q}}-0.1 \Delta \ln \alpha_{\mathrm{EM}}\right)$, so that the the constraints from [416] would imply that $\Delta \ln \left(X_{\mathrm{q}} / \alpha_{\mathrm{EM}}^{0.1}\right)<7 \times 10^{-9}$.

In conclusion, these last results illustrate that a detailed theoretical analysis and quantitative estimates of the nuclear physics (and QCD) aspects of the resonance shift still remain to be carried out. In particular, the interface between the perturbative QCD description and the description in term of hadron is not fully understand: we do not know the exact dependence of hadronic masses and coupling constant on $\Lambda_{\mathrm{QCD}}$ and quark masses. The second problem concerns modeling nuclear forces in terms of the hadronic parameters.

At present, the Oklo data, while being stringent and consistent with no variation, have to be considered carefully. While a better understanding of nuclear physics is necessary to understand the full constant-dependence, the data themselves require more insight, particularly to understand the existence of the left-branch.

\subsection{Meteorite dating}

Long-lived $\alpha$ - or $\beta$-decay isotopes may be sensitive probes of the variation of fundamental constants on geological times ranging typically to the age of the solar system, $t \sim(4-5)$ Gyr, corresponding to a mean redshift of $z \sim 0.43$. Interestingly, it can be compared with the shallow universe quasar constraints. This method was initially pointed out by Wilkinson [539] and then revived by Dyson [168]. The main idea is to extract the $\alpha_{\mathrm{EM}}$-dependence of the decay rate and to use geological samples to bound its time variation.

The sensitivity of the decay rate of a nucleus to a change of the fine-structure constant is defined, in a similar way as for atomic clocks [Equation (23)], as

$$
s_{\alpha} \equiv \frac{\partial \ln \lambda}{\partial \ln \alpha_{\mathrm{EM}}} .
$$

$\lambda$ is a function of the decay energy $Q$. When $Q$ is small, mainly due to an accidental cancellation between different contributions to the nuclear binding energy, the sensitivity $s_{\alpha}$ maybe strongly enhanced. A small variation of the fundamental constants can either stabilize or destabilize certain isotopes so that one can extract bounds on the time variation of their lifetime by comparing laboratory data to geophysical and solar system probes.

Assume some meteorites containing an isotope $X$ that decays into $Y$ are formed at a time $t_{*}$. It follows that

$$
N_{X}(t)=N_{X *} \mathrm{e}^{-\lambda\left(t-t_{*}\right)}, \quad N_{Y}(t)=N_{X *}\left[1-\mathrm{e}^{-\lambda\left(t-t_{*}\right)}\right]+N_{Y *}
$$


if one assumes the decay rate constant. If it is varying then these relations have to be replaced by

$$
N_{X}(t)=N_{X *} \mathrm{e}^{\int_{t_{*}}^{t} \lambda\left(t^{\prime}\right) \mathrm{d} t^{\prime}}
$$

so that the value of $N_{X}$ today can be interpreted with Equation (56) but with an effective decay rate of

$$
\bar{\lambda}=\frac{1}{t_{0}-t_{*}} \int_{t_{*}}^{t_{0}} \lambda\left(t^{\prime}\right) \mathrm{d} t^{\prime}
$$

From a sample of meteorites, we can measure $\left\{N_{X}\left(t_{0}\right), N_{Y}\left(t_{0}\right)\right\}$ for each meteorite. These two quantities are related by

$$
N_{Y}\left(t_{0}\right)=\left[\mathrm{e}^{\bar{\lambda}\left(t_{0}-t_{*}\right)}-1\right] N_{X}\left(t_{0}\right)+N_{Y *},
$$

so that the data should lie on a line (since $N_{X *}$ is a priori different for each meteorite), called an "isochron", the slope of which determines $\bar{\lambda}\left(t_{0}-t_{*}\right)$. It follows that meteorites data only provides an average measure of the decay rate, which complicates the interpretation of the constraints (see $[219,218]$ for explicit examples). To derive a bound on the variation of the constant we also need a good estimation of $t_{0}-t_{*}$, which can be obtained from the same analysis for an isotope with a small sensitivity $s_{\alpha}$, as well as an accurate laboratory measurement of the decay rate.

\subsubsection{Long lived $\alpha$-decays}

The $\alpha$-decay rate, $\lambda$, of a nucleus ${ }_{Z}^{A} \mathrm{X}$ of charge $Z$ and atomic number $A$,

$$
{ }_{Z+2}^{A+4} \mathrm{X} \longrightarrow{ }_{Z}^{A} \mathrm{X}+{ }_{2}^{4} \mathrm{He}
$$

is governed by the penetration of the Coulomb barrier that can be described by the Gamow theory. It is well approximated by

$$
\lambda \simeq \Lambda\left(\alpha_{\mathrm{EM}}, v\right) \exp \left(-4 \pi Z \alpha_{\mathrm{EM}} \frac{c}{v}\right)
$$

where $v / c=\sqrt{Q / 2 m_{\mathrm{p}} c^{2}}$ is the escape velocity of the $\alpha$ particle. $\Lambda$ is a function that depends slowly on $\alpha_{\mathrm{EM}}$ and $Q$. It follows that the sensitivity to the fine-structure constant is

$$
s_{\alpha} \simeq-4 \pi Z \frac{\alpha_{\mathrm{EM}}}{\sqrt{Q / 2 m_{\mathrm{p}}}}\left(1-\frac{1}{2} \frac{\mathrm{d} \ln Q}{\mathrm{~d} \ln \alpha_{\mathrm{EM}}}\right) .
$$

The decay energy is related to the nuclear binding energies $B(A, Z)$ of the different nuclei by

$$
Q=B(A, Z)+B_{\alpha}-B(A+4, Z+2)
$$

with $B_{\alpha}=B(4,2)$. Physically, an increase of $\alpha_{\mathrm{EM}}$ induces an increase in the height of the Coulomb barrier at the nuclear surface while the depth of the nuclear potential well below the top remains the same. It follows that $\alpha$-particle escapes with a greater energy but at the same energy below the top of the barrier. Since the barrier becomes thiner at a given energy below its top, the penetrability increases. This computation indeed neglects the effect of a variation of $\alpha_{\mathrm{EM}}$ on the nucleus that can be estimated to be dilated by about $1 \%$ if $\alpha_{\mathrm{EM}}$ increases by $1 \%$.

As a first insight, when focusing on the fine-structure constant, one can estimate $s_{\alpha}$ by varying only the Coulomb term of the binding energy. Its order of magnitude can be estimated from the Bethe-Weizäcker formula

$$
E_{\mathrm{EM}}=98.25 \frac{Z(Z-1)}{A^{1 / 3}} \alpha_{\mathrm{EM}} \mathrm{MeV}
$$


Table 9: Summary of the main nuclei and their physical properties that have been used in $\alpha$-decay studies.

\begin{tabular}{lccccc}
\hline Element & $Z$ & $A$ & Lifetime $(\mathrm{yr})$ & $Q(\mathrm{MeV})$ & $s_{\alpha}$ \\
\hline Sm & 62 & 147 & $1.06 \times 10^{11}$ & 2.310 & 774 \\
$\mathrm{Gd}$ & 64 & 152 & $1.08 \times 10^{14}$ & 2.204 & 890 \\
$\mathrm{Dy}$ & 66 & 154 & $3 \times 10^{6}$ & 2.947 & 575 \\
$\mathrm{Pt}$ & 78 & 190 & $6.5 \times 10^{11}$ & 3.249 & 659 \\
$\mathrm{Th}$ & 90 & 232 & $1.41 \times 10^{10}$ & 4.082 & 571 \\
$\mathrm{U}$ & 92 & 235 & $7.04 \times 10^{8}$ & 4.678 & 466 \\
$\mathrm{U}$ & 92 & 238 & $4.47 \times 10^{9}$ & 4.270 & 548 \\
\hline
\end{tabular}

Table 9 summarizes the most sensitive isotopes, with the sensitivities derived from a semiempirical analysis for a spherical nucleus [399]. They are in good agreement with the ones derived from Equation (61) (e.g., for ${ }^{238} \mathrm{U}$, one would obtain $s_{\alpha}=540$ instead of $s_{\alpha}=548$ ).

The sensitivities of all the nuclei of Table 9 are similar, so that the best constraint on the time variation of the fine-structure constant will be given by the nuclei with the smaller $\Delta \lambda / \lambda$.

Wilkinson [539] considered the most favorable case, that is the decay of ${ }_{92}^{238} \mathrm{U}$ for which $s_{\alpha}=548$ (see Table 9). By comparing the geological dating of the Earth by different methods, he concluded that the decay constant $\lambda$ of ${ }^{238} \mathrm{U},{ }^{235} \mathrm{U}$ and ${ }^{232}$ Th have not changed by more than a factor 3 or 4 during the last $3-4 \times 10^{9}$ years from which it follows

$$
\left|\Delta \alpha_{\mathrm{EM}} / \alpha_{\mathrm{EM}}\right|<8 \times 10^{-3}
$$

This constraint was revised by Dyson [168] who claimed that the decay rate has not changed by more than $20 \%$, during the past $2 \times 10^{9}$ years, which implies

$$
\left|\Delta \alpha_{\mathrm{EM}} / \alpha_{\mathrm{EM}}\right|<4 \times 10^{-4} .
$$

Uranium has a short lifetime so that it cannot be used to set constraints on longer time scales. It is also used to calibrate the age of the meteorites. Therefore, it was suggested [399] to consider ${ }^{147} \mathrm{Sm}$. Assuming that $\Delta \lambda_{147} / \lambda_{147}$ is smaller than the fractional uncertainty of $7.5 \times 10^{-3}$ of its half-life

$$
\left|\Delta \alpha_{\mathrm{EM}} / \alpha_{\mathrm{EM}}\right| \lesssim \times 10^{-5}
$$

As for the Oklo phenomena, the effect of other constants has not been investigated in depth. It is clear that at lowest order both $Q$ and $m_{\mathrm{p}}$ scales as $\Lambda_{\mathrm{QCD}}$ so that one needs to go beyond such a simple description to determine the dependence in the quark masses. Taking into account the contribution of the quark masses, in the same way as for Equation (53), it was argued that $\lambda \propto X_{\mathrm{q}}^{300-2000}$, which leads to $\left|\Delta \ln X_{\mathrm{q}}\right| \lesssim 10^{-5}$. In a grand unify framework, that could lead to a constraint of the order of $\left|\Delta \ln \alpha_{\mathrm{EM}}\right| \lesssim 2 \times 10^{-7}$.

\subsubsection{Long lived $\beta$-decays}

Dicke [150] stressed that the comparison of the rubidium-strontium and potassium-argon dating methods to uranium and thorium rates constrains the variation of $\alpha_{\mathrm{EM}}$.

As long as long-lived $\beta$-decay isotopes are concerned for which the decay energy $Q$ is small, we can use a non-relativistic approximation for the decay rate

$$
\lambda=\Lambda_{ \pm} Q^{p_{ \pm}}
$$

respectively for $\beta^{-}$-decay and electron capture. $\Lambda_{ \pm}$are functions that depend smoothly on $\alpha_{\mathrm{EM}}$ and which can thus be considered constant, $p_{+}=\ell+3$ and $p_{-}=2 \ell+2$ are the degrees of 
forbiddenness of the transition. For high- $Z$ nuclei with small decay energy $Q$, the exponent $p$ becomes $p=2+\sqrt{1-\alpha_{\mathrm{EM}}^{2} Z^{2}}$ and is independent of $\ell$. It follows that the sensitivity to a variation of the fine-structure constant is

$$
s_{\alpha}=p \frac{\mathrm{d} \ln Q}{\mathrm{~d} \ln \alpha_{\mathrm{EM}}} .
$$

The second factor can be estimated exactly as for $\alpha$-decay. We note that $\Lambda_{ \pm}$depends on the Fermi constant and on the mass of the electron as $\Lambda_{ \pm} \propto G_{\mathrm{F}}^{2} m_{\mathrm{e}}^{5} Q^{p}$. This dependence is the same for any $\beta$-decay so that it will disappear in the comparison of two dating methods relying on two different $\beta$-decay isotopes, in which case only the dependence on the other constants appear again through the nuclear binding energy. Note, however, that comparing a $\alpha$ - to a $\beta$-decay may lead to interesting constraints.

We refer to Section III.A.4 of FVC [500] for earlier constraints derived from rubidium-strontium, potassium-argon and we focus on the rhenium-osmium case,

$$
{ }_{75}^{187} \mathrm{Re} \longrightarrow{ }_{76}^{187} \mathrm{Os}+\bar{\nu}_{e}+e^{-}
$$

first considered by Peebles and Dicke [406]. They noted that the very small value of its decay energy $Q=2.6 \mathrm{keV}$ makes it a very sensitive probe of the variation of $\alpha_{\mathrm{EM}}$. In that case $p \simeq 2.8$ so that $s_{\alpha} \simeq-18000$; a change of $10^{-2 \%}$ of $\alpha_{\mathrm{EM}}$ will induce a change in the decay energy of order of the keV, that is of the order of the decay energy itself. Peebles and Dicke [406] did not have reliable laboratory determination of the decay rate to put any constraint. Dyson [167] compared the isotopic analysis of molybdenite ores $\left(\lambda_{187}=(1.6 \pm 0.2) \times 10^{-11} \mathrm{yr}^{-1}\right)$, the isotopic analysis of 14 iron meteorites $\left(\lambda_{187}=(1.4 \pm 0.3) \times 10^{-11} \mathrm{yr}^{-1}\right)$ and laboratory measurements of the decay rate $\left(\lambda_{187}=(1.1 \pm 0.1) \times 10^{-11} \mathrm{yr}^{-1}\right)$. Assuming that the variation of the decay energy comes entirely from the variation of $\alpha_{\mathrm{EM}}$, he concluded that $\left|\Delta \alpha_{\mathrm{EM}} / \alpha_{\mathrm{EM}}\right|<9 \times 10^{-4}$ during the past $3 \times 10^{9}$ years. Note that the discrepancy between meteorite and lab data could have been interpreted as a time-variation of $\alpha_{\mathrm{EM}}$, but the laboratory measurement were complicated by many technical issues so that Dyson only considered a conservative upper limit.

The modelization and the computation of $s_{\alpha}$ were improved in [399], following the same lines as for $\alpha$-decay.

$$
\frac{\Delta \lambda_{187}}{\lambda_{187}}=p \frac{\Delta Q}{Q} \simeq p\left(\frac{20 \mathrm{MeV}}{Q}\right) \frac{\Delta \alpha_{\mathrm{EM}}}{\alpha_{\mathrm{EM}}} \sim-2.2 \times 10^{4} \frac{\Delta \alpha_{\mathrm{EM}}}{\alpha_{\mathrm{EM}}}
$$

if one considers only the variation of the Coulomb energy in $Q$. A similar analysis [147] leads to $\Delta \ln \lambda_{187} \simeq 10^{4} \Delta \ln \left[\alpha_{\mathrm{EM}}^{-2.2} X_{\mathrm{q}}^{-1.9}\left(X_{\mathrm{d}}-X_{\mathrm{u}}\right)^{0.23} X_{\mathrm{e}}^{-0.058}\right]$.

The dramatic improvement in the meteoric analysis of the Re/Os ratio [468] led to a recent reanalysis of the constraints on the fundamental constants. The slope of the isochron was determined with a precision of $0.5 \%$. However, the Re/Os ratio is inferred from iron meteorites the age of which is not determined directly. Models of formation of the solar system tend to show that iron meteorites and angrite meteorites form within the same 5 million years. The age of the latter can be estimated from the ${ }^{207} \mathrm{~Pb}^{208} \mathrm{~Pb}$ method, which gives $4.558 \mathrm{Gyr}$ [337] so that $\lambda_{187}=$ $(1.666 \pm 0.009) \times 10^{-11} \mathrm{yr}^{-1}$. Thus, we could adopt [399]

$$
\left|\frac{\Delta \lambda_{187}}{\lambda_{187}}\right|<5 \times 10^{-3}
$$

However, the meteoritic ages are determined mainly by ${ }^{238} \mathrm{U}$ dating so that effectively we have a constraint on the variation of $\lambda_{187} / \lambda_{238}$. Fortunately, since the sensitivity of ${ }^{238} \mathrm{U}$ is much smaller than the one of the rhenium, it is safe to neglect its effect. Using the recent laboratory measurement [333] $\left(\lambda_{187}=(-1.639 \pm 0.025) \times 10^{-11} \mathrm{yr}^{-1}\right)$, the variation of the decay rate is not 
given by the dispersion of the meteoritic measurement, but by comparing to its value today, so that

$$
\left|\frac{\Delta \lambda_{187}}{\lambda_{187}}\right|=-0.016 \pm 0.016 .
$$

The analysis of Ref. [400], following the assumption of [399], deduced that

$$
\Delta \alpha_{\mathrm{EM}} / \alpha_{\mathrm{EM}}=(-8 \pm 16) \times 10^{-7},
$$

at a $95 \%$ confidence level, on a typical time scale of 5 Gyr (or equivalently a redshift of order $z \sim 0.2)$.

As pointed out in $[219,218]$, these constraints really represents a bound on the average decay rate $\bar{\lambda}$ since the formation of the meteorites. This implies in particular that the redshift at which one should consider this constraint depends on the specific functional dependence $\lambda(t)$. It was shown that well-designed time dependence for $\lambda$ can obviate this limit, due to the time average.

\subsubsection{Conclusions}

Meteorites data allow to set constraints on the variation of the fundamental constants, which are comparable to the ones set by the Oklo phenomenon. Similar constraints can also bet set from spontaneous fission (see Section III.A.3 of FVC [500]) but this process is less well understood and less sensitive than the $\alpha$ - and $\beta$ - decay processes and.

From an experimental point of view, the main difficulty concerns the dating of the meteorites and the interpretation of the effective decay rate.

As long as we only consider $\alpha_{\mathrm{EM}}$, the sensitivities can be computed mainly by considering the contribution of the Coulomb energy to the decay energy, that reduces to its contribution to the nuclear energy. However, as for the Oklo phenomenon, the dependencies in the other constants, $X_{\mathrm{q}}, G_{\mathrm{F}}, \mu \ldots$, require a nuclear model and remain very model-dependent.

\subsection{Quasar absorption spectra}

\subsubsection{Generalities}

Quasar (QSO) absorption lines provide a powerful probe of the variation of fundamental constants. Absorption lines in intervening clouds along the line of sight of the QSO give access to the spectra of the atoms present in the cloud, that it is to paleo-spectra. The method was first used by Savedoff [447] who constrained the time variation of the fine-structure constraint from the doublet separations seen in galaxy emission spectra. For general introduction to these observations, we refer to $[412,474,271]$.

Indeed, one cannot use a single transition compared to its laboratory value since the expansion of the universe induces a global redshifting of all spectra. In order to tackle down a variation of the fundamental constants, one should resort on various transitions and look for chromatic effects that can indeed not be reproduce by the expansion of the universe, which acts chromatically on all wavelengths.

To achieve such a test, one needs to understand the dependencies of different types of transitions, in a similar way as for atomic clock experiments. [175, 169] suggested to use the convenient formulation

$$
\omega=\omega_{0}+q\left[\left(\frac{\alpha_{\mathrm{EM}}}{\alpha_{\mathrm{EM}}^{(0)}}\right)^{2}-1\right]+q_{2}\left[\left(\frac{\alpha_{\mathrm{EM}}}{\alpha_{\mathrm{EM}}^{(0)}}\right)^{4}-1\right],
$$

in order to take into account the dependence of the spectra on the fine-structure constant. $\omega$ is the energy in the rest-frame of the cloud, that is at a redshift $z, \omega_{0}$ is the energy measured today in the 
laboratory. $q$ and $q_{2}$ are two coefficients that determine the frequency dependence on a variation of $\alpha_{\mathrm{EM}}$ and that arise from the relativistic corrections for the transition under consideration. The coefficient $q$ is typically an order of magnitude larger than $q_{2}$ so that the possibility to constrain a variation of the fine-structure constant is mainly determined by $q$. These coefficients were computed for a large set of transitions, first using a relativistic Hartree-Fock method and then using manybody perturbation theory. We refer to $[175,45,14]$ for an extensive discussion of the computational methods and a list of the $q$-coefficients for various transitions relevant for both quasar spectra and atomic clock experiments. Figure 3 summarizes some of these results. The uncertainty in $q$ are typically smaller than $30 \mathrm{~cm}^{-1}$ for $\mathrm{Mg}, \mathrm{Si}, \mathrm{Al}$ and $\mathrm{Zn}$, but much larger for $\mathrm{Cr}, \mathrm{Fe}$ and $\mathrm{Ni}$ due to their more complicated electronic configurations. The accuracy for $\omega_{0}$ from dedicated laboratory measurements now reach $0.004 \mathrm{~cm}^{-1}$. It is important to stress that the form (70) ensures that errors in the $q$-coefficients cannot lead to a non zero detection of $\Delta \alpha_{\mathrm{EM}}$.

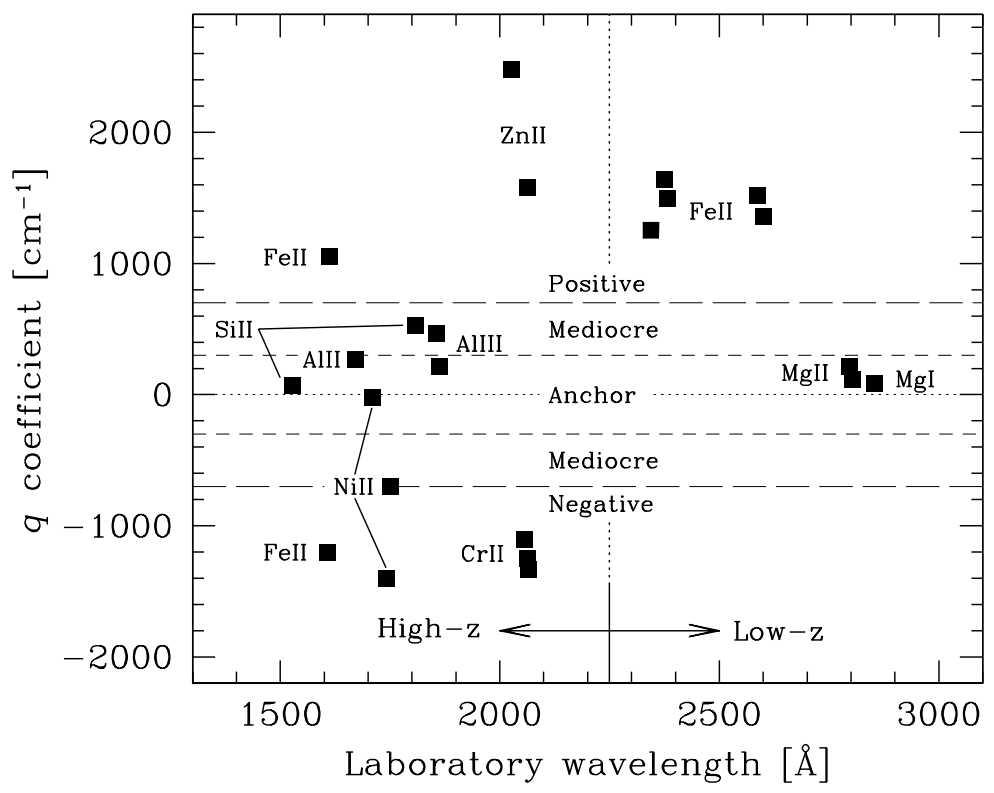

Figure 3: Summary of the values of some coefficients entering the parameterization (70) and necessary to interpret the QSO absorption spectra data. From [367]

The shift between two lines is easier to measure when the difference between the $q$-coefficients of the two lines is large, which occurs, e.g., for two levels with large $q$ of opposite sign. Many methods were developed to take this into account. The alkali doublet method (AD) focuses on the fine-structure doublet of alkali atoms. It was then generalized to the many-multiplet method (MM), which uses correlations between various transitions in different atoms. As can be seen on Figure 3, some transitions are almost insensitive to a variation of $\alpha_{\mathrm{EM}}$. This is the case of Mg II, which can be used as an anchor, i.e., a reference point. To obtain strong constraints one can either compare transitions of light atoms with those of heavy atoms (because the $\alpha_{\mathrm{EM}}$ dependence of the ground state scales as $Z^{2}$ ) or compare $s-p$ and $d-p$ transitions in heavy elements (in that case, the relativistic correction will be of opposite signs). This latter effect increases the sensitivity and strengthens the method against systematic errors. However, the results of this method rely on two assumptions: (i) ionization and chemical homogeneity and (ii) isotopic abundance of $\mathrm{Mg}$ II close to the terrestrial value. Even though these are reasonable assumptions, one cannot completely rule out systematic biases that they could induce. The AD method completely avoids the assumption of homogeneity because, by construction, the two lines of the doublet must have the same profile. 
Indeed the $\mathrm{AD}$ method avoids the implicit assumption of the MM method that chemical and ionization inhomogeneities are negligible. Another way to avoid the influence of small spectral shift due to ionization inhomogeneities within the absorber and due to possible non-zero offset between different exposures was to rely on different transitions of a single ion in individual exposure. This method has been called the Single ion differential alpha measurement method (SIDAM).

Most studies are based on optical techniques due to the profusion of strong UV transitions that are redshifted into the optical band (this includes AD, MM, SIDAM and it implies that they can be applied only above a given redshift, e.g., Si IV at $z>1.3$, Fe II $\lambda 1608$ at $z>1$ ) or on radio techniques since radio transitions arise from many different physical effects (hyperfine splitting and in particular H I $21 \mathrm{~cm}$ hyperfine transition, molecular rotation, Lambda-doubling, etc). In the latter case, the line frequencies and their comparisons yield constraints on different sets of fundamental constants including $\alpha_{\mathrm{EM}}, g_{\mathrm{p}}$ and $\mu$. Thus, these techniques are complementary since systematic effects are different in optical and radio regimes. Also the radio techniques offer some advantages: (1) to reach high spectral resolution $(<1 \mathrm{~km} / \mathrm{s})$, alleviating in particular problems with line blending and the use of, e.g., masers allow to reach a frequency calibration better than roughly $10 \mathrm{~m} / \mathrm{s}$; (2) in general, the sensitivity of the line position to a variation of a constant is higher; (3) the isotopic lines are observed separately, while in optical there is a blend with possible differential saturations (see, e.g., [109] for a discussion).

Let us first emphasize that the shifts in the absorption lines to be detected are extremely small. For instance a change of $\alpha_{\mathrm{EM}}$ of order $10^{-5}$ corresponds a shift of at most $20 \mathrm{~m} \AA$ for a redshift of $z \sim 2$, which would corresponds to a shift of order $\sim 0.5 \mathrm{~km} / \mathrm{s}$, or to about a third of a pixel at a spectral resolution of $R \sim 40000$, as achieved with Keck/HIRES or VLT/UVES. As we shall discuss later, there are several sources of uncertainty that hamper the measurement. In particular, the absorption lines have complex profiles (because they result from the propagation of photons through a highly inhomogeneous medium) that are fitted using a combination of Voigt profiles. Each of these components depends on several parameters including the redshift, the column density and the width of the line (Doppler parameter) to which one now needs to add the constants that are assumed to be varying. These parameters are constrained assuming that the profiles are the same for all transitions, which is indeed a non-trivial assumption for transitions from different species (this was one of the driving motivations to use the transition from a single species and of the SIDAM method). More important, the fit is usually not unique. This is not a problem when the lines are not saturated but it can increase the error on $\alpha_{\mathrm{EM}}$ by a factor 2 in the case of strongly saturated lines [91].

\subsubsection{Alkali doublet method (AD)}

The first method used to set constraint on the time variation of the fine-structure constant relies on fine-structure doublets splitting for which

$$
\Delta \nu \propto \frac{\alpha_{\mathrm{EM}}^{2} Z^{4} R_{\infty}}{2 n^{3}} .
$$

It follows that the relative separation is proportional $\alpha_{\mathrm{EM}}, \Delta \nu / \bar{\nu} \propto \alpha_{\mathrm{EM}}^{2}$ so that the variation of the fine structure constant at a redshift $z$ can be obtained as

$$
\left(\frac{\Delta \alpha_{\mathrm{EM}}}{\alpha_{\mathrm{EM}}}\right)(z)=\frac{c_{r}}{2}\left[\left(\frac{\Delta \lambda}{\bar{\lambda}}\right)_{z} /\left(\frac{\Delta \lambda}{\bar{\lambda}}\right)_{0}-1\right]
$$

where $c_{r} \sim 1$ is a number taking into account the relativistic corrections. This expression is indeed a simple approach of the alkali doublet since one should, as for atomic clocks, take into account the relativistic corrections more precisely. Using the formulation (70), one can deduce that

$$
c_{r}=\frac{\delta q+\delta q_{2}}{\delta q+2 \delta q_{2}}
$$

Living Reviews in Relativity

http: //www. livingreviews.org/lrr-2011-2 
where the $\delta q$ are the differences between the $q$-coefficients for the doublet transitions.

Several authors have applied the AD method to doublets of several species such as, e.g., C IV, Nv, O vi, Mg II, Al III, Si II, Si IV. We refer to Section III.3 of FVC [500] for a summary of their results (see also [318]) and focus on the three most recent analysis, based on the Si IV doublet. In this particular case, $q=766$ (resp. 362) $\mathrm{cm}^{-1}$ and $q_{2}=48$ (resp. $\left.{ }^{-8}\right) \mathrm{cm}^{-1}$ for Si IV $\lambda 1393$ (resp. $\lambda 1402$ ) so that $c_{r}=0.8914$. The method is based on a $\chi^{2}$ minimization of multiple component Voigt profile fits to the absorption features in the QSO spectra. In general such a profile depends on three parameters, the column density $N$, the Doppler width $(b)$ and the redshift. It is now extended to include $\Delta \alpha_{\mathrm{EM}} / \alpha_{\mathrm{EM}}$. The fit is carried out by simultaneously varying these parameters for each component.

- Murphy et al. [377] analyzed 21 Keck/HIRES Si IV absorption systems toward 8 quasars to obtain the weighted mean of the sample,

$$
\Delta \alpha_{\mathrm{EM}} / \alpha_{\mathrm{EM}}=(-0.5 \pm 1.3) \times 10^{-5}, \quad 2.33<z<3.08,
$$

with a mean redshift of $z=2.6$. The $\mathrm{S} / \mathrm{N}$ ratio of these data is in the range $15-40$ per pixel and the spectral resolution is $R \sim 34000$.

- Chand et al. [91] analyzed 15 Si IV absorption systems selected from a ESO-UVES sample containing 31 systems (eliminating contaminated, saturated or very broad systems; in particular a lower limit on the column density was fixed so that both lines of the doublets are detected at more than $5 \sigma$ ) to get the weighted mean,

$$
\Delta \alpha_{\mathrm{EM}} / \alpha_{\mathrm{EM}}=(-0.15 \pm 0.43) \times 10^{-5}, \quad 1.59<z<2.92 .
$$

The improvement of the constraint arises mainly from a better $\mathrm{S} / \mathrm{N}$ ratio, of order $60-80$ per pixel, and resolution $R \sim 45000$. Note that combining this result with the previous one (71 in a weighted mean would lead to $\Delta \alpha_{\mathrm{EM}} / \alpha_{\mathrm{EM}}=(-0.04 \pm 0.56) \times 10^{-5}$ in the range $1.59<z<3.02$

- The analysis [349] of seven CIV systems and two Si IV systems in the direction of a single quasar, obtained by the VLT-VES (during the science verification) has led to

$$
\Delta \alpha_{\mathrm{EM}} / \alpha_{\mathrm{EM}}=(-3.09 \pm 8.46) \times 10^{-5}, \quad 1.19<z<1.84 .
$$

This is less constraining than the two previous analyses, mainly because the $q$-coefficients are smaller for C IV (see [410] for the calibration of the laboratory spectra)

One limitation may arise from the isotopic composition of silicium. Silicium has three naturally occurring isotopes with terrestrial abundances ${ }^{28} \mathrm{Si}^{29}{ }^{29}{ }^{30} \mathrm{Si}=92.23: 4.68: 3.09$ so that each absorption line is a composite of absorption lines from the three isotopes. However, it was shown that this effect of isotopic shifts [377] is negligible in the case of Si IV.

\subsubsection{Many multiplet method (MM)}

A generalization of the $\mathrm{AD}$ method, known as the many-mulptiplet was proposed in [176]. It relies on the combination of transitions from different species. In particular, as can be seen on Figure 3, some transitions are fairly unsensitive to a change of the fine-structure constant (e.g., Mg II or MgI, hence providing good anchors) while others such as Fe II are more sensitive. The first implementation [522] of the method was based on a measurement of the shift of the Fe II (the rest wavelengths of which are very sensitive to $\alpha_{\mathrm{EM}}$ ) spectrum with respect to the one of $\mathrm{Mg}$ II. This comparison increases the sensitivity compared with methods using only alkali doublets. Two series of analyses were performed during the past ten years and lead to contradictory conclusions. The accuracy of the measurements depends on how well the absorption line profiles are modeled. 
Keck/HIRES data. The MM-method was first applied in [522] who analyzed one transition of the Mg II doublet and five Fe II transitions from three multiplets. Using 30 absorption systems toward 17 quasars, they obtained

$$
\begin{aligned}
\Delta \alpha_{\mathrm{EM}} / \alpha_{\mathrm{EM}} & =(-0.17 \pm 0.39) \times 10^{-5}, & & 0.6<z<1 \\
\Delta \alpha_{\mathrm{EM}} / \alpha_{\mathrm{EM}} & =(-1.88 \pm 0.53) \times 10^{-5}, & & 1<z<1.6 .
\end{aligned}
$$

This was the first claim that a constant may have varied during the evolution of the universe. It was later confirmed in a re-analysis $[376,524]$ of the initial sample and by including new optical QSO data to reach 28 absorption systems with redshift $z=0.5-1.8$ plus 18 damped Lyman- $\alpha$ absorption systems towards 13 QSO plus 21 Si IV absorption systems toward 13 QSO. The analysis used mainly the multiplets of Ni II, Cr II and Zn II and Mg I, Mg I, Al II, Al III and Fe II was also included. The most recent analysis [369] relies on 128 absorption spectra, later updated [367] to include 143 absorption systems. The more robust estimates is the weighted mean

$$
\Delta \alpha_{\mathrm{EM}} / \alpha_{\mathrm{EM}}=(-0.57 \pm 0.11) \times 10^{-5}, \quad 0.2<z<4.2 .
$$

The resolution for most spectra was $R \sim 45000$ and the $\mathrm{S} / \mathrm{N}$ per pixel ranges from 4 to 240 , with most spectral regions with $\mathrm{S} / \mathrm{N} \sim 30$. The wavelength scale was calibrated by mean of a thoriumargon emission lamp. This calibration is crucial and its quality is discussed in [368, 374] for the Keck/HIRES (see also [236]) as well as [534] for the VLT/UVES measurements.

The low- $z(z<1.8)$ and high- $z$ rely on different ions and transitions with very different $\alpha_{\mathrm{EM}^{-}}$ dependencies. At low- $z$, the $\mathrm{Mg}$ transitions are used as anchors against which the large positive shifts in the Fe II can be measured. At high- $z$, different transitions are fitted (Fe II, S II, Cr II, Ni II, $\mathrm{ZnII}, \mathrm{Al}$ II, AlIII). The two sub-samples respond differently to simple systematic errors due to their different arrangement of $q$-coefficients in wavelength space. The analysis for each sample give the weighted mean

$$
\begin{array}{cc}
\Delta \alpha_{\mathrm{EM}} / \alpha_{\mathrm{EM}}=(-0.54 \pm 0.12) \times 10^{-5}, & 0.2<z<1.8 \\
\Delta \alpha_{\mathrm{EM}} / \alpha_{\mathrm{EM}}=(-0.74 \pm 0.17) \times 10^{-5}, & 1.8<z<4.2
\end{array}
$$

with respectively 77 and 66 systems.

Hunting systematics. While performing this kind of observations a number of problems and systematic effects have to be taken into account and controlled. (1) Errors in the determination of laboratory wavelengths to which the observations are compared. (2) While comparing wavelengths from different atoms one has to take into account that they may be located in different regions of the cloud with different velocities and hence with different Doppler shifts. (3) One has to ensure that there is no transition not blended by transitions of another system. (4) The differential isotopic saturation has to be controlled. Usually quasar absorption systems are expected to have lower heavy element abundances. The spatial inhomogeneity of these abundances may also play a role. (5) Hyperfine splitting can induce a saturation similar to isotopic abundances. (6) The variation of the velocity of the Earth during the integration of a quasar spectrum can also induce differential Doppler shift. (7) Atmospheric dispersion across the spectral direction of the spectrograph slit can stretch the spectrum. It was shown that, on average, this can, for low redshift observations, mimic a negative $\Delta \alpha_{\mathrm{EM}} / \alpha_{\mathrm{EM}}$, while this is no more the case for high redshift observations (hence emphasizing the complementarity of these observations). (8) The presence of a magnetic field will shift the energy levels by Zeeman effect. (9) Temperature variations during the observation will change the air refractive index in the spectrograph. In particular, flexures in the instrument are dealt with by recording a calibration lamp spectrum before and after the science exposure and the signal-to-noise and stability of the lamp is crucial (10) Instrumental effects such as variations of the intrinsic instrument profile have to be controlled.

Living Reviews in Relativity

http: //www . livingreviews . org/lrr-2011-2 
All these effects have been discussed in detail in [374, 376] to argue that none of them can explain the current detection. This was recently complemented by a study on the calibration since adistortion of the wavelength scale could lead to a non-zero value of $\Delta \alpha_{\mathrm{EM}}$. The quality of the calibration is discussed in [368] and shown to have a negligible effect on the measurements (a similar result has been obtained for the VLT/UVES data [534]).

As we pointed out earlier, one assumption of the method concerns the isotopic abundances of Mg II that can affect the low- $z$ sample since any changes in the isotopic composition will alter the value of effective rest-wavelengths. This isotopic composition is assumed to be close to terrestrial ${ }^{24} \mathrm{Mg}:{ }^{25} \mathrm{Mg}:{ }^{26} \mathrm{Mg}=79: 10: 11$. No direct measurement of $r_{\mathrm{Mg}}=\left({ }^{26} \mathrm{Mg}+{ }^{25} \mathrm{Mg}\right) /{ }^{24} \mathrm{Mg}$ in QSO absorber is currently feasible due to the small separation of the isotopic absorption lines. However, it was shown [231], on the basis of molecular absorption lines of $\mathrm{MgH}$ that $r_{\mathrm{Mg}}$ generally decreases with decreasing metallicity. In standard models it should be near 0 at zero metallicity since type II supernovae are primarily producers of ${ }^{24} \mathrm{Mg}$. It was also argued that ${ }^{13} \mathrm{C}$ is a tracer of ${ }^{25} \mathrm{Mg}$ and was shown to be low in the case of HE 0515-4414 [321]. However, contrary to this trend, it was found [552] that $r_{\mathrm{Mg}}$ can reach high values for some giant stars in the globular cluster NGC 6752 with metallicity $[\mathrm{Fe} / \mathrm{H}] \sim-1.6$. This led Ashenfelter et al. [18] to propose a chemical evolution model with strongly enhanced population of intermediate $\left(2-8 M_{\odot}\right)$ stars, which in their asymptotic giant branch phase are the dominant factories for heavy $\mathrm{Mg}$ at low metallicities typical of QSO absorption systems, as a possible explanation of the low- $z$ Keck/HIRES observations without any variation of $\alpha_{\mathrm{EM}}$. It would require that $r_{\mathrm{Mg}}$ reaches 0.62 , compared to 0.27 (but then the UVES/VLT constraints would be converted to a detection). Care needs to be taken since the star formation history can be different ine each region, even in each absorber, so that one cannot a priori use the best-fit obtained from the Keck data to the UVES/VLT data. However, such modified nucleosynthetic history will lead to an overproduction of elements such as $\mathrm{P}, \mathrm{Si}, \mathrm{Al}, \mathrm{P}$ above current constraints [192], but this later model is not the same as the one of Ref. [18] that was tuned to avoid these problems.

In conclusion, no compelling evidence for a systematic effect has been raised at the moment.

VLT/UVES data. The previous results, and their importance for fundamental physics, led another team to check this detection using observations from UVES spectrograph operating on the VLT. In order to avoid as much systematics as possible, and based on numerical simulations, they apply a series of selection criteria [90] on the systems used to constrain the time variation of the fine-structure constant: (1) consider only lines with similar ionization potentials (Mg II, Fe II, Si II and $\mathrm{Al}$ II) as they are most likely to originate from similar regions in the cloud; (2) avoid absorption lines contaminated by atmospheric lines; (3) consider only systems with hight enough column density to ensure that all the mutiplets are detected at more than $5 \sigma ;(4)$ demand than at least one of the anchor lines is not saturated to have a robust measurement of the redshift; (5) reject strongly saturated systems with large velocity spread; (6) keep only systems for which the majority of the components are separated from the neighboring by more than the Doppler shift parameter.

The advantage of this choice is to reject most complex or degenerate systems, which could result in uncontrolled systematics effects. The drawback is indeed that the analysis will be based on less systems.

Refs. [90, 470] analyzed the observations of 23 absorption systems, fulfilling the above criteria, in direction of 18 QSO with a S/N ranging between 50 and 80 per pixel and a resolution $R>44000$. They concluded that

$$
\Delta \alpha_{\mathrm{EM}} / \alpha_{\mathrm{EM}}=(-0.06 \pm 0.06) \times 10^{-5}, \quad 0.4<z<2.3,
$$

hence giving a $3 \sigma$ constraint on a variation of $\alpha_{\mathrm{EM}}$. 
This analysis was challenged by Murphy, Webb and Flambaum [372, 371, 370]. Using (quoting them) the same reduced data, using the same fits to the absorption profiles, they claim to find different individual measurements of $\Delta \alpha_{\mathrm{EM}} / \alpha_{\mathrm{EM}}$ and a weighted mean,

$$
\Delta \alpha_{\mathrm{EM}} / \alpha_{\mathrm{EM}}=(-0.44 \pm 0.16) \times 10^{-5}, \quad 0.4<z<2.3,
$$

which differs from the above cited value. The main points that were raised are (1) the fact that some of the uncertainties on $\Delta \alpha_{\mathrm{EM}} / \alpha_{\mathrm{EM}}$ are smaller than a minimum uncertainty that they estimated and (2) the quality of the statistical analysis (in particular on the basis of the $\chi^{2}$ curves). These arguments were responded in [471] The revision [471] of the VLT/UVES constraint rejects two more than $4 \sigma$ deviant systems that were claimed to dominate the re-analysis [371, 370] and concludes that

$$
\Delta \alpha_{\mathrm{EM}} / \alpha_{\mathrm{EM}}=(0.01 \pm 0.15) \times 10^{-5}, \quad 0.4<z<2.3,
$$

emphasizing that the errors are probably larger.

On the basis of the articles [372, 371, 370] and the answer [471], it is indeed difficult (without having played with the data) to engage one of the parties. This exchange has enlightened some differences in the statistical analysis.

To finish, let us mention that [361] reanalyzed some systems of [90, 470] by means of the SIDAM method (see below) and disagree with some of them, claiming for a problem of calibration. They also claim that the errors quoted in [367] are underestimated by a factor 1.5.

Regressional MM (RMM). The MM method was adapted to use a linear regression method [427]. The idea is to measure the redshift $z_{i}$ deduced from the transition $i$ and plot $z_{i}$ as a function of the sensitivity coefficient. If $\Delta \alpha_{\mathrm{EM}} \neq 0$ then there should exist a linear relation with a slope proportional to $\Delta \alpha_{\mathrm{EM}} / \alpha_{\mathrm{EM}}$. On a single absorption system (VLT/UVES), on the basis of Fe II transition, they concluded that

$$
\Delta \alpha_{\mathrm{EM}} / \alpha_{\mathrm{EM}}=\left(-0.4 \pm 1.9 \pm 2.7_{\mathrm{syst}}\right) \times 10^{-6}, \quad z=1.15,
$$

compared to $\Delta \alpha_{\mathrm{EM}} / \alpha_{\mathrm{EM}}=(0.1 \pm 1.7) \times 10^{-6}$ that is obtained with the standard MM technique on the same data. This is also consistent with the constraint (79) obtained on the same system with the HARPS spectrograph.

Open controversy. At the moment, we have to face a situation in which two teams have performed two independent analyses based on data sets obtained by two instruments on two telescopes. Their conclusions do not agree, since only one of them is claiming for a detection of a variation of the fine-structure constant. This discrepancy between VLT/UVES and Keck/Hires results is yet to be resolved. In particular, they use data from a different telescopes observing a different (Southern/Northern) hemisphere.

Ref. [236] provides an analysis of the wavelength accuracy of the Keck/HIRES spectrograph. An absolute uncertainty of $\Delta z \sim 10^{-5}$, corresponding to $\Delta \lambda \sim 0.02 \AA$ with daily drift of $\Delta z \sim 5 \times 10^{-6}$ and multiday drift of $\Delta z \sim 2 \times 10^{-5}$. While the cause of this drift remains unknown, it is argued [236] that this level of systematic uncertainty makes it difficult to use the Keck/HIRES to constrain the time variation of $\alpha_{\mathrm{EM}}$ (at least for a single system or a small sample since the distortion pattern pertains to the echelle orders as they are recorded on the CDD, that is it is similar from exposure to exposure, the effect on $\Delta \alpha_{\mathrm{EM}} / \alpha_{\mathrm{EM}}$ for an ensemble of absorbers at different redshifts would be random since the transitions fall in different places with respect to the pattern of the disortion). This needs to be confirmed and investigated in more detail. We refer to [373] for a discussion on the Keck wavelength calibration error and [534] for the VLT/UVES as well as [86] for a discussion on the ThAr calibration. 
On the one hand, it is appropriate that one team has reanalyzed the data of the other and challenged its analysis. This would indeed lead to an improvement in the robustness of these results. Indeed a similar reverse analysis would also be appropriate. On the other hand both teams have achieved an amazing work in order to understand and quantify all sources of systematics. Both developments, as well as the new techniques, which are appearing, should hopefully set this observational issue. Today, it is unfortunately premature to choose one data set compared to the other.

A recent data [523] set of 60 quasar spectra (yielding 153 absorption systems) for the VLT was used and split at $z=1.8$ to get

$$
\left(\Delta \alpha_{\mathrm{EM}} / \alpha_{\mathrm{EM}}\right)_{\mathrm{VLT} ; z<1.8}=(-0.06 \pm 0.16) \times 10^{-5},
$$

in agreement with the former study [471], while at higher redshift

$$
\left(\Delta \alpha_{\mathrm{EM}} / \alpha_{\mathrm{EM}}\right)_{\mathrm{VLT} z>1.8}=(+0.61 \pm 0.20) \times 10^{-5}
$$

This higher component exhibits a positive variation of $\alpha_{\mathrm{EM}}$, that is of opposite sign with respect to the previous Keck/HIRES detection [367]

$$
\left(\Delta \alpha_{\mathrm{EM}} / \alpha_{\mathrm{EM}}\right)_{\mathrm{Keck} ; z<1.8}=(-0.54 \pm 0.12) \times 10^{-5}, \quad\left(\Delta \alpha_{\mathrm{EM}} / \alpha_{\mathrm{EM}}\right)_{\mathrm{Keck} ; z>1.8}=(-0.74 \pm 0.17) \times 10^{-5} .
$$

It was pointed out that the Keck/HIRES and VLT/UVES observations can be made consistent in the case the fine structure constant is spatially varying [523]. Indeed, one can note that they do not correspond to the same hemisphere and invoke a spatial variation. [523] concludes that the distribution of $\alpha_{\mathrm{EM}}$ is well represented by a spatial dipole, significant at $4.1 \sigma$, in the direction right ascension $17.3 \pm 0.6$ hours and declination $-61 \pm 9 \mathrm{deg}$ (see also [50, 48]). This emphasizes the difficulty in comparing different data sets and shows that the constraints can easily be combined as long as they are compatible with no variation but one must care about a possible spatial variation otherwise.

\subsubsection{Single ion differential measurement (SIDAM)}

This method [320] is an adaptation of the MM method in order to avoid the influence of small spectral shifts due to ionization inhomogeneities within the absorbers as well as to non-zero offsets between different exposures. It was mainly used with Fe II, which provides transitions with positive and negative $q$-coefficients (see Figure 3). Since it relies on a single ion, it is less sensitive to isotopic abundances, and in particular not sensitive to the one of $\mathrm{Mg}$.

The first analysis relies on the QSO HE 0515-4414 that was used in [427] to get the constraint (77). An independent analysis [361] of the same system gave a weighted mean

$$
\Delta \alpha_{\mathrm{EM}} / \alpha_{\mathrm{EM}}=(-0.12 \pm 1.79) \times 10^{-6}, \quad z=1.15
$$

at $1 \sigma$. The same system was studied independently, using the HARPS spectrograph mounted on the $3.6 \mathrm{~m}$ telescope at La Silla observatory [92]. The HARPS spectrograph has a higher resolution that UVES; $R \sim 112000$. Observations based on Fe II with a $\mathrm{S} / \mathrm{N}$ of about $30-40$ per pixel set the constraint

$$
\Delta \alpha_{\mathrm{EM}} / \alpha_{\mathrm{EM}}=(0.5 \pm 2.4) \times 10^{-6}, \quad z=1.15
$$

The second constraint [325, 361] is obtained from an absorption system toward Q 1101-264,

$$
\Delta \alpha_{\mathrm{EM}} / \alpha_{\mathrm{EM}}=(5.66 \pm 2.67) \times 10^{-6}, \quad z=1.84,
$$

These constraints do not seem to be compatible with the results of the Keck/HIRES based on the MM method. A potential systematic uncertainty, which can affect these constraints is the relative shift of the wavelength calibration in the blue and the red arms of UVES where the distant Fe lines are recorded simultaneously (see, e.g., [359] for a discussion of the systematics of this analysis). 


\subsubsection{H I-21 cm vs. UV: $x=\alpha_{\mathrm{EM}}^{2} g_{\mathrm{p}} / \mu$}

The comparison of UV heavy element transitions with the hyperfine H I transition allows to extract [496]

$$
x \equiv \alpha_{\mathrm{EM}}^{2} g_{\mathrm{p}} / \mu,
$$

since the hyperfine transition is proportional to $\alpha_{\mathrm{EM}}^{2} g_{\mathrm{p}} \mu^{-1} R_{\infty}$ while optical transitions are simply proportional to $R_{\infty}$. It follows that constraints on the time variation of $x$ can be obtained from high resolution $21 \mathrm{~cm}$ spectra compared to UV lines, e.g., of Si II, Fe II and/or Mg II, as first performed in [548] in $z \sim 0.524$ absorber.

Using 9 absorption systems, there was no evidence for any variation of $x$ [494],

$$
\Delta x / x=(-0.63 \pm 0.99) \times 10^{-5}, \quad 0.23<z<2.35
$$

This constraint was criticised in [275] on the basis that the systems have multiple components and that it is not necessary that the strongest absorption arises in the same component in both type of lines. However, the error analysis of [494] tries to estimate the effect of the assumption that the strongest absorption arises in the same component.

Following [147], we note that the systems lie in two widely-separated ranges and that the two samples have completely different scatter. Therefore it can be split into two samples of respectively 5 and 4 systems to get

$$
\begin{aligned}
& \Delta x / x=(1.02 \pm 1.68) \times 10^{-5}, \quad 0.23<z<0.53, \\
& \Delta x / x=(0.58 \pm 1.94) \times 10^{-5}, \quad 1.7<z<2.35 \text {. }
\end{aligned}
$$

In such an approach two main difficulties arise: (1) the radio and optical source must coincide (in the optical QSO can be considered pointlike and it must be checked that this is also the case for the radio source), (2) the clouds responsible for the $21 \mathrm{~cm}$ and UV absorptions must be localized in the same place. Therefore, the systems must be selected with care and today the number of such systems is small and are actively looked for [411].

The recent detection of $21 \mathrm{~cm}$ and molecular hydrogen absorption lines in the same damped Lyman- $\alpha$ system at $z_{\text {abs }}=3.174$ towards SDSS J1337 +3152 constrains [472] the variation $x$ to

$$
\Delta x / x=-(1.7 \pm 1.7) \times 10^{-6}, \quad z=3.174 .
$$

This system is unique since it allows for $21 \mathrm{~cm}, \mathrm{H}_{2}$ and $\mathrm{UV}$ observation so that in principle one can measure $\alpha_{\mathrm{EM}}, x$ and $\mu$ independently. However, as the $\mathrm{H}_{2}$ column density was low, only Werner band absorption lines are seen so that the range of sensitivity coefficients is too narrow to provide a stringent constraint, $\Delta \mu / \mu<4 \times 10^{-4}$. It was also shown that the $\mathrm{H}_{2}$ and $21 \mathrm{~cm}$ are shifted because of the inhomogeneity of the gas, hence emphasizing this limitation. [411] also mentioned that 4 systems at $z=1.3$ sets $\Delta x / x=(0.0 \pm 1.5) \times 10^{-6}$ and that another system at $z=3.1$ gives $\Delta x / x=(0.2 \pm 0.5) \times 10^{-6}$. Note also that the comparison [274] with $\mathrm{C}_{\mathrm{I}}$ at $z \sim 1.4-1.6$ towards Q0458-020 and Q2337-011, yields $\Delta x / x=(6.8 \pm 1.0) \times 10^{-6}$ over the band o redshift $0<\langle z\rangle \leq 1.46$, but this analysis ignores an important wavelength calibration estimated to be of the order of $6.7 \times 10^{-6}$. It was argued that, using the existing constraints on $\Delta \mu / \mu$, this measurement is inconsistent with claims of a smaller value of $\alpha_{\mathrm{EM}}$ from the many-multiplet method, unless fractional changes in $g_{p}$ are larger than those in $\alpha_{\mathrm{EM}}$ and $\mu$.

\subsubsection{H I vs. molecular transitions: $y \equiv g_{\mathrm{p}} \alpha_{\mathrm{EM}}^{2}$}

The H I $21 \mathrm{~cm}$ hyperfine transition frequency is proportional to $g_{\mathrm{p}} \mu^{-1} \alpha_{\mathrm{EM}}^{2} R_{\infty}$ (see Section 3.1.1). On the other hand, the rotational transition frequencies of diatomic are inversely proportional to 
their reduced mass $M$. As on the example of Equation (35) where we compared an electronic transition to a vibro-rotational transition, the comparison of the hyperfine and rotational frequencies is proportional to

$$
\frac{\nu_{\mathrm{hf}}}{\nu_{\mathrm{rot}}} \propto g_{\mathrm{p}} \alpha_{\mathrm{EM}}^{2} \frac{M}{m_{\mathrm{p}}} \simeq g_{\mathrm{p}} \alpha_{\mathrm{EM}}^{2} \equiv y,
$$

where the variation of $M / m_{\mathrm{p}}$ is usually suppressed by a large factor of the order of the ratio between the proton mass and nucleon binding energy in nuclei, so that we can safely neglect it.

The constraint on the variation of $y$ is directly determined by comparing the redshift as determined from $\mathrm{H}$ I and molecular absorption lines,

$$
\frac{\Delta y}{y}=\frac{z_{\mathrm{mol}}-z_{\mathrm{H}}}{1+z_{\mathrm{mol}}} .
$$

This method was first applied [513] to the CO molecular absorption lines [536] towards PKS $1413+135$ to get

$$
\Delta y / y=(-4 \pm 6) \times 10^{-5} \quad z=0.247 .
$$

The most recent constraint [375] relies on the comparison of the published redshifts of two absorption systems determined both from $\mathrm{H}$ I and molecular absorption. The first is a system at $z=0.6847$ in the direction of TXS $0218+357$ for which the spectra of $\mathrm{CO}(1-2),{ }^{13} \mathrm{CO}(1-2), \mathrm{C}^{18} \mathrm{O}(1-2)$, $\mathrm{CO}(2$ $3), \mathrm{HCO}^{+}(1-2)$ and $\mathrm{HCN}(1-2)$ are available. They concluded that

$$
\Delta y / y=(-0.16 \pm 0.54) \times 10^{-5} \quad z=0.6847 .
$$

The second system is an absorption system in direction of PKS $1413+135$ for which the molecular lines of $\mathrm{CO}(1-2), \mathrm{HCO}^{+}(1-2)$ and $\mathrm{HCO}^{+}(2-3)$ have been detected. The analysis led to

$$
\Delta y / y=(-0.2 \pm 0.44) \times 10^{-5}, \quad z=0.247 .
$$

[78] obtains the constraints $|\Delta y / y|<3.4 \times 10^{-5}$ at $z \sim 0.25$ and $z \sim 0.685$.

The radio domain has the advantage of heterodyne techniques, with a spectral resolution of $10^{6}$ or more, and dealing with cold gas and narrow lines. The main systematics is the kinematical bias, i.e., that the different lines do not come exactly from the same material along the line of sight, with the same velocity. To improve this method one needs to find more sources, which may be possible with the radio telescope ALMA ${ }^{3}$.

\section{$3.4 .7 \mathrm{OH}-18 \mathrm{~cm}: F=g_{\mathrm{p}}\left(\alpha_{\mathrm{EM}}^{2} \mu\right)^{1.57}$}

Using transitions originating from a single species, like with SIDAM, allows to reduce the systematic effects. The $18 \mathrm{~cm}$ lines of the $\mathrm{OH}$ radical offers such a possibility [95, 272].

The ground state, ${ }^{2} \Pi_{3 / 2} J=3 / 2$, of $\mathrm{OH}$ is split into two levels by $\Lambda$-doubling and each of these doubled level is further split into two hyperfine-structure states. Thus, it has two "main" lines $(\Delta F=0)$ and two "satellite" lines $(\Delta F=1)$. Since these four lines arise from two different physical processes $(\Lambda$-doubling and hyperfine splitting), they enjoy the same Rydberg dependence but different $g_{\mathrm{p}}$ and $\alpha_{\mathrm{EM}}$ dependencies. By comparing the four transitions to the H I hyperfine line, one can have access to

$$
F \equiv g_{\mathrm{p}}\left(\alpha_{\mathrm{EM}}^{2} \mu\right)^{1.57}
$$

and it was also proposed to combine them with $\mathrm{HCO}^{+}$transitions to lift the degeneracy.

\footnotetext{
3 http://www.eso.org/sci/facilities/alma/
} 
Using the four $18 \mathrm{~cm} \mathrm{OH}$ lines from the gravitational lens at $z \sim 0.765$ toward PMN J0134-0931 and comparing the $\mathrm{H}$ I $21 \mathrm{~cm}$ and $\mathrm{OH}$ absorption redshifts of the different components allowed to set the constraint [276]

$$
\Delta F / F=\left(-0.44 \pm 0.36 \pm 1.0_{\text {syst }}\right) \times 10^{-5}, \quad z=0.765
$$

where the second error is due to velocity offsets between $\mathrm{OH}$ and $\mathrm{H}$ I assuming a velocity dispersion of $3 \mathrm{~km} / \mathrm{s}$. A similar analysis [138] in a system in the direction of PKS 1413+135 gave

$$
\Delta F / F=(0.51 \pm 1.26) \times 10^{-5}, \quad z=0.2467
$$

\subsubsection{Far infrared fine-structure lines: $F^{\prime}=\alpha_{\mathrm{EM}}^{2} \mu$}

Another combination [300] of constants can be obtained from the comparison of far infrared finestructure spectra with rotational transitions, which respectively behaves as $R_{\infty} \alpha_{\mathrm{EM}}^{2}$ and $R_{\infty} \bar{\mu}=$ $R_{\infty} / \mu$ so that they give access to

$$
F^{\prime}=\alpha_{\mathrm{EM}}^{2} \mu \text {. }
$$

A good candidate for the rotational lines is $\mathrm{CO}$ since it is the second most abundant molecule in the Universe after $\mathrm{H}_{2}$.

Using the C II fine-structure and CO rotational emission lines from the quasars J1148+5251 and BR 1202-0725, it was concluded that

$$
\begin{array}{ll}
\Delta F^{\prime} / F^{\prime}=(0.1 \pm 1.0) \times 10^{-4}, & z=6.42, \\
\Delta F^{\prime} / F^{\prime}=(1.4 \pm 1.5) \times 10^{-5}, & z=4.69,
\end{array}
$$

which represents the best constraints at high redshift. As usual, when comparing the frequencies of two different species, one must account for random Doppler shifts caused by non-identical spatial distributions of the two species. Several other candidates for microwave and FIR lines with good sensitivities are discussed in [299].

\subsection{9 "Conjugate" satellite $\mathrm{OH}$ lines: $G=g_{\mathrm{p}}\left(\alpha_{\mathrm{EM}} \mu\right)^{1.85}$}

The satellite $\mathrm{OH} 18 \mathrm{~cm}$ lines are conjugate so that the two lines have the same shape, but with one line in emission and the other in absorption. This arises due to an inversion of the level of populations within the ground state of the $\mathrm{OH}$ molecule. This behavior has recently been discovered at cosmological distances and it was shown [95] that a comparison between the sum and difference of satellite line redshifts probes $G=g_{\mathrm{p}}\left(\alpha_{\mathrm{EM}} \mu\right)^{1.85}$.

From the analysis of the two conjugate satellite $\mathrm{OH}$ systems at $z \sim 0.247$ towards PKS $1413+135$ and at $z \sim 0.765$ towards PMN J0134-0931, it was concluded [95] that

$$
|\Delta G / G|<7.6 \times 10^{-5}
$$

It was also applied to a nearby system, Centaurus A, to give $|\Delta G / G|<1.6 \times 10^{-5}$ at $z \sim 0.0018$. A more recent analysis [273] claims for a tentative evidence (with $2.6 \sigma$ significance, or at $99.1 \%$ confidence) for a smaller value of $G$

$$
\Delta G / G=(-1.18 \pm 0.46) \times 10^{-5}
$$

for the system at $z \sim 0.247$ towards PKS $1413+135$.

One strength of this method is that it guarantees that the satellite lines arise from the same gas, preventing from velocity offset between the lines. Also, the shape of the two lines must agree if they arise from the same gas.

Living Reviews in Relativity

http: //www. livingreviews.org/lrr-2011-2 


\subsubsection{Molecular spectra and the electron-to-proton mass ratio}

As was pointed out in Section 3.1, molecular lines can provide a test of the variation ${ }^{4}$ [488] of $\mu$ since rotational and vibrational transitions are respectively inversely proportional to their reduce mass and its square-root [see Equation (35)].

\section{Constraints with $\mathrm{H}_{2}$}

$\mathrm{H}_{2}$ is the most abundant molecule in the universe and there were many attempts to use its absorption spectra to put constraints on the time variation of $\mu$ despite the fact that $\mathrm{H}_{2}$ is very difficult to detect [387].

As proposed in [512], the sensitivity of a vibro-rotational wavelength to a variation of $\mu$ can be parameterized as

$$
\lambda_{i}=\lambda_{i}^{0}\left(1+z_{\mathrm{abs}}\right)\left(1+K_{i} \frac{\Delta \mu}{\mu}\right),
$$

where $\lambda_{i}^{0}$ is the laboratory wavelength (in the vacuum) and $\lambda_{i}$ is the wavelength of the transition $i$ in the rest-frame of the cloud, that is at a redshift $z_{\text {abs }}$ so that the observed wavelength is $\lambda_{i} /\left(1+z_{\text {abs }}\right)$. $K_{i}$ is a sensitivity coefficient analogous to the $q$-coefficient introduced in Equation (70), but with different normalization since in the parameterization we would have $q_{i}=\omega_{i}^{0} K_{i} / 2$,

$$
K_{i} \equiv \frac{\mathrm{d} \ln \lambda_{i}}{\mathrm{~d} \ln \mu}
$$

corresponding to the Lyman and Werner bands of molecular hydrogen. From this expression, one can deduce that the observed redshift measured from the transition $i$ is simply

$$
z_{i}=z_{\mathrm{abs}}+b K_{i}, \quad b \equiv-\left(1+z_{\mathrm{abs}}\right) \frac{\Delta \mu}{\mu},
$$

which implies in particular that $z_{\mathrm{abs}}$ is not the mean of the $z_{i}$ if $\Delta \mu \neq 0$. Indeed $z_{i}$ is measured with some uncertainty of the astronomical measurements $\lambda_{i}$ and by errors of the laboratory measurements $\lambda_{i}^{0}$. But if $\Delta \mu \neq 0$ there must exist a correlation between $z_{i}$ and $K_{i}$ so that a linear regression of $z_{i}$ (measurement) as a function of $K_{i}$ (computed) allows to extract $\left(z_{\mathrm{abs}}, b\right)$ and their statistical significance.

We refer to Section V.C of FVC [500] for earlier studies and we focus on the latest results. The recent constraints are mainly based on the molecular hydrogen of two damped Lyman- $\alpha$ absorption systems at $z=2.3377$ and 3.0249 in the direction of two quasars (Q 1232+082 and Q 0347-382) for which a first analysis of VLT/UVES data showed [262] a slight indication of a variation,

$$
\Delta \mu / \mu=(5.7 \pm 3.8) \times 10^{-5}
$$

at $1.5 \sigma$ for the combined analysis. The lines were selected so that they are isolated, unsaturated and unblended. It follows that the analysis relies on 12 lines (over 50 detected) for the first quasar and 18 (over 80) for second but the two selected spectra had no transition in common. The authors performed their analysis with two laboratory catalogs and got different results. They point out that the errors on the laboratory wavelengths are comparable to those of the astronomical measurements.

It was further improved with an analysis of two absorption systems at $z=2.5947$ and $z=3.0249$ in the directions of Q 0405-443 and Q 0347-383 observed with the VLT/UVES spectrograph. The data have a resolution $R=53000$ and a $\mathrm{S} / \mathrm{N}$ ratio ranging between 30 and 70 . The same selection

\footnotetext{
${ }^{4}$ Again, $\mu$ is used either from $m_{\mathrm{e}} / m_{\mathrm{p}}$ or $m_{\mathrm{p}} / m_{\mathrm{e}}$. I have chosen to use $\mu=m_{\mathrm{p}} / m_{\mathrm{e}}$ and $\bar{\mu}=m_{\mathrm{e}} / m_{\mathrm{p}}$.
} 
criteria where applied, letting respectively 39 (out of 40) and 37 (out of 42) lines for each spectrum and only 7 transitions in common. The combined analysis of the two systems led [261]

$$
\Delta \mu / \mu=(1.65 \pm 0.74) \times 10^{-5} \quad \text { or } \quad \Delta \mu / \mu=(3.05 \pm 0.75) \times 10^{-5},
$$

according to the laboratory measurements that were used. The same data were reanalyzed with new and highly accurate measurements of the Lyman bands of $\mathrm{H}_{2}$, which implied a reevaluation of the sensitivity coefficient $K_{i}$. It leads to the two constraints [431]

$$
\begin{array}{ll}
\Delta \mu / \mu=(2.78 \pm 0.88) \times 10^{-5}, & z=2.59, \\
\Delta \mu / \mu=(2.06 \pm 0.79) \times 10^{-5}, & z=3.02,
\end{array}
$$

leading to a $3.5 \sigma$ detection for the weighted mean $\Delta \mu / \mu=(2.4 \pm 0.66) \times 10^{-5}$. The authors of [431] do not claim for a detection and are cautious enough to state that systematics dominate the measurements. The data of the $z=3.02$ absorption system were re-analyzed in [529], which claim that they lead to the bound $|\Delta \mu / \mu|<4.9 \times 10^{-5}$ at a $2 \sigma$ level, instead of Equation (95). Adding a new set of 6 spectra, it was concluded that $\Delta \mu / \mu=(15 \pm 14) \times 10^{-6}$ for the weighted fit $[530]$.

These two systems were reanalyzed [289], adding a new system in direction of Q 0528-250,

$$
\begin{aligned}
\Delta \mu / \mu & =(1.01 \pm 0.62) \times 10^{-5}, & & z=2.59, \\
\Delta \mu / \mu & =(0.82 \pm 0.74) \times 10^{-5}, & & z=2.8, \\
\Delta \mu / \mu & =(0.26 \pm 0.30) \times 10^{-5}, & & z=3.02,
\end{aligned}
$$

respectively with 52, 68 and 64 lines. This gives a weighted mean of $(2.6 \pm 3.0) \times 10^{-6}$ at $z \sim 2.81$. To compare with the previous data, the analysis of the two quasars in common was performed by using the same lines (this implies adding 3 and removing 16 for Q 0405-443 and adding 4 and removing 35 for Q 0347-383) to get respectively $(-1.02 \pm 0.89) \times 10^{-5}(z=2.59)$ and $(-1.2 \pm 1.4) \times 10^{-5}$ $(z=3.02)$. Both analyses disagree and this latter analysis indicates a systematic shift of $\Delta \mu / \mu$ toward 0. A second re-analysis of the same data was performed in [490, 489] using a different analysis method to get

$$
\Delta \mu / \mu=(-7 \pm 8) \times 10^{-6}
$$

Recently discovered molecular transitions at $z=2.059$ toward the quasar J2123-0050 observed by the Keck telescope allow to obtain $86 \mathrm{H}_{2}$ transitions and $7 \mathrm{HD}$ transitions to conclude [342]

$$
\Delta \mu / \mu=\left(5.6 \pm 5.5_{\text {stat }} \pm 2.7_{\text {syst }}\right) \times 10^{-6}, \quad z=2.059 .
$$

This method is subject to important systematic errors among which (1) the sensitivity to the laboratory wavelengths (since the use of two different catalogs yield different results [431]), (2) the molecular lines are located in the Lyman- $\alpha$ forest where they can be strongly blended with intervening H I Lyman- $\alpha$ absorption lines, which requires a careful fitting of the lines [289] since it is hard to find lines that are not contaminated. From an observational point of view, very few damped Lyman- $\alpha$ systems have a measurable amount of $\mathrm{H}_{2}$ so that only a dozen systems is actually known even though more systems will be obtained soon [411]. To finish, the sensitivity coefficients are usually low, typically of the order of $10^{-2}$. Some advantages of using $\mathrm{H}_{2}$ arise from the fact there are several hundred available $\mathrm{H}_{2}$ lines so that many lines from the same ground state can be used to eliminate different kinematics between regions of different excitation temperatures. The overlap between Lyman and Werner bands also allow to reduce the errors of calibration.

To conclude, the combination of all the existing observations indicate that $\mu$ is constant at the $10^{-5}$ level during the past 11 Gigayrs while an improvement of a factor 10 can be expected in the five coming years.

Living Reviews in Relativity

http: //www . livingreviews . org/lrr-2011-2 


\section{Other constraints}

It was recently proposed [201, 202] that the inversion spectrum of ammonia allows for a better sensitivity to $\mu$. The inversion vibro-rotational mode is described by a double well with the first two levels below the barrier. The tunneling implies that these two levels are split in inversion doublets. It was concluded that the inversion transitions scale as $\nu_{\text {inv }} \sim \bar{\mu}^{4.46}$, compared with a rotational transition, which scales as $\nu_{\text {rot }} \sim \bar{\mu}$. This implies that the redshifts determined by the two types of transitions are modified according to $\delta z_{\mathrm{inv}}=4.46\left(1+z_{\mathrm{abs}}\right) \Delta \mu / \mu$ and $\delta z_{\mathrm{rot}} \sim\left(1+z_{\mathrm{abs}}\right) \Delta \mu / \mu$ so that

$$
\Delta \mu / \mu=0.289 \frac{z_{\mathrm{inv}}-z_{\mathrm{rot}}}{1+z_{\mathrm{abs}}} .
$$

Only one quasar absorption system, at $z=0.68466$ in the direction of $\mathrm{B} 0218+357$, displaying $\mathrm{NH}_{3}$ is currently known and allows for this test. A first analysis [201] estimated from the published redshift uncertainties that a precision of $\sim 2 \times 10^{-6}$ on $\Delta \mu / \mu$ can be achieved. A detailed measurement [366] of the ammonia inversion transitions by comparison to $\mathrm{HCN}$ and $\mathrm{HCO}^{+}$rotational transitions concluded that

$$
|\Delta \mu / \mu|<1.8 \times 10^{-6}, \quad z=0.685,
$$

at a $2 \sigma$ level. Recently the analysis of the comparison of $\mathrm{NH}_{3}$ to $\mathrm{HC}_{3} \mathrm{~N}$ spectra was performed toward the gravitational lens system PKS 1830-211 $(z \simeq 0.89)$, which is a much more suitable system, with 10 detected $\mathrm{NH}_{3}$ inversion lines and a forest of rotational transitions. It reached the conclusion that

$$
|\Delta \mu / \mu|<1.4 \times 10^{-6}, \quad z=0.89,
$$

at a $3 \sigma$ level [250]. From a comparison of the ammonia inversion lines with the $\mathrm{NH}_{3}$ rotational transitions, it was concluded [353]

$$
|\Delta \mu / \mu|<3.8 \times 10^{-6}, \quad z=0.89,
$$

at $95 \%$ C.L. One strength of this analysis is to focus on lines arising from only one molecular species but it was mentioned that the frequencies of the inversion lines are about 25 times lower than the rotational ones, which might cause differences in the absorbed background radio continuum.

This method was also applied [323] in the Milky Way, in order to constrain the spatial variation of $\mu$ in the galaxy (see Section 6.1.3). Using ammonia emission lines from interstellar molecular clouds (Perseus molecular core, the Pipe nebula and the infrared dark clouds) it was concluded that $\Delta \mu=(4-14) \times 10^{-8}$. This indicates a positive velocity offset between the ammonia inversion transition and rotational transitions of other molecules. Two systems being located toward the galactic center while one is in the direction of the anti-center, this may indicate a spatial variation of $\mu$ on galactic scales.

\section{New possibilities}

The detection of several deuterated molecular hydrogen HD transitions makes it possible to test the variation of $\mu$ in the same way as with $\mathrm{H}_{2}$ but in a completely independent way, even though today it has been detected only in 2 places in the universe. The sensitivity coefficients have been published in [263] and HD was first detected by [387].

$\mathrm{HD}$ was recently detected [473] together with $\mathrm{CO}$ and $\mathrm{H}_{2}$ in a DLA cloud at a redshift of 2.418 toward SDSS1439+11 with 5 lines of HD in 3 components together with several $\mathrm{H}_{2}$ lines in 7 components. It allowed to set the $3 \sigma$ limit of $|\Delta \mu / \mu|<9 \times 10^{-5}$ [412].

Even though the small number of lines does not allow to reach the level of accuracy of $\mathrm{H}_{2}$ it is a very promising system in particular to obtain independent measurements. 


\subsubsection{Emission spectra}

Similar analysis to constrain the time variation of the fundamental constants were also performed with emission spectra. Very few such estimates have been performed, since it is less sensitive and harder to extend to sources with high redshift. In particular, emission lines are usually broad as compared to absorption lines and the larger individual errors need to be beaten by large statistics.

The O III doublet analysis [24] from a sample of 165 quasars from SDSS gave the constraint

$$
\Delta \alpha_{\mathrm{EM}} / \alpha_{\mathrm{EM}}=(12 \pm 7) \times 10^{-5}, \quad 0.16<z<0.8
$$

The method was then extended straightforwardly along the lines of the MM method and applied [238] to the fine-structure transitions in Ne III, Ne V, O III, O I and S II multiplets from a sample of 14 Seyfert 1.5 galaxies to derive the constraint

$$
\Delta \alpha_{\mathrm{EM}} / \alpha_{\mathrm{EM}}=(150 \pm 70) \times 10^{-5}, \quad 0.035<z<0.281 .
$$

\subsubsection{Conclusion and prospects}

This subsection illustrates the diversity of methods and the progresses that have been achieved to set robust constraints on the variation of fundamental constants. Many systems are now used, giving access to different combinations of the constants. It exploits a large part of the electromagnetic spectrum from far infrared to ultra violet and radio bands and optical and radio techniques have played complementary roles. The most recent and accurate constraints are summarized in Table 10 and Figure 4.
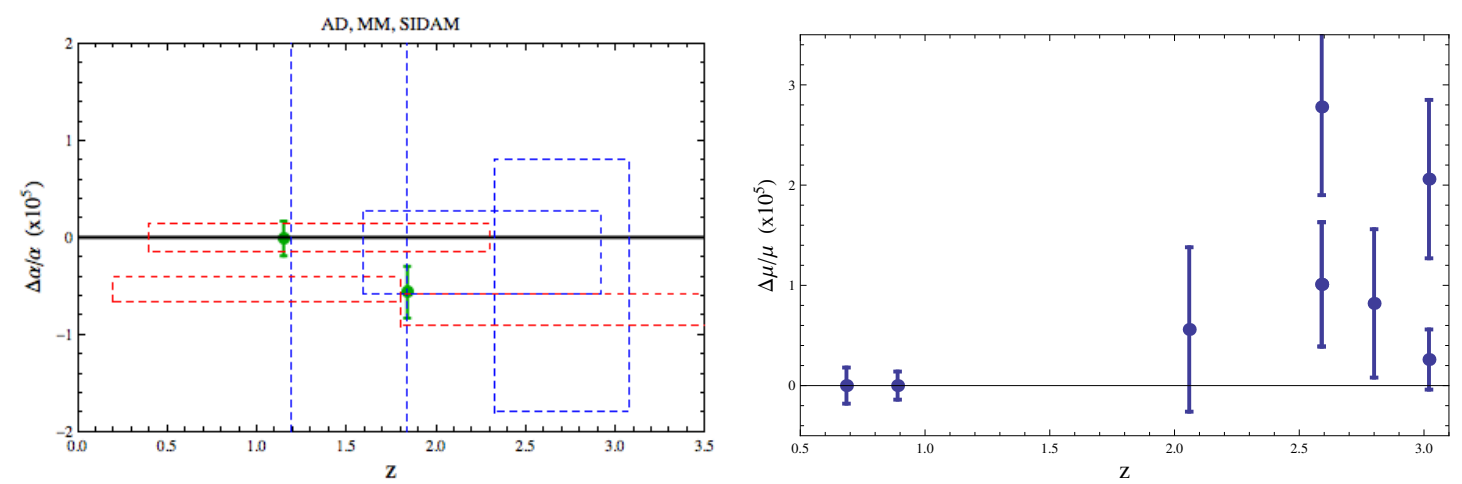

Figure 4: Summary of the direct constraints on $\alpha_{\mathrm{EM}}$ obtained from the AD (blue), MM (red) and AD (green) methods (left) and on $\mu$ (right) that are summarized in Table 10.

At the moment, only one analysis claims to have detected a variation of the fine structure constant (Keck/HIRES) while the VLT/UVES points toward no variation of the fine structure constant. It has led to the proposition that $\alpha_{\mathrm{EM}}$ may be space dependent and exhibit a dipole, the origin of which is not explained. Needless to say that such a controversy and hypotheses are sane since it will help improve the analysis of this data, but it is premature to conclude on the issue of this debate and the jury is still out. Most of the systematics have been investigated in detail and now seem under control.

Let us what we can learn on the physics from these measurement. As an example, consider the constraints obtained on $\mu, y$ and $F$ in the redshift band $0.6-0.8$ (see Table 10). They can be used to extract independent constraints on $g_{\mathrm{p}}, \alpha_{\mathrm{EM}}$ and $\mu$

$\Delta \mu / \mu=(0 \pm 0.18) \times 10^{-5}, \quad \Delta \alpha_{\mathrm{EM}} / \alpha_{\mathrm{EM}}=(-0.27 \pm 2.09) \times 10^{-5}, \quad \Delta g_{\mathrm{p}} / g_{\mathrm{p}}=(0.38 \pm 4.73) \times 10^{-5}$ 
Table 10: Summary of the latest constraints on the variation of fundamental constants obtained from the analysis of quasar absorption spectra. We recall that $y \equiv g_{\mathrm{p}} \alpha_{\mathrm{EM}}^{2}, F \equiv g_{\mathrm{p}}\left(\alpha_{\mathrm{EM}}^{2} \mu\right)^{1.57}, x \equiv \alpha_{\mathrm{EM}}^{2} g_{\mathrm{p}} / \mu$, $F^{\prime} \equiv \alpha_{\mathrm{EM}}^{2} \mu$ and $\mu \equiv m_{\mathrm{p}} / m_{\mathrm{e}}, G=g_{\mathrm{p}}(\alpha \mu)^{1.85}$.

\begin{tabular}{|c|c|c|c|c|c|}
\hline Constant & Method & System & Constraint $\left(\times 10^{-5}\right)$ & Redshift & Ref. \\
\hline \multirow[t]{7}{*}{$\alpha_{\mathrm{EM}}$} & $\mathrm{AD}$ & 21 & $(-0.5 \pm 1.3)$ & $2.33-3.08$ & {$[377]$} \\
\hline & $\mathrm{AD}$ & 15 & $(-0.15 \pm 0.43)$ & $1.59-2.92$ & {$[91]$} \\
\hline & $\mathrm{AD}$ & 9 & $(-3.09 \pm 8.46)$ & $1.19-1.84$ & [349] \\
\hline & MM & 143 & $(-0.57 \pm 0.11)$ & $0.2-4.2$ & {$[367]$} \\
\hline & MM & 21 & $(0.01 \pm 0.15)$ & $0.4-2.3$ & {$[90]$} \\
\hline & SIDAM & 1 & $(-0.012 \pm 0.179)$ & 1.15 & {$[361]$} \\
\hline & SIDAM & 1 & $(0.566 \pm 0.267)$ & 1.84 & {$[361]$} \\
\hline \multirow[t]{3}{*}{$y$} & $\mathrm{HI}-\mathrm{mol}$ & 1 & $(-0.16 \pm 0.54)$ & 0.6847 & {$[375]$} \\
\hline & H I - mol & 1 & $(-0.2 \pm 0.44)$ & 0.247 & {$[375]$} \\
\hline & $\mathrm{CO}, \mathrm{CHO}^{+}$ & & $(-4 \pm 6)$ & 0.247 & [536] \\
\hline \multirow[t]{2}{*}{$F$} & $\mathrm{OH}-\mathrm{HI}$ & 1 & $\left(-0.44 \pm 0.36 \pm 1.0_{\text {syst }}\right)$ & 0.765 & {$[276]$} \\
\hline & $\mathrm{OH}-\mathrm{HI}$ & 1 & $(0.51 \pm 1.26)$ & 0.2467 & [138] \\
\hline \multirow[t]{2}{*}{$x$} & H I - UV & 9 & $(-0.63 \pm 0.99)$ & $0.23-2.35$ & {$[494]$} \\
\hline & H I - UV & 2 & $(-0.17 \pm 0.17)$ & 3.174 & {$[472]$} \\
\hline \multirow[t]{2}{*}{$F^{\prime}$} & $\mathrm{C}$ II - CO & 1 & $(1 \pm 10)$ & 4.69 & {$[327]$} \\
\hline & $\mathrm{C}$ II - CO & 1 & $(14 \pm 15)$ & 6.42 & [327] \\
\hline \multirow[t]{3}{*}{$G$} & $\mathrm{OH}$ & 1 & $<1.1$ & $0.247,0.765$ & {$[95]$} \\
\hline & $\mathrm{OH}$ & 1 & $<1.16$ & 0.0018 & {$[95]$} \\
\hline & $\mathrm{OH}$ & 1 & $(-1.18 \pm 0.46)$ & 0.247 & [273] \\
\hline \multirow[t]{11}{*}{$\mu$} & $\mathrm{H}_{2}$ & 1 & $(2.78 \pm 0.88)$ & 2.59 & [431] \\
\hline & $\mathrm{H}_{2}$ & 1 & $(2.06 \pm 0.79)$ & 3.02 & [431] \\
\hline & $\mathrm{H}_{2}$ & 1 & $(1.01 \pm 0.62)$ & 2.59 & [289] \\
\hline & $\mathrm{H}_{2}$ & 1 & $(0.82 \pm 0.74)$ & 2.8 & [289] \\
\hline & $\mathrm{H}_{2}$ & 1 & $(0.26 \pm 0.30)$ & 3.02 & [289] \\
\hline & $\mathrm{H}_{2}$ & 1 & $(0.7 \pm 0.8)$ & $3.02,2.59$ & [490] \\
\hline & $\mathrm{NH}_{3}$ & 1 & $<0.18$ & 0.685 & {$[366]$} \\
\hline & $\mathrm{NH}_{3}$ & 1 & $<0.38$ & 0.685 & [353] \\
\hline & $\mathrm{HC}_{3} \mathrm{~N}$ & 1 & $<0.14$ & 0.89 & {$[250]$} \\
\hline & HD & 1 & $<9$ & 2.418 & [412] \\
\hline & HD & 1 & $\left(0.56 \pm 0.55_{\text {stat }} \pm 0.27_{\text {syst }}\right)$ & 2.059 & {$[342]$} \\
\hline
\end{tabular}


This shows that one can test the compatibility of the constraints obtained from different kind of systems. Independently of these constraints, we have seen in Section 6.3 that in grand unification theory the variation of the constants are correlated. The former constraints show that if $\Delta \ln \mu=$ $R \Delta \ln \alpha_{\mathrm{EM}}$ then the constraint (101) imposes that $\left|R \Delta \ln \alpha_{\mathrm{EM}}\right|<1.8 \times 10^{-6}$. In general $R$ is expected to be of the order of $30-50$. Even if its value its time-dependent, that would mean that $\Delta \ln \alpha_{\mathrm{EM}} \sim(1-5) \times 10^{-7}$, which is highly incompatible with the constraint $(74)$ obtained by the same team on $\alpha_{\mathrm{EM}}$, but also on the constraints (71) and (72) obtained from the AD method and on which both teams agree. This illustrates how important the whole set of data is since one will probably be able to constrain the order of magnitude of $R$ in a near future, which would be a very important piece of information for the theoretical investigations.

We mention in the course of this paragraph many possibilities to improve these constraints.

Since the AD method is free of the two main assumptions of the MM method, it seems important to increase the precision of this method as well as any method relying only on one species. This can be achieved by increasing the $\mathrm{S} / \mathrm{N}$ ratio and spectral resolution of the data used or by increasing the sample size and including new transitions (e.g., cobalt $[172,187]$ ).

The search for a better resolution is being investigated in many direction. With the current resolution of $R \sim 40000$, the observed line positions can be determined with an accuracy of $\sigma_{\lambda} \sim 1 \mathrm{~m} \AA$. This implies that the accuracy on $\Delta \alpha_{\mathrm{EM}} / \alpha_{\mathrm{EM}}$ is of the order of $10^{-5}$ for lines with typical $q$-coefficients. As we have seen this limit can be improved to $10^{-6}$ when more transitions or systems are used together. Any improvement is related to the possibility to measure line positions more accurately. This can be done by increasing $R$ up to the point at which the narrowest lines in the absorption systems are resolved. The Bohlin formula [62] gives the estimates

$$
\sigma_{\lambda} \sim \Delta \lambda_{\mathrm{pix}}\left(\frac{\Delta \lambda_{\mathrm{pix}}}{W_{\mathrm{obs}}}\right) \frac{1}{\sqrt{N_{e}}}\left(\frac{M^{3 / 2}}{\sqrt{12}}\right)
$$

where $\Delta \lambda_{\text {pix }}$ is the pixel size, $W_{\text {obs }}$ is the observed equivalent width, $N_{e}$ is the mean number of photoelectron at the continuum level and $M$ is the number of pixel covering the line profile. The metal lines have intrinsic width of a few $\mathrm{km} / \mathrm{s}$. Thus, one can expect improvements from higher spectral resolution. Progresses concerning the calibration are also expected, using, e.g., laser comb [478]. Let us just mention, the EXPRESSO (Echelle Spectrograph for PREcision Super Stable Observation) project [115] on 4 VLT units or the CODEX (COsmic Dynamics EXplorer) on E-ELT projects [360, 357, 507]. They shall provide a resolving power of $R=150000$ to be compared to the HARPS ${ }^{5}$ (High Accuracy Radial velocity planet Searcher) spectrograph $(R \sim 112000)$ has been used but it is operating on a $3.6 \mathrm{~m}$ telescope.

The limitation may then lie in the statistics and the calibration and it would be useful to use more than two QSO with overlapping spectra to cross-calibrate the line positions. This means that one needs to discover more absorption systems suited for these analyses. Much progress is expected. For instance, the FIR lines are expected to be observed by a new generation of telescopes such as HERSCHEL ${ }^{6}$. While the size of the radio sample is still small, surveys are being carried out so that the number of known redshift $\mathrm{OH}, \mathrm{HI}$ and $\mathrm{HCO}^{+}$absorption systems will increase. For instance the future Square Kilometer Array (SKA) will be able to detect relative changes of the order of $10^{-7}$ in $\alpha_{\mathrm{EM}}$.

In conclusion, it is clear that these constraints and the understanding of the absorption systems will increase in the coming years.

\footnotetext{
5 http://obswww.unige.ch/Instruments/HARPS/

6 http://sci.esa.int/science-e/www/area/index. cfm?fareaid=16
} 


\subsection{Stellar constraints}

Stars start to accumulate helium produced by the pp-reaction and the CNO cycle in their core. Furthermore, the products of further nuclear reactions of helium with either helium or hydrogen lead to isotopes with $A=5$ or $A=8$, which are highly unstable. In order to produce elements heavier than $A>7$ by fusion of lighter isotopes, the stars need to reach high temperatures and densities. In these conditions, newly produced ${ }^{12} \mathrm{C}$ would almost immediately be fused further to form heavier elements so that one expects only a tiny amount of ${ }^{12} \mathrm{C}$ to be produced, in contradiction with the observed abundances. This led Hoyle [257] to conclude that a then unknown excited state of the ${ }^{12} \mathrm{C}$ with an energy close to the $3 \alpha$-threshold should exist since such a resonance would increase the probability that ${ }^{8} \mathrm{Be}$ captures an $\alpha$-particle. It follows that the production of ${ }^{12} \mathrm{C}$ in stars relies on the three conditions:

- the decay lifetime of ${ }^{8} \mathrm{Be}$, of order $10^{-16} \mathrm{~s}$, is four orders of magnitude longer than the time for two $\alpha$ particles to scatter, so that a macroscopic amount of beryllium can be produced, which is sufficient to lead to considerable production of carbon,

- an excited state of ${ }^{12} \mathrm{C}$ lies just above the energy of ${ }^{8} \mathrm{Be}+\alpha$, which allows for

$$
{ }^{4} \mathrm{He}+{ }^{4} \mathrm{He} \leftrightarrow{ }^{8} \mathrm{Be}, \quad{ }^{8} \mathrm{Be}+{ }^{4} \mathrm{He} \leftrightarrow{ }^{12} \mathrm{C}^{*} \rightarrow{ }^{12} \mathrm{C}+7.367 \mathrm{MeV},
$$

- the energy level of ${ }^{16} \mathrm{O}$ at $7.1197 \mathrm{MeV}$ is non resonant and below the energy of ${ }^{12} \mathrm{C}+\alpha$, of order $7.1616 \mathrm{MeV}$, which ensures that most of the carbon synthesized is not destroyed by the capture of an $\alpha$-particle. The existence of this resonance, the $J_{l}^{\pi}=0_{2}^{+}$-state of ${ }^{12} \mathrm{C}$ was actually discovered [111] experimentally later, with an energy of $372 \pm 4 \mathrm{keV}$ [today, $E_{0_{2}^{+}}=379.47 \pm 0.15 \mathrm{keV}$, above the ground state of three $\alpha$-particles (see Figure 5 ).
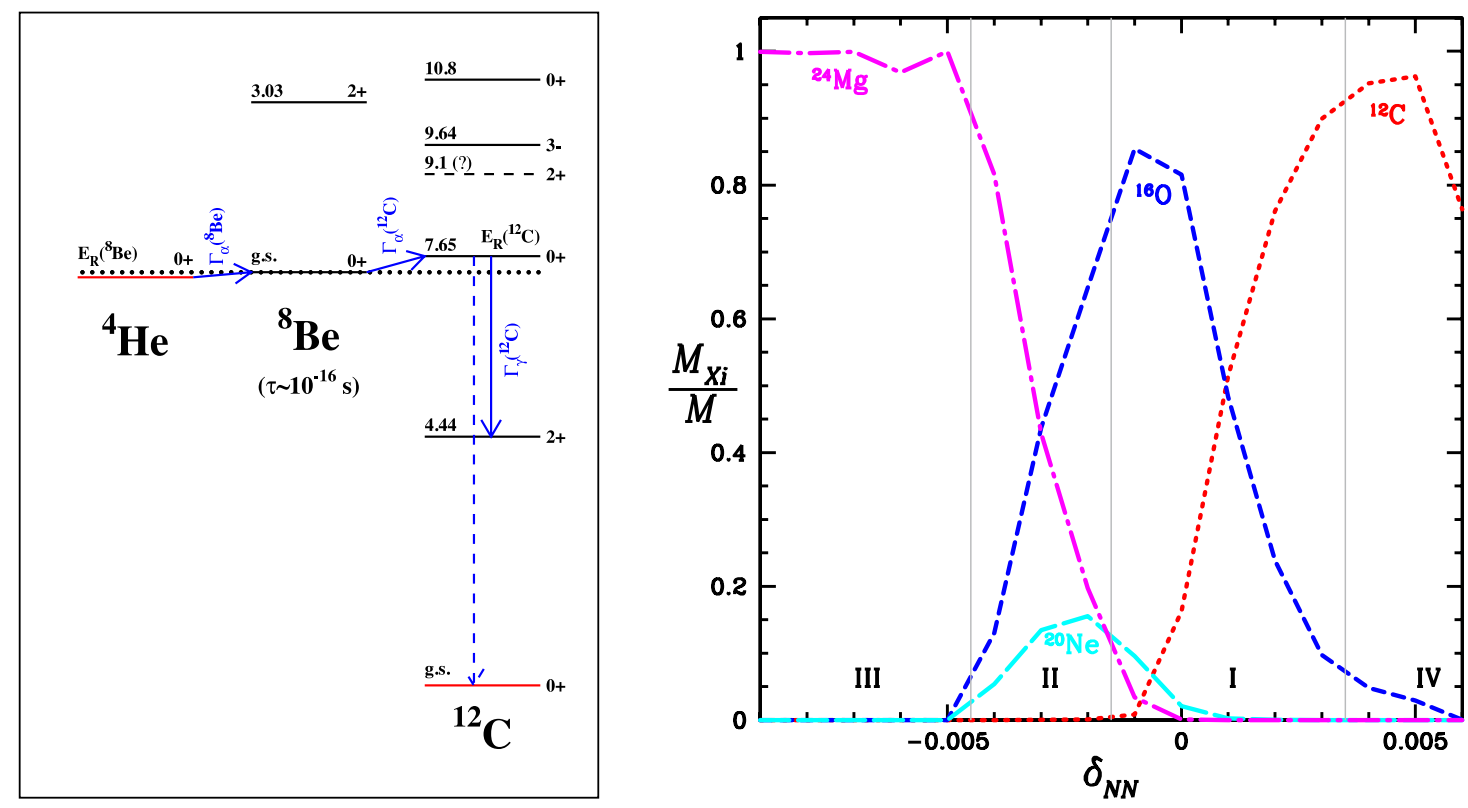

Figure 5: Left: Level scheme of nuclei participating to the ${ }^{4} \mathrm{He}(\alpha \alpha, \gamma){ }^{12} \mathrm{C}$ reaction. Right: Central abundances at the end of the CHe burning as a function of $\delta_{N N}$ for a $60 M_{\odot}$ star with $Z=0$. From [103].

The variation of any constant that would modify the energy of this resonance would also endanger the stellar nucleosynthesis of carbon, so that the possibility for carbon production has 
often been used in anthropic arguments. Qualitatively, if $E_{0_{2}^{+}}$is increased then the carbon would be rapidly processed to oxygen since the star would need to be hotter for the triple- $\alpha$ process to start. On the other hand, if $E_{0_{2}^{+}}$is decreased, then all $\alpha$-particles would produce carbon so that no oxygen would be synthesized. It was estimated [334] that the carbon production in intermediate and massive stars is suppressed if the various of the energy of the resonance is outside the range $-250 \mathrm{keV} \lesssim \Delta E_{0_{2}^{+}} \lesssim 60 \mathrm{keV}$, which was further improved [451] to, $-5 \mathrm{keV} \lesssim \Delta E_{0_{2}^{+}} \lesssim 50 \mathrm{keV}$ in order for the $\mathrm{C} / \mathrm{O}$ ratio to be larger than the error in the standard yields by more than $50 \%$. Indeed, in such an analysis, the energy of the resonance was changed by hand. However, we expect that if $E_{0_{2}^{+}}$is modified due to the variation of a constant other quantities, such as the resonance of the oxygen, the binding energies and the cross sections will also be modified in a complex way.

In practice, to draw a constraint on the variation of the fundamental constants from the stellar production of carbon, one needs to go through different steps, any of them involving assumptions,

1. to determine the effective parameters, e.g., cross sections, which affects the stellar evolution. The simplest choice is to modify only the energy of the resonance but it may not be realistic since all cross sections and binding energies should also be affected. This requires one to use a stellar evolutionary model;

2. relate these parameters to nuclear parameters. This involves the whole nuclear physics machinery;

3. to relate the nuclear parameters to fundamental constants. As for the Oklo phenomenon, it requires to link QCD to nuclear physics.

A first analysis [390, 391, 451] used a model that treats the carbon nucleus by solving the 12-nucleon Schrödinger equation using a three-cluster wavefunction representing the three-body dynamics of the ${ }^{12} \mathrm{C}$ state. The NN interaction was described by the Minnesota model [297, 491] and its strength was modified by multiplying the effective NN-potential by an arbitrary number $p$. This allows to relate the energy of the Hoyle level relative to the triple alpha threshold, $\varepsilon \equiv Q_{\alpha \alpha \alpha}$, and the gamma width, $\Gamma_{\gamma}$, as a function of the parameter $p$, the latter being almost not affected. The modified $3 \alpha$-reaction rate was then given by

$$
r_{\alpha}=3^{3 / 2} N_{\alpha}^{3}\left(\frac{2 \pi \hbar^{2}}{M_{\alpha} k_{\mathrm{B}} T}\right)^{3} \frac{\Gamma}{\hbar} \exp \left[-\frac{\varepsilon(p)}{k_{\mathrm{B}} T}\right],
$$

where $M_{\alpha}$ and $N_{\alpha}$ are the mass and number density of the $\alpha$-particle, The resonance width $\Gamma=\Gamma_{\alpha} \Gamma_{\gamma} /\left(\Gamma_{\alpha}+\Gamma_{\gamma}\right) \sim \Gamma_{\gamma}$. This was included in a stellar code and ran for red giant stars with 1.3, 5 and $20 M_{\odot}$ with solar metallicity up to thermally pulsating asymptotic giant branch [390] and in low, intermediate and high mass $\left(1.3,5,15,25 M_{\odot}\right)$ with solar metallicity also up to TP-AGB [451] to conclude that outside a window of respectively $0.5 \%$ and $4 \%$ of the values of the strong and electromagnetic forces, the stellar production of carbon or oxygen will be reduced by a factor 30 to 1000 .

In order to compute the resonance energy of the ${ }^{8} \mathrm{Be}$ and ${ }^{12} \mathrm{C}$ a microscopic cluster model was developed [297]. The Hamiltonian of the system is then of the form $H=\sum_{i}^{A} T\left(\mathbf{r}_{i}+\sum_{j<i}^{A} V\left(\mathbf{r}_{i j}\right)\right.$, where $A$ is the nucleon number, $T$ the kinetic energy and $V$ the NN interaction potential. In order to implement the variation of the strength of the nuclear interaction with respect to the electromagnetic interaction, it was taken as

$$
V\left(\mathbf{r}_{i j}\right)=V_{C}\left(\mathbf{r}_{i j}\right)+\left(1+\delta_{N N}\right) V_{N}\left(\mathbf{r}_{i j}\right),
$$

where $\delta_{N N}$ is a dimensionless parameter that describes the change of the nuclear interaction, $V_{N}$ being described in [491]. When $A>4$ no exact solution can be found and approximate solutions 
in which the wave function of the ${ }^{8} \mathrm{Be}$ and ${ }^{12} \mathrm{C}$ are described by clusters of respectively 2 and 3 $\alpha$-particle is well adapted.

First, $\delta_{N N}$ can be related to the deuterium binding energy as

$$
\Delta B_{D} / B_{D}=5.7701 \times \delta_{N N},
$$

which, given the discussion in Section 3.8.3, allows to relate $\delta_{N N}$ to fundamental constants, as, e.g., in [104]. Then, the resonance energy of the ${ }^{8} \mathrm{Be}$ and ${ }^{12} \mathrm{C}$ scale as

$$
E_{R}\left({ }^{8} \mathrm{Be}\right)=\left(0.09208-12.208 \times \delta_{N N}\right) \mathrm{Mev}, \quad E_{R}\left({ }^{12} \mathrm{C}\right)=\left(0.2877-20.412 \times \delta_{N N}\right) \mathrm{Mev},
$$

so that the energy of the Hoyle level relative to the triple alpha threshold is $Q_{\alpha \alpha \alpha}=E_{R}\left({ }^{8} \mathrm{Be}\right)+$ $E_{R}\left({ }^{12} \mathrm{C}\right)$.

This was implemented in $[103,180]$ to population III stars with typical masses, 15 and $60 M_{\odot}$ with zero metallicity, in order to compute the central abundances at the end of the core He burning. From Figure 5, one can distinguish 4 regimes (I) the star ends the CHe burning phase with a core composed of a mixture of ${ }^{12} \mathrm{C}$ and ${ }^{16} \mathrm{O}$, as in the standard case; (II) if the $3 \alpha$ rate is weaker, ${ }^{12} \mathrm{C}$ is produced slower, the reaction ${ }^{12} \mathrm{C}(\alpha, \gamma){ }^{16} \mathrm{O}$ becomes efficient earlier so that the star ends the CHe burning phase with a core composed mostly of ${ }^{16} \mathrm{O}$; (III) for weaker rates, the ${ }^{16} \mathrm{O}$ is further processed to ${ }^{20} \mathrm{Ne}$ and then ${ }^{24} \mathrm{Mg}$ so that the star ends the CHe burning phase with a core composed of ${ }^{24} \mathrm{Mg}$ and (IV) if the $3 \alpha$ rate is stronger, the ${ }^{12} \mathrm{C}$ is produced more rapidly and the star ends the CHe burning phase with a core composed mostly of ${ }^{12} \mathrm{C}$. Typically this imposes that

$$
-5 \times 10^{-4}<\delta_{N N}<1.5 \times 10^{-3}, \quad-3 \times 10^{-4}<\Delta B_{D} / B_{D}<9 \times 10^{-3},
$$

at a redshift of order $z \sim 15$, to ensure the ratio $\mathrm{C} / \mathrm{O}$ to be of order unity.

To finish, a recent study [3] focus on the existence of stars themselves, by revisiting the stellar equilibrium when the values of some constants are modified. In some sense, it can be seen as a generalization of the work by Gamow [224] to constrain the Dirac model of a varying gravitational constant by estimating its effect on the lifetime of the Sun. In this semi-analytical stellar structure model, the effect of the fundamental constants was reduced phenomenologically to 3 parameters, $G$, which enters mainly on the hydrostatic equilibrium, $\alpha_{\mathrm{EM}}$, which enters in the Coulomb barrier penetration through the Gamow energy, and a composite parameter $\mathcal{C}$, which describes globally the modification of the nuclear reaction rates. The underlying idea is to assume that the power generated per unit volume, $\varepsilon(r)$, and which determines the luminosity of the star, is proportional to the fudge factor $\mathcal{C}$, which would arise from a modification of the nuclear fusion factor, or equivalently of the cross section. Thus, it assumes that all cross sections are affected is a similar way. The parameter space for which stars can form and for which stable nuclear configurations exist was determined, showing that no fine-tuning seems to be required.

This new system is very promising and will provide new information on the fundamental constants at redshifts smaller than $z \sim 15$ where no constraints exist at the moment, even though drawing a robust constraint seems to be difficult at the moment. In particular, an underlying limitation arises from the fact that the composition of the interstellar media is a mixture of ejecta from stars with different masses and it is not clear which type of stars contribute the most the carbon and oxygen production. Besides, one would need to include rotation and mass loss [181]. As for the Oklo phenomenon, another limitation arises from the complexity of nuclear physics.

\subsection{Cosmic Microwave Background}

The CMB radiation is composed of photons emitted at the time of the recombination of hydrogen and helium when the universe was about 300,000 years old [see, e.g., [409] for details on the physics of the CMB]. This radiation is observed to be a black-body with a temperature $T_{0}=2.725 \mathrm{~K}$ with 
small anisotropies of order of the $\mu \mathrm{K}$. The temperature fluctuation in a direction $(\vartheta, \varphi)$ is usually decomposed on a basis of spherical harmonics as

$$
\frac{\delta T}{T}(\vartheta, \varphi)=\sum_{\ell} \sum_{m=-\ell}^{m=+\ell} a_{\ell m} Y_{\ell m}(\vartheta, \varphi)
$$

The angular power spectrum multipole $C_{\ell}=\left\langle\left|a_{l m}\right|^{2}\right\rangle$ is the coefficient of the decomposition of the angular correlation function on Legendre polynomials. Given a model of structure formation and a set of cosmological parameters, this angular power spectrum can be computed and compared to observational data in order to constrain this set of parameters.

The CMB temperature anisotropies mainly depend on three constants: $G, \alpha_{\mathrm{EM}}$ and $m_{\mathrm{e}}$.

The gravitational constant enters in the Friedmann equation and in the evolution of the cosmological perturbations. It has mainly three effects [435] that are detailed in Section 4.4.1. $\alpha_{\mathrm{EM}}$, $m_{\mathrm{e}}$ affect the dynamics of the recombination. Their influence is complex and must be computed numerically. However, we can trace their main effects since they mainly modify the CMB spectrum through the change in the differential optical depth of photons due to the Thomson scattering

$$
\dot{\tau}=x_{\mathrm{e}} n_{\mathrm{e}} c \sigma_{\mathrm{T}}
$$

which enters in the collision term of the Boltzmann equation describing the evolution of the photon distribution function and where $x_{\mathrm{e}}$ is the ionization fraction (i.e., the number density of free electrons with respect to their total number density $n_{\mathrm{e}}$ ).

The first dependence arises from the Thomson scattering cross section given by

$$
\sigma_{\mathrm{T}}=\frac{8 \pi}{3} \frac{\hbar^{2}}{m_{\mathrm{e}}^{2} c^{2}} \alpha_{\mathrm{EM}}^{2}
$$

and the scattering by free protons can be neglected since $m_{\mathrm{e}} / m_{\mathrm{p}} \sim 5 \times 10^{-4}$.

The second, and more subtle dependence, comes from the ionization fraction. Recombination proceeds via 2-photon emission from the $2 s$ level or via the Ly- $\alpha$ photons, which are redshifted out of the resonance line [405] because recombination to the ground state can be neglected since it leads to immediate re-ionization of another hydrogen atom by the emission of a Ly- $\alpha$ photons. Following [405, 338] and taking into account, for the sake of simplicity, only the recombination of hydrogen, the equation of evolution of the ionization fraction takes the form

$$
\frac{\mathrm{d} x_{\mathrm{e}}}{\mathrm{d} t}=\mathcal{C}\left[\beta\left(1-x_{\mathrm{e}}\right) \exp \left(-\frac{B_{1}-B_{2}}{k_{\mathrm{B}} T_{M}}\right)-\mathcal{R} n_{\mathrm{p}} x_{\mathrm{e}}^{2}\right],
$$

where $T_{M}$ is the temperature. At high redshift, $T_{M}$ is identical to the one of the photons $T_{\gamma}=$ $T_{0}(1+z)$ but evolves according to

$$
\frac{\mathrm{d} T_{M}}{\mathrm{~d} t}=-\frac{8 \sigma_{\mathrm{T}} a_{R}}{3 m_{\mathrm{e}}} T_{R}^{4} \frac{x_{\mathrm{e}}}{1+x_{\mathrm{e}}}\left(T_{M}-T_{\gamma}\right)-2 H T_{M}
$$

where the radiation constant $a_{R}=4 \sigma_{\mathrm{SB}} / c$ with $\sigma_{\mathrm{SB}}=k_{\mathrm{B}}^{4} \pi^{2} /\left(60 \pi c^{2} \hbar^{3}\right)$ the Stefan-Boltzmann constant. In Equation (113), $B_{n}=-E_{I} / n^{2}$ is the energy of the $n$th hydrogen atomic level, $\beta$ is the ionization coefficient, $\mathcal{R}$ the recombination coefficient, $\mathcal{C}$ the correction constant due to the redshift of Ly- $\alpha$ photons and to 2 -photon decay and $n_{p}=n_{e}$ is the number density of protons. $\beta$ is related to $\mathcal{R}$ by the principle of detailed balance so that

$$
\beta=\mathcal{R}\left(\frac{2 \pi m_{\mathrm{e}} k_{\mathrm{B}} T_{M}}{h^{2}}\right)^{3 / 2} \exp \left(-\frac{B_{2}}{k_{\mathrm{B}} T_{M}}\right) .
$$


The recombination rate to all other excited levels is

$$
\mathcal{R}=\frac{8 \pi}{c^{2}}\left(\frac{k_{\mathrm{B}} T}{2 \pi m_{\mathrm{e}}}\right)^{3 / 2} \sum_{n, l}^{*}(2 l+1) \mathrm{e}^{B_{n} / k_{\mathrm{B}} T} \int_{B_{n} / k_{\mathrm{B}} T}^{\infty} \sigma_{n l} \frac{y^{2} \mathrm{~d} y}{\mathrm{e}^{y}-1}
$$

where $\sigma_{n l}$ is the ionization cross section for the $(n, l)$ excited level of hydrogen. The star indicates that the sum needs to be regularized and the $\alpha_{\mathrm{EM}^{-}}, m_{\mathrm{e}}$-dependence of the ionization cross section is complicated to extract. However, it can be shown to behave as $\sigma_{n l} \propto \alpha_{\mathrm{EM}}^{-1} m_{\mathrm{e}}^{-2} f\left(h \nu / B_{1}\right)$. Finally, the factor $\mathcal{C}$ is given by

$$
\mathcal{C}=\frac{1+K \Lambda_{2 s}\left(1-x_{e}\right)}{1+K\left(\beta+\Lambda_{2 s}\right)\left(1-x_{e}\right)}
$$

where $\Lambda_{2 s}$ is the rate of decay of the $2 s$ excited level to the ground state via 2 photons; it scales as $m_{\mathrm{e}} \alpha_{\mathrm{EM}}^{8}$. The constant $K$ is given in terms of the Ly- $\alpha$ photon $\lambda_{\alpha}=16 \pi \hbar /\left(3 m_{\mathrm{e}} \alpha_{\mathrm{EM}}^{2} c\right)$ by $K=n_{p} \lambda_{\alpha}^{3} /(8 \pi H)$ and scales as $m_{\mathrm{e}}^{-3} \alpha_{\mathrm{EM}}^{-6}$.

In summary, both the temperature of the decoupling and the residual ionization after recombination are modified by a variation of $\alpha_{\mathrm{EM}}$ or $m_{\mathrm{e}}$. This was first discussed in [36, 277]. The last scattering surface can roughly be determined by the maximum of the visibility function $g=\dot{\tau} \exp (-\tau)$, which measures the differential probability for a photon to be scattered at a given redshift. Increasing $\alpha_{\mathrm{EM}}$ shifts $g$ to a higher redshift at which the expansion rate is faster so that the temperature and $x_{e}$ decrease more rapidly, resulting in a narrower $g$. This induces a shift of the $C_{\ell}$ spectrum to higher multipoles and an increase of the values of the $C_{\ell}$. The first effect can be understood by the fact that pushing the last scattering surface to a higher redshift leads to a smaller sound horizon at decoupling. The second effect results from a smaller Silk damping.

Most studies have introduced those modifications in the RECFAST code [454] including similar equations for the recombination of helium. Our previous analysis shows that the dependences in the fundamental constants have various origins, since the binding energies $B_{i}$ scale has $m_{\mathrm{e}} \alpha_{\mathrm{EM}}^{2}$, $\sigma_{T}$ as $\alpha_{\mathrm{EM}}^{2} m_{\mathrm{e}}^{-2}, K$ as $m_{\mathrm{e}}^{-3} \alpha_{\mathrm{EM}}^{-6}$, the ionisation coefficients $\beta$ as $\alpha_{\mathrm{EM}}^{3}$, the transition frequencies as $m_{\mathrm{e}} \alpha_{\mathrm{EM}}^{2}$, the Einstein's coefficients as $m_{\mathrm{e}} \alpha_{\mathrm{EM}}^{5}$, the decay rates $\Lambda$ as $m_{\mathrm{e}} \alpha_{\mathrm{EM}}^{8}$ and $\mathcal{R}$ has complicated dependence, which roughly reduces to $\alpha_{\mathrm{EM}}^{-1} m_{\mathrm{e}}^{-2}$. Note that a change in the fine-structure constant and in the mass of the electron are degenerate according to $\Delta \alpha_{\mathrm{EM}} \approx 0.39 \Delta m_{\mathrm{e}}$ but this degeneracy is broken for multipoles higher than 1500 [36]. In earlier works [244, 277] it was approximated by the scaling $\mathcal{R} \propto \alpha_{\text {EM }}^{2(1+\xi)}$ with $\xi \sim 0.7$.

The first studies $\left[244,277\right.$ ] focused on the sensitivity that can be reached by $\mathrm{WMAP}^{7}$ and Planck $^{8}$. They concluded that they should provide a constraint on $\alpha_{\mathrm{EM}}$ at recombination, i.e., at a redshift of about $z \sim 1,000$, with a typical precision $\left|\Delta \alpha_{\mathrm{EM}} / \alpha_{\mathrm{EM}}\right| \sim 10^{-2}-10^{-3}$.

The first attempt [21] to actually set a constraint was performed on the first release of the data by BOOMERanG and MAXIMA. It concluded that a value of $\alpha_{\mathrm{EM}}$ smaller by a few percents in the past was favored but no definite bound was obtained, mainly due to the degeneracies with other cosmological parameters. It was later improved [22] by a joint analysis of BBN and CMB data that assumes that only $\alpha_{\mathrm{EM}}$ varies and that included 4 cosmological parameters $\left(\Omega_{\mathrm{mat}}, \Omega_{\mathrm{b}}, h, n_{s}\right)$ assuming a universe with Euclidean spatial section, leading to $-0.09<\Delta \alpha_{\mathrm{EM}}<0.02$ at $68 \%$ confidence level. A similar analysis [307], describing the dependence of a variation of the finestructure constant as an effect on recombination the redshift of which was modeled to scale as $z_{*}=1080\left[1+2 \Delta \alpha_{\mathrm{EM}} / \alpha_{\mathrm{EM}}\right]$, set the constraint $-0.14<\Delta \alpha_{\mathrm{EM}}<0.02$, at a $2 \sigma$ level, assuming a spatially flat cosmological models with adiabatic primordial fluctuations that. The effect of reionisation was discussed in [350]. These works assume that only $\alpha_{\mathrm{EM}}$ is varying but, as can been seen from Eqs. (110-116), assuming the electron mass constant.

\footnotetext{
7 http://map.gsfc.nasa.gov/

8 http://astro.estec.esa.nl/SA-general/Projects/Planck/
} 
With the WMAP first year data, the bound on the variation of $\alpha_{\mathrm{EM}}$ was sharpened [438] to $-0.05<\Delta \alpha_{\mathrm{EM}} / \alpha_{\mathrm{EM}}<0.02$, after marginalizing over the remaining cosmological parameters $\left(\Omega_{\text {mat }} h^{2}, \Omega_{\mathrm{b}} h^{2}, \Omega h^{2}, n_{s}, \alpha_{s}, \tau\right)$ assuming a universe with Euclidean spatial sections. Restricting to a model with a vanishing running of the spectral index $\left(\alpha_{s} \equiv \mathrm{d} n_{s} / \mathrm{d} \ln k=0\right)$, it gives $-0.06<$ $\Delta \alpha_{\mathrm{EM}} / \alpha_{\mathrm{EM}}<0.01$, at a $95 \%$ confidence level. In particular it shows that a lower value of $\alpha_{\mathrm{EM}}$ makes $\alpha_{s}=0$ more compatible with the data. These bounds were obtained without using other cosmological data sets. This constraint was confirmed by the analysis of [259], which got $-0.097<\Delta \alpha_{\mathrm{EM}} \alpha_{\mathrm{EM}}<0.034$, with the WMAP-1yr data alone and $-0.042<\Delta \alpha_{\mathrm{EM}} / \alpha_{\mathrm{EM}}<0.026$, at a $95 \%$ confidence level, when combined with constraints on the Hubble parameter from the HST Hubble Key project.

The analysis of the WMAP-3yr data allows to improve [476] this bound to $-0.039<$ $\Delta \alpha_{\mathrm{EM}} / \alpha_{\mathrm{EM}}<0.010$, at a $95 \%$ confidence level, assuming $\left(\Omega_{\mathrm{mat}}, \Omega_{\mathrm{b}}, h, n_{s}, z_{\mathrm{re}}, A_{s}\right)$ for the cosmological parameters $\left(\Omega_{\Lambda}\right.$ being derived from the assumption $\Omega_{K}=0$, as well as $\tau$ from the re-ionisation redshift, $\left.z_{\mathrm{re}}\right)$ and using both temperature and polarization data $(T T, T E, E E)$.

The WMAP 5-year data were analyzed, in combination with the $2 \mathrm{dF}$ galaxy redshift survey, assuming that both $\alpha_{\mathrm{EM}}$ and $m_{\mathrm{e}}$ can vary and that the universe was spatially Euclidean. Letting 6 cosmological parameters $\left[\left(\Omega_{\text {mat }} h^{2}, \Omega_{\mathrm{b}} h^{2}, \Theta, \tau, n_{s}, A_{s}\right), \Theta\right.$ being the ratio between the sound horizon and the angular distance at decoupling] and 2 constants vary they, it was concluded [452, 453] $-0.012<\Delta \alpha_{\mathrm{EM}} / \alpha_{\mathrm{EM}}<0.018$ and $-0.068<\Delta m_{\mathrm{e}} / m_{\mathrm{e}}<0.044$, the bounds fluctuating slightly depending on the choice of the recombination scenario. A similar analyis [381] not including $m_{\mathrm{e}}$ gave $-0.050<\Delta \alpha_{\mathrm{EM}} / \alpha_{\mathrm{EM}}<0.042$, which can be reduced by taking into account some further prior from the HST data. Including polarisation data data from ACBAR, QUAD and BICEP, it was also obtained [352] $-0.043<\Delta \alpha_{\mathrm{EM}} / \alpha_{\mathrm{EM}}<0.038$ at $95 \%$ C.L. and $-0.013<\Delta \alpha_{\mathrm{EM}} / \alpha_{\mathrm{EM}}<0.015$ including HST data, also at $95 \%$ C.L. Let us also emphasize the work by [351] trying to include the variation of the Newton constant by assuming that $\Delta \alpha_{\mathrm{EM}} / \alpha_{\mathrm{EM}}=Q \Delta G / G, Q$ being a constant and the investigation of [380] taking into account $\alpha_{\mathrm{EM}}, m_{\mathrm{e}}$ and $\mu, G$ being kept fixed. Considering $\left(\Omega_{\text {mat }}, \Omega_{\mathrm{b}}, h, n_{s}, \tau\right)$ for the cosmological parameters they concluded from WMAP-5 data $(T T, T E$, $E E)$ that $-8.28 \times 10^{-3}<\Delta \alpha_{\mathrm{EM}} / \alpha_{\mathrm{EM}}<1.81 \times 10^{-3}$ and $-0.52<\Delta \mu / \mu<0.17$

The analysis of [452, 453] was updated [310] to the WMAP-7yr data, including polarisation and SDSS data. It leads to $-0.025<\Delta \alpha_{\mathrm{EM}} / \alpha_{\mathrm{EM}}<-0.003$ and $0.009<\Delta m_{\mathrm{e}} / m_{\mathrm{e}}<0.079$ at a $1 \sigma$ level.

The main limitation of these analyses lies in the fact that the CMB angular power spectrum depends on the evolution of both the background spacetime and the cosmological perturbations. It follows that it depends on the whole set of cosmological parameters as well as on initial conditions, that is on the shape of the initial power spectrum, so that the results will always be conditional to the model of structure formation. The constraints on $\alpha_{\mathrm{EM}}$ or $m_{\mathrm{e}}$ can then be seen mostly as constraints on a delayed recombination. A strong constraint on the variation of $\alpha_{\mathrm{EM}}$ can be obtained from the CMB only if the cosmological parameters are independently known. [438] forecasts that CMB alone can determine $\alpha_{\mathrm{EM}}$ to a maximum accuracy of $0.1 \%$.

\section{$3.7 \quad 21 \mathrm{~cm}$}

After recombination, the CMB photons are redshifted and their temperature drops as $(1+z)$. However, the baryons are prevented from cooling adiabatically since the residual amount of free electrons, that can couple the gas to the radiation through Compton scattering, is too small. It follows that the matter decouples thermally from the radiation at a redshift of order $z \sim 200$.

The intergalactic hydrogen atoms after recombination are in their ground state, which hyperfinestructure splits into a singlet and a triple states $\left(1 s_{1 / 2}\right.$ with $F=0$ and $F=1$ respectively, see Section III.B.1 of FCV [500]). It was recently proposed [284] that the observation of the $21 \mathrm{~cm}$ emission can provide a test on the fundamental constants. We refer to [221] for a detailed review

Living Reviews in Relativity

http://www. livingreviews.org//rr-2011-2 
Table 11: Summary of the latest constraints on the variation of fundamental constants obtained from the analysis of cosmological data and more particularly of CMB data. All assume $\Omega_{K}=0$.

\begin{tabular}{llll}
\hline $\begin{array}{l}\text { Constraint } \\
\left(\alpha_{\mathrm{EM}} \times 10^{2}\right)\end{array}$ & Data & Comment & Ref. \\
\hline$[-9,2]$ & BOOMERanG-DASI-COBE + BBN & $\begin{array}{l}\text { BBN with } \alpha_{\mathrm{EM}} \text { only } \\
\left(\Omega_{\mathrm{mat}}, \Omega_{\mathrm{b}}, h, n_{s}\right)\end{array}$ & {$[22]$} \\
& & $\left(\Omega_{\mathrm{mat}}, \Omega_{\mathrm{b}}, h, n_{s}\right)$ & {$[307]$} \\
{$[-1.4,2]$} & COBE-BOOMERanG-MAXIMA & $\left(\Omega_{\mathrm{mat}} h^{2}, \Omega_{\mathrm{b}} h^{2}, \Omega_{\Lambda} h^{2}, \tau, n_{s}, \alpha_{s}\right)$ & {$[438]$} \\
{$[-5,2]$} & WMAP-1 & same $+\alpha_{s}=0$ & {$[438]$} \\
{$[-6,1]$} & WMAP-1 & $\left(\Omega_{\mathrm{mat}}, \Omega_{\mathrm{b}}, h, n_{s}, \tau, m_{\mathrm{e}}\right)$ & {$[259]$} \\
{$[-9.7,3.4]$} & WMAP-1 & same & {$[259]$} \\
{$[-4.2,2.6]$} & WMAP-1 + HST & $\left(\Omega_{\mathrm{mat}}, \Omega_{\mathrm{b}}, h, n_{s}, z_{\mathrm{re}}, A_{s}\right)$ & {$[476]$} \\
{$[-3.9,1.0]$} & WMAP-3 (TT,TE,EE) + HST & $\left(\Omega_{\mathrm{mat}} h^{2}, \Omega_{\mathrm{b}} h^{2}, \Theta, \tau, n_{s}, A_{s}, m_{\mathrm{e}}\right)$ & {$[452]$} \\
{$[-1.2,1.8]$} & WMAP-5 + ACBAR + CBI + 2df & $\left(\Omega_{\mathrm{mat}} h^{2}, \Omega_{\mathrm{b}} h^{2}, \Theta, \tau, n_{s}, A_{s}, m_{\mathrm{e}}\right)$ & {$[453]$} \\
{$[-1.9,1.7]$} & WMAP-5 + ACBAR + CBI + 2df & $\left(\Omega_{\mathrm{mat}} h^{2}, \Omega_{\mathrm{b}} h^{2}, h, \tau, n_{s}, A_{s}\right)$ & {$[381]$} \\
{$[-5.0,4.2]$} & WMAP-5 + HST & $\left(\Omega_{\mathrm{mat}} h^{2}, \Omega_{\mathrm{b}} h^{2}, h, \tau, n_{s}\right)$ & {$[352]$} \\
{$[-4.3,3.8]$} & WMAP-5 + ACBAR + QUAD + BICEP & $\left(\Omega_{\mathrm{mat}} h^{2}, \Omega_{\mathrm{b}} h^{2}, h, \tau, n_{s}\right)$ & {$[352]$} \\
{$[-1.3,1.5]$} & WMAP-5 + ACBAR + QUAD + BICEP+HST & $\left(\Omega_{\mathrm{mat}} h^{2}, \Omega_{\mathrm{b}} h^{2}, h, \tau, n_{s}, A_{s}, m_{\mathrm{e}}, \mu\right)$ & {$[380]$} \\
{$[-0.83,0.18]$} & WMAP-5 (TT,TE,EE) & $\left(\Omega_{\mathrm{mat}} h^{2}, \Omega_{\mathrm{b}} h^{2}, \Theta, \tau, n_{s}, A_{s}, m_{\mathrm{e}}\right.$ & {$[310]$} \\
{$[-2.5,-0.3]$} & WMAP-7 + H + SDSS & &
\end{tabular}

on $21 \mathrm{~cm}$.

The fraction of atoms in the excited (triplet) state versus the ground (singlet) state is conventionally related by the spin temperature $T_{\mathrm{s}}$ defined by the relation

$$
\frac{n_{t}}{n_{s}}=3 \exp \left(-\frac{T_{*}}{T_{\mathrm{s}}}\right)
$$

where $T_{*} \equiv h c /\left(\lambda_{21} k_{\mathrm{B}}\right)=68.2 \mathrm{mK}$ is the temperature corresponding to the $21 \mathrm{~cm}$ transition and the factor 3 accounts for the degeneracy of the triplet state (note that this is a very simplified description since the assumption of a unique spin temperature is probably not correct [221]. The population of the two states is determined by two processes, the radiative interaction with CMB photons with a wavelength of $\lambda_{21}=21.1 \mathrm{~cm}$ (i.e., $\nu_{21}=1420 \mathrm{MHz}$ ) and spin-changing atomic collision. Thus, the evolution of the spin temperature is dictated by [221].

$$
\frac{\mathrm{d} T_{\mathrm{s}}}{\mathrm{d} t}=4 C_{10}\left(\frac{1}{T_{\mathrm{s}}}-\frac{1}{T_{\mathrm{g}}}\right) T_{\mathrm{s}}^{2}+(1+z) H A_{10}\left(\frac{1}{T_{\mathrm{s}}}-\frac{1}{T_{\gamma}}\right) \frac{T_{\gamma}}{T_{*}}
$$

The first term corresponds to the collision de-excitation rate from triplet to singlet and the coefficient $C_{10}$ is decomposed as

$$
C_{10}=\kappa_{10}^{H H} n_{p}+\kappa_{10}^{e H} x_{\mathrm{e}} n_{p}
$$

with the respective contribution of $\mathrm{H}-\mathrm{H}$ and $e-\mathrm{H}$ collisions. The second term corresponds to spontaneous transition and $A_{10}$ is the Einstein coefficient. The equation of evolution for the gas temperature $T_{\mathrm{g}}$ is given by Equation (114) with $T_{M}=T_{\mathrm{g}}$ (we recall that we have neglected the contribution of helium) and the electronic density satisfies Equation (113).

It follows $[284,285]$ that the change in the brightness temperature of the CMB at the corresponding wavelength scales as $T_{\mathrm{b}} \propto A_{12} / \nu_{21}^{2}$, where the Einstein coefficient $A_{12}$ is defined below. Observationally, we can deduce the brightness temperature from the brightness $I_{\nu}$, that is the energy received in a given direction per unit area, solid angle and time, defined as the temperature of the black-body radiation with spectrum $I_{\nu}$. Thus, $k_{\mathrm{B}} T_{\mathrm{b}} \simeq I_{\nu} c^{2} / 2 \nu^{2}$. It has a mean value, $\bar{T}_{\mathrm{b}}\left(z_{\text {obs }}\right)$ at various redshift where $1+z_{\text {obs }}=\nu_{21}^{\text {today }} / \nu_{\text {obs }}$. Besides, as for the CMB, there will also be fluctuation in $T_{\mathrm{b}}$ due to imprints of the cosmological perturbations on $n_{p}$ and $T_{\mathrm{g}}$. It follows that we also have access to an angular power spectrum $C_{\ell}\left(z_{\text {obs }}\right)$ at various redshift (see [329] for details on this computation). 
Both quantities depend on the value of the fundamental constants. Beside the same dependencies of the CMB that arise from the Thomson scattering cross section, we have to consider those arising from the collision terms. In natural units, the Einstein coefficient scaling is given by $A_{12}=\frac{2}{3} \pi \alpha_{\mathrm{EM}} \nu_{21}^{3} m_{\mathrm{e}}^{-2} \sim 2.869 \times 10^{-15} \mathrm{~s}^{-1}$. It follows that it scales as $A_{10} \propto g_{\mathrm{p}}^{3} \mu^{3} \alpha_{\mathrm{EM}}^{13} m_{\mathrm{e}}$. The brightness temperature depends on the fundamental constant as $T_{\mathrm{b}} \propto g_{\mathrm{p}} \mu \alpha_{\mathrm{EM}}^{5} / m_{\mathrm{e}}$. Note that the signal can also be affected by a time variation of the gravitational constant through the expansion history of the universe. [284] (see also [221] for further discussions), focusing only on $\alpha_{\mathrm{EM}}$, showed that this was the dominant effect on a variation of the fundamental constant (the effect on $C_{10}$ is much complicated to determine but was argued to be much smaller). It was estimated that a single station telescope like $\mathrm{LWA}^{9}$ or $\mathrm{LOFAR}^{10}$ can lead to a constraint of the order of $\Delta \alpha_{\mathrm{EM}} / \alpha_{\mathrm{EM}} \sim 0.85 \%$, improving to $0.3 \%$ for the full LWA. The fundamental challenge for such a measurement is the subtraction of the foreground.

The $21 \mathrm{~cm}$ absorption signal in a available on a band of redshift typically ranging from $z \lesssim 1000$ to $z \sim 20$, which is between the CMB observation and the formation of the first stars, that is during the "dark age". Thus, it offers an interesting possibility to trace the constraints on the evolution of the fundamental constants between the CMB epoch and the quasar absorption spectra.

As for $\mathrm{CMB}$, the knowledge of the cosmological parameters is a limitation since a change of $1 \%$ in the baryon density or the Hubble parameter implies a $2 \%$ (3\% respectively) on the mean bolometric temperature. The effect on the angular power spectrum have been estimated but still require an in depth analysis along the lines of, e.g., [329]. It is motivating since $C_{\ell}\left(z_{\mathrm{obs}}\right)$ is expected to depend on the correlators of the fundamental constants, e.g., $\left\langle\alpha_{\mathrm{EM}}\left(\mathbf{x}, z_{\mathrm{obs}}\right) \alpha_{\mathrm{EM}}\left(\mathbf{x}^{\prime}, z_{\mathrm{obs}}\right)\right\rangle$ and thus in principle allows to study their fluctuation, even though it will also depend on the initial condition, e.g., power spectrum, of the cosmological perturbations.

In conclusion, the $21 \mathrm{~cm}$ observation opens a observational window on the fundamental at redshifts ranging typically from 30 to 100, but full in-depth analysis is still required (see [206, 286] for a critical discussion of this probe).

\subsection{Big bang nucleosynthesis}

\subsubsection{Overview}

The amount of ${ }^{4} \mathrm{He}$ produced during the big bang nucleosynthesis is mainly determined by the neutron to proton ratio at the freeze-out of the weak interactions that interconvert neutrons and protons. The result of Big Bang nucleosynthesis (BBN) thus depends on $G, \alpha_{\mathrm{W}}, \alpha_{\mathrm{EM}}$ and $\alpha_{\mathrm{S}}$ respectively through the expansion rate, the neutron to proton ratio, the neutron-proton mass difference and the nuclear reaction rates, besides the standard parameters such as, e.g., the number of neutrino families.

The standard BBN scenario [117, 409] proceeds in three main steps:

1. for $T>1 \mathrm{MeV},(t<1 \mathrm{~s})$ a first stage during which the neutrons, protons, electrons, positrons an neutrinos are kept in statistical equilibrium by the (rapid) weak interaction

$$
n \longleftrightarrow p+e^{-}+\bar{\nu}_{e}, \quad n+\nu_{e} \longleftrightarrow p+e^{-}, \quad n+e^{+} \longleftrightarrow p+\bar{\nu}_{e}
$$

As long as statistical equilibrium holds, the neutron to proton ratio is

$$
(n / p)=\mathrm{e}^{-Q_{\mathrm{np}} / k_{\mathrm{B}} T}
$$

\footnotetext{
${ }^{9}$ http://lwa.unm.edu

10 http://www. lofar.org
} 
where $Q_{\mathrm{np}} \equiv\left(m_{\mathrm{n}}-m_{\mathrm{p}}\right) c^{2}=1.29 \mathrm{MeV}$. The abundance of the other light elements is given by [409]

$$
Y_{A}=g_{A}\left(\frac{\zeta(3)}{\sqrt{\pi}}\right)^{A-1} 2^{(3 A-5) / 2} A^{5 / 2}\left[\frac{k_{\mathrm{B}} T}{m_{\mathrm{N}} c^{2}}\right]^{3(A-1) / 2} \eta^{A-1} Y_{\mathrm{p}}^{Z} Y_{\mathrm{n}}^{A-Z} \mathrm{e}^{B_{A} / k_{\mathrm{B}} T},
$$

where $g_{A}$ is the number of degrees of freedom of the nucleus ${ }_{Z}^{A} \mathrm{X}, m_{\mathrm{N}}$ is the nucleon mass, $\eta$ the baryon-photon ratio and $B_{A} \equiv\left(Z m_{\mathrm{p}}+(A-Z) m_{\mathrm{n}}-m_{A}\right) c^{2}$ the binding energy.

2. Around $T \sim 0.8 \mathrm{MeV}(t \sim 2 \mathrm{~s})$, the weak interactions freeze out at a temperature $T_{\mathrm{f}}$ determined by the competition between the weak interaction rates and the expansion rate of the universe and thus roughly determined by $\Gamma_{\mathrm{w}}\left(T_{\mathrm{f}}\right) \sim H\left(T_{\mathrm{f}}\right)$ that is

$$
G_{\mathrm{F}}^{2}\left(k_{\mathrm{B}} T_{\mathrm{f}}\right)^{5} \sim \sqrt{G N_{*}}\left(k_{\mathrm{B}} T_{\mathrm{f}}\right)^{2}
$$

where $G_{\mathrm{F}}$ is the Fermi constant and $N_{*}$ the number of relativistic degrees of freedom at $T_{\mathrm{f}}$. Below $T_{\mathrm{f}}$, the number of neutrons and protons change only from the neutron $\beta$-decay between $T_{\mathrm{f}}$ to $T_{\mathrm{N}} \sim 0.1 \mathrm{MeV}$ when $p+n$ reactions proceed faster than their inverse dissociation.

3. For $0.05 \mathrm{MeV}<T<0.6 \mathrm{MeV}(3 \mathrm{~s}<t<6 \mathrm{~min})$, the synthesis of light elements occurs only by two-body reactions. This requires the deuteron to be synthesized $(p+n \rightarrow D)$ and the photon density must be low enough for the photo-dissociation to be negligible. This happens roughly when

$$
\frac{n_{\mathrm{d}}}{n_{\gamma}} \sim \eta^{2} \exp \left(-B_{D} / T_{\mathrm{N}}\right) \sim 1
$$

with $\eta \sim 3 \times 10^{-10}$. The abundance of ${ }^{4} \mathrm{He}$ by mass, $Y_{\mathrm{p}}$, is then well estimated by

$$
Y_{\mathrm{p}} \simeq 2 \frac{(n / p)_{\mathrm{N}}}{1+(n / p)_{\mathrm{N}}}
$$

with

$$
(n / p)_{\mathrm{N}}=(n / p)_{\mathrm{f}} \exp \left(-t_{\mathrm{N}} / \tau_{\mathrm{n}}\right)
$$

with $t_{\mathrm{N}} \propto G^{-1 / 2} T_{\mathrm{N}}^{-2}$ and $\tau_{\mathrm{n}}^{-1}=1.636 G_{\mathrm{F}}^{2}\left(1+3 g_{A}^{2}\right) m_{\mathrm{e}}^{5} /\left(2 \pi^{3}\right)$, with $g_{A} \simeq 1.26$ being the axial/vector coupling of the nucleon. Assuming that $B_{D} \propto \alpha_{S}^{2}$, this gives a dependence $t_{\mathrm{N}} / \tau_{\mathrm{p}} \propto G^{-1 / 2} \alpha_{\mathrm{S}}^{2} G_{\mathrm{F}}^{2}$.

4. The abundances of the light element abundances, $Y_{i}$, are then obtained by solving a series of nuclear reactions

$$
\dot{Y}_{i}=J-\Gamma Y_{i},
$$

where $J$ and $\Gamma$ are time-dependent source and sink terms.

From an observational point of view, the light elements abundances can be computed as a function of $\eta$ and compared to their observed abundances. Figure 6 summarizes the observational constraints obtained on helium-4, helium-3, deuterium and lithium-7. On the other hand, $\eta$ can be determined independently from the analysis of the cosmic microwave background anisotropies and the WMAP data [296] have led to to the conclusion that

$$
\eta=\eta_{\mathrm{WMAP}}=(6.19 \pm 0.15) \times 10^{-10} .
$$

This number being fixed, all abundances can be computed. At present, there exists a discrepancy between the predicted abundance of lithium- 7 based on the WMAP results $[108,107]$ for $\eta,{ }^{7} \mathrm{Li} / \mathrm{H}=$ $(5.14 \pm 0.50) \times 10^{-10}$ and its values measured in metal-poor halo stars in our galaxy [63], ${ }^{7} \mathrm{Li} / \mathrm{H}=$ 
$(1.26 \pm 0.26) \times 10^{-10}$, which is a factor of three lower, at least [116] (see also [469]), than the predicted value. No solution to this Lithium-7 problem is known. A back of the envelope estimates shows that we can mimic a lower $\eta$ parameter, just by modifying the deuterium binding energy, letting $T_{N}$ unchanged, since from Equation (123), one just need $\Delta B_{D} / T_{\mathrm{N}} \sim-\ln 9$ so that the effective $\eta$ parameter, assuming no variation of constant, is three times smaller than $\eta_{\text {WMAP }}$. This rough rule of thumb explains that the solution of the lithium-7 problem may lie in a possible variation of the fundamental constants (see below for details).

\subsubsection{Constants everywhere...}

In complete generality, the effect of varying constants on the BBN predictions is difficult to model because of the intricate structure of QCD and its role in low energy nuclear reactions. Thus, a solution is to proceed in two steps, first by determining the dependencies of the light element abundances on the BBN parameters and then by relating those parameters to the fundamental constants.

The analysis of the previous Section 3.8.1, that was restricted to the helium-4 case, clearly shows that the abundances will depend on: (1) $\alpha_{\mathrm{G}}$, which will affect the Hubble expansion rate at the time of nucleosynthesis in the same way as extra-relativistic degrees of freedom do, so that it modifies the freeze-out time $T_{\mathrm{f}}$. This is the only gravitational sector parameter. (2) $\tau_{\mathrm{n}}$, the neutron lifetime dictates the free neutron decay and appears in the normalization of the proton-neutron reaction rates. It is the only weak interaction parameter and it is related to the Fermi constant $G_{\mathrm{F}}$, or equivalently the Higgs vev. (3) $\alpha_{\mathrm{EM}}$, the fine-structure constant. It enters in the Coulomb barriers of the reaction rates through the Gamow factor, in all the binding energies. (4) $Q_{\mathrm{np}}$, the neutron-proton mass difference enters in the neutron-proton ratio and we also have a dependence in (5) $m_{\mathrm{N}}$ and $m_{\mathrm{e}}$ and (6) the binding energies.

Clearly all these parameters are not independent but their relation is often model-dependent. If we focus on helium-4, its abundance mainly depends on $Q_{\mathrm{np}}, T_{\mathrm{f}}$ and $T_{\mathrm{N}}$ (and hence mainly on the neutron lifetime, $\tau_{\mathrm{n}}$ ). Early studies (see Section III.C.2 of FVC [500]) generally focused on one of these parameters. For instance, Kolb et al. [295] calculated the dependence of primordial ${ }^{4} \mathrm{He}$ on $G, G_{\mathrm{F}}$ and $Q_{\mathrm{np}}$ to deduce that the helium- 4 abundance was mostly sensitive in the change in $Q_{\mathrm{np}}$ and that other abundances were less sensitive to the value of $Q_{\mathrm{np}}$, mainly because ${ }^{4} \mathrm{He}$ has a larger binding energy; its abundances is less sensitive to the weak reaction rate and more to the parameters fixing the value of $(n / p)$. To extract the constraint on the fine-structure constant, they decomposed $Q_{\mathrm{np}}$ as $Q_{\mathrm{np}}=\alpha_{\mathrm{EM}} Q_{\alpha}+\beta Q_{\beta}$ where the first term represents the electromagnetic contribution and the second part corresponds to all non-electromagnetic contributions. Assuming that $Q_{\alpha}$ and $Q_{\beta}$ are constant and that the electromagnetic contribution is the dominant part of $Q$, they deduced that $\left|\Delta \alpha_{\mathrm{EM}} / \alpha_{\mathrm{EM}}\right|<10^{-2}$. Campbell and Olive [77] kept track of the changes in $T_{\mathrm{f}}$ and $Q_{\mathrm{np}}$ separately and deduced that $\frac{\Delta Y_{\mathrm{p}}}{Y_{\mathrm{p}}} \simeq \frac{\Delta T_{\mathrm{f}}}{T_{\mathrm{f}}}-\frac{\Delta Q_{\mathrm{np}}}{Q_{\mathrm{np}}}$ while more recently the analysis [308] focused on $\alpha_{\mathrm{EM}}$ and $v$.

Let us now see how the effect of all these parameters are now accounted for in BBN codes.

Bergström et al. [51] started to focus on the $\alpha_{\mathrm{EM}}$-dependence of the thermonuclear rates (see also Ref. [260]). In the non-relativistic limit, it is obtained as the thermal average of the product of the cross, the relative velocity and the the number densities. Charged particles must tunnel through a Coulomb barrier to react. Changing $\alpha_{\mathrm{EM}}$ modifies these barriers and thus the reaction rates. Separating the Coulomb part, the low-energy cross section can be written as

$$
\sigma(E)=\frac{S(E)}{E} \mathrm{e}^{-2 \pi \eta(E)}
$$

where $\eta(E)$ arises from the Coulomb barrier and is given in terms of the charges and the reduced

Living Reviews in Relativity

http://www. livingreviews. org/lrr-2011-2 
mass $M_{r}$ of the two interacting particles as

$$
\eta(E)=\alpha_{\mathrm{EM}} Z_{1} Z_{2} \sqrt{\frac{M_{r} c^{2}}{2 E}} .
$$

The form factor $S(E)$ has to be extrapolated from experimental nuclear data but its $\alpha_{\mathrm{EM}}$-dependence as well as the one of the reduced mass were neglected. Keeping all other constants fixed, assuming no exotic effects and taking a lifetime of $886.7 \mathrm{~s}$ for the neutron, it was deduced that $\left|\Delta \alpha_{\mathrm{EM}} / \alpha_{\mathrm{EM}}\right|<2 \times 10^{-2}$. This analysis was then extended [385] to take into account the $\alpha_{\mathrm{EM}^{-}}$ dependence of the form factor to conclude that

$$
\sigma(E)=\frac{2 \pi \eta(E)}{\exp ^{2 \pi \eta(E)}-1} \simeq 2 \pi \alpha_{\mathrm{EM}} Z_{1} Z_{2} \sqrt{\frac{M_{r} c^{2}}{c^{2}}} \exp ^{-2 \pi \eta(E)} .
$$

Ref. [385] also took into account (1) the effect that when two charged particles are produced they must escape the Coulomb barrier. This effect is generally weak because the $Q_{i}$-values (energy release) of the different reactions are generally larger than the Coulomb barrier at the exception of two cases, ${ }^{3} \mathrm{He}(n, p){ }^{3} \mathrm{H}$ and ${ }^{7} \mathrm{Be}(n, p){ }^{7} \mathrm{Li}$. The rate of these reactions must be multiplied by a factor $\left(1+a_{i} \Delta \alpha_{\mathrm{EM}} / \alpha_{\mathrm{EM}}\right)$. (2) The radiative capture (photon emitting processes) are proportional to $\alpha_{\mathrm{EM}}$ since it is the strength of the coupling of the photon and nuclear currents. All these rates need to be multiplied by $\left(1+\Delta \alpha_{\mathrm{EM}} / \alpha_{\mathrm{EM}}\right)$. (3) The electromagnetic contribution to all masses was taken into account, which modify the $Q_{i}$-values as $\left.Q_{i} \rightarrow Q_{i}+q_{i} \Delta \alpha_{\mathrm{EM}} / \alpha_{\mathrm{EM}}\right)$. For helium- 4 abundance these effects are negligible since the main $\alpha_{\mathrm{EM}}$-dependence arises from $Q_{\mathrm{np}}$. Equipped with these modifications, it was concluded that $\Delta \alpha_{\mathrm{EM}} / \alpha_{\mathrm{EM}}=-0.007_{-0.017}^{+0.010}$ using only deuterium and helium-4 since the lithium-7 problem was still present.

Then the focus fell on the deuterium binding energy, $B_{D}$. Flambaum and Shuryak [207, 208, $158,157]$ illustrated the sensitivity of the light element abundances on $B_{D}$. Its value mainly sets the beginning of the nucleosynthesis, that is of $T_{\mathrm{N}}$ since the temperature must low-enough in order for the photo-dissociation of the deuterium to be negligible (this is at the origin of the deuterium bottleneck). The importance of $B_{D}$ is easily understood by the fact that the equilibrium abundance of deuterium and the reaction rate $p(n, \gamma) \mathrm{D}$ depends exponentially on $B_{D}$ and on the fact that the deuterium is in a shallow bound state. Focusing on the $T_{\mathrm{N}}$-dependence, it was concluded [207] that $\Delta B_{D} / B_{D}<0.075$.

This shows that the situation is more complex and that one cannot reduce the analysis to a single varying parameter. Many studies then tried to determinate the sensitivity to the variation of many independent parameters.

The sensitivity of the helium- 4 abundance to the variation of 7 parameters was first investigated by Müller et al. [364] considering the dependence on the parameters $\left\{X_{i}\right\} \equiv\left\{G, \alpha_{\mathrm{EM}}, v, m_{\mathrm{e}}, \tau_{\mathrm{n}}, Q_{\mathrm{np}}\right.$, $\left.B_{D}\right\}$ independently,

$$
\Delta \ln Y_{\mathrm{He}}=\sum_{i} c_{i}^{(X)} \Delta \ln X_{i}
$$

and assuming $\Lambda_{\mathrm{QCD}}$ fixed (so that the seven parameters are in fact dimensionless quantities). The $c_{i}^{(X)}$ are the sensitivities to the BBN parameters, assuming the six others are fixed. It was concluded that $Y_{\mathrm{He}} \propto \alpha_{\mathrm{EM}}^{-0.043} v^{2.4} m_{\mathrm{e}}^{0.024} \tau_{\mathrm{n}}^{0.24} Q_{\mathrm{np}}^{-1.8} B_{D}^{0.53} G^{0.405}$ for independent variations. They further related $\left(\tau_{\mathrm{n}}, Q_{\mathrm{np}}, B_{D}\right)$ to $\left(\alpha_{\mathrm{EM}}, v, m_{\mathrm{e}}, m_{\mathrm{N}}, m_{\mathrm{d}}-m_{\mathrm{u}}\right)$, as we shall discuss in the next Section 3.8.3.

This was generalized by Landau et al. [309] up to lithium-7 considering the parameters $\left\{\alpha_{\mathrm{EM}}, G_{\mathrm{F}}\right.$, $\left.\Lambda_{\mathrm{QCD}}, \Omega_{b} h^{2}\right\}$, assuming $G$ constant where the variation of $\tau_{\mathrm{n}}$ and the variation of the masses where tied to these parameters but the effect on the binding energies were not considered.

Coc et al. [104] considered the effect of a variation of $\left(Q_{\mathrm{np}}, B_{D}, \tau_{\mathrm{n}}, m_{\mathrm{e}}\right)$ on the abundances of the light elements up to lithium-7, neglecting the effect of $\alpha_{\mathrm{EM}}$ on the . Their dependence on the 

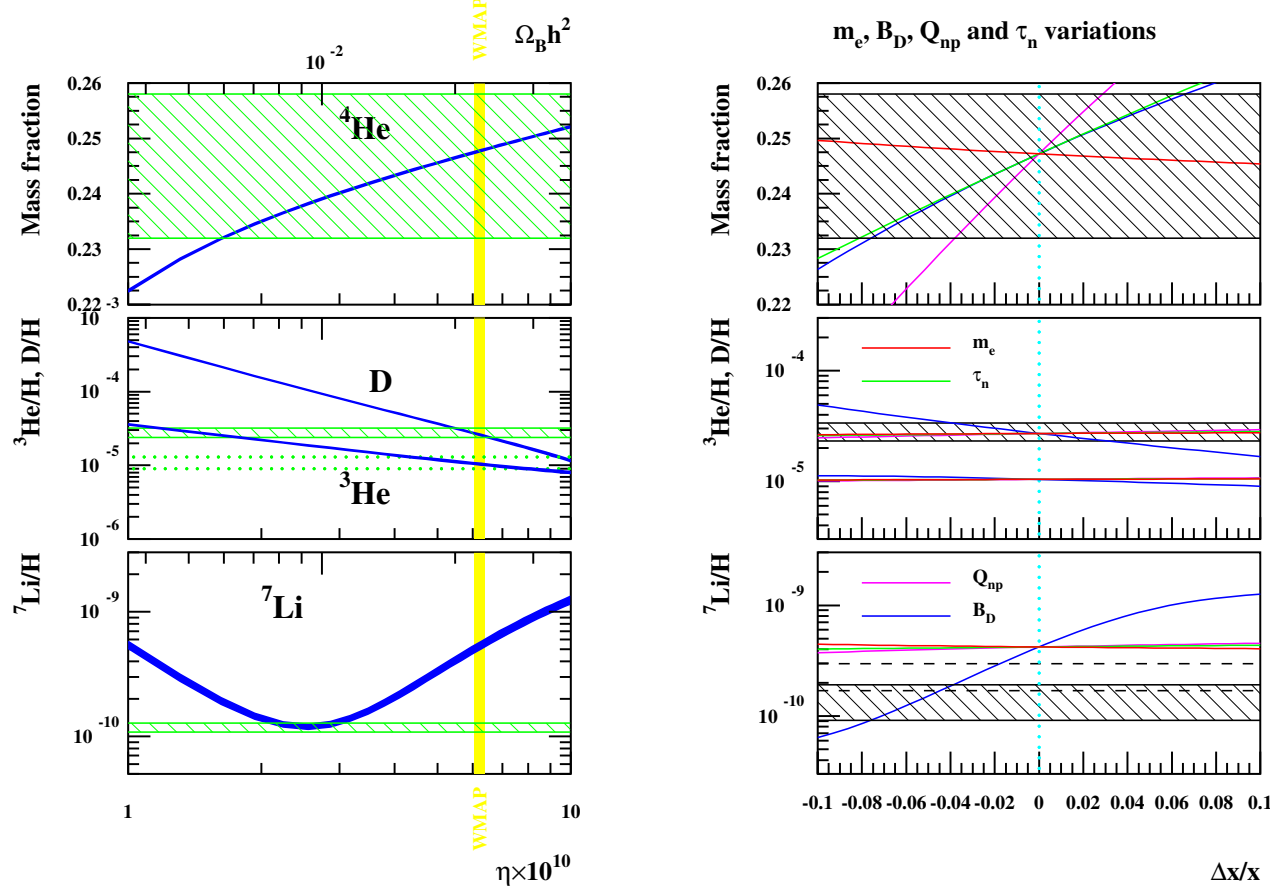

Figure 6: (Left): variation of the light element abundances in function of $\eta$ compared to the spectroscopic abundances. The vertical line depicts the constraint obtained on $\eta$ from the study of the cosmic microwave background data. The lithium- 7 problem lies in the fact that $\eta_{\text {spectro }}<\eta_{\text {WMAP }}$. From [107]. (right): Dependence of the light element abundance on the independent variation of the BBN parameters, assuming $\eta=\eta_{\mathrm{WMAP}}$. From [105] 
independent variation of each of these parameters is depicted on Figure 6. It confirmed the result of $[207,394]$ that the deuterium binding energy is the most sensitive parameter. From the helium- 4 data alone, the bounds

$$
-8.2 \times 10^{-2} \lesssim \frac{\Delta \tau_{\mathrm{n}}}{\tau_{\mathrm{n}}} \lesssim 6 \times 10^{-2}, \quad-4 \times 10^{-2} \lesssim \frac{\Delta Q_{\mathrm{np}}}{Q_{\mathrm{np}}} \lesssim 2.7 \times 10^{-2}
$$

and

$$
-7.5 \times 10^{-2} \lesssim \frac{\Delta B_{D}}{B_{D}} \lesssim 6.5 \times 10^{-2}
$$

at a $2 \sigma$ level, were set (assuming $\eta_{\text {WMAP }}$ ). The deuterium data set the tighter constraint $-4 \times$ $10^{-2} \lesssim \Delta \ln B_{D} \lesssim 3 \times 10^{-2}$. Note also on Figure 6 that the lithium-7 abundance can be brought in concordance with the spectroscopic observations provided that $B_{D}$ was smaller during $\mathrm{BBN}$

$$
-7.5 \times 10^{-2} \lesssim \frac{\Delta B_{D}}{B_{D}} \lesssim-4 \times 10^{-2}
$$

so that $B_{D}$ may be the most important parameter to resolve the lithium-7 problem. The effect of the quark mass on the binding energies was described in [49]. They then concluded that a variation of $\Delta m_{\mathrm{q}} / m_{\mathrm{q}}=0.013 \pm 0.002$ allows to reconcile the abundance of lithium- 7 and the value of $\eta$ deduced from WMAP.

This analysis was extended [146] to incorporate the effect of 13 independent BBN parameters including the parameters considered before plus the binding energies of deuterium, tritium, helium3 , helium-4, lithium-6, lithium-7 and beryllium-7. The sensitivity of the light element abundances to the independent variation of these parameters is summarized in Table I of [146]. These BBN parameters were then related to the same 6 "fundamental" parameters used in [364].

All these analyses demonstrate that the effects of the BBN parameters on the light element abundances are now under control. They have been implemented in BBN codes and most results agree, as well as with semi-analytical estimates. As long as these parameters are assume to vary independently, no constraints sharper than $10^{-2}$ can be set. One should also not forget to take into account standard parameters of the BBN computation such as $\eta$ and the effective number of relativistic particle.

\subsubsection{From BBN parameters to fundamental constants}

To reduce the number parameters, we need to relate the BBN parameters to more fundamental ones, keeping in mind that this can usually be done only in a model-dependent way. We shall describe some of the relations that have been used in many studies. They mainly concern $Q_{\mathrm{np}}, \tau_{\mathrm{n}}$ and $B_{D}$.

At lowest order, all dimensional parameters of QCD, e.g., masses, nuclear energies etc., are to a good approximation simply proportional to some powers of $\Lambda_{\mathrm{QCD}}$. One needs to go beyond such a description and takes the effects of the masses of the quarks into account.

$Q_{\mathrm{np}}$ can be expressed in terms of the mass on the quarks $\mathrm{u}$ and $\mathrm{d}$ and the fine-structure constant as

$$
Q_{\mathrm{np}}=a \alpha_{\mathrm{EM}} \Lambda_{\mathrm{QCD}}+\left(m_{\mathrm{d}}-m_{\mathrm{u}}\right),
$$

where the electromagnetic contribution today is $\left(a \alpha_{\mathrm{EM}} \Lambda_{\mathrm{QCD}}\right)_{0}=-0.76 \mathrm{MeV}$ and therefore the quark mass contribution today is $\left(m_{\mathrm{d}}-m_{\mathrm{u}}\right)=2.05$ [230] so that

$$
\frac{\Delta Q_{\mathrm{np}}}{Q_{\mathrm{np}}}=-0.59 \frac{\Delta \alpha_{\mathrm{EM}}}{\alpha_{\mathrm{EM}}}+1.59 \frac{\Delta\left(m_{\mathrm{d}}-m_{\mathrm{u}}\right)}{\left(m_{\mathrm{d}}-m_{\mathrm{u}}\right)} .
$$

All the analyses cited above agree on this dependence. 
The neutron lifetime can be well approximated by

$$
\tau_{\mathrm{n}}^{-1}=\frac{1+3 g_{A}^{2}}{120 \pi^{3}} G_{\mathrm{F}}^{2} m_{\mathrm{e}}^{5}\left[\sqrt{q^{2}-1}\left(2 q^{4}-9 q^{2}-8\right)+15 \ln \left(q+\sqrt{q^{2}-1}\right)\right]
$$

with $q \equiv Q_{\mathrm{np}} / m_{\mathrm{e}}$ and $G_{\mathrm{F}}=1 / \sqrt{2} v^{2}$. Using the former expression for $Q_{\mathrm{np}}$ we can express $\tau_{\mathrm{n}}$ in terms of $\alpha_{\mathrm{EM}}, v$ and the $\mathrm{u}, \mathrm{d}$ and electron masses. It follows

$$
\frac{\Delta \tau_{\mathrm{n}}}{\tau_{\mathrm{n}}}=3.86 \frac{\Delta \alpha_{\mathrm{EM}}}{\alpha_{\mathrm{EM}}}+4 \frac{\Delta v}{v}+1.52 \frac{\Delta m_{\mathrm{e}}}{m_{\mathrm{e}}}-10.4 \frac{\Delta\left(m_{\mathrm{d}}-m_{\mathrm{u}}\right)}{\left(m_{\mathrm{d}}-m_{\mathrm{u}}\right)} .
$$

Again, all the analyses cited above agree on this dependence.

Let us now turn to the binding energies, and more particularly to $B_{D}$ that, as we have seen, is a crucial parameter. This is one the better known quantities in the nuclear domain and it is experimentally measured to a precision better than $10^{-6}[19]$. Two approaches have been followed.

- Pion mass. A first route is to use the dependence of the binding energy on the pion mass [188, 38], which is related to the $\mathrm{u}$ and d quark masses by

$$
m_{\pi}^{2}=m_{\mathrm{q}}\langle\bar{u} u+\bar{d} d\rangle f_{\pi}^{-2} \simeq \hat{m} \Lambda_{\mathrm{QCD}}
$$

where $m_{\mathrm{q}} \equiv \frac{1}{2}\left(m_{\mathrm{u}}+m_{\mathrm{d}}\right)$ and assuming that the leading order of $\langle\bar{u} u+\bar{d} d\rangle f_{\pi}^{-2}$ depends only on $\Lambda_{\mathrm{QCD}}, f_{\pi}$ being the pion decay constant. This dependence was parameterized [553] as

$$
\frac{\Delta B_{D}}{B_{D}}=-r \frac{\Delta m_{\pi}}{m_{\pi}}
$$

where $r$ is a fitting parameter found to be between 6 [188] and 10 [38]. Prior to this result, the analysis of [207] provides two computations of this dependence, which respectively lead to $r=-3$ and $r=18$ while, following the same lines, [88] got $r=0.082$.

[364], following the computations of [426], adds an electromagnetic contribution $-0.0081 \Delta \alpha_{\mathrm{EM}} / \alpha_{\mathrm{EM}}$ so that

$$
\frac{\Delta B_{D}}{B_{D}}=-\frac{r}{2} \frac{\Delta m_{\mathrm{q}}}{m_{\mathrm{q}}}-0.0081 \frac{\Delta \alpha_{\mathrm{EM}}}{\alpha_{\mathrm{EM}}}
$$

but this latter contribution has not been included in other work.

- Sigma model. In the framework of the Walecka model, where the potential for the nuclear forces keeps only the $\sigma$ and $\omega$ meson exchanges,

$$
V=-\frac{g_{s}^{2}}{4 \pi r} \exp \left(-m_{\sigma} r\right)+\frac{g_{v}^{2}}{4 \pi r} \exp \left(-m_{\omega} r\right)
$$

where $g_{s}$ and $g_{v}$ are two coupling constants. Describing $\sigma$ as a $\mathrm{SU}(3)$ singlet state, its mass was related to the mass of the strange quark. In this way one can hope to take into account the effect of the strange quark, both on the nucleon mass and the binding energy. In a second step $B_{D}$ is related to the meson and nucleon mass by

$$
\frac{\Delta B_{D}}{B_{D}}=-48 \frac{\Delta m_{\sigma}}{m_{\sigma}}+50 \frac{\Delta m_{\omega}}{m_{\omega}}+6 \frac{\Delta m_{\mathrm{N}}}{m_{\mathrm{N}}}
$$

so that $\Delta B_{D} / B_{D} \simeq-17 \Delta m_{\mathrm{s}} m_{\mathrm{s}}$ [208]. Unfortunately, a complete treatment of all the nuclear quantities on $m_{\mathrm{s}}$ has not been performed yet. 
The case of the binding energies of the other elements has been less studied. [146] follows a route similar than for $B_{D}$ and relates them to pion mass and assumes that

$$
\frac{\partial B_{i}}{\partial m_{\pi}}=f_{i}\left(A_{i}-1\right) \frac{B_{D}}{m_{\pi}} r \simeq-0.13 f_{i}\left(A_{i}-1\right),
$$

where $f_{i}$ are unknown coefficients assumed to be of order unity and $A_{i}$ is the number of nucleons. No other estimates has been performed. Other nuclear potentials (such as Reid 93 potential, Nijmegen potential, Argonne $v 18$ potential and Bonn potential) have been used in [101] to determine the dependence of $B_{D}$ on $v$ and agree with previous studies.

These analyses allow one to reduce all the BBN parameter to the physical constants $\left(\alpha_{\mathrm{EM}}, v\right.$, $\left.m_{\mathrm{e}}, m_{\mathrm{d}}-m_{\mathrm{u}}, m_{\mathrm{q}}\right)$ and $G$ that is not affected by this discussion. This set can be further reduced, since all the masses can be expressed in terms of $v$ as $m_{i}=h_{i} v$, where $h_{i}$ are Yukawa couplings.

To go further, one needs to make more assumption, such as grand unification, or by relating the Yukawa coupling of the top to $v$ by assuming that weak scale is determined by dimensional transmutation [104], or that the variation of the constant is induced by a string dilaton [77]. At each step, one gets more stringent constraints, which can reach the $10^{-4}$ [146] to $10^{-5}$ [104] level but indeed more model-dependent!

\subsubsection{Conclusion}

Primordial nucleosynthesis offers a possibility to test almost all fundamental constants of physics at a redshift of $z \sim 10^{8}$. This makes it very rich but indeed the effect of each constant is more difficult to disentangle. The effect of the BBN parameters has been quantified with precision and they can be constrained typically at a $10^{-2}$ level, and in particular it seems that the most sensitive parameter is the deuterium binding energy.

The link with more fundamental parameters is better understood but the dependence of the deuterium binding energy still left some uncertainties and a good description of the effect of the strange quark mass is missing.

We have not considered the variation of $G$ in this section. Its effect is disconnected from the other parameters. Let us just stress that assuming the BBN sensitivity on $G$ by just modifying its value may be misleading. In particular $G$ can vary a lot during the electron-positron annihilation so that the BBN constraints can in general not be described by an effective speed-up factor $[105,134]$. 


\section{The Gravitational Constant}

The gravitational constant was the first constant whose constancy was questioned [155]. From a theoretical point of view, theories with a varying gravitational constant can be designed to satisfy the equivalence principle in its weak form but not in its strong form [540] (see also Section 5). Most theories of gravity that violate the strong equivalence principle predict that the locally measured gravitational constant may vary with time.

The value of the gravitational constant is $G=6.67428(67) \times 10^{-11} \mathrm{~m}^{3} \mathrm{~kg}^{-1} \mathrm{~s}^{-2}$ so that its relative standard uncertainty fixed by the $\mathrm{CODATA}^{11}$ in 2006 is $0.01 \%$. Interestingly, the disparity between different experiments led, in 1998, to a temporary increase of this uncertainty to $0.15 \%$ [241], which demonstrates the difficulty in measuring the value of this constant. This explains partly why the constraints on the time variation are less stringent than for the other constants.

A variation of the gravitational constant, being a pure gravitational phenomenon, does not affect the local physics, such as, e.g., the atomic transitions or the nuclear physics. In particular, it is equivalent at stating that the masses of all particles are varying in the same way to that their ratios remain constant. Similarly all absorption lines will be shifted in the same way. It follows that most constraints are obtained from systems in which gravity is non-negligible, such as the motion of the bodies of the Solar system, astrophysical and cosmological systems. They are mostly related in the comparison of a gravitational time scale, e.g., period of orbits, to a non-gravitational time scale. It follows that in general the constraints assume that the values of the other constants are fixed. Taking their variation into account would add degeneracies and make the constraints cited below less stringent.

We refer to Section IV of FVC [500] for earlier constraints based, e.g., on the determination of the Earth surface temperature, which roughly scales as $G^{2.25} M_{\odot}^{1.75}$ and gives a constraint of the order of $|\Delta G / G|<0.1$ [224], or on the estimation of the Earth radius at different geological epochs. We also emphasize that constraints on the variation of $G$ are meant to be constraints on the dimensionless parameter $\alpha_{\mathrm{G}}$.

\subsection{Solar systems constraints}

Monitoring the orbits of the various bodies of the solar system offers a possibility to constrain deviations from general relativity, and in particular the time variation of $G$. This accounts for comparing a gravitational time scale (related to the orbital motion) and an atomic time scale and it is thus assumed that the variation of atomic constants is negligible over the time of the experiment.

The first constraint arises from the Earth-Moon system. A time variation of $G$ is then related to a variation of the mean motion $(n=2 \pi / P)$ of the orbit of the Moon around the Earth. A decrease in $G$ would induce both the Lunar mean distance and period to increase. As long as the gravitational binding energy is negligible, one has

$$
\frac{\dot{P}}{P}=-2 \frac{\dot{G}}{G}
$$

Earlier constraints rely on paleontological data and ancient eclipses observations (see Section IV.B.1 of FVC [500]) and none of them are very reliable. A main difficulty arises from tidal dissipation that also causes the mean distance and orbital period to increase (for tidal changes $2 \dot{n} / n+3 \dot{a} / a=0$ ), but not as in the same ratio as for $\dot{G}$.

The Lunar Laser Ranging (LLR) experiment has measured the relative position of the Moon with respect to the Earth with an accuracy of the order of $1 \mathrm{~cm}$ over 3 decades. An early analysis

11 The CODATA is the COmmittee on Data for Science and Technology, see http://www. codata.org/.

Living Reviews in Relativity

http: //www . livingreviews . org/lrr-2011-2 
of this data [544] assuming a Brans-Dicke theory of gravitation gave that $|\dot{G} / G| \leq 3 \times 10^{-11} \mathrm{yr}^{-1}$. It was improved [365] by using 20 years of observation to get $|\dot{G} / G| \leq 1.04 \times 10^{-11} \mathrm{yr}^{-1}$, the main uncertainty arising from Lunar tidal acceleration. With, 24 years of data, one reached [542] $|\dot{G} / G| \leq 6 \times 10^{-12} \mathrm{yr}^{-1}$ and finally, the latest analysis of the Lunar laser ranging experiment [543] increased the constraint to

$$
\left.\frac{\dot{G}}{G}\right|_{0}=(4 \pm 9) \times 10^{-13} \mathrm{yr}^{-1} .
$$

Similarly, Shapiro et al. [458] compared radar-echo time delays between Earth, Venus and Mercury with a caesium atomic clock between 1964 and 1969. The data were fitted to the theoretical equation of motion for the bodies in a Schwarzschild spacetime, taking into account the perturbations from the Moon and other planets. They concluded that $|\dot{G} / G|<4 \times 10^{-10} \mathrm{yr}^{-1}$. The data concerning Venus cannot be used due to imprecision in the determination of the portion of the planet reflecting the radar. This was improved to $|\dot{G} / G|<1.5 \times 10^{-10} \mathrm{yr}^{-1}$ by including Mariner 9 and Mars orbiter data [429]. The analysis was further extended [457] to give $\dot{G} / G=(-2 \pm 10) \times 10^{-12} \mathrm{yr}^{-1}$. The combination of Mariner 10 an Mercury and Venus ranging data gives [12]

$$
\left.\frac{\dot{G}}{G}\right|_{0}=(0.0 \pm 2.0) \times 10^{-12} \mathrm{yr}^{-1} .
$$

Reasenberg et al. [430] considered the 14 months data obtained from the ranging of the Viking spacecraft and deduced, assuming a Brans-Dicke theory, $|\dot{G} / G|<10^{-12} \mathrm{yr}^{-1}$. Hellings et al. [249] using all available astrometric data and in particular the ranging data from Viking landers on Mars deduced that

$$
\left.\frac{\dot{G}}{G}\right|_{0}=(2 \pm 4) \times 10^{-12} \mathrm{yr}^{-1} .
$$

The major contribution to the uncertainty is due to the modeling of the dynamics of the asteroids on the Earth-Mars range. Hellings et al. [249] also tried to attribute their result to a time variation of the atomic constants. Using the same data but a different modeling of the asteroids, Reasenberg [428] got $|\dot{G} / G|<3 \times 10^{-11} \mathrm{yr}^{-1}$, which was then improved by Chandler et al. [93] to $|\dot{G} / G|<10^{-11} \mathrm{yr}^{-1}$.

\subsection{Pulsar timing}

Contrary to the solar system case, the dependence of the gravitational binding energy cannot be neglected while computing the time variation of the period. Here two approaches can be followed; either one sticks to a model (e.g., scalar-tensor gravity) and compute all the effects in this model or one has a more phenomenological approach and tries to put some model-independent bounds.

Eardley [177] followed the first route and discussed the effects of a time variation of the gravitational constant on binary pulsar in the framework of the Brans-Dicke theory. In that case, both a dipole gravitational radiation and the variation of $G$ induce a periodic variation in the pulse period. Nordtvedt [386] showed that the orbital period changes as

$$
\frac{\dot{P}}{P}=-\left[2+\frac{2\left(m_{1} c_{1}+m_{2} c_{2}\right)+3\left(m_{1} c_{2}+m_{2} c_{1}\right)}{m_{1}+m_{2}}\right] \frac{\dot{G}}{G}
$$

where $c_{i} \equiv \delta \ln m_{i} / \delta \ln G$. He concluded that for the pulsar PSR 1913+16 $\left(m_{1} \simeq m_{2}\right.$ and $\left.c_{1} \simeq c_{2}\right)$ one gets

$$
\frac{\dot{P}}{P}=-[2+5 c] \frac{\dot{G}}{G}
$$


the coefficient $c$ being model dependent. As another application, he estimated that $c_{\text {Earth }} \sim$ $-5 \times 10^{-10}, c_{\text {Moon }} \sim-10^{-8}$ and $c_{\text {Sun }} \sim-4 \times 10^{-6}$ justifying the formula used in the solar system.

Damour et al. [127] used the timing data of the binary pulsar PSR $1913+16$. They implemented the effect of the time variation of $G$ by considering the effect on $\dot{P} / P$. They defined, in a phenomenological way, that $\dot{G} / G=-0.5 \delta \dot{P} / P$, where $\delta \dot{P}$ is the part of the orbital period derivative that is not explained otherwise (by gravitational waves radiation damping). This theory-independent definition has to be contrasted with the theory-dependent result (138) by Nordtvedt [386]. They got

$$
\dot{G} / G=(1.0 \pm 2.3) \times 10^{-11} \mathrm{yr}^{-1} .
$$

Damour and Taylor [137] then reexamined the data of PSR 1913+16 and established the upper bound

$$
\dot{G} / G<(1.10 \pm 1.07) \times 10^{-11} \mathrm{yr}^{-1} .
$$

Kaspi et al. [282] used data from PSR B1913+16 and PSR B1855+09 respectively to get

$$
\dot{G} / G=(4 \pm 5) \times 10^{-12} \mathrm{yr}^{-1}
$$

and

$$
\dot{G} / G=(-9 \pm 18) \times 10^{-12} \mathrm{yr}^{-1},
$$

the latter case being more "secure" since the orbiting companion is not a neutron star.

All the previous results concern binary pulsars but isolated ones can also be used. Heintzmann and Hillebrandt [248] related the spin-down of the pulsar JP1953 to a time variation of $G$. The spin-down is a combined effect of electromagnetic losses, emission of gravitational waves, possible spin-up due to matter accretion. Assuming that the angular momentum is conserved so that $I / P=$ constant, one deduces that

$$
\left.\frac{\dot{P}}{P}\right|_{G}=\left(\frac{\mathrm{d} \ln I}{\mathrm{~d} \ln G}\right) \frac{\dot{G}}{G}
$$

The observational spin-down can be decomposed as

$$
\left.\frac{\dot{P}}{P}\right|_{\mathrm{obs}}=\left.\frac{\dot{P}}{P}\right|_{\mathrm{mag}}+\left.\frac{\dot{P}}{P}\right|_{\mathrm{GW}}+\left.\frac{\dot{P}}{P}\right|_{G} .
$$

Since $\dot{P} / P_{\text {mag }}$ and $\dot{P} / P_{\mathrm{GW}}$ are positive definite, it follows that $\dot{P} / P_{\text {obs }} \geq \dot{P} / P_{G}$ so that a bound on $\dot{G}$ can be inferred if the main pulse period is the period of rotation. Heintzmann and Hillebrandt [248] then modelled the pulsar by a polytropic $\left(P \propto \rho^{n}\right)$ white dwarf and deduced that $\mathrm{d} \ln I / \mathrm{d} \ln G=2-3 n / 2$ so that $|\dot{G} / G|<10^{-10} \mathrm{yr}^{-1}$. Mansfield [344] assumed a relativistic degenerate, zero temperature polytropic star and got that, when $\dot{G}<0,0 \leq-\dot{G} / G<6.8 \times 10^{-11} \mathrm{yr}^{-1}$ at a $2 \sigma$ level. He also noted that a positive $\dot{G}$ induces a spin-up counteracting the electromagnetic spin-down, which can provide another bound if an independent estimate of the pulsar magnetic field can be obtained. Goldman [233], following Eardley [177], used the scaling relations $N \propto G^{-3 / 2}$ and $M \propto G^{-5 / 2}$ to deduce that $2 \mathrm{~d} \ln I / \mathrm{d} \ln G=-5+3 \mathrm{~d} \ln I / \mathrm{d} \ln N$. He used the data from the pulsar PSR $0655+64$ to deduce that the rate of decrease of $G$ was smaller than

$$
0 \leq-\dot{G} / G<5.5 \times 10^{-11} \mathrm{yr}^{-1} .
$$

The analysis [516] of 10 years high precision timing data on the millisecond pulsar PSR J0437-4715 has allowed to improve the constraint to

$$
|\dot{G} / G|<2.3 \times 10^{-11} \mathrm{yr}^{-1} \text {. }
$$


Recently, it was argued $[266,432]$ that a variation of $G$ would induce a departure of the neutron star matter from $\beta$-equilibrium, due to the changing hydrostatic equilibrium. This would force non-equilibrium $\beta$-processes to occur, which release energy that is invested partly in neutrino emission and partly in heating the stellar interior. Eventually, the star arrives at a stationary state in which the temperature remains nearly constant, as the forcing through the change of $G$ is balanced by the ongoing reactions. Comparing the surface temperature of the nearest millisecond pulsar, PSR J0437-4715, inferred from ultraviolet observations, two upper limits for variation were obtained, $|\dot{G} / G|<2 \times 10^{-10} \mathrm{yr}^{-1}$, direct Urca reactions operating in the neutron star core are allowed, and $|\dot{G} / G|<4 \times 10^{-12} \mathrm{yr}^{-1}$, considering only modified Urca reactions. This was extended in [302] in order to take into account the correlation between the surface temperatures and the radii of some old neutron stars to get $|\dot{G} / G|<2.1 \times 10^{-11} \mathrm{yr}^{-1}$.

\subsection{Stellar constraints}

Early works, see Section IV.C of FVC [500], studied the Solar evolution in presence of a time varying gravitational constant, concluding that under the Dirac hypothesis, the original nuclear resources of the Sun would have been burned by now. This results from the fact that an increase of the gravitational constant is equivalent to an increase of the star density (because of the Poisson equation).

The idea of using stellar evolution to constrain the possible value of $G$ was originally proposed by Teller [487], who stressed that the evolution of a star was strongly dependent on $G$. The luminosity of a main sequence star can be expressed as a function of Newton's gravitational constant and its mass by using homology relations [224, 487]. In the particular case that the opacity is dominated by free-free transitions, Gamow [224] found that the luminosity of the star is given approximately by $L \propto G^{7.8} M^{5.5}$. In the case of the Sun, this would mean that for higher values of $G$, the burning of hydrogen will be more efficient and the star evolves more rapidly, therefore we need to increase the initial content of hydrogen to obtain the present observed Sun. In a numerical test of the previous expression, Delg'Innocenti et al. [140] found that low-mass stars evolving from the Zero Age Main Sequence to the red giant branch satisfy $L \propto G^{5.6} M^{4.7}$, which agrees to within $10 \%$ of the numerical results, following the idea that Thomson scattering contributes significantly to the opacity inside such stars. Indeed, in the case of the opacity being dominated by pure Thomson scattering, the luminosity of the star is given by $L \propto G^{4} M^{3}$. It follows from the previous analysis that the evolution of the star on the main sequence is highly sensitive to the value of $G$.

The driving idea behind the stellar constraints is that a secular variation of $G$ leads to a variation of the gravitational interaction. This would affect the hydrostatic equilibrium of the star and in particular its pressure profile. In the case of non-degenerate stars, the temperature, being the only control parameter, will adjust to compensate the modification of the intensity of the gravity. It will then affect the nuclear reaction rates, which are very sensitive to the temperature, and thus the nuclear time scales associated to the various processes. It follows that the main stage of the stellar evolution, and in particular the lifetimes of the various stars, will be modified. As we shall see, basically two types of methods have been used, the first in which on relate the variation of $G$ to some physical characteristic of a star (luminosity, effective temperature, radius), and a second in which only a statistical measurement of the change of $G$ can be inferred. Indeed, the first class of methods are more reliable and robust but is usually restricted to nearby stars. Note also that they usually require to have a precise distance determination of the star, which may depend on $G$.

\subsubsection{Ages of globular clusters}

The first application of these idea has been performed with globular clusters. Their ages, determined for instance from the luminosity of the main-sequence turn-off, have to be compatible with 
the estimation of the age of the galaxy. This gives the constraint [140]

$$
\dot{G} / G=(-1.4 \pm 2.1) \times 10^{-11} \mathrm{yr}^{-1} .
$$

The effect of a possible time dependence of $G$ on luminosity has been studied in the case of globular cluster H-R diagrams but has not yielded any stronger constraints than those relying on celestial mechanics

\subsubsection{Solar and stellar seismology}

A side effect of the change of luminosity is a change in the depth of the convection zone so that the inner edge of the convecting zone changes its location. This induces a modification of the vibration modes of the star and particularly to the acoustic waves, i.e., $p$-modes [141].

Helioseismology. These waves are observed for our star, the Sun, and helioseismology allows one to determine the sound speed in the core of the Sun and, together with an equation of state, the central densities and abundances of helium and hydrogen. Demarque et al. [141] considered an ansatz in which $G \propto t^{-\beta}$ and showed that $|\beta|<0.1$ over the last $4.5 \times 10^{9}$ years, which corresponds to $|\dot{G} / G|<2 \times 10^{-11} \mathrm{yr}^{-1}$. Guenther et al. [240] also showed that $g$-modes could provide even much tighter constraints but these modes are up to now very difficult to observe. Nevertheless, they concluded, using the claim of detection by Hill and Gu [251], that $|\dot{G} / G|<$ $4.5 \times 10^{-12} \mathrm{yr}^{-1}$. Guenther et al. [239] then compared the $p$-mode spectra predicted by different theories with varying gravitational constant to the observed spectrum obtained by a network of six telescopes and deduced that

$$
|\dot{G} / G|<1.6 \times 10^{-12} \mathrm{yr}^{-1} .
$$

The standard solar model depends on few parameters and $G$ plays a important role since stellar evolution is dictated by the balance between gravitation and other interactions. Astronomical observations determines $G M_{\odot}$ with an accuracy better than $10^{-7}$ and a variation of $G$ with $G M_{\odot}$ fixed induces a change of the pressure $\left(P=G M_{\odot}^{2} / R_{\odot}^{2}\right)$ and density $\left(\rho=M_{\odot} / R_{\odot}^{3}\right)$. The experimental uncertainties in $G$ between different experiments have important implications for helioseismology. In particular the uncertainties for the standard solar model lead to a range in the value of the sound speed in the nuclear region that is as much as $0.15 \%$ higher than the inverted helioseismic sound speed [335]. While a lower value of $G$ is preferred for the standard model, any definite prediction is masked by the uncertainties in the solar models available in the literature. Ricci and Villante [436] studied the effect of a variation of $G$ on the density and pressure profile of the Sun and concluded that present data cannot constrain $G$ better than $10^{-2} \%$. It was also shown [335] that the information provided by the neutrino experiments is quite significant because it constitutes an independent test of $G$ complementary to the one provided by helioseismology.

White dwarfs. The observation of the period of non-radial pulsations of white dwarf allows to set similar constraints. White dwarfs represent the final stage of the stellar evolution for stars with a mass smaller to about $10 M_{\odot}$. Their structure is supported against gravitational collapse by the pressure of degenerate electrons. It was discovered that some white dwarfs are variable stars and in fact non-radial pulsator. This opens the way to use seismological techniques to investigate their internal properties. In particular, their non-radial oscillations is mostly determined by the Brunt-Väisälä frequency

$$
N^{2}=g \frac{\mathrm{d} \ln P^{1 / \gamma_{1}} / \rho}{\mathrm{d} r}
$$

where $g$ is the gravitational acceleration, $\Gamma_{1}$ the first adiabatic exponent and $P$ and $\rho$ the pressure and density (see, e.g., [283] for a white dwarf model taking into account a varying $G$ ). A variation of $G$ induces a modification of the degree of degeneracy of the white dwarf, hence on the frequency $N$

Living Reviews in Relativity

http://www. livingreviews.org/lrr-2011-2 
as well as the cooling rate of the star, even though this is thought to be negligible at the luminosities where white dwarfs are pulsationally unstable[54]. Using the observation of G117-B15A that has been monitored during 20 years, it was concluded [43] that

$$
-2.5 \times 10^{-10} \mathrm{yr}^{-1}<\dot{G} / G<4.0 \times 10^{-11} \mathrm{yr}^{-1},
$$

at a $2 \sigma$-level. The same observations were reanalyzed in [54] to obtain

$$
|\dot{G} / G|<4.1 \times 10^{-11} \mathrm{yr}^{-1} .
$$

\subsubsection{Late stages of stellar evolution and supernovae}

A variation of $G$ can influence the white dwarf cooling and the light curves ot Type Ia supernovae.

García-Berro et al. [225] considered the effect of a variation of the gravitational constant on the cooling of white dwarfs and on their luminosity function. As first pointed out by Vila [518], the energy of white dwarfs, when they are cool enough, is entirely of gravitational and thermal origin so that a variation of $G$ will induce a modification of their energy balance and thus of their luminosity. Restricting to cold white dwarfs with luminosity smaller than ten solar luminosity, the luminosity can be related to the star binding energy $B$ and gravitational energy, $E_{\text {grav }}$, as

$$
L=-\frac{\mathrm{d} B}{\mathrm{~d} t}+\frac{\dot{G}}{G} E_{\text {grav }},
$$

which simply results from the hydrostatic equilibrium. Again, the variation of the gravitational constant intervenes via the Poisson equation and the gravitational potential. The cooling process is accelerated if $\dot{G} / G<0$, which then induces a shift in the position of the cut-off in the luminosity function. García-Berro et al. [225] concluded that

$$
0 \leq-\dot{G} / G<(1 \pm 1) \times 10^{-11} \mathrm{yr}^{-1} .
$$

The result depends on the details of the cooling theory, on whether the $\mathrm{C} / \mathrm{O}$ white dwarf is stratified or not and on hypothesis on the age of the galactic disk. For instance, with no stratification of the C/O binary mixture, one would require $\dot{G} / G=-(2.5 \pm 0.5) \times 10^{-11} \mathrm{yr}^{-1}$ if the solar neighborhood has a value of 8 Gyr (i.e., one would require a variation of $G$ to explain the data). In the case of the standard hypothesis of an age of $11 \mathrm{Gyr}$, one obtains that $0 \leq-\dot{G} / G<3 \times 10^{-11} \mathrm{yr}^{-1}$.

The late stages of stellar evolution are governed by the Chandrasekhar mass $(\hbar c / G)^{3 / 2} m_{\mathrm{n}}^{-2}$ mainly determined by the balance between the Fermi pressure of a degenerate electron gas and gravity.

Simple analytical models of the light curves of Type Ia supernovae predict that the peak of luminosity is proportional to the mass of nickel synthesized. In a good approximation, it is a fixed fraction of the Chandrasekhar mass. In models allowing for a varying $G$, this would induce a modification of the luminosity distance-redshift relation [227, 232, 435]. However, it was shown that this effect is small. Note that it will be degenerate with the cosmological parameters. In particular, the Hubble diagram is sensitive to the whole history of $G(t)$ between the highest redshift observed and today so that one needs to rely on a better defined model, such as, e.g., scalar-tensor theory [435] (the effect of the Fermi constant was also considered in [194]).

In the case of Type II supernovae, the Chandrasekhar mass also governs the late evolutionary stages of massive stars, including the formation of neutron stars. Assuming that the mean neutron star mass is given by the Chandrasekhar mass, one expects that $\dot{G} / G=-2 \dot{M}_{\mathrm{NS}} / 3 M_{\mathrm{NS}}$. Thorsett [492] used the observations of five neutron star binaries for which five Keplerian parameters can be determined (the binary period $P_{b}$, the projection of the orbital semi-major axis $a_{1} \sin i$, the eccentricity $e$, the time and longitude of the periastron $T_{0}$ and $\omega$ ) as well as the relativistic 
advance of the angle of the periastron $\dot{\omega}$. Assuming that the neutron star masses vary slowly as $M_{\mathrm{NS}}=M_{\mathrm{NS}}^{(0)}-\dot{M}_{\mathrm{NS}} t_{\mathrm{NS}}$, that their age was determined by the rate at which $P_{b}$ is increasing (so that $t_{N S} \simeq 2 P_{b} / \dot{P}_{b}$ ) and that the mass follows a normal distribution, Thorsett [492] deduced that, at $2 \sigma$,

$$
\dot{G} / G=(-0.6 \pm 4.2) \times 10^{-12} \mathrm{yr}^{-1}
$$

\subsubsection{New developments}

It has recently been proposed that the variation of $G$ inducing a modification of the binary's binding energy, it should affect the gravitational wave luminosity, hence leading to corrections in the chirping frequency [554]. For instance, it was estimated that a LISA observation of an equalmass inspiral event with total redshifted mass of $10^{5} M_{\odot}$ for three years should be able to measure $\dot{G} / G$ at the time of merger to better than $10^{-11} / \mathrm{yr}$. This method paves the way to constructing constraints in a large band of redshifts as well as in different directions in the sky, which would be an invaluable constraint for many models.

More speculative is the idea [25] that a variation of $G$ can lead a neutron to enter into the region where strange or hybrid stars are the true ground state. This would be associated with gamma-ray bursts that are claimed to be able to reach the level of $10^{-17} / \mathrm{yr}$ on the time variation of $G$.

\subsection{Cosmological constraints}

Cosmological observations are more difficult to use in order to set constraints on the time variation of $G$. In particular, they require to have some ideas about the whole history of $G$ as a function of time but also, as the variation of $G$ reflects an extension of General relativity, it requires to modify all equations describing the evolution (of the universe and of the large scale structure) in a consistent way. We refer to $[504,502,506]$ for a discussion of the use of cosmological data to constrain deviations from general relativity.

\subsubsection{Cosmic microwave background}

A time-dependent gravitational constant will have mainly three effects on the CMB angular power spectrum (see [435] for discussions in the framework of scalar-tensor gravity in which $G$ is considered as a field):

1. The variation of $G$ modifies the Friedmann equation and therefore the age of the Universe (and, hence, the sound horizon). For instance, if $G$ is larger at earlier time, the age of the Universe is smaller at recombination, so that the peak structure is shifted towards higher angular scales.

2. The amplitude of the Silk damping is modified. At small scales, viscosity and heat conduction in the photon-baryon fluid produce a damping of the photon perturbations. The damping scale is determined by the photon diffusion length at recombination, and therefore depends on the size of the horizon at this epoch, and hence, depends on any variation of the Newton constant throughout the history of the Universe.

3. The thickness of the last scattering surface is modified. In the same vein, the duration of recombination is modified by a variation of the Newton constant as the expansion rate is different. It is well known that CMB anisotropies are affected on small scales because the last scattering "surface" has a finite thickness. The net effect is to introduce an extra, roughly exponential, damping term, with the cutoff length being determined by the thickness of the last scattering surface. When translating redshift into time (or length), one has to use the

Living Reviews in Relativity

http: //www. livingreviews.org/lrr-2011-2 
Friedmann equations, which are affected by a variation of the Newton constant. The relevant quantity to consider is the visibility function $g$. In the limit of an infinitely thin last scattering surface, $\tau$ goes from $\infty$ to 0 at recombination epoch. For standard cosmology, it drops from a large value to a much smaller one, and hence, the visibility function still exhibits a peak, but it is much broader.

In full generality, the variation of $G$ on the CMB temperature anisotropies depends on many factors: (1) modification of the background equations and the evolution of the universe, (2) modification of the perturbation equations, (3) whether the scalar field inducing the time variation of $G$ is negligible or not compared to the other matter components, (4) on the time profile of $G$ that has to be determine to be consistent with the other equations of evolution. This explains why it is very difficult to state a definitive constraint. For instance, in the case of scalar-tensor theories (see below), one has two arbitrary functions that dictate the variation of $G$. As can be seen, e.g., from $[435,378]$, the profiles and effects on the CMB can be very different and difficult to compare. Indeed, the effects described above are also degenerate with a variation of the cosmological parameters.

In the case of Brans-Dicke theory, one just has a single constant parameter $\omega_{\mathrm{BD}}$ characterizing the deviation from general relativity and the time variation of $G$. Thus, it is easier to compare the different constraints. Chen and Kamionkowski [94] showed that CMB experiments such as WMAP will be able to constrain these theories for $\omega_{\mathrm{BD}}<100$ if all parameters are to be determined by the same CMB experiment, $\omega_{\mathrm{BD}}<500$ if all parameters are fixed but the CMB normalization and $\omega_{\mathrm{BD}}<800$ if one uses the polarization. For the Planck mission these numbers are respectively, 800, 2500 and 3200. [2] concluded from the analysis of WMAP, ACBAR, VSA and CBI, and galaxy power spectrum data from $2 \mathrm{dF}$, that $\omega_{\mathrm{BD}}>120$, in agreement with the former analysis of [378]. An analysis [549] indictates that The 'WMAP-5yr data' and the 'all CMB data' both favor a slightly non-zero (positive) $\dot{G} / G$ but with the addition of the SDSS poser spectrum data, the best-fit value is back to zero, concluding that $-0.083<\Delta G / G<0.095$ between recombination and today, which corresponds to $-1.75 \times 10^{-12} \mathrm{yr}^{-1}<\dot{G} / G<1.05 \times 10^{-12} \mathrm{yr}^{-1}$.

From a more phenomenological prospect, some works modeled the variation of $G$ with time in a purely ad-hoc way, for instance [89] by assuming a linear evolution with time or a step function.

\subsubsection{BBN}

As explained in detail in Section 3.8.1, changing the value of the gravitational constant affects the freeze-out temperature $T_{\mathrm{f}}$. A larger value of $G$ corresponds to a higher expansion rate. This rate is determined by the combination $G \rho$ and in the standard case the Friedmann equations imply that $G \rho t^{2}$ is constant. The density $\rho$ is determined by the number $N_{*}$ of relativistic particles at the time of nucleosynthesis so that nucleosynthesis allows to put a bound on the number of neutrinos $N_{\nu}$. Equivalently, assuming the number of neutrinos to be three, leads to the conclusion that $G$ has not varied from more than $20 \%$ since nucleosynthesis. But, allowing for a change both in $G$ and $N_{\nu}$ allows for a wider range of variation. Contrary to the fine structure constant the role of $G$ is less involved.

The effect of a varying $G$ can be described, in its most simple but still useful form, by introducing a speed-up factor, $\xi=H / H_{G R}$, that arises from the modification of the value of the gravitational constant during BBN. Other approaches considered the full dynamics of the problem but restricted themselves to the particular class of Jordan-Fierz-Brans-Dicke theory [1, 16, 26, 84, 102, 128, 441, 551] (Casas et al. [84] concluded from the study of helium and deuterium that $\omega_{\mathrm{BD}}>380$ when $N_{\nu}=3$ and $\omega_{\mathrm{BD}}>50$ when $N_{\nu}=2$.), of a massless dilaton with a quadratic coupling $[105,106$, $134,446]$ or to a general massless dilaton [455]. It should be noted that a combined analysis of BBN and CMB data was investigated in [113, 292]. The former considered $G$ constant during BBN while the latter focused on a nonminimally quadratic coupling and a runaway potential. It 
was concluded that from the BBN in conjunction with WMAP determination of $\eta$ set that $\Delta G / G$ has to be smaller than $20 \%$. However, we stress that the dynamics of the field can modify CMB results (see previous Section 4.4.1) so that one needs to be careful while inferring $\Omega_{\mathrm{b}}$ from WMAP unless the scalar-tensor theory has converged close to general relativity at the time of decoupling.

In early studies, Barrow [26] assumed that $G \propto t^{-n}$ and obtained from the helium abundances that $-5.9 \times 10^{-3}<n<7 \times 10^{-3}$, which implies that $|\dot{G} / G|<(2 \pm 9.3) h \times 10^{-12} \mathrm{yr}^{-1}$, assuming a flat universe. This corresponds in terms of the Brans-Dicke parameter to $\omega_{\mathrm{BD}}>25$. Yang et al. [551] included the deuterium and lithium to improve the constraint to $n<5 \times 10^{-3}$, which corresponds to $\omega_{\mathrm{BD}}>50$. It was further improved by Rothman and Matzner [441] to $|n|<3 \times 10^{-3}$ implying $|\dot{G} / G|<1.7 \times 10^{-13} \mathrm{yr}^{-1}$. Accetta et al. [1] studied the dependence of the abundances of $\mathrm{D},{ }^{3} \mathrm{He},{ }^{4} \mathrm{He}$ and ${ }^{7} \mathrm{Li}$ upon the variation of $G$ and concluded that $-0.3<\Delta G / G<0.4$, which roughly corresponds to $|\dot{G} / G|<9 \times 10^{-13} \mathrm{yr}^{-1}$. All these investigations assumed that the other constants are kept fixed and that physics is unchanged. Kolb et al. [295] assumed a correlated variation of $G, \alpha_{\mathrm{EM}}$ and $G_{\mathrm{F}}$ and got a bound on the variation of the radius of the extra dimensions.

Although the uncertainty in the helium-4 abundance has been argued to be significantly larger that what was assumed in the past [401], interesting bounds can still be derived [117]. In particular translating the bound on extra relativistic degress of freedom $\left(-0.6<\delta N_{\nu}<0.82\right)$ to a constraint on the speed-up factor $(0.949<\xi<1.062)$, it was concluded [117], since $\Delta G / G=\xi^{2}-1=$ $7 \delta N_{\nu} / 43$, that

$$
-0.10<\frac{\Delta G}{G}<0.13
$$

The relation between the speed-up factor, or an extra number of relativistic degrees of freedom, with a variation of $G$ is only approximate since it assumes that the variation of $G$ affects only the Friedmann equation by a renormalization of $G$. This is indeed accurate only when the scalar field is slow-rolling. For instance [105], the speed-up factor is given (with the notations of Section 5.1.1) by

$$
\xi=\frac{A\left(\varphi_{*}\right)}{A_{0}} \frac{1+\alpha\left(\varphi_{*}\right) \varphi_{*}^{\prime}}{\sqrt{1-\varphi_{*}^{2 \prime} / 3}} \frac{1}{\sqrt{1+\alpha_{0}^{2}}}
$$

so that

$$
\xi^{2}=\frac{G}{G_{0}} \frac{\left(1+\alpha\left(\varphi_{*}\right) \varphi_{*}^{\prime}\right)^{2}}{\left(1+\alpha^{2}\right)\left(1-\varphi_{*}^{2 \prime} / 3\right)},
$$

so that $\Delta G / G_{0}=\xi^{2}-1$ only if $\alpha \ll 1$ (small deviation from general relativity) and $\varphi_{*}^{\prime} \ll 1$ (slow rolling dilaton). The BBN in scalar-tensor theories was investigated $[105,134]$ in the case of a twoparameter family involving a non-linear scalar field-matter coupling function. They concluded that even in the cases where before BBN the scalar-tensor theory was far from general relativity, BBN enables to set quite tight constraints on the observable deviations from general relativity today. In particular, neglecting the cosmological constant, BBN imposes $\alpha_{0}^{2}<10^{-6.5} \beta^{-1}\left(\Omega_{\text {mat }} h^{2} / 0.15\right)^{-3 / 2}$ when $\beta>0.5$ (with the definitions introduced below Equation (164)). 


\section{Theories With Varying Constants}

As explained in the introduction, Dirac postulated that $G$ varies as the inverse of the cosmic time. Such an hypothesis is indeed not a theory since the evolution of $G$ with time is postulated and not derived from an equation of evolution ${ }^{12}$ consistent with the other field equations, that have to take into account that $G$ is no more a constant (in particular in a Lagrangian formulation one needs to take into account that $G$ is no more constant when varying.

The first implementation of Dirac's phenomenological idea into a field-theory framework (i.e., modifying Einstein's gravity and incorporating non-gravitational forces and matter) was proposed by Jordan [268]. He realized that the constants have to become dynamical fields and proposed the action

$$
S=\int \sqrt{-g} \mathrm{~d}^{4} \mathbf{x} \phi^{\eta}\left[R-\xi\left(\frac{\nabla \phi}{\phi}\right)^{2}-\frac{\phi}{2} F^{2}\right]
$$

$\eta$ and $\xi$ being two parameters. It follows that both $G$ and the fine-structure constant have been promoted to the status of a dynamical field.

Fierz [195] realized that with such a Lagrangian, atomic spectra will be space-time-dependent, and he proposed to fix $\eta$ to the value -1 to prevent such a space-time dependence. This led to the definition of a one-parameter $(\xi)$ class of scalar-tensor theories in which only $G$ is assumed to be a dynamical field. This was then further explored by Brans and Dicke [67] (with the change of notation $\xi \rightarrow \omega)$. In this Jordan-Fierz-Brans-Dicke theory the gravitational constant is replaced by a scalar field, which can vary both in space and time. It follows that, for cosmological solutions, $G \propto t^{-n}$ with $n^{-1}=2+3 \omega_{\mathrm{BD}} / 2$. Thus, Einstein's gravity is recovered when $\omega_{\mathrm{BD}} \rightarrow \infty$. This kind of theory was further generalized to obtain various functional dependencies for $G$ in the formalisation of scalar-tensor theories of gravitation (see, e.g., Damour and Esposito-Farèse [124] or Will [540]).

\subsection{Introducing new fields: generalities}

\subsubsection{The example of scalar-tensor theories}

Let us start to remind how the standard general relativistic framework can be extended to make $G$ dynamical on the example of scalar-tensor theories, in which gravity is mediated not only by a massless spin- 2 graviton but also by a spin-0 scalar field that couples universally to matter fields (this ensures the universality of free fall). In the Jordan frame, the action of the theory takes the form

$$
S=\int \frac{\mathrm{d}^{4} x}{16 \pi G_{*}} \sqrt{-g}\left[F(\varphi) R-g^{\mu \nu} Z(\varphi) \varphi_{, \mu} \varphi_{, \nu}-2 U(\varphi)\right]+S_{\text {matter }}\left[\psi ; g_{\mu \nu}\right]
$$

where $G_{*}$ is the bare gravitational constant. This action involves three arbitrary functions $(F, Z$ and $U$ ) but only two are physical since there is still the possibility to redefine the scalar field. $F$ needs to be positive to ensure that the graviton carries positive energy. $S_{\text {matter }}$ is the action of the matter fields that are coupled minimally to the metric $g_{\mu \nu}$. In the Jordan frame, the matter is universally coupled to the metric so that the length and time as measured by laboratory apparatus are defined in this frame.

\footnotetext{
12 Note that the Dirac hypothesis can also be achieved by assuming that $e$ varies as $t^{1 / 2}$. Indeed this reflects a choice of units, either atomic or Planck units. However, there is a difference: assuming that only $G$ varies violates the strong equivalence principle while assuming a varying $e$ results in a theory violating the weak equivalence principle. It does not mean that we are detecting the variation of a dimensionful constant but simply that either $e^{2} / \hbar c$ or $G m_{\mathrm{e}}^{2} / \hbar c$ is varying. This shows that many implementations of this idea are a priori possible.
} 
The variation of this action gives the following field equations

$$
\begin{aligned}
F(\varphi)\left(R_{\mu \nu}-\frac{1}{2} g_{\mu \nu} R\right)= & 8 \pi G_{*} T_{\mu \nu}+Z(\varphi)\left[\partial_{\mu} \varphi \partial_{\nu} \varphi-\frac{1}{2} g_{\mu \nu}\left(\partial_{\alpha} \varphi\right)^{2}\right] \\
& +\nabla_{\mu} \partial_{\nu} F(\varphi)-g_{\mu \nu} \square F(\varphi)-g_{\mu \nu} U(\varphi), \\
2 Z(\varphi) \square \varphi= & -\frac{d F}{d \varphi} R-\frac{d Z}{d \varphi}\left(\partial_{\alpha} \varphi\right)^{2}+2 \frac{d U}{d \varphi}, \\
\nabla_{\mu} T_{\nu}^{\mu}= & 0,
\end{aligned}
$$

where $T \equiv T_{\mu}^{\mu}$ is the trace of the matter energy-momentum tensor $T^{\mu \nu} \equiv(2 / \sqrt{-g}) \times \delta S_{m} / \delta g_{\mu \nu}$. As expected [183], we have an equation, which reduces to the standard Einstein equation when $\varphi$ is constant and a new equation to describe the dynamics of the new degree of freedom while the conservation equation of the matter fields is unchanged, as expected from the weak equivalence principle.

It is useful to define an Einstein frame action through a conformal transformation of the metric $g_{\mu \nu}^{*}=F(\varphi) g_{\mu \nu}$. In the following all quantities labeled by a star $\left(^{*}\right)$ will refer to Einstein frame. Defining the field $\varphi_{*}$ and the two functions $A\left(\varphi_{*}\right)$ and $V\left(\varphi_{*}\right)$ (see, e.g., [191]) by

$$
\left(\frac{\mathrm{d} \varphi_{*}}{\mathrm{~d} \varphi}\right)^{2}=\frac{3}{4}\left(\frac{\mathrm{d} \ln F(\varphi)}{\mathrm{d} \varphi}\right)^{2}+\frac{1}{2 F(\varphi)}, \quad A\left(\varphi_{*}\right)=F^{-1 / 2}(\varphi), \quad 2 V\left(\varphi_{*}\right)=U(\varphi) F^{-2}(\varphi),
$$

the action (157) reads as

$$
S=\frac{1}{16 \pi G_{*}} \int \mathrm{d}^{4} x \sqrt{-g_{*}}\left[R_{*}-2 g_{*}^{\mu \nu} \partial_{\mu} \varphi_{*} \partial_{\nu} \varphi_{*}-4 V\right]+S_{\text {matter }}\left[A^{2} g_{\mu \nu}^{*} ; \psi\right] .
$$

The kinetic terms have been diagonalized so that the spin-2 and spin-0 degrees of freedom of the theory are perturbations of $g_{\mu \nu}^{*}$ and $\varphi_{*}$ respectively. In this frame the field equations are given by

$$
\begin{aligned}
R_{\mu \nu}^{*}-\frac{1}{2} R^{*} g_{\mu \nu}^{*} & =8 \pi G_{*} T_{\mu \nu}^{*}+2 \partial_{\mu} \varphi_{*} \partial_{\nu} \varphi_{*}-g_{\mu \nu}^{*}\left(g_{*}^{\alpha \beta} \partial_{\alpha} \varphi_{*} \partial_{\beta} \varphi_{*}\right)-2 V(\varphi) g_{\mu \nu}^{*}, \\
\square_{*} \varphi_{*} & =-4 \pi G_{*} \alpha\left(\varphi_{*}\right) T_{*}+d V(\varphi) / d \varphi_{*}, \\
\nabla_{\mu}^{*} T_{* \nu}^{\mu} & =\alpha\left(\varphi_{*}\right) T_{*} \partial_{\nu} \varphi_{*},
\end{aligned}
$$

with $\alpha \equiv \mathrm{d} \ln A / \mathrm{d} \varphi_{*}$ and $\beta \equiv \mathrm{d} \alpha / \mathrm{d} \varphi_{*}$. In this version, the Einstein equations are not modified, but since the theory can now be seen as the theory in which all the mass are varying in the same way, there is a source term to the conservation equation. This shows that the same theory can be interpreted as a varying $G$ theory or a universally varying mass theory, but remember that whatever its form the important parameter is the dimensionless quantity $G m^{2} / \hbar c$.

The action (157) defines an effective gravitational constant $G_{\mathrm{eff}}=G_{*} / F=G_{*} A^{2}$. This constant does not correspond to the gravitational constant effectively measured in a Cavendish experiment. The Newton constant measured in this experiment is

$$
G_{\mathrm{cav}}=G_{*} A_{0}^{2}\left(1+\alpha_{0}^{2}\right)=\frac{G_{*}}{F}\left(1+\frac{F_{\phi}^{2}}{2 F+3 F_{\phi}^{2}}\right)
$$

where the first term, $G_{*} A_{0}^{2}$ corresponds to the exchange of a graviton while the second term $G_{*} A_{0}^{2} \alpha_{0}^{2}$ is related to the long range scalar force, a subscript 0 referring to the quantity evaluated today. The gravitational constant depends on the scalar field and is thus dynamical.

This illustrates the main features that will appear in any such models: (i) new dynamical fields appear (here a scalar field), (ii) some constant will depend on the value of this scalar field (here $G$ 
is a function of the scalar field). It follows that the Einstein equations will be modified and that there will exist a new equation dictating the propagation of the new degree of freedom.

In this particular example, the coupling of the scalar field is universal so that no violation of the universality of free fall is expected. The deviation from general relativity can be quantified in terms of the post-Newtonian parameters, which can be expressed in terms of the values of $\alpha$ and $\beta$ today as

$$
\gamma^{\mathrm{PPN}}-1=-\frac{2 \alpha_{0}^{2}}{1+\alpha_{0}^{2}}, \quad \beta^{\mathrm{PPN}}-1=\frac{1}{2} \frac{\beta_{0} \alpha_{0}^{2}}{\left(1+\alpha_{0}^{2}\right)^{2}} .
$$

These expressions are valid only if the field is light on the solar system scales. If this is not the case then these conclusions may be changed [287]. The solar system constraints imply $\alpha_{0}$ to be very small, typically $\alpha_{0}^{2}<10^{-5}$ while $\beta_{0}$ can still be large. Binary pulsar observations $[125,189]$ impose that $\beta_{0}>-4.5$. The time variation of $G$ is then related to $\alpha_{0}, \beta_{0}$ and the time variation of the scalar field today

$$
\frac{\dot{G}_{\mathrm{cav}}}{G_{\mathrm{cav}}}=2 \alpha_{0}\left(1+\frac{\beta_{0}}{1+\alpha_{0}^{2}}\right) \dot{\varphi}_{* 0} .
$$

This example shows that the variation of the constant and the deviation from general relativity quantified in terms of the PPN parameters are of the same magnitude, because they are all driven by the same new scalar field.

The example of scalar-tensor theories is also very illustrative to show how deviation from general relativity can be fairly large in the early universe while still being compatible with solar system constraints. It relies on the attraction mechanism toward general relativity [130, 131].

Consider the simplest model of a massless dilaton $\left(V\left(\varphi_{*}\right)=0\right)$ with quadratic coupling $(\ln A=$ $\left.a=\frac{1}{2} \beta \varphi_{*}^{2}\right)$. Note that the linear case correspond to a Brans-Dicke theory with a fixed deviation from general relativity. It follows that $\alpha_{0}=\beta \varphi_{0 *}$ and $\beta_{0}=\beta$. As long as $V=0$, the Klein-Gordon equation can be rewritten in terms of the variable $p=\ln a$ as

$$
\frac{2}{3-\varphi_{*}^{\prime 2}} \varphi_{*}^{\prime \prime}+(1-w) \varphi_{*}^{\prime}=-\alpha\left(\varphi_{*}\right)(1-3 w) .
$$

As emphasized in [130], this is the equation of motion of a point particle with a velocity dependent inertial mass, $m\left(\varphi_{*}\right)=2 /\left(3-\varphi_{*}^{\prime 2}\right)$ evolving in a potential $\alpha\left(\varphi_{*}\right)(1-3 w)$ and subject to a damping force, $-(1-w) \varphi_{*}^{\prime}$. During the cosmological evolution the field is driven toward the minimum of the coupling function. If $\beta>0$, it drives $\varphi_{*}$ toward 0 , that is $\alpha \rightarrow 0$, so that the scalar-tensor theory becomes closer and closer to general relativity. When $\beta<0$, the theory is driven way from general relativity and is likely to be incompatible with local tests unless $\varphi_{*}$ was initially arbitrarily close from 0.

It follows that the deviation from general relativity remains constant during the radiation era (up to threshold effects in the early universe [108, 134] and quantum effects [85]) and the theory is then attracted toward general relativity during the matter era. Note that it implies that postulating a linear or inverse variation of $G$ with cosmic time is actually not realistic in this class of models. Since the theory is fully defined, one can easily compute various cosmological observables (late time dynamics [348], CMB anisotropy [435], weak lensing [449], BBN [105, 106, 134]) in a consistent way and confront them with data.

\subsubsection{Making other constants dynamical}

Given this example, it seems a priori simple to cook up a theory that will describe a varying fine-structure constant by coupling a scalar field to the electromagnetic Faraday tensor as

$$
S=\int\left[\frac{R}{16 \pi G}-2\left(\partial_{\mu} \phi\right)^{2}-\frac{1}{4} B(\phi) F_{\mu \nu}^{2}\right] \sqrt{-g} \mathrm{~d}^{4} x
$$


so that the fine-structure will evolve according to $\alpha=B^{-1}$. However, such an simple implementation may have dramatic implications. In particular, the contribution of the electromagnetic binding energy to the mass of any nucleus can be estimated by the Bethe-Weizäcker formula so that

$$
m_{(A, Z)}(\phi) \supset 98.25 \alpha(\phi) \frac{Z(Z-1)}{A^{1 / 3}} \mathrm{MeV} .
$$

This implies that the sensitivity of the mass to a variation of the scalar field is expected to be of the order of

$$
f_{(A, Z)}=\partial_{\phi} m_{(A, Z)}(\phi) \sim 10^{-2} \frac{Z(Z-1)}{A^{4 / 3}} \alpha^{\prime}(\phi) .
$$

It follows that the level of the violation of the universality of free fall is expected to be of the level of $\eta_{12} \sim 10^{-9} X\left(A_{1}, Z_{1} ; A_{2}, Z_{2}\right)\left(\partial_{\phi} \ln B\right)_{0}^{2}$. Since the factor $X\left(A_{1}, Z_{1} ; A_{2}, Z_{2}\right)$ typically ranges as $\mathcal{O}(0.1-10)$, we deduce that $\left(\partial_{\phi} \ln B\right)_{0}$ has to be very small for the solar system constraints to be satisfied. It follows that today the scalar field has to be very close to the minimum of the coupling function $\ln B$. This led to the idea of the least coupling mechanism [135, 136] discussed in Section 5.4.1. This is indeed very simplistic because this example only takes into account the effect of the electromagnetic binding energy (see Section 6.3).

Let us also note that such a simple coupling cannot be eliminated by a conformal rescaling $g_{\mu \nu}=A^{2}(\phi) g_{\mu \nu}^{*}$ since

$$
\int B(\phi) g^{\mu \rho} g^{\mu \nu} F_{\nu \sigma} F_{\rho \sigma} \sqrt{-g} \mathrm{~d}^{4} x \longrightarrow \int B(\phi) A^{D-4}(\phi) g_{*}^{\mu \rho} g_{*}^{\mu \nu} F_{\nu \sigma} F_{\rho \sigma} \sqrt{-g_{*}} \mathrm{~d}^{4} x
$$

so that the action is invariant in $D=4$ dimensions.

This example shows that we cannot couple a field blindly to, e.g., the Faraday tensor to make the fine-structure constant dynamics and that some mechanism for reconciling this variation with local constraints, and in particular the university of free fall, will be needed.

\subsection{High-energy theories and varying constants}

\subsubsection{Kaluza-Klein}

Such coupling terms naturally appear when compactifying a higher-dimensional theory. As an example, let us recall the compactification of a 5-dimensional Einstein-Hilbert action ([409], chapter 13)

$$
S=\frac{1}{12 \pi^{2} G_{5}} \int \bar{R} \sqrt{-\bar{g}} \mathrm{~d}^{5} x .
$$

Decomposing the 5-dimensional metric $\bar{g}_{A B}$ as

$$
\bar{g}_{A B}=\left(\begin{array}{cc}
g_{\mu \nu}+\frac{A_{\mu} A_{\nu}}{M^{2}} \phi^{2} & \frac{A_{\mu}}{M} \phi^{2} \\
\frac{A_{\nu}}{M} \phi^{2} & \phi^{2}
\end{array}\right),
$$

where $M$ is a mass scale, we obtain

$$
S=\frac{1}{16 \pi G_{*}} \int\left(R-\frac{\phi^{2}}{4 M^{2}} F^{2}\right) \phi \sqrt{-g} \mathrm{~d}^{4} x
$$

where the 4-dimensional gravitational constant is $G_{*}=3 \pi G_{5} / 4 \int \mathrm{d} y$. The scalar field couples explicitly to the kinetic term of the vector field and cannot be eliminated by a redefinition of the metric: again, this is the well-known conformal invariance of electromagnetism in four dimensions. Such a term induces a variation of the fine-structure constant as well as a violation of the universality of free-fall. Such dependencies of the masses and couplings are generic for higher-dimensional 
theories and in particular string theory. It is actually one of the definitive predictions for string theory that there exists a dilaton, that couples directly to matter [484] and whose vacuum expectation value determines the string coupling constants [546].

In the models by Kaluza [269] and Klein [291] the 5-dimensional spacetime was compactified assuming that one spatial extra-dimension $S^{1}$, of radius $R_{\mathrm{KK}}$. It follows that any field $\chi\left(x^{\mu}, y\right)$ can be Fourier transformed along the compact dimension (with coordinate $y$ ), so that, from a 4-dimensional point of view, it gives rise to a tower of of fields $\chi^{(n)}\left(x^{\mu}\right)$ of mas $m_{\mathrm{n}}=n R_{K K}$. At energies small compared to $R_{K K}^{-1}$ only the $y$-independent part of the field remains and the physics looks 4-dimensional.

Assuming that the action (171) corresponds to the Jordan frame action, as the coupling $\phi R$ may suggest, it follows that the gravitational constant and the Yang-Mills coupling associated with the vector field $A^{\mu}$ must scale as

$$
G \propto \phi^{-1}, \quad g_{Y M}^{-2} \propto \phi^{2} / G \propto \phi^{3} .
$$

Note that the scaling of $G$ with $\phi$ (or time) is not the one of the gravitational constant that would be measured in a Cavendish experiment since Eq. (165) tells us that $G_{\mathrm{cav}} \propto G_{*} \phi^{-1}\left(1+\frac{1}{2 \phi+3}\right)$.

This can be generalized to the case of $D$ extra-dimensions [114] to

$$
G \propto \phi^{-D}, \quad \alpha_{i}\left(m_{\mathrm{KK}}\right)=K_{i}(D) G \phi^{-2}
$$

where the constants $K_{i}$ depends only on the dimension and topology of the compact space [525] so that the only fundamental constant of the theory is the mass scale $M_{4+D}$ entering the $4+D$ dimensional theory. A theory on $\mathcal{M}_{4} \times \mathcal{M}_{D}$ where $\mathcal{M}_{D}$ is a $D$-dimensional compact space generates a low-energy quantum field theory of the Yang-Mills type related to the isometries of $\mathcal{M}_{D}$ [for instance [545] showed that for $D=7$, it can accommodate the Yang-Mills group $S U(3) \times S U(2) \times$ $U(1)]$. The two main problems of these theories are that one cannot construct chiral fermions in four dimensions by compactification on a smooth manifold with such a procedure and that gauge theories in five dimensions or more are not renormalizable.

In such a framework the variation of the gauge couplings and of the gravitational constant arises from the variation of the size of the extra dimensions so that one can derives stronger constraints that by assuming independent variation, but at the expense of being more model-dependent. Let us mention the works by Marciano [345] and Wu and Wang [550] in which the structure constants at lower energy are obtained by the renormalization group, and the work by Veneziano [515] for a toy model in $D \geq 4$ dimensions, endowed with an invariant UV cut-off $\Lambda$, and containing a large number $N$ of non-self-interacting matter species.

Ref. [295] used the variation (173) to constrain the time variation of the radius of the extra dimensions during primordial nucleosynthesis to conclude that $\left|\Delta R_{\mathrm{KK}} / R_{\mathrm{KK}}\right|<1 \%$. [28] took the effects of the variation of $\alpha_{\mathrm{S}} \propto R_{\mathrm{KK}}^{-2}$ and deduced from the helium- 4 abundance that $\left|\Delta R_{\mathrm{KK}} / R_{\mathrm{KK}}\right|<$ $0.7 \%$ and $\left|\Delta R_{\mathrm{KK}} / R_{\mathrm{KK}}\right|<1.1 \%$ respectively for $D=2$ and $D=7$ Kaluza-Klein theory and that $\left|\Delta R_{\mathrm{KK}} / R_{\mathrm{KK}}\right|<3.4 \times 10^{-10}$ from the Oklo data. An analysis of most cosmological data (BBN, CMB, quasar etc..) assuming that the extra dimension scales as $R_{0}\left(1+\Delta t^{-3 / 4}\right)$ and $R_{0}[1+\Delta]\left(1-\cos \omega\left(t-t_{0}\right)\right)$ concluded that $\Delta$ has to be smaller than $10^{-16}$ and $10^{-8}$ respectively [311], while [330] assumes that gauge fields and matter fields can propagate in the bulk, that is in the extra dimensions. Ref. [336] evaluated the effect of such a couple variation of $G$ and the structures constants on distant supernova data, concluding that a variation similar to the one reported in [524] would make the distant supernovae brighter.

\subsubsection{String theory}

There exist five anomaly-free, supersymmetric perturbative string theories respectively known as type I, type IIA, type IIB, $\mathrm{SO}(32)$ heterotic and $E_{8} \times E_{8}$ heterotic theories (see, e.g., [420]). 
One of the definitive predictions of these theories is the existence of a scalar field, the dilaton, that couples directly to matter [484] and whose vacuum expectation value determines the string coupling constant [546]. There are two other excitations that are common to all perturbative string theories, a rank two symmetric tensor (the graviton) $g_{\mu \nu}$ and a rank two antisymmetric tensor $B_{\mu \nu}$. The field content then differs from one theory to another. It follows that the 4-dimensional couplings are determined in terms of a string scale and various dynamical fields (dilaton, volume of compact space, ... ). When the dilaton is massless, we expect three effects: (i) a scalar admixture of a scalar component inducing deviations from general relativity in gravitational effects, (ii) a variation of the couplings and (iii) a violation of the weak equivalence principle. Our purpose is to show how the 4-dimensional couplings are related to the string mass scale, to the dilaton and the structure of the extra dimensions mainly on the example of heterotic theories.

To be more specific, let us consider an example. The two heterotic theories originate from the fact that left- and right-moving modes of a closed string are independent. This reduces the number of supersymmetry to $N=1$ and the quantization of the left-moving modes imposes that the gauge group is either $S O(32)$ or $E_{8} \times E_{8}$ depending on the fermionic boundary conditions. The effective tree-level action is (see, e.g., Ref. [237])

$$
S_{H}=\int \mathrm{d}^{10} \mathbf{x} \sqrt{-g_{10}} \mathrm{e}^{-2 \Phi}\left[M_{H}^{8}\left\{R_{10}+4 \square \Phi-4(\nabla \Phi)^{2}\right\}-\frac{M_{H}^{6}}{4} F_{A B} F^{A B}+\ldots\right] .
$$

When compactified on a 6-dimensional Calabi-Yau space, the effective 4-dimensional action takes the form

$$
S_{H}=\int \mathrm{d}^{4} \mathbf{x} \sqrt{-g_{4}} \phi\left[M_{H}^{8}\left\{R_{4}+\left(\frac{\nabla \phi}{\phi}\right)^{2}-\frac{1}{6}\left(\frac{\nabla V_{6}}{V_{6}}\right)^{2}\right\}-\frac{M_{H}^{6}}{4} F^{2}\right]+\ldots
$$

where $\phi \equiv V_{6} \mathrm{e}^{-2 \Phi}$ couples identically to the Einstein and Yang-Mills terms. It follows that

$$
M_{4}^{2}=M_{H}^{8} \phi, \quad g_{\mathrm{YM}}^{-2}=M_{H}^{6} \phi
$$

at tree-level. Note that to reach this conclusion, one has to assume that the matter fields (in the 'dots' of Equation (175) are minimally coupled to $g_{4}$; see, e.g., [340]).

The strongly coupled $S O(32)$ heterotic string theory is equivalent to the weakly coupled type I string theory. Type I superstring admits open strings, the boundary conditions of which divide the number of supersymmetries by two. It follows that the tree-level effective bosonic action is $N=1$, $D=10$ supergravity, which takes the form, in the string frame,

$$
S_{I}=\int \mathrm{d}^{10} \mathrm{x} \sqrt{-g_{10}} M_{I}^{6} \mathrm{e}^{-\Phi}\left[\mathrm{e}^{-\Phi} M_{I}^{2} R_{10}-\frac{F^{2}}{4}+\ldots\right]
$$

where the dots contains terms describing the dynamics of the dilaton, fermions and other form fields. At variance with (174), the field $\Phi$ couples differently to the gravitational and Yang-Mills terms because the graviton and Yang-Mills fields are respectively excitation of close and open strings. It follows that $M_{I}$ can be lowered even to the weak scale by simply having $\exp \Phi$ small enough. Type I theories require $D 9$-branes for consistency. When $V_{6}$ is small, one can use T-duality (to render $V_{6}$ large, which allows to use a quantum field theory approach) and turn the D9-brane into a $D 3$-brane so that

$$
S_{I}=\int \mathrm{d}^{10} \mathrm{x} \sqrt{-g_{10}} \mathrm{e}^{-2 \Phi} M_{I}^{8} R_{10}-\int \mathrm{d}^{4} \mathrm{x} \sqrt{-g_{4}} \mathrm{e}^{-\Phi} \frac{1}{4} F^{2}+\ldots
$$

where the second term describes the Yang-Mills fields localized on the D3-brane. It follows that

$$
M_{4}^{2}=\mathrm{e}^{-2 \Phi} V_{6} M_{I}^{8}, \quad g_{\mathrm{YM}}^{-2}=\mathrm{e}^{-\Phi}
$$


at tree-level. If one compactifies the $D 9$-brane on a 6 -dimensional orbifold instead of a 6 -torus, and if the brane is localized at an orbifold fixed point, then gauge fields couple to fields $M_{i}$ living only at these orbifold fixed points with a (calculable) tree-level coupling $c_{i}$ so that

$$
M_{4}^{2}=\mathrm{e}^{-2 \Phi} V_{6} M_{I}^{8}, \quad g_{\mathrm{YM}}^{-2}=\mathrm{e}^{-\Phi}+c_{i} M_{i} .
$$

The coupling to the field $c_{i}$ is a priori non universal. At strong coupling, the 10-dimensional $E_{8} \times E_{8}$ heterotic theory becomes M-theory on $R^{10} \times S^{1} / Z_{2}$ [255]. The gravitational field propagates in the 11-dimensional space while the gauge fields are localized on two 10-dimensional branes.

At one-loop, one can derive the couplings by including Kaluza-Klein excitations to get [163]

$$
g_{\mathrm{YM}}^{-2}=M_{H}^{6} \phi-\frac{b_{a}}{2}\left(R M_{H}\right)^{2}+\ldots
$$

when the volume is large compared to the mass scale and in that case the coupling is no more universal. Otherwise, one would get a more complicated function. Obviously, the 4-dimensional effective gravitational and Yang-Mills couplings depend on the considered superstring theory, on the compactification scheme but in any case they depend on the dilaton.

As an example, [340] considered the $(N=1, D=10)$-supergravity model derived from the heterotic superstring theory in the low energy limit and assumed that the 10-dimensional spacetime is compactified on a 6 -torus of radius $R\left(x^{\mu}\right)$ so that the effective 4-dimensional theory described by (175) is of the Brans-Dicke type with $\omega=-1$. Assuming that $\phi$ has a mass $\mu$, and couples to the matter fluid in the universe as $S_{\text {matter }}=\int \mathrm{d}^{10} \mathbf{x} \sqrt{-g_{10}} \exp (-2 \Phi) \mathcal{L}_{\text {matter }}\left(g_{10}\right)$, the reduced 4-dimensional matter action is

$$
S_{\text {matter }}=\int \mathrm{d}^{4} \mathbf{x} \sqrt{-g} \phi \mathcal{L}_{\text {matter }}(g)
$$

The cosmological evolution of $\phi$ and $R$ can then be computed to deduce that $\alpha_{\mathrm{EM}} / \alpha_{\mathrm{EM}} \simeq 10^{10}$ $(\mu / 1 \mathrm{eV})^{-2} \mathrm{yr}^{-1}$. considered the same model but assumed that supersymmetry is broken by nonperturbative effects such as gaugino condensation. In this model, and contrary to [340], $\phi$ is stabilized and the variation of the constants arises mainly from the variation of $R$ in a runaway potential.

Ref. [290] considers a probe D3-brane probe in the context of AdS/CFT correspondence at finite temperature and provides the predictions for the running electric and magnetic effective couplings, beyond perturbation theory. It allows to construct a varying speed of light model.

To conclude, superstring theories offer a natural theoretical framework to discuss the value of the fundamental constants since they become expectation values of some fields. This is a first step towards their understanding but yet, no complete and satisfactory mechanism for the stabilization of the extra dimensions and dilaton is known.

It has paved the way for various models that we detail in Section 5.4.

\subsection{Relations between constants}

There are different possibilities to relate the variations of different constants. First, in quantum field theory, we have to take into account the running of coupling constants with energy and the possibilities of grand unification to relate them. It will also give a link between the QCD scale, the coupling constants and the mass of the fundamental particles (i.e., the Yukawa couplings and the Higgs vev). Second, one can compute the binding energies and the masses of the proton, neutron and different nuclei in terms of the gauge couplings and the quark masses. This step involves QCD and nuclear physics. Third, one can relate the gyromagnetic factor in terms of the quark masses. This is particularly important to interpret the constraints from the atomic clocks and the QSO spectra. This allows one to set stronger constraints on the varying parameters at the expense of a model-dependence. 


\subsubsection{Implication of gauge coupling unification}

The first theoretical implication of high-energy physics arises from the unification of the nongravitational interactions. In these unification schemes, the three standard model coupling constants derive from one unified coupling constant.

In quantum field, the calculation of scattering processes include higher order corrections of the coupling constants related to loop corrections that introduce some integrals over internal 4momenta. Depending on the theory, these integrals may be either finite or diverging as the logarithm or power law of a UV cut-off. In a class of theories, called renormalizable, among which the standard model of particle physics, the physical quantities calculated at any order do not depend on the choice of the cut-off scale. But the result may depend on $\ln E / m$ where $E$ is the typical energy scale of the process. It follows that the values of the coupling constants of the standard model depend on the energy at which they are measured (or of the process in which they are involved). This running arises from the screening due to the existence of virtual particles, which are polarized by the presence of a charge. The renormalization group allows to compute the dependence of a coupling constants as a function of the energy $E$ as

$$
\frac{\mathrm{d} g_{i}(E)}{\mathrm{d} \ln E}=\beta_{i}(E)
$$

where the beta functions, $\beta_{i}$, depend on the gauge group and on the matter content of the theory and may be expended in powers of $g_{i}$. For the $\mathrm{SU}(2)$ and $\mathrm{U}(1)$ gauge couplings of the standard model, they are given by

$$
\beta_{2}\left(g_{2}\right)=-\frac{g_{2}^{3}}{4 \pi^{2}}\left(\frac{11}{6}-\frac{n_{g}}{3}\right), \quad \beta_{1}\left(g_{1}\right)=+\frac{g_{1}^{3}}{4 \pi^{2}} \frac{5 n_{g}}{9}
$$

where $n_{g}$ is the number of generations for the fermions. We remind that the fine-structure constant is defined in the limit of zero momentum transfer so that cosmological variation of $\alpha_{\mathrm{EM}}$ are independent of the issue of the renormalization group dependence. For the $\mathrm{SU}(3)$ sector, with fundamental Dirac fermion representations,

$$
\beta_{3}\left(g_{3}\right)=-\frac{g_{3}^{3}}{4 \pi^{2}}\left(\frac{11}{4}-\frac{n_{f}}{6}\right)
$$

$n_{f}$ being the number of quark flavors with mass smaller than $E$. The negative sign implies that (1) at large momentum transfer the coupling decreases and loop corrections become less and less significant: QCD is said to be asymptotically free; (2) integrating the renormalization group equation for $\alpha_{3}$ gives

$$
\alpha_{3}(E)=\frac{6 \pi}{\left(33-n_{f}\right) \ln \left(E / \Lambda_{c}\right)}
$$

so that it diverges as the energy scale approaches $\Lambda_{c}$ from above, that we decided to call $\Lambda_{\mathrm{QCD}}$. This scale characterizes all QCD properties and in particular the masses of the hadrons are expected to be proportional to $\Lambda_{\mathrm{QCD}}$ up to corrections of order $m_{\mathrm{q}} / \Lambda_{\mathrm{QCD}}$.

It was noticed quite early that these relations imply that the weaker gauge coupling becomes stronger at high energy, while the strong coupling becomes weaker so that one can thought the three non-gravitational interactions may have a single common coupling strength above a given energy. This is the driving idea of Grand Unified Theories (GUT) in which one introduces a mechanism of symmetry-breaking from a higher symmetry group, such, e.g., as $\mathrm{SO}(10)$ or $\mathrm{SU}(5)$, at high energies. It has two important consequences for our present considerations. First there may exist algebraic relations between the Yukawa couplings of the standard model. Second, the structure constants of the standard model unify at an energy scale $M_{U}$

$$
\alpha_{1}\left(M_{U}\right)=\alpha_{2}\left(M_{U}\right)=\alpha_{3}\left(M_{U}\right) \equiv \alpha_{U}\left(M_{U}\right) .
$$


We note that the electroweak mixing angle, i.e., the can also be time dependent parameter, but only for $E \neq M_{U}$ since at $E=M_{U}$, it is fixed by the symmetry to have the value $\sin ^{2} \theta=3 / 8$, from which we deduce that

$$
\alpha_{\mathrm{EM}}^{-1}\left(M_{Z}\right)=\frac{5}{3} \alpha_{1}^{-1}\left(M_{Z}\right)+\alpha_{2}^{-1}\left(M_{Z}\right)
$$

It follows from the renormalization group relations that

$$
\alpha_{i}^{-1}(E)=\alpha_{i}^{-1}\left(M_{U}\right)-\frac{b_{i}}{2 \pi} \ln \frac{E}{M_{U}}
$$

where the beta-function coefficients are given by $b_{i}=(41 / 10,-19 / 6,7)$ for the standard model (or below the SUSY scale $\left.\Lambda_{\text {SUSY }}\right)$ and by $b_{i}=(33 / 5,1,-3)$ for $N=1$ supersymmetric theory. Given a field decoupling at $m_{\mathrm{th}}$, one has

$$
\alpha_{i}^{-1}\left(E_{-}\right)=\alpha_{i}^{-1}\left(E_{+}\right)-\frac{b_{i}^{(-)}}{2 \pi} \ln \frac{E_{-}}{E_{+}}-\frac{b_{i}^{(\mathrm{th})}}{2 \pi} \ln \frac{m_{\mathrm{th}}}{E_{+}}
$$

where $b_{i}^{(\mathrm{th})}=b^{(+)}-b^{(-)}$with $b^{(+/-)}$the beta-function coefficients respectively above and below the mass threshold, with tree-level matching at $m_{\mathrm{th}}$. In the case of multiple thresholds, one must sum the different contributions. The existence of these thresholds implies that the running of $\alpha_{3}$ is complicated since it depends on the masses of heavy quarks and colored superpartner in the case of supersymmetry. For non-supersymmetric theories, the low-energy expression of the QCD scale is

$$
\Lambda_{\mathrm{QCD}}=E\left(\frac{m_{\mathrm{c}} m_{\mathrm{b}} m_{\mathrm{t}}}{E}\right)^{2 / 27} \exp \left(-\frac{2 \pi}{9 \alpha_{3}(E)}\right)
$$

for $E>m_{\mathrm{t}}$. This implies that the variation of Yukawa couplings, gauge couplings, Higgs vev and $\Lambda_{\mathrm{QCD}} / M_{\mathrm{P}}$ are correlated. A second set of relations arises in models in which the weak scale is determined by dimensional transmutation [184, 185]. In such cases, the Higgs vev is related to the Yukawa constant of the top quark by [77]

$$
v=M_{p} \exp \left(-\frac{8 \pi^{2} c}{h_{\mathrm{t}}^{2}}\right),
$$

where $c$ is a constant of order unity. This would imply that $\delta \ln v=S \delta \ln h$ with $S \sim 160$ [104].

The first consequences of this unification were investigated in Refs. [77, 74, 75, 135, 136, 185, 313] where the variation of the 3 coupling constants was reduced to the one of $\alpha_{U}$ and $M_{U} / M_{\mathrm{P}}$. It was concluded that, setting

$$
R \equiv \delta \ln \Lambda_{\mathrm{QCD}} / \delta \ln \alpha_{\mathrm{EM}}
$$

$R \sim 34$ with a stated accuracy of about $20 \%$ [312, 313] (assuming only $\alpha_{U}$ can vary), $R \sim 40.82$ in the string dilaton model assuming Grand Unification [135, 136] (see Section 5.4.1), $R=38 \pm 6$ [74] and then $R=46[75,76]$, the difference arising from the quark masses and their associated thresholds. However, these results implicitly assume that the electroweak symmetry breaking and supersymmetry breaking mechanisms, as well as the fermion mass generation, are not affected by the variation of the unified coupling. It was also mentioned in [75] that $R$ can reach -235 in unification based on $\mathrm{SU}(5)$ and $\mathrm{SO}(10)$. The large value of $R$ arises from the exponential dependence of $\Lambda_{\mathrm{QCD}}$ on $\alpha_{3}$. In the limit in which the quark masses are set to zero, the proton mass, as well as all other hadronic masses are proportional to $\Lambda_{\mathrm{QCD}}$, i.e., $m_{\mathrm{p}} \propto \Lambda_{\mathrm{QCD}}\left(1+\mathcal{O}\left(m_{\mathrm{q}} / \Lambda_{\mathrm{QCD}}\right)\right)$. [313] further relates the Higgs vev to $\alpha_{\mathrm{EM}}$ by $\mathrm{d} \ln v / \mathrm{d} \ln \alpha_{\mathrm{EM}} \equiv \kappa$ and estimated that $\kappa \sim 70$ so that, assuming that the variation of the Yukawa couplings is negligible, it could be concluded that

$$
\delta \ln \frac{m}{\Lambda_{\mathrm{QCD}}} \sim 35 \delta \ln \alpha_{\mathrm{EM}}
$$


for the quark and electron masses. This would also imply that the variation of $\mu$ and $\alpha_{\mathrm{EM}}$ are correlated, still in a very model-dependent way, typically one can conclude [104] that

$$
\frac{\delta \mu}{\mu}=-0.8 R \frac{\delta \alpha_{\mathrm{EM}}}{\alpha_{\mathrm{EM}}}+0.6(S+1) \frac{\delta h}{h},
$$

with $S \sim 160$. The running of $\alpha_{U}$ can be extrapolated to the Planck mass, $M_{\mathrm{P}}$. Assuming $\alpha_{U}\left(M_{\mathrm{P}}\right)$ fixed and letting $M_{U} / M_{\mathrm{P}}$ vary, it was concluded [153] that $R=2 \pi\left(b_{U}+3\right) /\left[9 \alpha_{\mathrm{EM}}\left(8 b_{U} / 3-12\right)\right]$ where $b_{U}$ is the beta-function coefficient describing the running of $\alpha_{U}$. This shows that a variation of $\alpha_{\mathrm{EM}}$ and $\mu$ can open a windows on GUT theories. A similar analysis [142] assuming that electroweak symmetry breaking was triggered by nonperturbative effects in such a way that $v$ and $\alpha_{U}$ are related, concludes that $\delta \mu / \mu=(13 \pm 7) \delta \alpha_{\mathrm{EM}} / \alpha_{\mathrm{EM}}$ in a theory with soft SUSY breaking and $\delta \mu / \mu=(-4 \pm 5) \delta \alpha_{\mathrm{EM}} / \alpha_{\mathrm{EM}}$ otherwise.

From a phenomenological point of view, [147] making an assumption of proportionality with fixed "unification coefficients" assumes that the variations of the constants at a given redshift $z$ depend on a unique evolution factor $\ell(z)$ and that the variation of all the constants can be derived from those of the unification mass scale (in Planck units), $M_{U}$, the unified gauge coupling $\alpha_{U}$, the Higgs vev, $v$ and in the case of supersymmetric theories the soft supersymmetry breaking mass, $\tilde{m}$. Introducing the coefficients $d_{i}$ by

$$
\Delta \ln \frac{M_{U}}{M_{\mathrm{P}}}=d_{M} \ell, \quad \Delta \ln \alpha_{U}=d_{U} \ell, \quad \Delta \ln \frac{v}{M_{U}}=d_{H} \ell, \quad \Delta \ln \frac{\tilde{m}}{M_{\mathrm{P}}}=d_{S} \ell,
$$

$\left(d_{S}=0\right.$ for non-supersymmetric theories) and assuming that the masses of the standard model fermions all vary with $v$ so that the Yukawa couplings are assumed constant, it was shown that the variations of all constants can be related to $\left(d_{M}, d_{U}, d_{H}, d_{S}\right)$ and $\ell(z)$, using the renormalization group equations (neglecting the effects induced by the variation of $\alpha_{U}$ on the RG running of fermion masses). This decomposition is a good approximation provided that the time variation is slow, which is actually backed up by the existing constraints, and homogeneous in space (so that it may not be applied as such in the case a chameleon mechanism is at work [69]).

This allowed to be defined six classes of scenarios: (1) varying gravitational constant $\left(d_{H}=\right.$ $\left.d_{S}=d_{X}=0\right)$ in which only $M_{U} / M_{\mathrm{P}}$ or equivalently $G \Lambda_{\mathrm{QCD}}^{2}$ is varying; (2) varying unified coupling $\left(d_{U}=1, d_{H}=d_{S}=d_{M}=0\right)$; (3) varying Fermi scale defined by $\left(d_{H}=1, d_{U}=d_{S}=\right.$ $d_{M}=0$ ) in which one has $\mathrm{d} \ln \mu / \mathrm{d} \ln \alpha_{\mathrm{EM}}=-325$; (4) varying Fermi scale and SUSY-breaking scale $\left(d_{S}=d_{H}=1, d_{U}=d_{M}=0\right)$ and for which $\mathrm{d} \ln \mu / \mathrm{d} \ln \alpha_{\mathrm{EM}}=-21.5 ;$ (5) varying unified coupling and Fermi scale $\left(d_{X}=1, d_{H}=\tilde{\gamma} d_{X}, d_{S}=d_{M}=0\right)$ and for which $\mathrm{d} \ln \mu / \mathrm{d} \ln \alpha_{\mathrm{EM}}=$ $(23.2-0.65 \tilde{\gamma}) /(0.865+0.02 \tilde{\gamma}) ;(6)$ varying unified coupling and Fermi scale with SUSY $\left(d_{X}=\right.$ $\left.1, d_{S} \simeq d_{H}=\tilde{\gamma} d_{X}, d_{M}=0\right)$ and for which $\mathrm{d} \ln \mu / \mathrm{d} \ln \alpha_{\mathrm{EM}}=(14-0.28 \tilde{\gamma}) /(0.52+0.013 \tilde{\gamma})$.

Each scenario can be compared to the existing constraints to get sharper bounds on them [146, 147, 149, 364] and emphasize that the correlated variation between different constants (here $\mu$ and $\left.\alpha_{\mathrm{EM}}\right)$ depends strongly on the theoretical hypothesis that are made.

\subsubsection{Masses and binding energies}

The previous Section 5.3.1 described the unification of the gauge couplings. When we consider "composite" systems such as proton, neutron, nuclei or even planets and stars, we need to compute their mass, which requires to determine their binding energy. As we have already seen, the electromagnetic binding energy induces a direct dependence on $\alpha_{\mathrm{EM}}$ and can be evaluated using, e.g., the Bethe-Weizäcker formula (61). The dependence of the masses on the quark masses, via nuclear interactions, and the determination of the nuclear binding energy are especially difficult to estimate.

Living Reviews in Relativity

http: //www . livingreviews . org/lrr-2011-2 
In the chiral limit of QCD in which all quark masses are negligible compared to $\Lambda_{\mathrm{QCD}}$ all dimensionful quantities scale as some power of $\Lambda_{\mathrm{QCD}}$. For instance, concerning the nucleon mass, $m_{\mathrm{N}}=c \Lambda_{\mathrm{QCD}}$ with $c \sim 3.9$ being computed from lattice QCD. This predicts a mass of order $860 \mathrm{MeV}$, smaller than the observed value of $940 \mathrm{MeV}$. The nucleon mass can be computed in chiral perturbation theory and expressed in terms of the pion mass as [316] $m_{\mathrm{N}}=a_{0}+a_{2} m_{\pi}^{2}+$ $a_{4} m_{\pi}^{4}+a_{6} m_{\pi}^{6}+\sigma_{N \pi}+\sigma_{\Delta \pi}+\sigma_{\mathrm{tad}}$ (where all coefficients of this expansion are defined in [316]), which can be used to show [204] that the nucleon mass is scaling as

$$
m_{\mathrm{N}} \propto \Lambda_{\mathrm{QCD}} X_{\mathrm{q}}^{0.037} X_{\mathrm{s}}^{0.011} .
$$

(Note, however, that such a notation is dangerous since it would imply that $m_{\mathrm{N}}$ vanishes in the chiral limit but it is a compact way to give $\delta m_{\mathrm{N}} / \delta X_{\mathrm{q}}$ etc.). It was further extended [208] by using a sigma model to infer that $m_{\mathrm{N}} \propto \Lambda_{\mathrm{QCD}} X_{\mathrm{q}}^{0.045} X_{\mathrm{s}}^{0.19}$. These two examples explicitly show the strong dependence in nuclear modeling.

To go further and determine the sensitivity of the mass of a nucleus to the various constant,

$$
m(A, Z)=Z m_{\mathrm{p}}+(A-Z) m_{\mathrm{n}}+Z m_{\mathrm{e}}+E_{\mathrm{S}}+E_{\mathrm{EM}}
$$

one should determine the strong binding energy [see related discussion below Eq. (17)] in function of the atomic number $Z$ and the mass number $A$.

The case of the deuterium binding energy $B_{D}$ has been discussed in different ways (see Section 3.8.3). Many models have been created. A first route relies on the use of the dependence of $B_{D}$ on the pion mass $[188,38,426,553]$, which can then be related to $m_{\mathrm{u}}, m_{\mathrm{d}}$ and $\Lambda_{\mathrm{QCD}}$. A second avenue is to use a sigma model in the framework of the Walecka model [456] in which the potential for the nuclear forces keeps only the $\sigma, \rho$ and $\omega$ meson exchanges [208]. We also emphasize that the deuterium is only produced during $\mathrm{BBN}$, as it is too weakly bound to survive in the regions of stars where nuclear processes take place. The fact that we do observe deuterium today sets a non-trivial constraint on the constants by imposing that the deuterium remains stable from BBN time to today. Since it is weakly bound, it is also more sensitive to a variation of the nuclear force compared to the electromagnetic force. This was used in [145] to constrain the variation of the nuclear strength in a sigma-model.

For larger nuclei, the situation is more complicated since there is no simple modeling. For large mass number $A$, the strong binding energy can be approximated by the liquid drop model

$$
\frac{E_{\mathrm{S}}}{A}=a_{V}-\frac{a_{S}}{A^{1 / 3}}-a_{A} \frac{(A-2 Z)^{2}}{A^{2}}+a_{P} \frac{(-1)^{A}+(-1)^{Z}}{A^{3 / 2}}
$$

$\operatorname{with}\left(a_{V}, a_{S}, a_{A}, a_{P}\right)=(15.7,17.8,23.7,11.2) \mathrm{MeV}$ [439]. It has also been suggested [129] that the nuclear binding energy can be expressed as

$$
E_{\mathrm{S}} \simeq A a_{3}+A^{2 / 3} b_{3} \quad \text { with } \quad a_{3}=a_{3}^{\text {chiral limit }}+m_{\pi}^{2} \frac{\partial a_{3}}{\partial m_{\pi}^{2}} .
$$

In the chiral limit, $a_{3}$ has a non-vanishing limit to which we need to add a contribution scaling like $m_{\pi}^{2} \propto \Lambda_{\mathrm{QCD}} m_{\mathrm{q}}$. [129] also pointed out that the delicate balance between attractive and repulsive nuclear interactions [456] implies that the binding energy of nuclei is expected to depend strongly on the quark masses [159]. Recently, a fitting formula derived from effective field theory and based of the semi-empirical formula derived in [222] was proposed [120] as

$$
\frac{E_{\mathrm{S}}}{A}=-\left(120-\frac{97}{A^{1 / 3}}\right) \eta_{S}+\left(67-\frac{57}{A^{1 / 3}}\right) \eta_{V}+\ldots
$$

where $\eta_{S}$ and $\eta_{V}$ are the strength of respectively the scalar (attractive) and vector (repulsive) nuclear contact interactions normalized to their actual value. These two parameters need to be 
related to the QCD parameters [159]. We also refer to [211] for the study of the dependence of the binding of light $(A \leq 8)$ nuclei on possible variations of hadronic masses, including meson, nucleon, and nucleon-resonance masses.

These expressions allow to compute the sensitivity coefficients that enter in the decomposition of the mass [see Equation (201)]. They also emphasize one of the most difficult issue concerning the investigation about constant related to the intricate structure of QCD and its role in low energy nuclear physics, which is central to determine the masses of nuclei and the binding energies, quantities that are particularly important for BBN, the universality of free fall and stellar physics.

\subsubsection{Gyromagnetic factors}

The constraints arising from the comparison of atomic clocks (see Section 3.1) involve the finestructure constant $\alpha_{\mathrm{EM}}$, the proton-to-electron mass ratio $\mu$ and various gyromagnetic factors. It is important to relate these factors to fundamental constants.

The proton and neutron gyromagnetic factors are respectively given by $g_{\mathrm{p}}=5.586$ and $g_{\mathrm{n}}=$ -3.826 and are expected to depend on $X_{\mathrm{q}}=m_{\mathrm{q}} / \Lambda_{\mathrm{QCD}}$ [197]. In the chiral limit in which $m_{\mathrm{u}}=$ $m_{\mathrm{d}}=0$, the nucleon magnetic moments remain finite so that one could have thought that the finite quark mass effects should be small. However, it is enhanced by $\pi$-meson loop corrections, which are proportional to $m_{\pi} \propto \sqrt{m_{\mathrm{q}} \Lambda_{\mathrm{QCD}}}$. Following [316], this dependence can be described by the approximate formula

$$
g\left(m_{\pi}\right)=\frac{g(0)}{1+a m_{\pi}+b m_{\pi}^{2}} .
$$

The coefficients are given by $a=(1.37,1.85) / \mathrm{GeV}$ and $b=(0.452,0.271) / \mathrm{GeV}^{2}$ respectively for the proton an neutron. This lead [197] to $g_{\mathrm{p}} \propto m_{\pi}^{-0.174} \propto X_{\mathrm{q}}^{-0.087}$ and $g_{\mathrm{n}} \propto m_{\pi}^{-0.213} \propto X_{\mathrm{q}}^{-0.107}$. This was further extended in [204] to take into account the dependence with the strange quark mass $m_{\mathrm{s}}$ to obtain

$$
g_{\mathrm{p}} \propto X_{\mathrm{q}}^{-0.087} X_{\mathrm{s}}^{-0.013}, \quad g_{\mathrm{n}} \propto X_{\mathrm{q}}^{-0.118} X_{\mathrm{s}}^{0.0013} .
$$

All these expressions assumes $\Lambda_{\mathrm{QCD}}$ constant in their derivations.

This allows one to express the results of atomic clocks (see Section 3.1.3) in terms of $\alpha_{\mathrm{EM}}, X_{\mathrm{q}}$, $X_{\mathrm{s}}$ and $X_{\mathrm{e}}$. Similarly, for the constants constrained by QSO observation, we have (see Table 10)

$$
\begin{aligned}
x & \propto \alpha_{\mathrm{EM}}^{2} X_{\mathrm{q}}^{-0.087} X_{\mathrm{s}}^{-0.013}, \\
y & \propto \alpha_{\mathrm{EM}}^{2} X_{\mathrm{q}}^{-0.124} X_{\mathrm{s}}^{-0.024} X_{\mathrm{e}}, \\
\bar{\mu} & \propto X_{\mathrm{q}}^{-0.037} X_{\mathrm{s}}^{-0.011} X_{\mathrm{e}} \\
F & \propto \alpha_{\mathrm{EM}}^{3.14} X_{\mathrm{q}}^{-0.0289} X_{\mathrm{s}}^{0.0043} X_{\mathrm{e}}^{-1.57}, \\
F^{\prime} & \propto \alpha_{\mathrm{EM}}^{2} X_{\mathrm{q}}^{0.037} X_{\mathrm{s}}^{0.011} X_{\mathrm{e}}^{-1}, \\
G & \propto \alpha_{\mathrm{EM}}^{1.85} X_{\mathrm{q}}^{-0.0186} X_{\mathrm{s}}^{0.0073} X_{\mathrm{e}}^{-1.85},
\end{aligned}
$$

once the scaling of the nucleon mass as $m_{\mathrm{N}} \propto \Lambda_{\mathrm{QCD}} X_{\mathrm{q}}^{0.037} X_{\mathrm{s}}^{0.011}$ (see Section 5.3.2). This shows that the seven observable quantities that are constrained by current QSO observations can be reduced to only 4 parameters.

\subsection{Models with varying constants}

The models that can be constructed are numerous and cannot all be reviewed here. Thus, we focus on the string dilaton model in Section 5.4.1 and then discuss the chameleon mechanism in Section 5.4.2 and the Bekenstein framework in Section 5.4.3. 


\subsubsection{String dilaton and Runaway dilaton models}

Damour and Polyakov $[135,136]$ argued that the effective action for the massless modes taking into account the full string loop expansion should be of the form

$$
\begin{aligned}
S= & \int \mathrm{d}^{4} \mathbf{x} \sqrt{-\hat{g}}\left[M_{s}^{2}\left\{B_{g}(\Phi) \hat{R}+4 B_{\Phi}(\Phi)\left[\hat{\square} \Phi-(\hat{\nabla} \Phi)^{2}\right]\right\}-B_{F}(\Phi) \frac{k}{4} \hat{F}^{2}\right. \\
& \left.-B_{\psi}(\Phi) \overline{\hat{\psi}} \hat{D} \hat{\psi}+\ldots\right]
\end{aligned}
$$

in the string frame, $M_{s}$ being the string mass scale. The functions $B_{i}$ are not known but can be expanded (from the genus expansion of string theory) in the limit $\Phi \rightarrow-\infty$ as

$$
B_{i}(\Phi)=\mathrm{e}^{-2 \Phi}+c_{0}^{(i)}+c_{1}^{(i)} \mathrm{e}^{2 \Phi}+c_{2}^{(i)} \mathrm{e}^{4 \Phi}+\ldots
$$

where the first term is the tree level term. It follows that these functions can exhibit a local maximum. After a conformal transformation $\left(g_{\mu \nu}=C B_{g} \hat{g}_{\mu \nu}, \psi=\left(C B_{g}\right)^{-3 / 4} B_{\psi}^{1 / 2} \hat{\psi}\right)$, the action in Einstein frame takes the form

$$
S=\int \frac{\mathrm{d}^{4} \mathbf{x}}{16 \pi G} \sqrt{-g}\left[R-2(\nabla \phi)^{2}-\frac{k}{4} B_{F}(\phi) F^{2}-\bar{\psi} \not \supset \psi+\ldots\right]
$$

where the field $\phi$ is defined as

$$
\phi \equiv \int\left[\frac{3}{4}\left(\frac{B_{g}^{\prime}}{B_{g}}\right)^{2}+2 \frac{B_{\Phi}^{\prime}}{B_{\Phi}}+2 \frac{B_{\Phi}^{\prime}}{B_{g}}\right] \mathrm{d} \Phi .
$$

It follows that the Yang-Mills coupling behaves as $g_{\mathrm{YM}}^{-2}=k B_{F}(\phi)$. This also implies that the QCD mass scale is given by

$$
\Lambda_{\mathrm{QCD}} \sim M_{s}\left(C B_{g}\right)^{-1 / 2} \mathrm{e}^{-8 \pi^{2} k B_{F} / b}
$$

where $b$ depends on the matter content. It follows that the mass of any hadron, proportional to $\Lambda_{\mathrm{QCD}}$ in first approximation, depends on the dilaton, $m_{A}\left(B_{g}, B_{F}, \ldots\right)$.

If, as allowed by the ansatz (195), $m_{A}(\phi)$ has a minimum $\phi_{m}$ then the scalar field will be driven toward this minimum during the cosmological evolution. However, if the various coupling functions have different minima then the minima of $m_{A}(\phi)$ will depend on the particle $A$. To avoid violation of the equivalence principle at an unacceptable level, it is thus necessary to assume that all the minima coincide in $\phi=\phi_{m}$, which can be implemented by setting $B_{i}=B$. This can be realized by assuming that $\phi_{m}$ is a special point in field space, for instance it could be associated to the fixed point of a $Z_{2}$ symmetry of the $T$ - or $S$-duality [129].

Expanding $\ln B$ around its maximum $\phi_{m}$ as $\ln B \propto-\kappa\left(\phi-\phi_{m}\right)^{2} / 2$, Damour and Polyakov [135, 136] constrained the set of parameters $\left(\kappa, \phi_{0}-\phi_{m}\right)$ using the different observational bounds. This toy model allows one to address the unsolved problem of the dilaton stabilization, to study all the experimental bounds together and to relate them in a quantitative manner (e.g., by deriving a link between equivalence-principle violations and time-variation of $\alpha_{\mathrm{EM}}$ ). This model was compared to astrophysical data in [306] to conclude that $|\Delta \phi|<3.4 \kappa 10^{-6}$.

An important feature of this model lies in the fact that at lowest order the masses of all nuclei are proportional to $\Lambda_{\mathrm{QCD}}$ so that at this level of approximation, the coupling is universal and the theory reduces to a scalar-tensor theory and there will be no violation of the universality of free fall. It follows that the deviation from general relativity are characterized by the PPN parameters

$$
\gamma^{\mathrm{PPN}}-1 \simeq-2 f_{A}^{2}=-2 \beta_{s}^{2} \kappa^{2} \Delta \phi_{0}^{2}, \quad \beta^{\mathrm{PPN}}-1 \simeq \frac{1}{2} f_{A}^{2} \frac{\mathrm{d} f_{A}}{\mathrm{~d} \phi}=\frac{1}{2} \beta_{s}^{3} \kappa^{3} \Delta \phi_{0}^{2}
$$


with

$$
f_{A}=\frac{\partial \ln \Lambda_{\mathrm{QCD}}(\phi)}{\partial \phi}=-\left[\ln \frac{M_{s}}{m_{A}}+\frac{1}{2}\right] \frac{\mathrm{d} \ln B}{\mathrm{~d} \phi} \equiv-\beta_{s} \frac{\mathrm{d} \ln B}{\mathrm{~d} \phi}=\beta_{s} \kappa \Delta \phi_{0}
$$

with $\Delta \phi_{0}=\phi_{0}-\phi_{m}$ and $\beta_{s} \sim 40$ [135]. The variation of the gravitational constant is, from Equation (167), simply

$$
\frac{\dot{G}}{G}=2 f_{A} \dot{\phi}_{0}=-2\left[\ln \frac{M_{s}}{m_{A}}+\frac{1}{2}\right] \frac{\mathrm{d} \ln B}{\mathrm{~d} \phi} \dot{\phi}_{0}
$$

The value of $\dot{\phi}_{0}=H_{0} \phi_{0}^{\prime}$ is obtained from the Klein-Gordon equation (168) and is typically given by $\phi_{0}^{\prime}=-Z \beta_{s} \kappa H_{0} \Delta \phi_{0}$ were $Z$ is a number that depends on the equation of state of the fluid dominating the matter content of the universe in the last $e$-fold and the cosmological parameters so that

$$
\left.\frac{\dot{G}}{G}\right|_{0}=2 f_{A} \dot{\phi}_{0}=-2 Z H_{0} \beta_{s}^{2} \kappa^{2} \Delta \phi_{0}^{2}
$$

The factor $Z$ is model-dependent and another way to estimate $\dot{\phi}_{0}$ is to use the Friedmann equations, which imply that $\dot{\phi}_{0}=H_{0} \sqrt{1+q_{0}-\frac{3}{3} \Omega_{\mathrm{m} 0}}$ where $q$ is the deceleration parameter.

When one considers the effect of the quark masses and binding energies, various compositiondependent effects appear. First, the fine-structure constant scales as $\alpha_{\mathrm{EM}} \simeq B^{-1}$ so that

$$
\left.\frac{\dot{\alpha}}{\alpha}\right|_{0}=\kappa \Delta \phi_{0} \dot{\phi}_{0}=-Z H_{0} \beta_{s} \kappa^{2} \Delta \phi_{0}^{2} .
$$

The second effect is, as pointed out earlier, a violation of the universality of free fall. In full generality, we expect that

$$
m_{A}(\phi)=N \Lambda_{\mathrm{QCD}}(\phi)\left[1+\sum_{\mathrm{q}} \epsilon_{A}^{q} \frac{m_{\mathrm{q}}}{\Lambda_{\mathrm{QCD}}}+\epsilon_{A}^{\mathrm{EM}} \alpha_{\mathrm{EM}}\right]
$$

Using an expansion of the form (17), it was concluded that

$$
\eta_{A B}=\kappa^{2}\left(\phi_{0}-\phi_{m}\right)^{2}\left[C_{B} \Delta\left(\frac{B}{M}\right)+C_{D} \Delta\left(\frac{D}{M}\right)+C_{E} \Delta\left(\frac{E}{M}\right)\right]
$$

with $B=N+Z, D=N-Z$ and $E=Z(Z-1) /(N+Z)^{1 / 3}$ and where the value of the parameters $C_{i}$ are model-dependent.

It follows from this model that:

- The PPN parameters, the time variation of $\alpha$ and $G$ today and the violation of the university of free-fall all scale as $\Delta \phi_{0}^{2}$.

- The field is driven toward $\phi_{m}$ during the cosmological evolution, a point at which the scalar field decouples from the matter field. The mechanism is usually called the least coupling principle.

- Once the dynamics for the scalar field is solved, $\Delta \phi_{0}$ can be related to $\Delta \phi_{i}$ at the end of inflation. Interestingly, this quantity can be expressed in terms of amplitude of the density contrast at the end of inflation, that is to the energy scale of inflation.

- The numerical estimations [135] indicate that $\eta_{U, H} \sim-5.4 \times 10^{-5}\left(\gamma^{\mathrm{PPN}}-1\right)$ showing that in such a class of models, the constraint on $\eta \sim 10^{-13}$ implies $1-\gamma^{\mathrm{PPN}} \sim 2 \times 10^{-9}$, which is a better constraint that the one obtained directly. 
This model was extended [133] to the case where the coupling functions have a smooth finite limit for infinite value of the bare string coupling, so that $B_{i}=C_{i}+\mathcal{O}\left(\mathrm{e}^{-\phi}\right)$, folling [229]. The dilaton runs away toward its attractor at infinity during a stage of inflation. The late time dynamics of the scalar field is similar as in quintessence models, so that the model can also explain the late time acceleration of the cosmic expansion. The amplitude of residual dilaton interaction is related to the amplitude of the primordial density fluctuations and it induces a variation of the fundamental constants, provided it couples to dark matter or dark energy. It is concluded that, in this framework, the largest allowed variation of $\alpha_{\mathrm{EM}}$ is of order $2 \times 10^{-6}$, which is reached for a violation of the universality of free fall of order $10^{-12}$ and it was established that

$$
\left.\frac{\alpha_{\mathrm{EM}}}{\alpha_{\mathrm{EM}}}\right|_{0} \sim \pm 10^{-16} \sqrt{1+q_{0}-\frac{3}{2} \Omega_{\mathrm{m} 0}} \sqrt{10^{12} \eta} \mathrm{yr}^{-1}
$$

where the first square-root arises from the computation of $\dot{\phi}_{0}$. The formalism was also used to discuss the time variation of $\alpha_{\mathrm{EM}}$ and $\mu$ [97].

The coupling of the dilaton to the standard model fields was further investigated in [122, 121]. Assuming that the heavy quarks and weak gauge bosons have been integrated out and that the dilaton theory has been matched to the light fields below the scale of the heavy quarks, the coupling of the dilaton has been parameterized by 5 parameters: $d_{e}$ and $d_{g}$ for the couplings to the electromagnetic and gluonic field-strength terms, and $d_{m_{e}}, d_{m_{u}}$ and $d_{m_{d}}$ for the couplings to the fermionic mass terms so that the interaction Lagrangian is reduces to a linear coupling (e.g., $\propto d_{e} \phi F^{2}$ for the coupling to electromagnetism etc.) It follows that $\Delta \alpha_{\mathrm{EM}} / \alpha_{\mathrm{EM}}=d_{e} \kappa \phi$ for the fine structure constant, $\Delta \Lambda_{\mathrm{QCD}} / \Lambda_{\mathrm{QCD}}=d_{d} \kappa \phi$ for the strong sector and $\Delta m_{i} / m_{i}=d_{m_{i}} \kappa \phi$ for the masses of the fermions. These parameters can be constrained by the test of the equivalence principle in the solar system [see Section 6.3].

In these two string-inspired scenarios, the amplitude of the variation of the constants is related to the one of the density fluctuations during inflation and the cosmological evolution.

\subsubsection{The Chameleon mechanism}

A central property of the least coupling principle, that is at the heart of the former models, is that all coupling functions have the same minimum so that the effective potential entering the Klein-Gordon equation for the dilaton has a well-defined minimum.

It was realized [287] that if the dilaton has a coupling $A^{2}(\phi)$ to matter while evolving in a potential $V(\phi)$ the source of the Klein-Gordon equation (168) has a an effective potential

$$
V_{\mathrm{eff}}=V(\phi)+A^{2}(\phi) \rho .
$$

In the case where $V$ is a decreasing function of $\phi$, e.g., a runaway potential, and the coupling is an increasing function, e.g., $A^{2}=\exp \beta \phi / M_{\mathrm{P}}$, the effective potential has a minimum the value of which depends on the matter local density $\rho$ (see also [186]). Thus, the field is expected to be massive on Earth where the density is high and light in space in the solar system. It follows that the experiment on the universality of free fall in space may detect violations of the universality of free fall larger than the bounds derived by laboratory experiments [288, 498]. It follows (1) that the constraints on the time variation of the constants today can be relaxed if such a mechanism is at work and (2) that is the constants depend on the local value of the chameleon field, their value will be environment dependent and will be different on Earth and in space.

The cosmological variation of $\alpha_{\mathrm{EM}}$ in such model was investigated in [70, 71]. Models based on the Lagrangian (209) and exhibiting the chameleon mechanism were investigated in [398].

The possible shift in the value of $\mu$ in the Milky Way (see Section 6.1.3) was related [323, 324, 322 ] to the model of [398] to conclude that such a shift was compatible with this model. 


\subsubsection{Bekenstein and related models}

Bekenstein [39, 40] introduced a theoretical framework in which only the electromagnetic sector was modified by the introduction of a dimensionless scalar field $\epsilon$ so that all electric charges vary in unison $e_{i}=e_{0 i} \epsilon\left(x^{\alpha}\right)$ so that only $\alpha_{\mathrm{EM}}$ is assumed to possibly vary.

To avoid the arbitrariness in the definition of $\epsilon$, which can be rescaled by a constant factor while $e_{0 i}$ is inversely rescales, it was postulated that the dynamics of $\epsilon$ be invariant under global rescaling so that its action should be of the form

$$
S_{\epsilon}=-\frac{\hbar c}{2 l^{2}} \int \frac{g^{\mu \nu} \partial_{\mu} \epsilon \partial_{\nu} \epsilon}{\epsilon^{2}} \sqrt{-g} \mathrm{~d}^{4} x
$$

$l$ being a constant length scale. Then, $\epsilon$ is assumed to enter all electromagnetic interaction via $e_{i} A_{\mu} \rightarrow e_{0 i} \epsilon A_{\mu}$ where $A_{\mu}$ is the usual electromagnetic potential and the gauge invariance is then preserved only if $\epsilon A_{\mu} \rightarrow \epsilon A_{\mu}+\lambda_{, \mu}$ for any scalar function $\lambda$. It follows that the the action for the electromagnetic sector is the standard Maxwell action

$$
S_{\epsilon}=-\frac{1}{16 \pi} \int F^{\mu \nu} F_{\mu \nu} \sqrt{-g} \mathrm{~d}^{4} x
$$

for the generalized Faraday tensor

$$
F_{\mu \nu}=\frac{1}{\epsilon}\left[\left(\epsilon A_{\nu}\right)_{, \mu}-\left(\epsilon A_{\mu}\right)_{, \nu} \cdot\right]
$$

To finish the gravitational sector is assumed to be described by the standard Einstein-Hilbert action. Finally, the matter action for point particles of mass $m$ takes the form $S_{m}=\sum \int\left[-m c^{2}+\right.$ $\left.(e / c) u^{\mu} A_{\mu}\right] \gamma^{-1} \delta^{3}\left(x^{i}-x^{i}(\tau)\right) \mathrm{d}^{4} \mathbf{x}$ where $\gamma$ is the Lorentz factor and $\tau$ the proper time. Note that the Maxwell equation becomes

$$
\nabla_{\mu}\left(\epsilon^{-1} F^{\mu \nu}\right)=4 \pi j^{\nu},
$$

which is the same as for electromagnetism in a material medium with dielectric constant $\epsilon^{-2}$ and permeability $\epsilon^{2}$ (this was the original description proposed by Fierz [195] and Lichnerowicz [332]; see also Dicke [152]).

It was proposed [445] to rewrite this theory by introducing the two fields

$$
a_{\mu} \equiv \epsilon A_{\mu}, \quad \psi \equiv \ln \epsilon
$$

so that the theory takes the form

$$
S=\frac{c^{3}}{16 \pi g} \int R \sqrt{-g} \mathrm{~d}^{4} x-\frac{1}{16 \pi} \int \mathrm{e}^{-2 \psi} f^{\mu \nu} f_{\mu \nu} \sqrt{-g} \mathrm{~d}^{4} x-\frac{1}{8 \pi \kappa^{2}} \int\left(\partial_{\mu} \psi\right)^{2} \sqrt{-g} \mathrm{~d}^{4} x
$$

with $\kappa=l /(4 \pi \hbar c)$ and $f_{\mu \nu}$ the Faraday tensor associated with $a_{\mu}$. The model was further extended to include a potential for $\psi$ [32] and to include the electroweak theory [461].

As discussed previously, this class of models predict a violation of the universality of free fall and, from Equation (14), it is expected that the anomalous acceleration is given by $\delta \mathbf{a}=$ $-M^{-1}\left(\partial E_{\mathrm{EM}} / \partial \epsilon\right) \nabla \epsilon$

From the confrontation of the local and cosmological constraints on the variation of $\epsilon$ Bekenstein [39] concluded, given his assumptions on the couplings, that $\alpha_{\mathrm{EM}}$ "is a parameter, not a dynamical variable" (see, however, [40] and then [301]). This problem was recently bypassed by Olive and Pospelov [397] who generalized the model to allow additional coupling of a scalar field $\epsilon^{-2}=B_{F}(\phi)$ to non-baryonic dark matter (as first proposed in [126]) and cosmological constant, arguing that in supersymmetric dark matter, it is natural to expect that $\phi$ would couple more 
strongly to dark matter than to baryon. For instance, supersymmetrizing Bekenstein model, $\phi$ will get a coupling to the kinetic term of the gaugino of the form $M_{*}^{-1} \phi \bar{\chi} \partial \chi$. Assuming that the gaugino is a large fraction of the stable lightest supersymmetric particle, the coupling to dark matter would then be of order $10^{3}-10^{4}$ times larger. Such a factor could almost reconcile the constraint arising from the test of the universality of free fall with the order of magnitude of the cosmological variation. This generalization of the Bekenstein model relies on an action of the form

$$
\begin{aligned}
S & =\frac{1}{2} M_{4}^{2} \int R \sqrt{-g} \mathrm{~d}^{4} \mathbf{x}-\int\left[\frac{1}{2} M_{*}^{2} \partial_{\mu} \phi \partial^{\mu} \phi+\frac{1}{4} B_{F}(\phi) F_{\mu \nu} F^{\mu \nu}\right] \sqrt{-g} \mathrm{~d}^{4} \mathbf{x} \\
& -\int\left\{\sum \bar{N}_{i}\left[i \not D-m_{i} B_{N_{i}}(\phi)\right] N_{i}+\frac{1}{2} \bar{\chi} \partial \chi+M_{4}^{2} B_{\Lambda}(\phi) \Lambda+\frac{1}{2} M_{\chi} B_{\chi}(\phi) \chi^{T} \chi\right\} \sqrt{-g} \mathrm{~d}^{4} \mathbf{x}
\end{aligned}
$$

where the sum is over proton $\left[\not D=\gamma^{\mu}\left(\partial_{\mu}-i e_{0} A_{\mu}\right)\right]$ and neutron $\left[\not D=\gamma^{\mu} \partial_{\mu}\right]$. The functions $B$ can be expanded (since one focuses on small variations of the fine-structure constant and thus of $\phi)$ as $B_{X}=1+\zeta_{X} \phi+\xi_{X} \phi^{2} / 2$. It follows that $\alpha_{\mathrm{EM}}(\phi)=e_{0}^{2} / 4 \pi B_{F}(\phi)$ so that $\Delta \alpha_{\mathrm{EM}} / \alpha_{\mathrm{EM}}=$ $\zeta_{F} \phi+\left(\xi_{F}-2 \zeta_{F}^{2}\right) \phi^{2} / 2$. This framework extends the analysis of [39] to a 4-dimensional parameter space $\left(M_{*}, \zeta_{F}, \zeta_{m}, \zeta_{\Lambda}\right)$. It contains the Bekenstein model $\left(\zeta_{F}=-2, \zeta_{\Lambda}=0, \zeta_{m} \sim 10^{-4} \xi_{F}\right)$, a Jordan-Brans-Dicke model $\left(\zeta_{F}=0, \zeta_{\Lambda}=-2 \sqrt{2 / 2 \omega+3}, \zeta_{m}=-1 / \sqrt{4 \omega+6}\right)$, a string-like model $\left(\zeta_{F}=-\sqrt{2}, \zeta_{\Lambda}=\sqrt{2}, \zeta_{m}=\sqrt{2} / 2\right)$ so that $\left.\Delta \alpha_{\mathrm{EM}} / \alpha_{\mathrm{EM}}=3\right)$ and a supersymmetrized Bekenstein model $\left(\zeta_{F}=-2, \zeta_{\chi}=-2, \zeta_{m}=\zeta_{\chi}\right.$ so that $\left.\Delta \alpha_{\mathrm{EM}} / \alpha_{\mathrm{EM}} \sim 5 / \omega\right)$. In all the models, the universality of free fall sets a strong constraint on $\zeta_{F} / \sqrt{\omega}$ (with $\omega \equiv M_{*} / 2 M_{4}^{2}$ ) and the authors showed that a small set of models may be compatible with a variation of $\alpha_{\mathrm{EM}}$ from quasar data while being compatiblewith the equivalence principle tests. A similar analysis [347] concluded that such models can reproduce the variation of $\alpha_{\mathrm{EM}}$ from quasars while being compatible with Oklo and meteorite data. Note that under this form, the effective theory is very similar to the one detailed in Section 5.4.2.

This theory was also used [41] to study the spacetime structure around charged black-hole, which corresponds to an extension of dilatonic charged black hole. It was concluded that a cosmological growth of $\alpha_{\mathrm{EM}}$ would decrease the black-hole entropy but with half the rate expected from the earlier analysis [139, 339].

\subsubsection{Other ideas}

Let us mention without details other theoretical models, which can accommodate varying constants:

- Models involving a late time phase transition in the electromagnetic sector [87, 10];

- Braneworld models [336, 8, 73, 331, 403] or extra-dimensions [477];

- Model with pseudo-scalar couplings [203];

- Growing neutrino models $[9,533]$ in which the neutrino masses are a function of a scalar field, that is also responsible for the late time acceleration of the universe. In these models the neutrinos freeze the evolution of the scalar field when they become non-relativistic while its evolution is similar as in quintessence when the neutrinos are ultra-relativistic;

- Models based on discrete quantum gravity [223] or on loop quantum gravity in which the Barbero-Immirzi parameter controls the minimum eigenvalue of the area operator and could be promoted to a field, leading to a classical coupling of Einstein's gravity with a scalar-field stress-energy tensor [354, 483]

- "varying speed of light" models for which we refer to the review [341] and our previous analysis [183] for a critical view; 
- Quintessence models with a non-minimal coupling of the quintessence field [20, 11, 96, 112, 162, 217, 315, 314, 389, 347, 404, 531] [see discussion Section 2.2.3];

- Mass varying neutrinos $[9,533]$;

- Holographic dark energy models with non-minimal couplings [235] 


\section{Spatial Variations}

The constraints on the variation of the fundamental constant that we have described so far are mainly related to their cosmological evolution so that, given the Copernican principle, they reduce to constrains on the time variation of the fundamental constants. Indeed, spatial variations can also occur. They may be used to set constraints in two regimes:

- On cosmological scales, the fields dictating the variation of the constants have fluctuations that can let their imprint in some cosmological observables.

- On local scales (e.g., our solar system or the Milky Way) the fields at the origin of the variation of the constants are sourced by the local matter distribution so that one expect that the constants are not homogeneous on these scales.

\subsection{Local scales}

In order to determine the profile of the constant in the solar system, let us assume that their value is dictated by the value of a scalar field. As in Section 5.4.1, we can assume that at lowest order the profile of the scalar field will be obtained from the scalar-tensor theory, taking into account that all masses scale as $\Lambda_{\mathrm{QCD}}\left(\phi_{*}\right)$ where $\phi_{*}$ is the value of the field in the Einstein frame.

\subsubsection{Generalities}

We restrict to the weakly self-gravitating $\left(V_{*} / c^{2} \ll 1\right)$ and slow moving $\left(T^{01} \ll T^{00}\right)$ localized material systems and follow [124]. Using harmonic coordinates, defined with respect to the metric $g_{*}$, the Einstein frame metric can be expanded as

$g_{00}^{*}=-\exp \left(-2 \frac{V_{*}}{c^{2}}\right)+\mathcal{O}\left(c^{-6}\right), \quad g_{0 i}^{*}=-\frac{4}{c^{3}} V_{i}^{*}+\mathcal{O}\left(c^{-5}\right), \quad g_{i j}^{*}=-\exp \left(2 \frac{V_{*}}{c^{2}}\right) \delta_{i j}+\mathcal{O}\left(c^{-6}\right)$,

so that Eqs. (162-163) take the form

$$
\square_{*} V_{*}=-4 \pi G_{*} \sigma_{*}+\mathcal{O}\left(c^{-4}\right), \quad \square_{*} V_{*}^{i}=-4 \pi G_{*} \sigma_{*}^{i}+\mathcal{O}\left(c^{-2}\right), \quad \square_{*} \phi_{*}=-4 \pi G_{*} \frac{S}{c^{2}}+\mathcal{O}\left(c^{-6}\right)
$$

where $\square_{*}$ is the flat d'Alembertian and where the scalar field has been assumed to be light so that one can neglect its potential. The source terms can be expressed in terms of the matter stress-energy tensor in the Einstein frame as

$$
\sigma_{*} c^{2}=T_{*}^{00}+T_{*}^{i i}, \quad \sigma_{*}^{i}=T_{*}^{0 i}, \quad S c^{2}=-\alpha\left(\phi_{*}\right)\left(T_{*}^{00}-T_{*}^{i i}\right) .
$$

Restricting to the static case with a single massive point source, the only non-vanishing source terms are $\sigma_{*}(\mathbf{r})=M_{*} \delta^{3}\left(\mathbf{r}_{*}\right)$ and $S(\mathbf{r})=-\alpha\left(\phi_{*}\right) M_{*} \delta^{3}\left(\mathbf{r}_{*}\right)$ so that the set of equations reduces to two Poisson equations

$$
\Delta_{*} V_{*}=-4 \pi G_{*} M_{*} \delta^{3}\left(\mathbf{r}_{*}\right)+\mathcal{O}\left(c^{-4}\right), \quad \Delta_{*} \phi_{*}=4 \pi \frac{G_{*} M_{*}}{c^{2}} \delta^{3}\left(\mathbf{r}_{*}\right)+\mathcal{O}\left(c^{-6}\right) .
$$

This set of equations can be solved by means of the retarded Green function. It follows that the Einstein frame gravitational potential is $V_{*}\left(r_{*}\right)=G_{*} M_{*} / r_{*}$. The equation for $\phi_{*}$ can be solved iteratively, since at lowest order in $G_{*} / c^{2}$ it has solution

$$
\phi_{*}=\phi_{1}\left(r_{*}\right) \equiv \phi_{0}-\frac{\alpha_{0}}{c^{2}} V_{*}\left(r_{*}\right) .
$$


This can be used to determine the Jordan frame metric and the variation of the scalar field in function of the Jordan frame coordinates. It follows that at lowest order the Newton potential and the scalar field are given by

$$
\Phi_{N}=\frac{G M}{r}, \quad \phi_{*}=\phi_{1}(r) \equiv \phi_{0}-\alpha_{0} \frac{\Phi_{N}(r)}{c^{2}},
$$

where we have neglected the corrections $-\alpha(\phi)\left(\phi-\phi_{0}\right)$ for the gravitational potential, which, given the solar system constraints on $\alpha_{0}$, is a good approximation.

Now let us consider any constant $\alpha_{i}$ function of $\phi$. Thus, its profile is given by $\alpha_{i}(r)=$ $\alpha_{i}\left(\phi_{0}\right)-\alpha_{0} \alpha_{i}^{\prime}\left(\phi_{0}\right) \Phi_{N}(r) / c^{2}$ so that

$$
\frac{\Delta \alpha_{i}}{\alpha_{i}}(r)=-s_{i}\left(\phi_{0}\right) \alpha_{0} \frac{\Phi_{N}(r)}{c^{2}}
$$

where $s_{i}\left(\phi_{0}\right)$ is the sensitivity of the constant $\alpha_{i}$ to a variation of the scalar field, $s_{i} \equiv \mathrm{d} \ln \alpha_{i} / \mathrm{d} \phi$. For laboratory in orbit on an elliptic trajectory,

$$
r=\frac{a\left(1-e^{2}\right)}{1+e \cos \psi}, \quad \cos \psi=\frac{\cos E-e}{1-e \cos E}, \quad t=\sqrt{\frac{a^{3}}{G M}}(E-e \sin E)
$$

where $a$ is the semi-major axis, $e$ the eccentricity and $\psi$ the true anomaly. It follows that

$$
\frac{\Delta \alpha_{i}}{\alpha_{i}}(a, \psi)=-s_{0} \alpha_{0} \frac{G M}{a c^{2}}-s_{0} \alpha_{0} \frac{G M}{a c^{2}} e \cos \psi+\mathcal{O}\left(e^{2}\right) .
$$

The first term represents the variation of the mean value of the constant on the orbit compared with its cosmological value. This shows that local terrestrial and solar system experiments do measure the effects of the cosmological variation of the constants [124, 461, 460, 462]. The second term is a seasonal modulation and it is usually parameterized [209] as

$$
\left.\frac{\Delta \alpha_{i}}{\alpha_{i}}\right|_{\text {seasonal }}=k_{i} \frac{\Delta \Phi_{N}}{c^{2}}
$$

defining the parameters $k_{i}$.

\subsubsection{Solar system scales}

The parameters $k_{i}$ can be constrained from laboratory measurements on Earth. Since $e \simeq 0.0167$ for the Earth orbit, the signal should have a peak-to-peak amplitude of $2 \mathrm{GMe} / a c^{2} \sim 3.3 \times 10^{-10}$ on a period of 1 year. This shows that the order of magnitude of the constraints will be roughly of $10^{-16} / 10^{-10} \sim 10^{-6}$ since atomic clocks reach an accuracy of the order of $10^{-16}$. The data of [214] and [37] lead respectively to the two constraints [209]

$$
k_{\alpha_{\mathrm{EM}}}+0.17 k_{e}=(-3.5 \pm 6) \times 10^{-7}, \quad\left|k_{\alpha_{\mathrm{EM}}}+0.13 k_{e}\right|<2.5 \times 10^{-5},
$$

for $\alpha_{\mathrm{EM}}$ and $m_{\mathrm{e}} / \Lambda_{\mathrm{QCD}}$ respectively. The atomic dysprosium experiment [100] allowed to set the constraint [193]

$$
k_{\alpha_{\mathrm{EM}}}=(-8.7 \pm 6.6) \times 10-6,
$$

which, combined with the previous constraints, allows to conclude that

$$
k_{e}=(4.9 \pm 3.9) \times 10^{-5}, \quad k_{q}=(6.6 \pm 5.2) \times 10^{-5}
$$


for $m_{\mathrm{e}} / \Lambda_{\mathrm{QCD}}$ and $m_{\mathrm{q}} / \Lambda_{\mathrm{QCD}}$ respectively. [61], using the comparison of cesium and a strontium clocks derived that

$$
k_{\alpha_{\mathrm{EM}}}+0.36 k_{e}=(1.8 \pm 3.2) \times 10^{-5},
$$

which, combined with measurement of H-maser [17], allow one to set the three constraints as

$$
k_{\alpha_{\mathrm{EM}}}=(2.5 \pm 3.1) \times 10^{-6}, \quad k_{\mu}=(-1.3 \pm 1.7) \times 10^{-5}, \quad k_{q}=(-1.9 \pm 2.7) \times 10^{-5} .
$$

$[34,463]$ reanalyzed the data by [408] to conclude that $k_{\alpha_{\mathrm{EM}}}+0.51 k_{\mu}=(7.1 \pm 3.4) \times 10^{-6}$. Combined with the constraint (218), it led to

$$
k_{\mu}=(3.9 \pm 3.1) \times 10^{-6}, \quad k_{q}=(0.1 \pm 1.4) \times 10^{-5} .
$$

[34] also used the data of [440] to conclude

$$
k_{\alpha_{\mathrm{EM}}}=(-5.4 \pm 5.1) \times 10^{-8} .
$$

All these constraints use the sensitivity coefficients computed in $[14,210]$. We refer to [265] as an unexplained seasonal variation that demonstrated the difficulty to interpret phenomena.

Such bounds can be improved by comparing clocks on Earth and onboard of satellites [209, $444,343]$ while the observation of atomic spectra near the Sun can lead to an accuracy of order unity [209]. A space mission with atomic clocks onboard and sent to the Sun could reach an accuracy of $10^{-8}[343,547]$.

\subsubsection{Milky Way}

An attempt $[323,358]$ to constrain $k_{\mu}$ from emission lines due to ammonia in interstellar clouds of the Milky Way led to the conclusion that $k_{\mu} \sim 1$, by considering different transitions in different environments. This is in contradiction with the local constraint (219). This may result from rest frequency uncertainties or it would require that a mechanism such as chameleon is at work (see Section 5.4.2) in order to be compatible with local constraints. The analysis was based on an ammonia spectra atlas of 193 dense protostellar and prestellar cores of low masses in the Perseus molecular cloud, comparison of $\mathrm{N}_{2} \mathrm{H}^{+}$and $\mathrm{N}_{2} \mathrm{D}^{+}$in the dark cloud L183.

A second analysis [324] using high resolution spectral observations of molecular core in lines of $\mathrm{NH}_{3}, \mathrm{HC}_{3} \mathrm{~N}$ and $\mathrm{N}_{2} \mathrm{H}^{+}$with 3 radio-telescopes showed that $|\Delta \mu / \mu|<3 \times 10^{-8}$ between the cloud environment and the local laboratory environment. However, an offset was measured that could be interpreted as a variation of $\mu$ of amplitude $\Delta \bar{\mu} / \bar{\mu}=\left(2.2 \pm 0.4_{\text {stat }} \pm 0.3_{\text {sys }}\right) \times 10^{-8}$. A second analysis [322] map four molecular cores L1498, L1512, L1517, and L1400K selected from the previous sample in order to estimate systematic effects due to possible velocity gradients. The measured velocity offset, once expressed in terms of $\Delta \bar{\mu}$, gives $\Delta \bar{\mu}=\left(26 \pm 1_{\text {stat }} \pm 3_{\text {sys }}\right) \times 10^{-9}$.

A similar analysis [326] based on the fine-structure transitions in atomic carbon $\mathrm{C}$ I and lowlaying rotational transitions in ${ }^{13} \mathrm{CO}$ probed the spatial variation of $F=\alpha_{\mathrm{EM}}^{2} \mu$ over the galaxy. It concluded that

$$
\left|\Delta F^{\prime} / F^{\prime}\right|<3.7 \times 10^{-7}
$$

between high (terrestrial) and low (interstellar) densities of baryonic matter. Combined with the previous constraint on $\mu$ it would imply that $\left|\Delta \alpha_{\mathrm{EM}} / \alpha_{\mathrm{EM}}\right|<2 \times 10^{-7}$. This was updated [319] to $\left|\Delta F^{\prime} / F^{\prime}\right|<2.3 \times 10^{-7}$ so that $\left|\Delta \alpha_{\mathrm{EM}} / \alpha_{\mathrm{EM}}\right|<1.1 \times 10^{-7}$.

Since extragalactic gas clouds have densities similar to those in the interstellar medium, these bounds give an upper bound on a hypothetic chameleon effect, which are much below the constraints obtained on time variations from QSO absorption spectra. 


\subsection{Cosmological scales}

During inflation, any light scalar field develop super-Hubble fluctuations of quantum origin, with an almost scale invariant power spectrum (see chapter 8 of [409]). It follows that if the fundamental constants depend on such a field, their value must fluctuate on cosmological scales and have a non-vanishing correlation function. More important these fluctuations can be correlated with the metric perturbations.

In such a case, the fine-structure constant will behave as $\alpha_{\mathrm{EM}}=\alpha_{\mathrm{EM}}(t)+\delta \alpha_{\mathrm{EM}}(\mathbf{x}, t)$, the fluctuations being a stochastic variable. As we have seen earlier, $\alpha_{\mathrm{EM}}$ enters the dynamics of recombination, which would then become patchy. This has several consequences for the CMB anisotropies. In particular, similarly to weak gravitational lensing, it will modify the mean power spectra (this is a negligible effect) and induce a curl component (B mode) to the polarization [466]. Such spatial fluctuations also induce non-Gaussian temperature and polarization correlations in the CMB [466, 417]. Such correlations have not allowed to set observational constraints yet but they need to be included foe consistency, see e.g., the example of CMB computation in scalartensor theories [435]. The effect on large the scale structure was also studied in [30, 363] and the Keck/HIRES QSO absorption spectra showed [377] that the correlation function of the finestructure constant is consistent on scales ranging between 0.2 and $13 \mathrm{Gpc}$.

Recently, it has been claimed $[50,523]$ that the fine structure constant may have a dipolar variation that would explain consistently the data from the Southern and Northern hemispheres (see Section 3.4.3). Let assume a constant, $X$ say, depend on the local value of a dynamical scalar field $\phi$. The value of $X$ at the observation point is compared to its value here and today,

$$
\Delta X / X_{0} \equiv X(\phi) / X\left(\phi_{0}\right)-1 \text {. }
$$

Decomposing the scalar field as $\phi=\phi_{0}+\Delta \phi$, one gets that $\Delta X / X_{0}=s_{X}(\phi) \Delta \phi$, with $s_{X}$ defined in Equation (233). Now the scalar field can be decomposed into a background and perturbations as $\phi=\bar{\phi}(t)+\delta \phi(\mathbf{x}, t)$ where the background value depends only on $t$ because of the Copernican hypothesis. It follows that

$$
\begin{aligned}
\frac{\Delta X(\mathbf{x}, t)}{X_{0}} & =s_{X}(\bar{\phi})\left[\bar{\phi}(t)-\phi_{0}\right]+\left\{s_{X}(\bar{\phi})+s_{X}^{\prime}(\bar{\phi})\left[\bar{\phi}(t)-\phi_{0}\right]\right\} \delta \phi(\mathbf{x}, t) \\
& \equiv s_{X}(\bar{\phi}) \Delta \bar{\phi}+\mathcal{S}_{X}(\bar{\phi}) \delta \phi(\mathbf{x}, t) .
\end{aligned}
$$

The first term of the r.h.s. depends only on time while the second is space-time dependent. It is also expected that the second term in the curly brackets is negligible with respect to the first, i.e., $\mathcal{S}_{X}(\bar{\phi}) \sim s_{X}(\bar{\phi})$. It follows that one needs $\delta \phi(\mathbf{x}, t)$ not to be small compared to the background evolution term $\Delta \bar{\phi}$ for the spatial dependence to dominate over the large scale time dependence. This can be achieved for instance if $\phi$ is a seed field whose mean value is frozen. Because of statistical isotropy, and in the same way as for CMB anisotropies (see, e.g., [409]), one can express the equal-time angular power spectrum of $\Delta X / X_{0}$ for two events on our past lightcone as

$$
\left\langle\frac{\Delta X\left(\mathbf{n}_{1}, r, t\right)}{X_{0}} \frac{\Delta X\left(\mathbf{n}_{2}, r, t\right)}{X_{0}}\right\rangle=\sum_{\ell} \frac{2 \ell+1}{4 \pi} C_{\ell}^{(X X)}(z) P_{\ell}\left(\mathbf{n}_{1} \cdot \mathbf{n}_{2}\right) .
$$

If $\delta \phi$ is a stochastic field characterized by its power spectrum, $\left\langle\delta \phi\left(\mathbf{k}_{1}, t\right) \delta \phi\left(\mathbf{k}_{2}, t\right)\right\rangle=P_{\phi}(k, t) \delta\left(\mathbf{k}_{1}+\right.$ $\mathbf{k}_{2}$ ) in Fourier space, then

$$
C_{\ell}^{(X X)}(z)=\frac{2}{\pi} \mathcal{S}_{X}^{2}[\bar{\phi}(z)] \int P_{\phi}(k, z) j_{\ell}\left[k\left(\eta_{0}-\eta\right)\right] k^{2} \mathrm{~d} k,
$$

$j_{\ell}$ being a spherical Bessel function. For instance, if $P_{\phi} \propto k^{n_{s}-1}$ where $n_{s}$ is a spectral index, $n_{s}=1$ corresponding to scale invariance, one gets that $\ell(\ell+1) C_{\ell}^{(X X)} \propto \ell^{n_{s}-1}$ on large angular scales.

Living Reviews in Relativity

http://www. livingreviews . org//rr-2011-2 
The comparison of the amplitude of the angular correlation and the isotropic (time) variation is model-dependent and has not yet been investigated. It was suggested that a spatial variation of $\alpha_{\mathrm{EM}}$ would induce a dipolar modulation of CMB anisotropies [362], but at a level incompatible with existing constraints [424].

This has lead to the idea [396] of the existence of a low energy domain wall produced in the spontaneous symmetry breaking involving a dilaton-like scalar field coupled to electromagnetism. Domains on either side of the wall exhibit slight differences in their respective values of $\alpha_{\mathrm{EM}}$. If such a wall is present within our Hubble volume, absorption spectra at large redshifts may or may not provide a variation in $\alpha_{\mathrm{EM}}$ relative to the terrestrial value, depending on our relative position with respect to the wall.

Another possibility would be that the Copernican principle is not fully satisfied, such as in various void models. Then the background value of $\phi$ would depend, e.g., on $r$ and $t$ for a spherically symmetric spacetime (such as a Lemaitre-Tolman-Bondi spacetime). This could give rise to a dipolar modulation of the constant if the observer (us) is not located at the center of the universe. Note, however, that such a cosmological dipole would also reflect itself, e.g., on CMB anisotropies. Similar possibilities are also offered within the chameleon mechanism where the value of the scalar field depends on the local matter density (see Section 5.4.2).

More speculative, is the effect that such fluctuations can have during preheating after inflation since the decay rate of the inflaton in particles may fluctuate on large scales [293, 294].

\subsection{Implication for the universality of free fall}

As we have seen in the previous sections, the tests of the universality of free fall is central in containing the model involving variations of the fundamental constants.

From Equation (14), the amplitude of the violation of the universality of free fall is given by $\eta_{A B}$, which takes the form

$$
\eta_{A B}=\frac{1}{g_{N}} \sum_{i}\left|f_{A i}-f_{B i}\right|\left|\nabla \alpha_{i}\right| .
$$

In the case in which the variation of the constants arises from the same scalar field, the analysis of Section 6.1 implies that $\nabla \alpha_{i}$ can be related to the gravitational potential by $\left|\nabla \alpha_{i}\right|=\alpha_{i} s_{i}(\phi) \alpha_{\text {ext }} g_{N}$ so that

$$
\eta_{A B}=\sum_{i}\left|f_{A i}-f_{B i}\right| s_{i}(\phi) \alpha_{i} \alpha_{\mathrm{ext}}=\sum_{i}\left|\lambda_{A i}-\lambda_{B i}\right| s_{i}(\phi) \alpha_{\mathrm{ext}} .
$$

This can be expressed in terms of the sensitivity coefficient $k_{i}$ defined in Equation (214) as

$$
\eta_{A B}=\sum_{i}\left|\lambda_{A i}-\lambda_{B i}\right| k_{i}
$$

since $\left|\nabla \alpha_{i}\right|=\alpha_{i} k_{i} g_{N}$. This shows that each experiment will yield a constraint on a linear combination of the coefficients $k_{i}$ so that one requires at least as many independent pairs of test bodies as the number of constants to be constrained.

While the couplings to mass number, lepton number and the electromagnetic binding energy have been considered [118] [see the example of Section 5.4.1] the coupling to quark masses remains a difficult issue. In particular, the whole difficulty lies in the determination of the coefficients $\lambda_{a i}$ [see Section 5.3.2]. In the formalism developed in [122, 121], see Section 5.4.1, one can relate the expected deviation from the universality of free fall to the 5 parameters $d$ and get constraints on $D_{\hat{m}} \equiv d_{g}^{*}\left(d_{\hat{m}}-d_{g}\right)$ and $D_{e} \equiv d_{g}^{*} d_{e}$ where $d_{g}^{*} \equiv d_{g}+0.093\left(d_{\hat{m}}-d_{g}\right)+0.00027 d_{e}$. For instance, the Be-Ti EötWash experiment and LRR experiment respectively imply

$$
\left|D_{\hat{m}}+0.22 D_{e}\right|<5.1 \times 10^{-11}, \quad\left|D_{\hat{m}}+0.28 D_{e}\right|<24.6 \times 10^{-11} .
$$


This shows that while the Lunar experiment has a slightly better differential-acceleration sensitivity, the laboratory-based test is more sensitive to the dilaton coefficients because of a greater difference in the dilaton charges of the materials used, and of the fact that only one-third of the Earth mass is made of a different material.

The link between the time variation of fundamental constants and the violation of the universality of free fall have been discussed by Bekenstein [39] in the framework described in Section 5.4.2 and by Damour-Polyakov [135, 136] in the general framework described in Section 5.4.1. In all these models, the two effects are triggered by a scalar field. It evolves according to a Klein-Gordon equation $\left(\ddot{\phi}+3 H \dot{\phi}+m^{2} \phi+\ldots=0\right)$, which implies that $\phi$ is damped as $\dot{\phi} \propto a^{-3}$ if its mass is much smaller than the Hubble scale. Thus, in order to be varying during the last Hubble time, $\phi$ has to be very light with typical mass $m \sim H_{0} \sim 10^{-33} \mathrm{eV}$. As a consequence, $\phi$ has to be very weakly coupled to the standard model fields to avoid a violation of the universality of free fall.

This link was revisited in $[96,166,532]$ in which the dependence of $\alpha_{\mathrm{EM}}$ on the scalar field responsible for its variation is expanded as

$$
\alpha_{\mathrm{EM}}=\alpha_{\mathrm{EM}}(0)+\lambda \frac{\phi}{M_{4}}+\mathcal{O}\left(\frac{\phi^{2}}{M_{4}^{2}}\right) .
$$

The cosmological observation from QSO spectra implies that $\lambda \Delta \phi / M_{4} \sim 10^{-7}$ at best during the last Hubble time. Concentrating only on the electromagnetic binding energy contribution to the proton and of the neutron masses, it was concluded that a test body composed of $n_{\mathrm{n}}$ neutrons and $n_{\mathrm{p}}$ protons will be characterized by a sensitivity $\lambda\left(\nu_{\mathrm{p}} B_{\mathrm{p}}+\nu_{\mathrm{n}} B_{\mathrm{n}}\right) / m_{\mathrm{N}}$ where $\nu_{\mathrm{n}}$ (resp. $\nu_{\mathrm{p}}$ ) is the ratio of neutrons (resp. protons) and where it has been assumed that $m_{\mathrm{n}} \sim$ $m_{\mathrm{p}} \sim m_{\mathrm{N}}$. Assuming ${ }^{13}$ that $\nu_{\mathrm{n}, \mathrm{p}}^{\text {Earth }} \sim 1 / 2$ and using that the compactness of the Moon-Earth system $\partial \ln \left(m_{\text {Earth }} / m_{\text {Moon }}\right) / \partial \ln \alpha_{\mathrm{EM}} \sim 10^{-3}$, one gets $\eta_{12} \sim 10^{-3} \lambda^{2}$. Dvali and Zaldarriaga [166] obtained the same result by considering that $\Delta \nu_{\mathrm{n}, \mathrm{p}} \sim 6 \times 10^{-2}-10^{-1}$. This implies that $\lambda<10^{-5}$, which is compatible with the variation of $\alpha_{\mathrm{EM}}$ if $\Delta \phi / M_{4}>10^{-2}$ during the last Hubble period. From the cosmology one can deduce that $\left(\Delta \phi / M_{4}\right)^{2} \sim\left(\rho_{\phi}+P_{\phi}\right) / \rho_{\text {total }}$. If $\phi$ dominates the matter content of the universe, $\rho_{\text {total }}$, then $\Delta \phi \sim M_{4}$ so that $\lambda \sim 10^{-7}$ whereas if it is sub-dominant $\Delta \phi \ll M_{4}$ and $\lambda \gg 10^{-7}$. In conclusion $10^{-7}<\lambda<10^{-5}$. This makes explicit the tuning of the parameter $\lambda$. Indeed, an important underlying approximation is that the $\phi$-dependence arises only from the electromagnetic self-energy. This analysis was extended in [143] who included explicitly the electron and related the violation of the universality of free fall to the variation of $\mu$.

In a similar analysis [532], the scalar field is responsible for both a variation of $\alpha_{\mathrm{EM}}$ and for the acceleration of the universe. Assuming its equation of state is $w_{h} \neq 1$, one can express its time variation (as long as it has a standard kinetic term) as

$$
\dot{\phi}=H \sqrt{3 \Omega_{\phi}\left(1+w_{h}\right)} \text {. }
$$

It follows that the expected violation of the universality of free fall is related to the time variation of $\alpha_{\mathrm{EM}}$ today by

$$
\eta=-1.75 \times 10^{-2}\left(\frac{\partial \ln \alpha_{\mathrm{EM}}}{\partial z}\right)_{z=0}^{2} \frac{(1+\tilde{Q}) \Delta \frac{Z}{Z+N}}{\Omega_{\phi}^{(0)}\left(1+w_{h}^{(0)}\right)},
$$

where $\tilde{Q}$ is a parameter taking into account the influence of the mass ratios. Again, this shows that in the worse case in which the Oklo bound is saturated (so that $\partial \ln \alpha_{\mathrm{EM}} / \partial z \sim 10^{-6}$ ), one requires $1+w_{h}^{(0)} \gtrsim 10^{-2}$ for $\eta \lesssim 10^{-13}$, hence providing a string bond between the dark energy equation of state and the violation of the universality of free fall. This was extended in [149] in terms of the phenomenological model of unification presented in Section 5.3.1. In the case of the

\footnotetext{
13 For copper $\nu_{\mathrm{p}}=0.456$, for uranium $\nu_{\mathrm{p}}=0.385$ and for lead $\nu_{\mathrm{p}}=0.397$.
} 
string dilaton and runaway dilaton models, one reaches a similar conclusion [see Equation (203) in Section 5.4.1]. A similar result [348] was obtained in the case of pure scalar-tensor theory, relating the equation of state to the post-Newtonian parameters. In all these models, the link between the local constraints and the cosmological constraints arise from the fact that local experiments constrain the upper value of $\dot{\phi}_{0}$, which quantify both the deviation of its equation of state from -1 and the variation of the constants. It was conjectured that most realistic quintessence models suffer from such a problem [68].

One question concerns the most sensitive probes of the equivalence principle. This was investigated in [144] in which the coefficients $\lambda_{A i}$ are estimated using the model (189). It was concluded that they are $2-3$ orders of magnitude over cosmic clock bounds. However, [148] concluded that the most sensitive probe depends on the unification relation that exist between the different couplings of the standard model. [463] concluded similarly that the universality of free fall is more constraining that the seasonal variations. The comparison with QSO spectra is more difficult since it involves the dynamics of the field between $z \sim 1$ and today. To finish, let us stress that these results may be changed significantly if a chameleon mechanism is at work. 


\section{$7 \quad$ Why Are The Constants Just So?}

The numerical values of the fundamental constants are not determined by the laws of nature in which they appear. One can wonder why they have the values we observe. In particular, as pointed out by many authors (see below), the constants of nature seem to be fine tuned [317]. Many physicists take this fine-tuning to be an explanandum that cries for an explanans, hence following Hoyle [258] who wrote that "one must at least have a modicum of curiosity about the strange dimensionless numbers that appear in physics."

\subsection{Universe and multiverse approaches}

Two possible lines of explanation are usually envisioned: a design or consistency hypothesis and an ensemble hypothesis, that are indeed not incompatible together. The first hypothesis includes the possibility that all the dimensionless parameters in the "final" physical theory will be fixed by a condition of consistency or an external cause. In the ensemble hypothesis, the universe we observe is only a small part of the totality of physical existence, usually called the multiverse. This structure needs not be fine-tuned and shall be sufficiently large and variegated so that it can contain as a proper part a universe like the one we observe the fine-tuning of which is then explained by an observation selection effect [64].

These two possibilities send us back to the large number hypothesis by Dirac [155] that has been used as an early motivation to investigate theories with varying constants. The main concern was the existence of some large ratios between some combinations of constants. As we have seen in Section 5.3.1, the running of coupling constants with energy, dimensional transmutation or relations such as Equation (185) have opened a way to a rational explanation of very small (or very large) dimensional numbers. This follows the ideas developed by Eddington [178, 179] aiming at deriving the values of the constants from consistency relations, e.g., he proposed to link the fine-structure constant to some algebraic structure of spacetime. Dicke [151] pointed out another possible explanation to the origin of Dirac large numbers: the density of the universe is determined by its age, this age being related to the time needed to form galaxies, stars, heavy nuclei... This led Carter [82] to argue that these numerical coincidence should not be a surprise and that conventional physics and cosmology could have been used to predict them, at the expense of using the anthropic principle.

The idea of such a structure called the multiverse has attracted a lot of attention in the past years and we refer to [79] for a more exhaustive account of this debate. While many versions of what such a multiverse could be, one of them finds its root in string theory. In 2000, it was realized [66] that vast numbers of discrete choices, called flux vacua, can be obtained in compactifying superstring theory. The number of possibilities is estimated to range between $10^{100}$ and $10^{500}$, or maybe more. No principle is yet known to fix which of these vacua is chosen. Eternal inflation offers a possibility to populate these vacua and to generate an infinite number of regions in which the parameters, initial conditions but also the laws of nature or the number of spacetime dimensions can vary from one universe to another, hence being completely contingent. It was later suggested by Susskind [482] that the anthropic principle may actually constrain our possible locations in this vast string landscape. This is a shift from the early hopes [270] that M-theory may conceivably predict all the fundamental constants uniquely.

Indeed such a possibility radically changes the way we approach the question of the relation of these parameters to the underlying fundamental theory since we now expect them to be distributed randomly in some range. Among this range of parameters lies a subset, that we shall call the anthropic range, which allow for universe to support the existence of observers. This range can be determined by asking ourselves how the world would change if the values of the constants were changed, hence doing counterfactual cosmology. However, this is very restrictive since the

Living Reviews in Relativity

http: //www. livingreviews.org/lrr-2011-2 
mathematical form of the law of physics managed as well and we are restricting to a local analysis in the neighborhood of our observed universe. The determination of the anthropic region is not a prediction but just a characterization of the sensitivity of "our" universe to a change of the fundamental constants ceteris paribus. Once this range is determined, one can ask the general question of quantifying the probability that we observe a universe as ours, hence providing a probabilistic prediction. This involves the use of the anthropic principle, which expresses the fact that we observe are not just observations but observations made by us, and requires us to state what an observer actually is [383].

\subsection{Fine-tunings and determination of the anthropic range}

As we have discussed in the previous sections, the outcome of many physical processes are strongly dependent on the value of the fundamental constants. One can always ask the scientific question of what would change in the world around us if the values of some constants were changed, hence doing some counterfactual cosmology in order to determine the range within which the universe would have developed complex physics and chemistry, what is usually thought to be a prerequisit for the emergence of complexity and life (we emphasize the difficulty of this exercise when it goes beyond small and local deviations from our observed universe and physics, see, e.g., [245] for a possibly life supporting universe without weak interaction). In doing so, one should consider the fundamental parameters entering our physical theory but also the cosmological parameters.

First there are several constraints that the fundamental parameters listed in Table 1 have to satisfy in order for the universe to allow for complex physics and chemistry. Let us stress, in a non-limiting way, some examples.

- It has been noted that the stability of the proton requires $m_{\mathrm{d}}-m_{\mathrm{u}} \gtrsim \alpha_{\mathrm{EM}}^{3 / 2} m_{\mathrm{p}}$. The anthropic bounds on $m_{\mathrm{d}}, m_{\mathrm{u}}$ and $m_{\mathrm{e}}$ (or on the Higgs vev) arising from the existence of nuclei, the di-neutron and the di-proton cannot form a bound state, the deuterium is stable have been investigated in many works $[5,6,120,145,160,161,252,254]$, even allowing for nuclei made of more than 2 baryon species [264]. Typically, the existence of nuclei imposes that $m_{\mathrm{d}}+m_{\mathrm{u}}$ and $v$ cannot vary by more that $60 \%$ from their observed value in our universe.

- If the difference of the neutron and proton masses where less that about $1 \mathrm{MeV}$, the neutron would become stable and hydrogen would be unstable $[442,253]$ so that helium would have been the most abundant at the end of BBN so that the whole history of the formation and burning of stars would have been different. It can be deduced that [252] one needs $m_{\mathrm{d}}-m_{\mathrm{u}}-m_{\mathrm{e}} \gtrsim 1.2 \mathrm{MeV}$ so that the universe does not become all neutrons; $m_{\mathrm{d}}-m_{\mathrm{u}}+m_{\mathrm{e}} \lesssim$ $3.4 \mathrm{MeV}$ for the $p p$ reaction to be exothermic and $m_{\mathrm{e}}>0$ leading to a finite domain.

- A coincidence emerges from the existence of stars with convective and radiative envelopes, since it requires [80] that $\alpha_{\mathrm{G}} \sim \alpha_{\mathrm{EM}}^{20}$. It arises from the fact that the typical mass that separates these two behavior is roughly $\alpha_{\mathrm{G}}^{-2} \alpha_{\mathrm{EM}}^{10} m_{\mathrm{p}}$ while the masses of star span a few decades around $\alpha_{\mathrm{G}}^{-3} m_{\mathrm{p}}$. Both stars seem to be needed since only radiative stars can lead to supernovae, required to disseminate heavy elements, while only convective stars may generate winds in their early phase, which may be associated with formation of rocky planets. This relation while being satisfied numerically in our universe cannot be explained from fundamental principles.

- Similarly, it seems that for neutrinos to eject the envelope of a star in a supernovae explosion, one requires [80] $\alpha_{\mathrm{G}} \sim \alpha_{\mathrm{W}}^{4}$.

- As we discussed in Section 3.5, the production of carbon seems to imply that the relative strength of the nuclear to electromagnetic interaction must be tuned typically at the $0.1 \%$ level. 
Other coincidences involve also the physical properties, not only of the physical theories, but also of our universe, i.e., the cosmological parameters summarized in Table 4. Let us remind some examples.

- The total density parameter $\Omega$ must lie within an order of magnitude of unity. If it were much larger the universe will have re-collapsed rapidly, on a time scale much shorter that the main-sequence star lifetime. If it were to small, density fluctuations would have frozen before galaxies could form. Typically one expects $0.1<\Omega_{0}<10$. Indeed, most inflationary scenarios lead to $\Omega_{0} \sim 1$ so that this may not be anthropically determined but in that case inflation should last sufficiently long so that this could lead to a fine tuning on the parameters of the inflationary potential.

- The cosmological constant was probably the first one to be questioned in an anthropical way [527]. Weinberg noted that if $\Lambda$ is too large, the universe will start accelerating before structures had time to form. Assuming that it does not dominate the matter content of the universe before the redshift $z_{*}$ at which earliest are formed, one concludes that $\rho_{V}=$ $\Lambda / 8 \pi G<\left(+z_{*}\right) \rho_{\text {mat } 0}$. Weinberg [527] estimated $z_{*} \sim 4.5$ and concluded that "if it is the anthropic principle that accounts for the smallness of the cosmological constant, then we would expect the vacuum energy density $\rho_{v} \sim(10-100) \rho_{\text {mat0 }}$ because there is no anthropic reason for it to be smaller". Indeed, the observations indicate $\rho_{v} \sim 2 \rho_{\text {mat0 }}$

- Tegmark and Rees [486] have pointed out that the amplitude of the initial density perturbation, $Q$ enters into the calculation and determined the anthropic region in the plane $(\Lambda, Q)$. This demonstrates the importance of determining the parameters to include in the analysis.

- Different time scales of different origin seem to be comparable: the radiative cooling, galactic halo virialization, time of cosmological constant dominance, the age of the universe today. These coincidence were interpreted as an anthropic sign [65].

These are just a series of examples. For a multi-parameter study of the anthropic bound, we refer, e.g., to [485] and to [243] for a general anthropic investigation of the standard model parameters.

\subsection{Anthropic predictions}

The determination of the anthropic region for a set of parameters is in no way a prediction but simply a characterization of our understanding of a physical phenomenon $P$ that we think is important for the emergence of observers. It reflects that, the condition $C$ stating that the constants are in some interval, $C \Longrightarrow P$, is equivalent to $! P \Longrightarrow ! C$.

The anthropic principle [82] states that "what we can expect to observe must be restricted by the conditions necessary for our presence as observers". It has received many interpretations among which the weak anthropic principle stating that "we must be prepared to take account of the fact that our location in the universe in necessarily privileged to the extent of being compatible with our existence as observers", which is a restriction of the Copernican principle often used in cosmology, and the strong anthropic principle according to which "the universe (and hence the fundamental parameters on which it depends) must be such as to admit the creation of observers within it at some stage." (see [35] for further discussions and a large bibliography on the subject).

One can then try to determine the probability that an observer measure the value $x$ of the constant $X$ (that is a random variable fluctuating in the multiverse and the density of observers depend on the local value of $X$ ). According to Bayes theorem,

$$
P(X=x \mid \mathrm{obs}) \propto P(\mathrm{obs} \mid X=x) P(X=x) .
$$

Living Reviews in Relativity

http: //www . livingreviews . org/lrr-2011-2 
$P(X=x)$ is the prior distribution, which is related to the volume of those parts of the universe in which $X=x$ at $\mathrm{d} x . P(\operatorname{obs} \mid X=x)$ is proportional to the density of observers that are going to evolve when $X=x . P(X=x \mid$ obs $)$ then gives the probability that a randomly selected observer is located in a region where $X=x \pm \mathrm{d} x$. It is usually rewritten as [519]

$$
P(x) \mathrm{d} x=n_{\text {obs }}(x) P_{\text {prior }} \mathrm{d} x .
$$

This higlights the difficulty in making a prediction. First, one has no idea of how to compute $n_{\text {obs }}(x)$. When restricting to the cosmological constant, one can argue [519] that $\Lambda$ does not affect microphysics and chemistry and then estimate $n_{\text {obs }}(x)$ by the fraction of matter clustered in giant galaxies and that can be computed from a model of structure formation. This may not be a good approximation when other constants are allowed to vary and it needs to be defined properly. Second, $P_{\text {prior }}$ requires an explicit model of multiverse that would generate sub-universes with different values $x_{i}$ (continuous or discrete) for $x$. A general argument [528] states that if the range over which $X$ varies in the multiverse is large compared to the anthropic region $X \in\left[X_{\min }, X_{\max }\right]$ one can postulate that $P_{\text {prior }}$ is flat on $\left[X_{\min }, X_{\max }\right]$. Indeed, such a statement requires a measure in the space of the constants (or of the theories) that are allowed to vary. This is a strong hypothesis, which is difficult to control. In particular if $P_{\text {prior }}$ peaks outside of the anthropic domain, it would predict that the constants should lie on the boundary of the anthropic domain [443]. It also requires that there are sufficiently enough values of $x_{i}$ in the anthropic domain, i.e., $\delta x_{i} \ll X_{\max }-X_{\min }$. Garriga and Vilenkin [228] stressed that the hypothesis of a flat $P_{\text {prior }}$ for the cosmological constant may not hold in various Higgs models, and that the weight can lower the mean viable value. To finish, one want to consider $P(x)$ as the probability that a random observer measures the value $x$. This relies on the fact that we are a typical observer and we are implicitly making a self sampling hypothesis. It requires to state in which class of observers we are supposed to be typical (and the final result may depend on this choice [383]) and this hypothesis leads to conclusions such as the doomsday argument that have be debated actively [64, 383].

This approach to the understanding of the observed values of the fundamental constants (but also of the initial conditions of our universe) by resorting to the actual existence of a multiverse populated by a different "low-energy" theory of some "mother" microscopic theory allows us to explain the observed fine-tuning by an observational selection effect. It also sets a limit to the Copernican principle stating that we do not live in a particular position in space since we have to live in a region of the multiverse where the constants are inside the anthropic bound. Such an approach is indeed not widely accepted and has been criticized in many ways [7, 182, 480, 402, 479, 511, 475].

Among the issues to be answered before such an approach becomes more rigorous, let us note: (1) what is the shape of the string landscape; (2) what constants should we scan. It is indeed important to distinguish the parameters that are actually fine-tuned in order to determine those that we should hope to explain in this way $[537,538]$. Here theoretical physics is indeed important since it should determine which of the numerical coincidences are coincidences and which are expected for some unification or symmetry reasons; (3) how is the landscape populated; (4) what is the measure to be used in order and what is the correct way to compute anthropically-conditioned probabilities.

While considered as not following the standard scientific approach, this is the only existing window on some understanding of the value of the fundamental constants. 


\section{Conclusions}

The study of fundamental constants has witnessed tremendous progresses in the past years. In a decade, the constraints on their possible space and time variations have flourished. They have reached higher precision and new systems, involving different combinations of constants and located at different redshifts, have been considered. This has improved our knowledge on the equivalence principle and allowed to test it on astrophysical and cosmological scales. We have reviewed them in Section 3 and Section 4. We have emphasized the experimental observational progresses expected in the coming years such as the E-ELT, radio observations, atomic clocks in space, or the use of gravitational waves.

From a theoretical point of view, we have described in Section 5 the high-energy models that predict such variation, as well as the link with the origin of the acceleration of the universe. In all these cases, a spacetime varying fundamental constant reflects the existence of an almost massless field that couples to matter. This will be at the origin of a violation of the universality of free fall and thus of utmost importance for our understanding of gravity and of the domain of validity of general relativity. Huge progress has been made in the understanding of the coupled variation of different constants. While more model-dependent, this allows one to set stronger constraints and eventually to open an observational window on unification mechanisms.

To finish, we have discussed in Section 7 the ideas that try to understand the value of the fundamental constant. While considered as borderline with respect to the standard physical approach, it reveals the necessity of considering a universe larger than our own, and called the multiverse. It will also give us a hint on our location in this structure in the sense that the anthropic principle limits the Copernican principle at the basis of most cosmological models. We have stressed the limitations of this approach and the ongoing debate on the possibility to make it predictive.

To conclude, the puzzle about the large numbers pointed out by Dirac has led to a better understanding of the fundamental constants and of their roles in the laws of physics. They are now part of the general tests of general relativity, as well as a breadcrumbs to understand the origin of the acceleration of the universe and to more speculative structures, such as a multiverse structure, and possibly a window on string theory. 


\section{Acknowledgments}

I would like to thank all my collaborators on this topic, Alain Coc, Pierre Descouvemont, Sylvia Ekström, George Ellis, Georges Meynet, Nelson Nunes, Keith Olive and Elisabeth Vangioni as well as Bénédicte Leclercq and Roland Lehoucq.

I also thank many colleagues for sharing their thoughts on the subject with me, first at the Institut d'Astrophysique de Paris, Luc Blanchet, Michel Cassé, Gilles Esposito-Farèse, Bernard Fort, Guillaume Faye, Jean-Pierre Lasota, Yannick Mellier, Patrick Petitjean; in France, Francis Bernardeau, Sébastien Bize, Françoise Combes, Thibault Damour, Nathalie Deruelle, Christophe Salomon, Carlo Schimd, Peter Wolfe; and to finish worldwide, John Barrow, Thomas Dent, Victor Flambaum, Bala Iyer, Lev Kofman, Paolo Molaro, David Mota, Michael Murphy, Jeff Murugan, Cyril Pitrou, Anan Srianand, Gabriele Veneziano, John Webb, Amanda Weltman, Christof Wetterich. To finish, I thank Clifford Will for motivating me to write this review.

This work was supported by a PEPS-PTI grant from CNRS (2009-2011) and the PNCG (2010) but, despite all our efforts, has not been supported by the French-ANR. 


\section{A Notations}

\section{A.1 Constants}

The notations and numerical values of the constants used in this review are summarized in Table 1 and Table 2 .

\section{A.2 Sensitivity coefficients}

The text introduces several sensitivity coefficients. We recall their definition here.

- Given an observable $O$, the value of which depends on a set of primary parameters $G_{k}$, the sensitivity of the measured value of $O$ to these parameters is

$$
\frac{\mathrm{d} \ln O}{\mathrm{~d} \ln G_{k}}=c_{k}
$$

The value of the quantities $c_{k}$ requires a physical description of the system.

- the parameters $G_{k}$ can be related to a set of fundamental constant $\alpha_{i}$ and we define

$$
\frac{\mathrm{d} \ln G_{k}}{\mathrm{~d} \ln \alpha_{i}}=d_{k i}
$$

The computation of the coefficients $d_{k i}$ requires one to specify the theoretical framework and depends heavily on our knowledge of nuclear physics and the assumptions on unification.

- A particular sets of parameters $d_{k i}$ has been singled out for the sensitivity of the mass of a body $A$ to a variation of the fundamental constants

$$
\frac{\mathrm{d} \ln m_{A}}{\mathrm{~d} \alpha_{i}}=f_{A i}
$$

One also introduces

$$
\frac{\mathrm{d} \ln m_{A}}{\mathrm{~d} \ln \alpha_{i}}=\lambda_{A i}
$$

so that

$$
\lambda_{A i}=\alpha_{i} f_{A i} .
$$

- In models where the variation of the fundamental constants are induced by the variation of a scalar field with define

$$
\frac{\mathrm{d} \ln \alpha_{i}}{\mathrm{~d} \phi}=s_{i}(\phi)
$$

- In class of models the variation of the constants can be related to the gravitational potential by

$$
\frac{\mathrm{d} \ln \alpha_{i}}{\mathrm{~d} \Phi_{N}}=k_{i}
$$




\section{A.3 Background cosmological spacetime}

We consider that the spacetime is describe by a manifold $\mathcal{M}$ with metric $g_{\mu \nu}$ with signature $(-,+,+,+)$. In the case of a Minkowsky spacetime $g_{\mu \nu}=\eta_{\mu \nu}$.

In the cosmological context, we will describe the universe by a Friedmann-Lemaître spacetime with metric

$$
\mathrm{d} s^{2}=-\mathrm{d} t^{2}+a^{2}(t) \gamma_{i j} \mathrm{~d} x^{i} \mathrm{~d} x^{j}
$$

where $t$ is the cosmic time, $a$ the scale factor and $\gamma_{i j}$ the metric on the constant time hypersurfaces. The Hubble function is defined as $H \equiv \dot{a} / a$. We also define the redshift by the relation $1+z=a_{0} / a$, with $a_{0}$ the scale factor evaluated today.

The evolution of the scale factor is dictated by the Friedmann equation

$$
H^{2}=\frac{8 \pi G}{3} \rho-\frac{K}{a^{2}}+\frac{\Lambda}{3}
$$

where $\rho={ }_{-} i \rho_{i}$ is the total energy density of the matter components in the universe. Assuming the species $i$ has a constant equation of state $w_{i}=P_{i} / \rho_{i}$, each component evolves as $\rho_{i}=\rho_{i 0}(1+$ $z)^{2\left(1+w_{i}\right)}$. The Friedmann equation can then be rewritten as

$$
\frac{H^{2}}{H_{0}^{2}}=\sum \Omega_{i}(1+z)^{3\left(1+w_{i}\right)}+\Omega_{K}(1+z)^{2}+\Omega_{\Lambda},
$$

with the density parameters defined by

$$
\Omega_{i} \equiv \frac{8 \pi G \rho_{i 0}}{3 H_{0}^{2}}, \quad \Omega_{i} \equiv-\frac{K}{3 H_{0}^{2}}, \quad \Omega_{\Lambda} \equiv \frac{\Lambda}{3 H_{0}^{2}} .
$$

They clearly satisfy $\sum \Omega_{i}+\Omega_{K}+\Omega_{\Lambda}=1$.

Concerning the properties of the cosmological spacetime, I follow the notations and results of [409]. 


\section{References}

[1] Accetta, F.S., Krauss, L.M. and Romanelli, P., "New limits on the variability of $G$ from big bang nucleosynthesis", Phys. Lett. B, 248, 146, (1990). (Cited on pages 83 and 84.)

[2] Acquaviva, V., Baccigalupi, C., Leach, S.M., Liddle, A.R. and Perrotta, F., "Structure formation constraints on the Jordan-Brans-Dicke theory", Phys. Rev. D, 71, 104025, (2005). [DOI], [astro-ph/0412052]. (Cited on page 83.)

[3] Adams, F.C., "Stars in other universes: stellar structure with different fundamental constants", J. Cosmol. Astropart. Phys., 2008(08), 010, (2008). [DOI], [arXiv:0807.3697 [astroph]]. (Cited on page 63.)

[4] Adelberger, E.G., "New tests of Einstein's equivalence principle and Newton's inverse-square law", Class. Quantum Grav., 18, 2397-2405, (2001). [DOI]. (Cited on page 19.)

[5] Agrawal, V., Barr, S.M., Donoghue, J.F. and Seckel, D., "Anthropic considerations in multiple-domain theories and the scale of electroweak symmetry breaking", Phys. Rev. Lett., 80, 1822, (1998). [DOI], [hep-ph/9801253]. (Cited on page 111.)

[6] Agrawal, V., Barr, S.M., Donoghue, J.F. and Seckel, D., "Viable range of the mass scale of the standard model", Phys. Rev. D, 57, 5480-5492, (1998). [DOI], [hep-ph/9707380]. (Cited on page 111.)

[7] Aguirre, A., "Making predictions in a multiverse: conundrums, dangers, coincidences", in Carr, B.J., ed., Universe or Multiverse?, pp. 367-386, (Cambridge University Press, Cambridge; New York, 2007). [astro-ph/0506519], [Google Books]. (Cited on page 113.)

[8] Amarilla, L. and Vucetich, H., "Brane-world cosmology and varying G", Int. J. Mod. Phys. A, 25, 3835-3856, (2010). [DOI], [0908.2949]. (Cited on page 101.)

[9] Amendola, L., Baldi, M. and Wetterich, C., "Quintessence cosmologies with a growing matter component", Phys. Rev. D, 78, 023015, (2008). [DOI], [arXiv:0706.3064 [astro-ph]]. (Cited on pages 101 and 102.)

[10] Anchordoqui, L., Barger, V., Goldberg, H. and Marfatia, D., "Phase transition in the fine structure constant", Phys. Lett. B, 660, 529, (2008). [arXiv:0711.4055 [hep-ph]]. (Cited on page 101.)

[11] Anchordoqui, L. and Goldberg, H., "Time variation of the fine structure constant driven by quintessence", Phys. Rev. D, 68, 083513, (2003). [DOI], [hep-ph/0306084]. (Cited on pages 24 and 102.)

[12] Anderson, J.D., Campbell, J.K., Jurgens, R.F. and Lau, E.L., "Recent Developments in Solar-System Tests of General Relativity", in Sato, H. and Nakamura, T., eds., The Sixth Marcel Grossmann Meeting: On recent developments in theoretical and experimental general relativity, gravitation and relativistic field theories, Proceedings of the meeting held at Kyoto International Conference Hall, Kyoto, Japan, 23-29 June 1991, pp. 353-355, (World Scientific, Singapore, 1992). (Cited on page 77.)

[13] Andreev, O.Y., Labzowsky, L.N., Plunien, G. and Soff, G., "Testing the time dependence of the fundamental constants in the spectra of multicharged ions", Phys. Rev. Lett., 94, 243002, (2005). [DOI], [physics/0505081]. (Cited on page 33.) 
[14] Angstmann, E.J., Dzuba, V.A. and Flambaum, V.V., "Atomic clocks and the search for variation of the fine structure constant", Phys. Rev. A, 70, 014102, (2004). [DOI], [physics/0407141]. (Cited on pages 45 and 105.)

[15] Angstmann, E.J., Dzuba, V.A., Flambaum, V.V., Nevsky, A.Y. and Karshenboim, S.G., "Narrow atomic transitions with enhanced sensitivity to variation of the fine structure constant", J. Phys. B: At. Mol. Opt. Phys., 39, 1937, (2006). [DOI], [physics/0511180]. (Cited on page 33.)

[16] Arai, K., Hashimoto, M. and Fukui, T., "Primordial nucleosynthesis in the Brans-Dicke theory with a variable cosmological term", Astron. Astrophys., 179, 17, (1987). [ADS]. (Cited on page 83.)

[17] Ashby, N., Heavner, T.P., Jefferts, S.R., Parker, T.E., Radnaev, A.G. and Dudin, Y.O., "Testing Local Position Invariance with Four Cesium-Fountain Primary Frequency Standards and Four NIST Hydrogen Masers", Phys. Rev. Lett., 98, 070802, (2007). [DOI]. (Cited on page 105.)

[18] Ashenfelter, T., Mathews, G.J. and Olive, K.A., "The chemical evolution of Mg isotopes vs. the time variation of the fine structure constant", Phys. Rev. Lett., 92, 041102, (2004). [DOI], [astro-ph/0309197]. (Cited on page 49.)

[19] Audi, G., "The history of nuclidic masses and of their evaluation", Int. J. Mass Spectrom., 251, 85-94, (2006). [DOI], [physics/0602050]. (Cited on page 74.)

[20] Avelino, P.P., Martins, C.J.A.P., Nunes, N.J. and Olive, K.A., "Reconstructing the dark energy equation of state with varying constant", Phys. Rev. D, 74, 083508, (2006). [DOI], [astro-ph/0605690]. (Cited on pages 25 and 102.)

[21] Avelino, P.P., Martins, C.J.A.P. and Rocha, G., "Looking for a varying $\alpha$ in the cosmic microwave background", Phys. Rev. D, 62, 123508, (2000). [DOI], [astro-ph/0008446]. (Cited on page 65.)

[22] Avelino, P.P. et al., "Early-universe constraints on a time-varying fine structure constant", Phys. Rev. D, 64, 103505, (2001). [DOI], [astro-ph/0102144]. (Cited on pages 65 and 67.)

[23] Baeßler, S., Heckel, B.R., Adelberger, E.G., Gundlach, J.H., Schmidt, U. and Swanson, H.E., "Improved Test of the Equivalence Principle for Gravitational Self-Energy", Phys. Rev. Lett., 83, 3585-3588, (1999). [DOI]. (Cited on page 19.)

[24] Bahcall, J.N., Steinhardt, C.L. and Schlegel, D., "Does the fine-structure constant vary with cosmological epoch?", Astrophys. J., 600, 520, (2004). [DOI], [astro-ph/0301507]. (Cited on page 58.)

[25] Bambi, C. and Drago, A., "Constraints on temporal variation of fundamental constants from GRBs", Astropart. Phys., 29, 223, (2008). [DOI], [arXiv:0711.3569 [hep-ph]]. (Cited on page 82.)

[26] Barrow, J.D., "A cosmological limit on the possible variation of G", Mon. Not. R. Astron. Soc., 184, 677, (1978). (Cited on pages 83 and 84.)

[27] Barrow, J.D., "Natural Units Before Planck", Quart. J. R. Astron. Soc., 24, 24-26, (1983). [ADS]. (Cited on page 15.)

[28] Barrow, J.D., "Observational limits on the time evolution of extra spatial dimensions", Phys. Rev. D, 35, 1805, (1987). [DOI]. (Cited on page 89.) 
[29] Barrow, J.D., The Constants of Nature: From Alpha to Omega - The Numbers that Encode the Deepest Secrets of the Universe, (Jonathan Cape, London, 2002). (Cited on pages 9 and 14.)

[30] Barrow, J.D., "Cosmological bounds on spatial variations of physical constants", Phys. Rev. $D, \mathbf{7 1}, 083520,(2005)$. [DOI], [astro-ph/0503434]. (Cited on page 106.)

[31] Barrow, J.D., "Varying constants", Philos. Trans. R. Soc. London, Ser. A, 363, 2139, (2005). [astro-ph/0511440]. (Cited on page 8.)

[32] Barrow, J.D. and Li, B., "Varying-alpha cosmologies with potentials", Phys. Rev. D, 78, 083536, (2008). [DOI], [arXiv:0808.1580 [gr-qc]]. (Cited on page 100.)

[33] Barrow, J.D. and Magueijo, J., "Can a changing $\alpha$ explain the Supernovae results?", Astrophys. J., 532, L87, (2000). [DOI], [astro-ph/9907354]. (Cited on page 24.)

[34] Barrow, J.D. and Shaw, D.J., "Varying-alpha: new constraints from seasonal variations", Phys. Rev. D, 78, 067304, (2008). [DOI], [arXiv:0806.4317 [hep-ph]]. (Cited on page 105.)

[35] Barrow, J.D. and Tipler, F.J., The Anthropic Cosmological Principle, (Oxford University Press, Oxford; New York, 1986). [Google Books]. (Cited on page 112.)

[36] Battye, R.A., Crittenden, R. and Weller, J., "Cosmic concordance and the fine structure constant", Phys. Rev. D, 63, 043505, (2001). [DOI], [astro-ph/0008265]. (Cited on page 65.)

[37] Bauch, A. and Weyers, S., "New experimental limit on the validity of local position invariance", Phys. Rev. D, 65, 081101R, (2002). [DOI]. (Cited on page 104.)

[38] Beane, S.R. and Savage, M.J., "Variation of fundamental couplings and nuclear forces", Nucl. Phys. A, 717, 91, (2003). [DOI], [hep-ph/0206113]. (Cited on pages 74 and 95.)

[39] Bekenstein, J.D., "Fine-structure constant: Is it really a constant", Phys. Rev. D, 25, 1527, (1982). [DOI]. (Cited on pages 100, 101, and 108.)

[40] Bekenstein, J.D., "Fine-structure constant variability, equivalence principle and cosmology", Phys. Rev. D, 66, 123514, (2002). [DOI]. (Cited on page 100.)

[41] Bekenstein, J.D. and Schiffer, M., "Varying-fine structure 'constant' and charged black-hole", Phys. Rev. D, 80, 123508, (2009). [DOI], [arXiv:0906.4557 [gr-qc]]. (Cited on page 101.)

[42] Beloy, K., Borschevsky, A., Schwerdtfeger, P. and Flambaum, V.V., "Enhanced Sensitivity to the Time Variation of the Fine-Structure Constant and $m_{p} / m_{e}$ in Diatomic Molecules: A Closer Examination of Silicon Monobromide", Phys. Rev. A, 82, 022106, (2010). [DOI], [arXiv:1007.0393 [physics.atom-ph]]. (Cited on page 33.)

[43] Benvenuto, O.G., García-Berro, E. and Isern, J., "Asteroseismology bound on $\dot{G} / G$ from pulsating white dwarfs", Phys. Rev. D, 69, 082002, (2004). [DOI]. (Cited on page 81.)

[44] Berengut, J.C., Dzuba, V.A. and Flambaum, V.V., "Enhanced Laboratory Sensitivity to Variation of the Fine-Structure Constant using Highly Charged Ions", Phys. Rev. Lett., 105, 120801, (2010). [DOI], [arXiv:1007.1068 [physics.atom-ph]]. (Cited on page 33.)

[45] Berengut, J.C., Dzuba, V.A., Flambaum, V.V., Kozlov, M.G., Marchenko, M.V., Murphy, M.T. and Webb, J.K., "Laboratory spectroscopy and the search for space-time variation of the fine structure constant using QSO spectra", arXiv, e-print, (2006). [arXiv:physics/0408017]. (Cited on page 45.) 
[46] Berengut, J.C., Dzuba, V.A., Flambaum, V.V. and Porsev, S.G., "A proposed experimental method to determine $\alpha$-sensitivity of splitting between ground and $7.6 \mathrm{eV}$ isomeric states in ${ }^{229} \mathrm{Th} "$, Phys. Rev. Lett., 102, 210801, (2009). [DOI], [arXiv:0903.1891 [physics.atom-ph]]. (Cited on page 34.)

[47] Berengut, J.C. and Flambaum, V.V., "Astronomical and laboratory searches for spacetime variation of fundamental constants", J. Phys.: Conf. Ser., 264, 012010, (2010). [DOI], [arXiv:1009.3693 [physics.atom-ph]]. (Cited on page 8.)

[48] Berengut, J.C. and Flambaum, V.V., "Manifestations of a spatial variation of fundamental constants on atomic clocks, Oklo, meteorites, and cosmological phenomena", arXiv, e-print, (2010). [arXiv:1008.3957 [physics.atom-ph]]. (Cited on page 51.)

[49] Berengut, J.C., Flambaum, V.V. and Dmitriev, V.F., "Effect of quark-mass variation on big bang nucleosynthesis", Phys. Lett. B, 683, 114, (2010). [arXiv:0907.2288 [nucl-th]]. (Cited on page 73.)

[50] Berengut, J.C., Flambaum, V.V., King, J.A., Curran, S.J. and Webb, J.K., "Is there further evidence for spatial variation of fundamental constants?", arXiv, e-print, (2010). [arXiv:1009.0591 [astro-ph.CO]]. (Cited on pages 51 and 106.)

[51] Bergström, L., Iguri, S. and Rubinstein, H., "Constraints on the variation of the fine structure constant from big bang nucleosynthesis", Phys. Rev. D, 60, 045005, (1999). [DOI], [astroph/9902157]. (Cited on page 70.)

[52] Bertolami, O., Lehnert, R., Potting, R. and Ribeiro, A., "Cosmological acceleration, varying couplings, and Lorentz breaking", Phys. Rev. D, 69, 083513, (2004). [DOI], [arXiv:astroph/0310344]. (Cited on page 22.)

[53] Bertotti, B., Iess, L. and Tortora, P., "A test of general relativity using radio links with the Cassini spacecraft", Nature, 425, 374-376, (2003). [DOI]. (Cited on page 20.)

[54] Biesiada, M. and Malec, B., "A new white dwarf constraint on the rate of change of the gravitational constant", Mon. Not. R. Astron. Soc., 350, 644, (2004). [DOI], [astro-ph/0303489]. (Cited on page 81.)

[55] BIPM, The International System of Units (SI), (BIPM, Sèvres, 2006), 8th edition. Online version (accessed 1 March 2011):

http://www.bipm.org/en/si/si_brochure/. (Cited on page 15.)

[56] Birge, R.T., "Probable Values of the General Physical Constants", Rev. Mod. Phys., 1, 1, (1929). (Cited on page 13.)

[57] Bize, S. et al., "Testing the Stability of Fundamental Constants with ${ }^{199} \mathrm{Hg}^{+}$Single-Ion Optical Clock", Phys. Rev. Lett., 90, 150802, (2003). [DOI], [physics/0212109]. (Cited on pages 29 and 30.)

[58] Bize, S. et al., "Cold atom clocks and applications", J. Phys. B: At. Mol. Opt. Phys., 38, S449-S468, (2005). [DOI], [physics/0502117]. (Cited on page 29.)

[59] Bjorken, J.D., "Standard Model Parameters and the Cosmological Constant", Phys. Rev. D, 64, 085008, (2001). [DOI], [hep-ph/0103349]. (Cited on page 9.) 
[60] Blanchet, L., "Gravitational Radiation from Post-Newtonian Sources and Inspiralling Compact Binaries", Living Rev. Relativity, 9, lrr-2006-4, (2006). [gr-qc/0202016]. URL (accessed 27 September 2010):

http://www.livingreviews.org/lrr-2006-4. (Cited on page 20.)

[61] Blatt, S. et al., "New Limits on Coupling of Fundamental Constants to Gravity Using ${ }^{87}$ Sr Optical Lattice Clocks", Phys. Rev. Lett., 100, 140801, (2008). [DOI], [arXiv:0801.1874 [physics.atom-ph]]. (Cited on pages 29, 31, and 105.)

[62] Bohlin, R., Jenkins, E.B., Spitzer Jr, L., York, D.G., Hill, J.K., Savage, B.D. and Snow Jr, T.P., "A survey of ultraviolet interstellar absorption lines", Astrophys. J. Suppl. Ser., 51, 277-308, (1983). [DOI]. (Cited on page 60.)

[63] Bonifacio, P. et al., "First stars VII - Lithium in extremely metal poor dwarfs", Astron. Astrophys., 462, 851-864, (2007). [DOI], [astro-ph/0610245]. (Cited on page 69.)

[64] Bostrom, N., Anthropic Bias: Observation Selection Effects in Science and Philosophy, (Routledge, New York; London, 2002). [Google Books]. (Cited on pages 110 and 113.)

[65] Bousso, R., Hall, L.J. and Nomura, Y., "Multiverse understanding of cosmological coincidences", Phys. Rev. D, 80, 063510, (2009). [DOI], [arXiv:0902.2263 [hep-th]]. (Cited on page 112.)

[66] Bousso, R. and Polchinski, J., "Quantization of Four-form Fluxes and Dynamical Neutralization of the Cosmological Constant", J. High Energy Phys., 2000(06), 006, (2000). [DOI], [hep-th/0004134]. (Cited on page 110.)

[67] Brans, C. and Dicke, R.H., "Mach's Principle and a Relativistic Theory of Gravitation", Phys. Rev., 124, 925-935, (1961). [DOI]. (Cited on pages 7 and 85.)

[68] Brax, P. and Martin, J., "Dark Energy and the MSSM", Phys. Rev. D, 75, 083507, (2007). [DOI], [hep-th/0605228]. (Cited on page 109.)

[69] Brax, P. and Martin, J., "Moduli Fields as Quintessence and the Chameleon", Phys. Lett. $B, \mathbf{6 4 7}, 320,(2007)$. [hep-th/0612208]. (Cited on page 94.)

[70] Brax, P., van de Bruck, C., Davis, A.-C., Khoury, J. and Weltman, A., "Detecting dark energy in orbit: The cosmological chameleon", Phys. Rev. D, 70, 123518, (2004). [DOI], [astro-ph/0408415]. (Cited on page 99.)

[71] Brax, P., van de Bruck, C., Mota, D.F., Nunes, N.J. and Winther, H.A., "Chameleons with field-dependent couplings", Phys. Rev. D, 82, (2010). [DOI], [arXiv:1006.2796 [astro-ph.CO]]. (Cited on page 99.)

[72] Bronnikov, K.A. and Kononogov, S.A., "Possible variations of the fine structure constant $\alpha$ and their metrological significance", Metrologia, 43, R1, (2006). [DOI], [gr-qc/0604002]. (Cited on page 8.)

[73] Byrne, M. and Kolda, C., "Quintessence and varying $\alpha$ from shape moduli", arxiv, e-print, (2004). [arxiv:hep-ph/0402075]. (Cited on page 101.)

[74] Calmet, X. and Fritzsch, H., "The Cosmological Evolution of the Nucleon Mass and the Electroweak Coupling Constants", Eur. Phys. J. C, 24, 639-642, (2002). [DOI], [hep-ph/0112110]. (Cited on page 93.) 
[75] Calmet, X. and Fritzsch, H., "Symmetry Breaking and Time Variation of Gauge Couplings", Phys. Lett. B, 540, 173, (2002). [hep-ph/0204258]. (Cited on page 93.)

[76] Calmet, X. and Fritzsch, H., "A time variation of proton-electron mass ratio and grand unification", Europhys. Lett., 76, 1064, (2006). [DOI], [astro-ph/0605232]. (Cited on page 93.)

[77] Campbell, B.A. and Olive, K.A., "Nucleosynthesis and the time dependence of fundamental couplings", Phys. Lett. B, 345, 429-434, (1995). [hep-ph/9411272]. (Cited on pages 70, 75, and 93.)

[78] Carilli, C.L. et al., "Astronomical Constraints on the Cosmic Evolution of the Fine Structure Constant and Possible Quantum Dimensions", Phys. Rev. Lett., 85, 5511-5514, (2000). [DOI]. (Cited on page 53.)

[79] Carr, B.J., ed., Universe or Multiverse?, (Cambridge University Press, Cambridge; New York, 2007). [Google Books]. (Cited on page 110.)

[80] Carr, B.J. and Rees, M.J., "The anthropic principle and the structure of the physical world", Nature, 278, 605-612, (1979). [DOI]. (Cited on pages 7 and 111.)

[81] Carroll, S.M., "Quintessence and the Rest of the World: Suppressing Long-Range Interactions", Phys. Rev. Lett., 81, 3067-3070, (1998). [DOI]. (Cited on pages 23 and 24.)

[82] Carter, B., "Large number coincidences and the anthropic principle in cosmology", in Longair, M.S., ed., Confrontation of Cosmological Theories with Observational Data, Proceedings of the 63rd Symposium of the International Astronomical Union (Copernicus Symposium II), held in Cracow, Poland, 10-12 September, 1973, pp. 291-298, (Reidel, Dordrecht, 1974). [ADS]. (Cited on pages 7, 110, and 112.)

[83] Carter, B., "The anthropic principle and its implication for biological evolution", Philos. Trans. R. Soc. London, Ser. A, 310, 347, (1983). [DOI]. (Cited on page 7.)

[84] Casas, J.A., García-Bellido, J. and Quirós, M., "Nucleosynthesis Bounds On Jordan-BransDicke Theories Of Gravity", Mod. Phys. Lett. A, 7, 447, (1992). [DOI]. (Cited on page 83.)

[85] Cembranos, J.A.R., Olive, K.A., Peloso, M. and Uzan, J.-P., "Quantum corrections to the cosmological evolution of conformally coupled fields", J. Cosmol. Astropart. Phys., 2009(07), 025, (2009). [DOI], [arXiv:0905.1989 [astro-ph.CO]]. (Cited on page 87.)

[86] Centurión, M., Molaro, P. and Levshakov, S., "Calibration issues in $\Delta \alpha \alpha "$, Mem. Soc. Astron. Ital., 80, 929, (2009). (Cited on page 50.)

[87] Chacko, Z., Grojean, C. and Perelstein, M., "Fine structure constant variation from a late phase transition", Phys. Lett. B, 565, 169, (2003). [hep-ph/0204142]. (Cited on page 101.)

[88] Chamoun, N., Landau, S.J., Mosquera, M.E. and Vucetich, H., "Helium and deuterium abundances as a test for the time variation of the baryonic density, fine structure constant and the Higgs vacuum expectation value", J. Phys. G: Nucl. Part. Phys., 34, 163, (2007). [DOI], [astro-ph/0508378]. (Cited on page 74.)

[89] Chan, K.C. and Chu, M.-C., "Constraining the variation of $G$ by cosmic microwave background anisotropies", Phys. Rev. D, 75, 083521, (2007). [DOI], [astro-ph/0611851]. (Cited on page 83.) 
[90] Chand, H., Petitjean, P., Srianand, R. and Aracil, B., "Probing the cosmological variation of the fine-structure constant: Results based on VLT-UVES sample", Astron. Astrophys., 417, 853, (2004). [DOI], [astro-ph/0401094]. (Cited on pages 49, 50, and 59.)

[91] Chand, H., Petitjean, P., Srianand, R. and Aracil, B., "Probing the time-variation of the fine-structure constant: Results based on Si IV doublets from a UVES sample", Astron. Astrophys., 430, 47-58, (2005). [DOI], [astro-ph/0408200]. (Cited on pages 46, 47, and 59.)

[92] Chand, H., Petitjean, P., Srianand, R. and Aracil, B., "On the variation of the fine-structure constant: Very high resolution spectrum of QSO HE 0515-4414", Astron. Astrophys., 451, 45, (2006). [DOI], [astro-ph/0601194]. (Cited on page 51.)

[93] Chandler, J.F., Reasenberg, R.D. and Shapiro, I.I., "New bounds on $\dot{G}$ ", Bull. Am. Astron. Soc., 25, 1233, (1993). (Cited on page 77.)

[94] Chen, X. and Kamionkowski, M., "Cosmic microwave background temperature and polarization anisotropy in Brans-Dicke cosmology", Phys. Rev. D, 60, 104036, (1999). [DOI]. (Cited on page 83.)

[95] Chengalur, J.N. and Kanekar, N., "Constraining the variation of fundamental constants using 18 cm OH lines", Phys. Rev. Lett., 91, 241302, (2003). [DOI], [astro-ph/0310764]. (Cited on pages 53, 54, and 59.)

[96] Chiba, T. and Khori, K., "Quintessence cosmology and varying $\alpha$ ", Prog. Theor. Phys., 107, 631, (2002). [DOI], [hep-ph/0111086]. (Cited on pages 24, 102, and 108.)

[97] Chiba, T., Kobayashi, T., Yamaguchi, M. and Yokoyama, J., "Time variation of protonelectron mass ratio and fine structure constant with runaway dilaton", Phys. Rev. D, 75, 043516, (2007). [DOI], [hep-ph/0610027]. (Cited on page 99.)

[98] Chin, C. and Flambaum, V.V., "Enhancement of variation of fundamental constants in ultracold atom and molecule systems near Feshbach resonances", Phys. Rev. Lett., 96, 230801, (2006). [DOI], [cond-mat/0603607]. (Cited on page 33.)

[99] Chupp, T.E., Hoare, R.J., Loveman, R.A., Oteiza, E.R., Richardson, J.M., Wagshul, M.E. and Thompson, A.K., "Results of a new test of local Lorentz invariance: A search for mass anisotropy in ${ }^{21} \mathrm{Ne} "$, Phys. Rev. Lett., 63, 1541-1545, (1989). [DOI]. (Cited on page 18.)

[100] Cingöz, A., Lapierre, A., Nguyen, A.-T., Leefer, N., Budker, D., Lamoreaux, S.K. and Torgerson, J.R., "Limit on the Temporal Variation of the Fine-Structure Constant Using Atomic Dysprosium", Phys. Rev. Lett., 98, 040801, (2008). [DOI], [physics/0609014]. (Cited on pages 29, 31, and 104.)

[101] Civitarese, O., Moliné, M.A. and Mosquera, M.E., "Cosmological bounds to the variation of the Higgs vacuum expectation value: BBN constraints", Nucl. Phys. A, 846, 157, (2010). [DOI]. (Cited on page 75.)

[102] Clifton, T., Barrow, J.D. and Scherrer, R.J., "Constraints on the variation of $G$ from primordial nucleosynthesis", Phys. Rev. D, 71, 123526, (2005). [DOI]. (Cited on page 83.)

[103] Coc, A., Ekström, S., Descouvemont, P., Meynet, G., Olive, K.A., Uzan, J.-P. and Vangioni, E., "Constraints on the variations of fundamental couplings by stellar models", Mem. Soc. Astron. Ital., 80, 809-813, (2009). [ADS]. (Cited on pages 61 and 63.) 
[104] Coc, A., Nunes, N.J., Olive, K.A., Uzan, J.-P. and Vangioni, E., "Coupled variations of the fundamental couplings and primordial nucleosynthesis", Phys. Rev. D, 76, 023511, (2007). [DOI], [astro-ph/0610733]. (Cited on pages 63, 71, 75, 93, and 94.)

[105] Coc, A., Olive, K.A., Uzan, J.-P. and Vangioni, E., "Big bang nucleosynthesis constraints on scalar-tensor theories of gravity", Phys. Rev. D, 73, 083525, (2006). [DOI], [astro-ph/0601299]. (Cited on pages $72,75,83,84$, and 87.)

[106] Coc, A., Olive, K., Uzan, J.-P. and Vangioni, E., "Non-universal scalar-tensor theories and big bang nucleosynthesis", Phys. Rev. D, 79, 103512, (2009). [DOI]. (Cited on pages 83 and 87.)

[107] Coc, A. and Vangioni, E., "Big-Bang Nucleosynthesis with updated nuclear data", J. Phys.: Conf. Ser., 202, 012001, (2010). [DOI]. (Cited on pages 69 and 72.)

[108] Coc, A., Vangioni-Flam, E., Descouvemont, P., Adahchour, A. and Angulo, C., "Updated big bang nucleosynthesis compared with Wilkinson Microwave Anisotropy Probe observations and the abundance of light elements", Astrophys. J., 600, 544, (2004). [DOI], [astroph/0309480]. (Cited on pages 69 and 87.)

[109] Combes, F., "Radio measurements of constant variation, and perspective with ALMA", Mem. Soc. Astron. Ital., 80, 888, (2009). (Cited on page 46.)

[110] Cook, A.H., "Secular changes of the units and constant of physics", Nature, 180, 1194, (1957). [DOI]. (Cited on page 17.)

[111] Cook, C.W., Fowler, W.A., Lauritsen, C.C. and Lauritsen, T., "B ${ }^{12}, \mathrm{C}^{12}$, and the Red Giants", Phys. Rev. D, 107, 508, (1957). [DOI]. (Cited on page 61.)

[112] Copeland, E.J., Nunes, N.J. and Pospelov, M., "Models of quintessence coupled to the electromagnetic field and the cosmological evolution of $\alpha "$, Phys. Rev. D, 69, 023501, (2004). [DOI], [hep-ph/0307299]. (Cited on pages 24 and 102.)

[113] Copi, C.J., Davis, A.N. and Krauss, L.M., "New Nucleosynthesis Constraint on the Variation of G", Phys. Rev. Lett., 92, 171301, (2004). [DOI]. (Cited on page 83.)

[114] Cremmer, E. and Scherk, J., "Spontaneous Compactification of Extra Space Dimensions", Nucl. Phys. B, 118, 61, (1977). [DOI]. (Cited on page 89.)

[115] Cristiani, S. et al., "The CODEX-ESPRESSO experiment: cosmic dynamics, fundamental physics, planets and much more...", Nuovo Cimento B, 122, 1165-1170, (2007). [DOI], [arXiv:0712.4152 [astro-ph]]. (Cited on page 60.)

[116] Cyburt, R.H., Fields, B.D. and Olive, K.A., "An update on the big bang nucleosynthesis prediction for ${ }^{7} \mathrm{Li}$ : the problem worsens", J. Cosmol. Astropart. Phys., 2008(11), 012, (2008). [DOI], [arXiv:0808.2818 [astro-ph]]. (Cited on page 70.)

[117] Cyburt, R.H., Fields, B.D., Olive, K.A. and Skillman, E., "New BBN limits on physics beyond the standard model from ${ }^{4} \mathrm{He} "$, Astropart. Phys., 23, 313-323, (2005). [DOI], [astroph/0408033]. (Cited on pages 68 and 84.)

[118] Damour, T., "Testing the equivalence principle: why and how?", Class. Quantum Grav., 13, A33-A41, (1996). [DOI], [gr-qc/9606080]. (Cited on pages 21 and 107.)

[119] Damour, T., "The Equivalence Principle and the Constants of Nature", Space Sci. Rev., 148, 191, (2009). [DOI], [arXiv:0906.3174 [gr-qc]]. (Cited on page 8.) 
[120] Damour, T. and Donoghue, J.F., "Constraints on the variability of quark masses from nuclear binding", Phys. Rev. D, 78, 014014, (2008). [DOI], [arXiv:0712.2968 [hep-ph]]. (Cited on pages $21,22,95$, and 111.)

[121] Damour, T. and Donoghue, J.F., "Equivalence Principle Violations and Couplings of a Light Dilaton", Phys. Rev. D, 82, 084033, 1-20, (2010). [arXiv:1007.2792 [gr-qc]]. (Cited on pages 99 and 107.)

[122] Damour, T. and Donoghue, J.F., "Phenomenology of the Equivalence Principle with Light Scalars", Class. Quantum Grav., 27, 202001, (2010). [DOI], [arXiv:1007.2790 [gr-qc]]. (Cited on pages $21,22,99$, and 107.)

[123] Damour, T. and Dyson, F.J., "The Oklo bound on the time variation of the fine-structure constant revisited", Nucl. Phys. B, 480, 37-54, (1996). [DOI], [hep-ph/9606486]. (Cited on pages 37,38 , and 39.)

[124] Damour, T. and Esposito-Farèse, G., "Tensor-multi-scalar theories of gravitation", Class. Quantum Grav., 9, 2093-2176, (1992). [DOI]. (Cited on pages 7, 85, 103, and 104.)

[125] Damour, T. and Esposito-Farèse, G., "Gravitational-wave versus binary-pulsar tests of strong-field gravity", Phys. Rev. D, 58, 042001, (1998). [DOI]. (Cited on pages 20 and 87.)

[126] Damour, T., Gibbons, G.W. and Gundlach, C., "Dark matter, time-varying G, and a dilaton field", Phys. Rev. Lett., 64, 123, (1990). [DOI]. (Cited on page 100.)

[127] Damour, T., Gibbons, G.W. and Taylor, J.H., "Limits on the Variability of G Using BinaryPulsar Data", Phys. Rev. Lett., 61, 1151-1154, (1988). [DOI], [ADS]. (Cited on page 78.)

[128] Damour, T. and Gundlach, C., "Nucleosynthesis constraints on an extended Jordan-BransDicke theory", Phys. Rev. D, 43, 3873, (1991). [DOI]. (Cited on page 83.)

[129] Damour, T. and Lilley, M., "String theory, gravity and experiment", in Bachas, C., Baulieu, L., Douglas, M., Kiritsis, E., Rabinovici, E., Vanhove, P., Windey, P. and Cugliandolo, L.F., eds., String Theory and the Real World: From Particle Physics to Astrophysics, Proceedings of the Les Houches Summer School, Session LXXXVII, 2 July - 27 July 2007, Les Houches Summer School Proceedings, 87, pp. 371-448, (Elsevier, Amsterdam, 2008). (Cited on pages 20,95, and 97.)

[130] Damour, T. and Nordtvedt, K., "General relativity as a cosmological attractor of tensorscalar theories", Phys. Rev. Lett., 70, 2217-2219, (1993). [DOI]. (Cited on page 87.)

[131] Damour, T. and Nordtvedt, K., "Tensor-scalar cosmological models and their relaxation toward general relativity", Phys. Rev. D, 48, 3436-3450, (1993). [DOI]. (Cited on page 87.)

[132] Damour, T., Piazza, F. and Veneziano, G., "Runaway dilaton and equivalence principle violations", Phys. Rev. Lett., 89, 081601, (2002). [DOI], [gr-qc/0204094]. (Cited on page 24.)

[133] Damour, T., Piazza, F. and Veneziano, G., "Violations of the equivalence principle in a dilaton-runaway scenario", Phys. Rev. D, 66, 046007, (2002). [DOI], [hep-th/0205111]. (Cited on pages 24 and 99.)

[134] Damour, T. and Pichon, B., "Big bang nucleosynthesis and tensor-scalar gravity", Phys. Rev. $D, \mathbf{5 9}, 123502,(1999)$. [DOI], [astro-ph/9807176]. (Cited on pages 24, 75, 83, 84, and 87.) 
[135] Damour, T. and Polyakov, A.M., "The string dilaton and a least coupling principle", Nucl. Phys. B, 423, 532-558, (1994). [DOI], [hep-th/9401069]. (Cited on pages 22, 88, 93, 97, 98, and 108.)

[136] Damour, T. and Polyakov, A.M., "String theory and gravity", Gen. Relativ. Gravit., 26, 1171, (1994). [DOI], [gr-qc/9411069]. (Cited on pages 88, 93, 97, and 108.)

[137] Damour, T. and Taylor, J.H., "On the Orbital Period Change of the Binary Pulsar PSR 1913+16", Astrophys. J., 366, 501-511, (1991). [DOI], [ADS]. (Cited on page 78.)

[138] Darling, J., "A laboratory for constraining cosmic evolution of the fine-structure constant: conjugate 18 centimeter OH lines toward PKS 1413+135 at $z=0.2467 "$ ", Astrophys. J., 612, 58, (2004). [DOI], [astro-ph/0405240]. (Cited on pages 54 and 59.)

[139] Davies, P.C.W., Davis, T.M. and Lineweaver, C.H., "Cosmology: Black holes constrain varying constants", Nature, 418, 602, (2002). [DOI]. (Cited on page 101.)

[140] Del'Innocenti, S. etal, "Time variation of Newton's constant and the age of globular clusters", Astron. Astrophys., 312, 345, (1996). (Cited on pages 79 and 80.)

[141] Demarque, P., Krauss, L.M., Guenther, D.B. and Nydam, D., "The Sun as a probe of varying G", Astrophys. J., 437, 870, (1994). [DOI]. (Cited on page 80.)

[142] Dent, T., "Varying alpha, thresholds and fermion masses", Nucl. Phys. B, 677, 471-484, (2004). [DOI], [hep-ph/0305026]. (Cited on page 94.)

[143] Dent, T., "Composition-dependent long range forces from varying $m_{p} / m_{e}$ ", J. Cosmol. Astropart. Phys., 2007(01), 013, (2007). [DOI], [hep-ph/0608067]. (Cited on pages 21 and 108.)

[144] Dent, T., "Eötvös bounds on couplings of fundamental parameters to gravity", Phys. Rev. Lett., 101, 041102, (2008). [DOI], [arXiv:0805.0318 [hep-ph]]. (Cited on page 109.)

[145] Dent, T. and Fairbairn, M., "Time varying coupling strength, nuclear forces and unification", Nucl. Phys. B, 653, 256, (2003). [DOI], [hep-ph/0112279]. (Cited on pages 95 and 111.)

[146] Dent, T., Stern, S. and Wetterich, C., "Primordial nucleosynthesis as a probe of fundamental physics parameters", Phys. Rev. D, 76, 063513, (2007). [DOI], [arXiv:0705.0696 [astro-ph]]. (Cited on pages 73,75 , and 94.)

[147] Dent, T., Stern, S. and Wetterich, C., "Unifying cosmological and recent time variations of fundamental couplings", Phys. Rev. D, 78, 103518, (2008). [DOI], [arXiv:0808.0702 [hep-ph]]. (Cited on pages 43,52 , and 94.)

[148] Dent, T., Stern, S. and Wetterich, C., "Competing bounds on the present-day time variation of fundamental constants", Phys. Rev. D, 79, 083533, (2009). [DOI], [arXiv:0812.4130 [hep-ph]]. (Cited on page 109.)

[149] Dent, T., Stern, S. and Wetterich, C., "Time variation of fundamental couplings and dynamical dark energy", J. Cosmol. Astropart. Phys., 2009(01), 038, (2009). [DOI], [arXiv:0809.4628 [hep-ph]]. (Cited on pages 94 and 108.)

[150] Dicke, R.H., "Dirac's Cosmology and the Dating of Meteorites", Nature, 183, 170-171, (1959). [DOI]. (Cited on page 42.)

[151] Dicke, R.H., "Dirac's Cosmology and Mach's Principle", Nature, 192, 440, (1961). [DOI]. (Cited on pages 7 and 110.) 
[152] Dicke, R.H., "Experimental relativity", in DeWitt, C.M. and DeWitt, B.S., eds., Relativity, Groups and Topology. Relativité, Groupes et Topologie, Lectures delivered at Les Houches during the 1963 session of the Summer School of Theoretical Physics, University of Grenoble, pp. 165-313, (Gordon and Breach, New York; London, 1964). (Cited on pages 21 and 100.)

[153] Dine, M., Nir, Y., Raz, G. and Volansky, T., "Time Variations in the Scale of Grand Unification", Phys. Rev. D, 67, 015009, (2003). [DOI], [hep-ph/0209134]. (Cited on page 94.)

[154] Dinh, T.H., Dunning, A., Dzuba, V.A. and Flambaum, V.V., "The sensitivity of hyperfine structure to nuclear radius and quark mass variation", Phys. Rev. A, 79, 054102, (2009). [DOI], [arXiv:0903.2090 [physics.atom-ph]]. (Cited on page 34.)

[155] Dirac, P.A.M., "The cosmological constants", Nature, 139, 323, (1937). [DOI]. (Cited on pages 7,76 , and 110.)

[156] Dirac, P.A.M., "A new basis for cosmology", Proc. R. Soc. London, Ser. A, 165, 199-208, (1938). [ADS]. (Cited on page 7.)

[157] Dmitriev, V.F. and Flambaum, V.V., "Limits on cosmological variation of quark masses and strong interaction", Phys. Rev. D, 67, 063513, (2003). [DOI], [astro-ph/0209409]. (Cited on pages 22 and 71.$)$

[158] Dmitriev, V.F., Flambaum, V.V. and Webb, J.K., "Cosmological varation of deuteron binding energy, strong interaction and quark masses from big bang nucleosynthesis", Phys. Rev. D, 69, 063506, (2004). [DOI], [astro-ph/0310892]. (Cited on page 71.)

[159] Donoghue, J.F., "The nuclear central force in the chiral limit", Phys. Rev. C, 74, 024002, (2006). [DOI], [nucl-th/0603016]. (Cited on pages 22, 95, and 96.)

[160] Donoghue, J.F., Dutta, K. and Ross, A., "Quark and lepton masses and mixing in the landscape", Phys. Rev. D, 73, 113002, (2006). [DOI], [hep-ph/0511219]. (Cited on page 111.)

[161] Donoghue, J.F., Dutta, K., Ross, A. and Tegmark, M., "Likely values of the Higgs vev", Phys. Rev. D, 81, 073003, (2010). [DOI], [arXiv:0903.1024 [hep-ph]]. (Cited on page 111.)

[162] Doran, M., "Can we test Dark Energy with Running Fundamental Constants?", J. Cosmol. Astropart. Phys., 2005(04), 016, (2005). [DOI], [astro-ph/0411606]. (Cited on pages 24, 25, and 102.)

[163] Dudas, E., "Theory and phenomenology of type I strings and M theory", Class. Quantum Grav., 17, R41, (2000). [DOI]. (Cited on page 91.)

[164] Duff, M.J., "Comment on time-variation of fundamental constants", arxiv, e-print, (2002). [arxiv:hep-th/0208093]. (Cited on page 17.)

[165] Duff, M.J., Okun, L.B. and Veneziano, G., "Trialogue on the number of fundamental constants", J. High Energy Phys., 2002(03), 023, (2002). [DOI], [physics/0110060]. (Cited on pages 9 and 16.)

[166] Dvali, G. and Zaldarriaga, M., "Changing $\alpha$ with Time: Implications For Fifth-Force-Type Experiments and Quintessence", Phys. Rev. Lett., 88, 091303, (2002). [DOI], [hep-ph/0108217]. (Cited on pages 22, 24, and 108.)

[167] Dyson, F.J., "Time variation of the charge of the proton", Phys. Rev. Lett., 19, 1291, (1967). [DOI]. (Cited on page 43.) 
[168] Dyson, F.J., "The Fundamental Constants and Their Time Variation", in Salam, A. and Wigner, E.P., eds., Aspects of Quantum Theory, pp. 213-236, (Cambridge University Press, Cambridge; New York, 1972). [Google Books]. (Cited on pages 40 and 42.)

[169] Dzuba, V.A. and Flambaum, V.V., "Atomic optical clocks and search for the variation of the fine-structure constant", Phys. Rev. A, 61, 034502, (2000). [DOI]. (Cited on page 44.)

[170] Dzuba, V.A. and Flambaum, V.V., "Atomic clocks and search for variation of the fine structure constant", Phys. Rev. A, 61, 034502, (2001). [DOI]. (Cited on page 28.)

[171] Dzuba, V.A. and Flambaum, V.V., "Fine-structure and search of variation of the finestructure constant in laboratory experiments", Phys. Rev. A, 72, 052514, (2005). [DOI], [physics/0510072]. (Cited on page 33.)

[172] Dzuba, V.A. and Flambaum, V.V., "Sensitivity of the energy levels of singly ionized cobalt to the variation of the fine structure constant", Phys. Rev. A, 81, 034501, (2010). [DOI], [arXiv:1002.1750 [astro-ph.CO]]. (Cited on page 60.)

[173] Dzuba, V.A. and Flambaum, V.V., "Theoretical study of the experimentally important states of dysprosium", Phys. Rev. A, 81, 052515, (2010). [DOI], [arXiv:1003.1184 [physics.atom-ph]]. (Cited on page 31.)

[174] Dzuba, V.A., Flambaum, V.V. and Marchenko, M.V., "Relativistic effect in Sr, Dy, YbII, and YbIII and search for variation of the fine structure constant", Phys. Rev. A, 68, 022506, (2003). [DOI], [physics/0305066]. (Cited on pages 28 and 31.)

[175] Dzuba, V.A., Flambaum, V.V. and Webb, J.K., "Calculations of the relativistic effects in many electron atoms and space-time variation of fundamental constants", Phys. Rev. A, 59, 230, (1999). [DOI], [physics/9808021]. (Cited on pages 28, 31, 44, and 45.)

[176] Dzuba, V.A., Flambaum, V.V. and Webb, J.K., "Space-time variation of physical constants and relativistic corrections in atoms", Phys. Rev. Lett., 82, 888, (1999). [DOI]. (Cited on page 47.)

[177] Eardley, D.M., "Observable effects of a scalar gravitational field in a binary pulsar", Astrophys. J. Lett., 196, L59-L62, (1975). [DOI], [ADS]. (Cited on pages 77 and 78.)

[178] Eddington, A., Relativity Theory of Protons and Electrons, (Cambridge University Press, Cambridge, 1936). (Cited on page 110.)

[179] Eddington, A., Fundamental Theory, (Cambridge University Press, Cambridge, 1948). (Cited on page 110.)

[180] Ekström, S., Coc, A., Descouvemont, P., Meynet, G., Olive, K.A., Uzan, J.-P. and Vangioni, E., "Effects of the variation of fundamental constants on Population III stellar evolution", Astron. Astrophys., 514, A62, (2010). [DOI], [arXiv:0911.2420 [astro-ph.SR]]. (Cited on page 63.)

[181] Ekström, S., Meynet, G., Chiappini, C., Hirschi, R. and Maeder, A., "Effects of rotation on the evolution of primordial stars", Astron. Astrophys., 489, 685, (2008). [DOI], [arXiv:0807.0573 [astro-ph]]. (Cited on page 63.)

[182] Ellis, G.F.R., Kirchner, U. and Stoeger, W.R., "Multiverses and physical cosmology", Mon. Not. R. Astron. Soc., 34, 921, (2004). [DOI], [astro-ph/0305292]. (Cited on page 113.)

[183] Ellis, G.F.R. and Uzan, J.-P., "c' is the speed of light, isn't it?", Am. J. Phys., 73, 240-247, (2005). [DOI], [gr-qc/0305099]. (Cited on pages 14, 86, and 101.) 
[184] Ellis, J., Ibáñez, L. and Ross, G.G., "Grand Unification with Large Supersymmetry Breaking", Phys. Lett. B, 113, 283-287, (1982). [DOI]. (Cited on page 93.)

[185] Ellis, J., Ibáñez, L. and Ross, G.G., "SU(2) $\mathrm{L} \times \mathrm{U}(1)$ Symmetry Breaking as a Radiative Effect of Supersymmetry Breaking in Guts", Phys. Lett. B, 110, 215-220, (1982). [DOI]. (Cited on page 93.)

[186] Ellis, J., Kalara, S., Olive, K.A. and Wetterich, C., "Density-dependent couplings and astrophysical bounds on light scalar particles", Phys. Lett. B, 228, 264, (1989). (Cited on page 99.)

[187] Ellison, S.L., Ryan, S.G. and Prochaska, J.X., "The first detection of cobalt in a damped Lyman alpha system", Mon. Not. R. Astron. Soc., 326, 628, (2001). [DOI], [astro-ph/0104301]. (Cited on page 60.)

[188] Epelbaum, E., Meissner, U.G. and Glöckle, W., "Nuclear forces in the chiral limit", Nucl. Phys. A, 714, 535-574, (2003). [DOI], [nucl-th/0207089]. (Cited on pages 74 and 95.)

[189] Esposito-Farèse, G., "Tests of Alternative Theories of Gravity", in Hewett, J., Jaros, J., Kamae, T. and Prescott, C., eds., Gravity in the Quantum World and the Cosmos, Proceedings of the 33rd SLAC Summer Institute on Particle Physics (SSI 2005), Menlo Park, USA, 25 July -5 August 2005, 819, (SLAC, Stanford, 2005). URL (accessed 27 September 2010): http://www.slac.stanford.edu/econf/C0507252/papers/T025.PDF. (Cited on pages 20 and 87.)

[190] Esposito-Farèse, G., "Motion in alternative theories of gravity", in Blanchet, L., Spallicci, A. and Whiting, B., eds., Mass and Motion in General Relativity, Lectures from the CNRS School on Mass held in Orléans, France, 23-25 June 2008, Fundamental Theories of Physics, 162, pp. 461-489, (Springer, Berlin; New York, 2011). [DOI], [arXiv:0905.2575 [gr-qc]]. (Cited on page 18.)

[191] Esposito-Farèse, G. and Polarski, D., "Scalar-tensor gravity in an accelerating universe", Phys. Rev. D, 63, 063504, (2001). [DOI], [gr-qc/0009034]. (Cited on page 86.)

[192] Fenner, Y., Murphy, M.T. and Gibson, B.K., "On variations in the fine-structure constant and stellar pollution of quasar absorption systems", Mon. Not. R. Astron. Soc., 358, 468, (2005). [DOI], [astro-ph/0501168]. (Cited on page 49.)

[193] Ferrel, S.J. et al., "Investigation of the gravitational potential dependence of the finestructure constant using atomic dyprosium", Phys. Rev. A, 76, 062104, (2007). [DOI], [arXiv:0708.0569 [physics.atom-ph]]. (Cited on page 104.)

[194] Ferrero, A. and Altschul, B., "Limits on the Time Variation of the Fermi Constant $G_{\mathrm{F}}$ Based on Type Ia Supernova Observations", Phys. Rev. D, 82, 123002, 1-8, (2010). [DOI], [arXiv:1008.4769 [hep-ph]]. (Cited on page 81.)

[195] Fierz, M., "On the physical interpretation of P. Jordan's extended theory of gravitation", Helv. Phys. Acta, 29, 128, (1956). (Cited on pages 7, 85, and 100.)

[196] Fischer, M. et al., "New limits on the drift of fundamental constants from laboratory measurements", Phys. Rev. Lett., 92, 230802, (2004). [DOI], [physics/0312086]. (Cited on pages 29 and 30.)

[197] Flambaum, V.V., "Limits on temporal variation of quark masses and strong interaction from atomic clock experiments", arxiv, e-print, (2003). [arxiv:physics/0302015]. (Cited on page 96.) 
[198] Flambaum, V.V., "Limits on temporal variation of fine structure constant, quark masses and strong interaction from atomic clock experiments", in Hannaford, P., Sidorov, A., Bachor, H. and Baldwin, K., eds., Laser Spectroscopy, Proceedings of the XVI International Conference, Palm Cove, Australia, 13-18 July 2003, pp. 49-57, (World Scientific, Singapore, 2004). [physics/0309107]. (Cited on pages 28, 32, and 33.)

[199] Flambaum, V.V., "Enhanced effect of temporal variation of the fine-structure constant and the strong interaction in ${ }^{229} \mathrm{Th} "$, Phys. Rev. Lett., 97, 092502, (2006). [DOI], [physics/0604188]. (Cited on pages 33 and 34.)

[200] Flambaum, V.V. and Dzuba, V.A., "Search for variation of the fundamental constants in atomic, molecular and nuclear spectra", Can. J. Phys., 87, 25, (2009). [DOI], [arXiv:0805.0462 [physics.atom-ph]]. (Cited on page 33.)

[201] Flambaum, V.V. and Kozlov, M.G., "Enhanced sensitivity to time-variation of $m_{p} / m_{e}$ in the inversion spectrum of ammonia", Phys. Rev. Lett., 98, 240801, (2007). [DOI], [arXiv:0704.2301 [astro-ph]]. (Cited on page 57.)

[202] Flambaum, V.V. and Kozlov, M.G., "Enhanced sensitivity to variation of the fine structure constant and $m_{p} / m_{e}$ in diatomic molecules", Phys. Rev. Lett., 99, 150801, (2007). [DOI], [arXiv:0705.0849 [physics.atom-ph]]. (Cited on pages 33 and 57.)

[203] Flambaum, V.V., Lambert, S. and Pospelov, M., "Scalar-tensor theories with pseudo-scalar couplings", Phys. Rev. D, 80, 105021, (2009). [DOI], [arXiv:0902.3217 [hep-ph]]. (Cited on page 101.)

[204] Flambaum, V.V., Leinweber, D.B., Thomas, A.W. and Young, R.D., "Limits on the temporal variation of the fine structure constant, quark masses and strong interaction from quasar absorption spectra and atomic clock experiments", Phys. Rev. D, 69, 115006, (2004). [hepph/0402098]. (Cited on pages 33, 95, and 96.)

[205] Flambaum, V.V. and Porsev, S.G., "Enhanced sensitivity to the fine-structure constant variation in Th IV atomic clock transition", Phys. Rev. A, 80, 064502, (2009). [DOI], [arXiv:0910.3459 [physics.atom-ph]]. (Cited on page 33.)

[206] Flambaum, V.V. and Porsev, S.G., "Comment on '21-cm Radiation: A New Probe of Variation in the Fine-Structure Constant"', Phys. Rev. Lett., 105, 039001, (2010). [DOI], [arXiv:1004.2540 [astro-ph.CO]]. (Cited on page 68.)

[207] Flambaum, V.V. and Shuryak, E.V., "Limits on cosmological variation of strong interaction and quark masses from big bang nucleosynthesis, cosmic, laboratory and Oklo data", Phys. Rev. D, 65, 103503, (2002). [DOI], [hep-ph/0201303]. (Cited on pages 39, 71, 73, and 74.)

[208] Flambaum, V.V. and Shuryak, E.V., "Dependence of hadronic properties on quark and constraints on their cosmological variation", Phys. Rev. D, 67, 083507, (2003). [DOI], [hepph/0212403]. (Cited on pages 22, 40, 71, 74, and 95.)

[209] Flambaum, V.V. and Shuryak, E.V., "How changing physical constants and violation of local position invariance may occur?", in Danielewicz, P., Piecuch, P. and Zelevinsky, V., eds., Nuclei and Mesoscopic Physics, Workshop in East Lansing (Michigan), 20-22 October 2007, AIP Conference Proceedings, 995, pp. 1-11, (American Institute of Physics, Melville, NY, 2008). [DOI], [physics/0701220]. (Cited on pages 104 and 105.) 
[210] Flambaum, V.V. and Tedesco, A.F., "Dependence of nuclear magnetic moments on quark masses and limits on temporal variation of fundamental constants from atomic clock experiments", Phys. Rev. C, 73, 055501, (2006). [DOI], [nucl-th/060150]. (Cited on pages 28, 32, 33 , and 105.)

[211] Flambaum, V.V. and Wiringa, R.B., "Dependence of nuclear binding on hadronic mass variation", Phys. Rev. C, 76, 054002, (2007). [DOI], [arXiv:0709.0077 [nucl-th]]. (Cited on page 96.$)$

[212] Flambaum, V.V. and Wiringa, R.B., "Enhanced effect of quark mass variation in ${ }^{229} \mathrm{Th}$ and limits from Oklo data", Phys. Rev. C, 79, 034302, (2009). [DOI], [arXiv:0807.4943 [nucl-th]]. (Cited on page 39.)

[213] Flowers, J.L. and Petley, B.W., "Progress in our knowledge of the fundamental constants of physics", Rep. Prog. Phys., 64, 1191, (2001). [DOI]. (Cited on pages 10 and 13.)

[214] Fortier, T.M. et al., "Precision atomic spectroscopy for improved limits on variation of the fine structure constant and local position invariance", Phys. Rev. Lett., 98, 070801, (2007). [DOI]. (Cited on pages 29, 30, and 104.)

[215] Fritzsch, H., The fundamental constants, a mistery of physics, (World Scientific, Singapore, 2009). (Cited on page 9.)

[216] Fritzsch, H., "The Fundamental Constants in Physics", Phys. Usp., 52, 359, (2009). [DOI], [arXiv:0902.2989 [hep-ph]]. (Cited on page 9.)

[217] Fujii, Y., "Accelerating universe and the time-dependent fine-structure constant", Mem. Soc. Astron. Ital., 80, 780, (2009). (Cited on page 102.)

[218] Fujii, Y. and Iwamoto, A., "Re/OS constraint on the time variability of the fine structure constant", Phys. Rev. Lett., 91, 261101, (2003). [DOI], [hep-ph/0309087]. (Cited on pages 41 and 44.)

[219] Fujii, Y. and Iwamoto, A., "How strongly does dating meteorites constrain the timedependence of the fine-structure constant?", Mod. Phys. Lett. A, 20, 2417-2434, (2005). [DOI], [hep-ph/0508072]. (Cited on pages 41 and 44.)

[220] Fujii, Y., Iwamoto, A., Fukahori, T., Ohnuki, T., Nakagawa, M., Hidaka, H., Oura, Y. and Möller, P., "The nuclear interaction at Oklo 2 billion years ago", Nucl. Phys. B, 573, 377, (2000). [DOI], [hep-ph/9809549]. (Cited on pages 37, 38, and 39.)

[221] Furlanetto, S.R., Oh, S.P. and Briggs, F.H., "Cosmology at low frequencies: The $21 \mathrm{~cm}$ transition and the high-redshift universe", Phys. Rep., 433, 181, (2006). [DOI], [astro-ph/0608032]. (Cited on pages 66, 67, and 68.)

[222] Furnstahl, R.J. and Serot, B.D., "Parameter counting in relativistic mean-field models", Nucl. Phys. A, 671, 447, (2000). [DOI], [nucl-th/9911019]. (Cited on page 95.)

[223] Gambini, R. and Pullin, J., "Discrete Quantum Gravity: A Mechanism for Selecting the Value of Fundamental Constants", Int. J. Mod. Phys. D, 12, 1775-1781, (2003). [DOI], [grqc/0306095]. (Cited on page 101.)

[224] Gamow, G., "Electricity, gravity and cosmology", Phys. Rev. Lett., 19, 759, (1967). (Cited on pages 63,76 , and 79.) 
[225] García-Berro, E., Hernanz, M., Isern, J. and Mochkovitch, R., "The rate of change of the gravitational constant and the cooling of white dwarfs", Mon. Not. R. Astron. Soc., 277, 801-810, (1995). [ADS]. (Cited on page 81.)

[226] García-Berro, E., Isern, J. and Kubyshin, Y.A., "Astronomical measurements and constraints on the variability of fundamental constants", Astron. Astrophys. Rev., 14, 113-170, (2007). [DOI], [astro-ph/0409424]. (Cited on page 8.)

[227] García-Berro, E., Kubyshin, Y., Loren-Aguilar, P. and Isern, J., "The variation of the gravitational constant inferred from the Hubble diagram of Type Ia supernovae", Int. J. Mod. Phys. D, 15, 1163-1174, (2006). [DOI], [gr-qc/0512164]. (Cited on page 81.)

[228] Garriga, J. and Vilenkin, A., "On likely values of the cosmological constant", Phys. Rev. D, 61, 083502, (2000). [DOI], [astro-ph/9908115]. (Cited on page 113.)

[229] Gasperini, M., Piazza, F. and Veneziano, G., "Quintessence as a runaway dilaton", Phys. Rev. D, 65, 023508, (2002). [DOI]. (Cited on pages 23 and 99.)

[230] Gasser, J. and Leutwyler, H., "Quark Masses", Phys. Rep., 87, 77, (1982). [DOI]. (Cited on pages 22 and 73.)

[231] Gay, P.L. and Lambert, D.L., "The Isotopic Abundances of Magnesium in Stars", Astrophys. J., 533, 260, (2000). [DOI], [astro-ph/9911217]. (Cited on page 49.)

[232] Gaztañaga, E., García-Berro, E., Isern, J., Bravo, E. and Dominguez, I., "Bounds On The Possible Evolution Of The Gravitational Constant From Cosmological Type Ia Supernovae", Phys. Rev. D, 65, 023506, (2002). (Cited on page 81.)

[233] Goldman, I., "Upper limit on $G$ variability derived from the spin-down of PSR 0655+64", Mon. Not. R. Astron. Soc., 244, 184-187, (1990). [ADS]. (Cited on page 78.)

[234] Gould, C.R., Sharapov, E.I. and Lamoreaux, S.K., "Time-variability of $\alpha$ from realistic models of Oklo reactors", Phys. Rev. C, 74, 024607, (2006). [DOI], [nucl-ex/0701019]. (Cited on pages 37,38 , and 39.)

[235] Granda, L.N. and Escobar, L.D., "Holographic dark energy with non-minimal coupling", arXiv, e-print, (2009). [arXiv:0910.0515 [hep-th]]. (Cited on page 102.)

[236] Griest, K., Whitmore, J.B., Wolfe, A.M., Prochaska, J.X., Howk, J.C. and Marcy, G.W., "Wavelengths accuracy of the Keck HIRES spectrograph and measuring changes in the fine structure constant", Astrophys. J., 708, 158, (2010). [DOI], [arXiv:0904.4725 [astro-ph.CO]]. (Cited on pages 48 and 50.)

[237] Gross, D.J. and Sloan, J.H., "The Quartic Effective Action for the Heterotic String", Nucl. Phys. B, 291, 41-89, (1987). [DOI]. (Cited on page 90.)

[238] Grupe, D., Pradhan, A.K. and Frank, S., "Studying the variation of the fine structure constant using emission-line multiplets", Astron. J., 130, 355, (2005). [DOI], [astro-ph/0504027]. (Cited on page 58.)

[239] Guenther, D.B., Krauss, L.M. and Demarque, P., "Testing the Constancy of the Gravitational Constant Using Helioseismology", Astrophys. J., 498, 871-876, (1998). [DOI]. (Cited on page 80.) 
[240] Guenther, D.B., Sills, K., Demarque, P. and Krauss, L.M., "Sensitivity of solar $g$-modes to varying $G$ cosmologies", Astrophys. J., 445, 148-151, (1995). [DOI], [ADS]. (Cited on page 80 .)

[241] Gundlach, J.H. and Merkowitz, S.M., "Measurement of Newton's Constant Using a Torsion Balance with Angular Acceleration Feedback", Phys. Rev. Lett., 85, 2869, (2000). [DOI]. (Cited on page 76.)

[242] Gurzadyan, V.G. et al., "A new limit on the light speed isotropy from the GRAAL experiment at the ESRF", arXiv, e-print, (2010). [arXiv:1004.2867 [physics.acc-ph]]. (Cited on page 22.)

[243] Hall, L.J. and Nomura, Y., "Evidence for the Multiverse in the Standard Model and Beyond", Phys. Rev. D, 78, 035001, (2008). [DOI], [arXiv:0712.2454 [hep-ph]]. (Cited on page 112.)

[244] Hannestad, S., "Possible constraints on the time variation of the fine structure constant from cosmic microwave background data", Phys. Rev. D, 60, 023515, (1999). [DOI], [astroph/9810102]. (Cited on page 65.)

[245] Harnik, R., Kribs, G.D. and Perez, G., "A Universe Without Weak Interactions", Phys. Rev. $D, \mathbf{7 4}, 035006,(2006)$. [DOI], [hep-ph/0604027]. (Cited on page 111.)

[246] Haugan, M.P. and Will, C.M., "Weak Interactions and Eötvös Experiments", Phys. Rev. Lett., 37, 1, (1976). [DOI]. (Cited on page 21.)

[247] Hayes, A.C. and Friar, J.L., "Sensitivity of nuclear transition frequencies to temporal variation of the fine structure constant or the strong interaction", Phys. Lett. B, 650, 229, (2007). [nucl-th/0702048]. (Cited on page 34.)

[248] Heintzmann, H. and Hillebrandt, H., "Pulsar slow-down and the temporal change of $G$ ", Phys. Lett. A, 54, 349, (1975). [DOI]. (Cited on page 78.)

[249] Hellings, R.W., Adams, P.J., Anderson, J.D., Keesey, M.S., Lau, E.L., Standish, E.M., Canuto, V.M. and Goldman, I., "Experimental Test of the Variability of $G$ Using Viking Lander Ranging Data", Phys. Rev. Lett., 51, 1609-1612, (1983). [DOI]. (Cited on page 77.)

[250] Henkel, C. et al., "The density, the cosmic microwave background, and the proton-toelectron mass ratio in a cloud at redshift 0.9", Astron. Astrophys., 500, 745, (2009). [DOI], [arXiv:0904.3081 [astro-ph.CO]]. (Cited on pages 57 and 59.)

[251] Hill, H.A. and Gu, Y.-M., "Extension of range in radial order in detection and mode classification of solar low-degree gravity modes", Sci. China Ser. A, 33, 854-866, (1990). (Cited on page 80.)

[252] Hogan, C.J., "Why the universe is just so", Rev. Mod. Phys., 72, 1149-1161, (2000). [DOI], [astro-ph/9909295]. (Cited on pages 7 and 111.)

[253] Hogan, C.J., "Nuclear astrophysics of worlds in the string landscape", Phys. Rev. D, 74, 123514, (2006). [DOI], [astro-ph/0602104]. (Cited on page 111.)

[254] Hogan, C.J., "Quarks, electrons and atoms in closely related universes", in Carr, B.J., ed., Universe or Multiverse?, pp. 221-230, (Cambridge University Press, Cambridge; New York, 2007). [astro-ph/0407086], [Google Books]. (Cited on page 111.)

[255] Hořava, P. and Witten, E., "Heterotic and type I string dynamics from eleven-dimension", Nucl. Phys. B, 460, 506-524, (1996). [DOI], [hep-th/9510209]. (Cited on page 91.) 
[256] Hoyle, C.D., Kapner, D.J., Heckel, B.R., Adelberger, E.G., Gundlach, J.H., Schmidt, U. and Swanson, H.E., "Submillimeter tests of the gravitational inverse-square law", Phys. Rev. D, 70, 042004, (2004). [DOI], [hep-ph/0405262]. (Cited on page 20.)

[257] Hoyle, F., "On nuclear reactions occuring in very hot stars. I. The synthesis of elements from carbon to nickel", Astrophys. J. Suppl. Ser., 1, 121, (1954). [DOI]. (Cited on page 61.)

[258] Hoyle, F., Galaxies, Nuclei and Quasars, (Harper \& Row, New York, 1965). (Cited on page 110.)

[259] Ichikawa, K., Kanzaki, T. and Kawasaki, M., "CMB constraints on the simultaneous variation of the fine structure constant and electron mass", Phys. Rev. D, 74, 023515, (2006). [DOI], [astro-ph/0602577]. (Cited on pages 66 and 67.)

[260] Ichikawa, K. and Kawasaki, M., "Big bang nucleosynthesis with a varying fine structure constant and non standard expansion rate", Phys. Rev. D, 69, 123506, (2005). [hep-ph/0401231]. (Cited on page 70.)

[261] Ivanchik, A., Petitjean, P., Varshalovich, D., Aracil, B., Srianand, R., Chand, H., Ledoux, C. and Boissé, P., "A new constraint on the time dependence of the proton-to-electron mass ratio: Analysis of the Q 0347-383 and Q 0405-443 spectra", Astron. Astrophys., 440, 45-52, (2005). [DOI], [astro-ph/0507174]. (Cited on page 56.)

[262] Ivanchik, A., Rodriguez, E., Petitjean, P. and Varshalovich, D., "Do the fundamental constants vary in the course of the cosmological evolution?", Astron. Lett., 28, 423, (2002). [DOI], [astro-ph/0112323]. (Cited on page 55.)

[263] Ivanov, T.L., Roudjane, M., Vieitez, M.O., de Lange, C.A., Tchang-Brillet, W.-Ü.L. and Ubachs, W., "HD as a Probe for Detecting Mass Variation on a Cosmological Time Scale", Phys. Rev. Lett., 100, 093007, (2009). [DOI]. (Cited on page 57.)

[264] Jaffe, R.L., Jenkins, A. and Kimchi, I., "Quark Masses: An Environmental Impact Statement", Phys. Rev. D, 79, 065014, (2009). [DOI], [arXiv:0809.1647 [hep-ph]]. (Cited on page 111.)

[265] Jenkins, J.H., Fischbach, E., Buncher, J.B., Gruenwald, J.T., Krause, D.E. and Mattes, J.J., "Evidence for correlations between nuclear decay rates and Earth-Sun distance", Astropart. Phys., 32, 42, (2009). [DOI], [arXiv:0808.3283 [astro-ph]]. (Cited on page 105.)

[266] Jofré, P., Reisenegger, A. and Fernández, R., "Constraining a possible time-variation of the gravitational constant through 'gravitochemical heating' of neutron stars", Phys. Rev. Lett., 97, 131102, (2006). [DOI], [astro-ph/0606708]. (Cited on page 79.)

[267] Johnstone-Stoney, G., "On the physical units of nature", Philos. Mag., 5, 381, (1881). (Cited on page 15.)

[268] Jordan, P., "Die physikalischen Weltkonstanten", Die Naturwissenschaften, 25, 513-517, (1937). [DOI]. (Cited on pages 7 and 85.)

[269] Kaluza, T., "Zum Unitätsproblem in der Physik", Sitzungsber. Preuss. Akad. Wiss., 1921, 966-972, (1921). (Cited on page 89.)

[270] Kane, G.L., Perry, M.J. and Zytkow, A.N., "The beginning of the end of the anthropic principle", New Astronomy, 7, 45-53, (2002). [DOI]. (Cited on page 110.) 
[271] Kanekar, N., "Probing fundamental constant evolution with radio spectroscopy", Mem. Soc. Astron. Ital., 80, 895, (2009). [ADS]. (Cited on page 44.)

[272] Kanekar, N. and Chengalur, J.N., "The use of OH 'main' lines to constrain the variation of fundamental constants", Mon. Not. R. Astron. Soc., 350, L17, (2004). [DOI], [astroph/0310765]. (Cited on page 53.)

[273] Kanekar, N., Chengalur, J.N. and Ghosh, T., "Probing fundamental constant evolution with redshifted conjugate-satellite OH lines", Astrophys. J., 716, L23, (2010). [DOI], [arXiv:1004.5383 [astro-ph.CO]]. (Cited on pages 54 and 59.)

[274] Kanekar, N., Prochaska, J.X., Ellison, S.L. and Chengalur, J.N., "Probing fundamental constant evolution with neutral atomic gas lines", Astrophys. J., 712, 148, (2010). [arXiv:1003.0444 [astro-ph.CO]]. (Cited on page 52.)

[275] Kanekar, N., Subrahmanyan, R., Ellison, S.L., Lane, W.M. and Chengalur, J.N., "H i 21 cm absorption at $z \sim 2.347$ towards PKS B0438-436", Mon. Not. R. Astron. Soc., 370, L46-L50, (2006). [DOI], [astro-ph/0605346]. (Cited on page 52.)

[276] Kanekar, N. et al., "Constraints on changes in fundamental constants from a cosmologically distant OH absorber/emitter", Phys. Rev. Lett., 95, 261301, (2005). [DOI], [astro-ph/0510760]. (Cited on pages 54 and 59.)

[277] Kaplinghat, M., Scherrer, R.J. and Turner, M.S., "Constraining variations in the finestructure constant with the cosmic microwave background", Phys. Rev. D, 60, 023516, (1999). [DOI], [astro-ph/9810133]. (Cited on page 65.)

[278] Karshenboim, S.G., "Fundamental physical constants: looking from different angles", Can. J. Phys., 83, 767, (2005). [DOI], [physics/0506173]. (Cited on pages 8, 13, and 14.)

[279] Karshenboim, S.G., "On a natural definition of the kilogram and the ampere: the objectives and consequences", e-print, (2005). [physics/0507200]. (Cited on page 15.)

[280] Karshenboim, S.G., "Precision physics of simple atoms: QED tests, nuclear structure and fundamental constants", Phys. Rep., 422, 1, (2005). [DOI], [hep-ph/0509010]. (Cited on pages 13 and 28.)

[281] Karshenboim, S.G., "The search for possible variation of the fine structure constant", Gen. Relativ. Gravit., 38, 159, (2006). [DOI], [physics/0311080]. (Cited on page 8.)

[282] Kaspi, V.M., Taylor, J.H. and Riba, M.F., "High-precision timing of millisecond pulsars. III. Long-term monitoring of PSRs B1855+09 and B1937+21", Astrophys. J., 428, 713-728, (1994). [DOI], [ADS]. (Cited on page 78.)

[283] Ketchum, J.A. and Adams, F.C., "The future evolution of white dwarf stars through baryon decay and time varying gravitational constant", Astrophys. Space Sci., 317(3-4), 221-230, (2008). [DOI], [arXiv:0808.1301 [astro-ph]]. (Cited on page 80.)

[284] Khatri, R. and Wandelt, B., "21-cm Radiation: A New Probe of Variation in the FineStructure Constant", Phys. Rev. Lett., 98, 111201, (2007). [DOI], [astro-ph/0701752]. (Cited on pages 66,67 , and 68.)

[285] Khatri, R. and Wandelt, B.D., "21cm radiation: a new probe of fundamental physics", Mem. Soc. Astron. Ital., 80, 824, (2009). [arXiv:0910.2710 [astro-ph.CO]]. (Cited on page 67.) 
[286] Khatri, R. and Wandelt, B.D., "Reply", Phys. Rev. Lett., 105, 039002, (2010). [DOI], [arXiv:1007.1963 [astro-ph.CO]]. (Cited on page 68.)

[287] Khoury, J. and Weltman, A., "Chameleon cosmology", Phys. Rev. D, 69, 044026, (2004). [DOI], [astro-ph/0309300]. (Cited on pages 87 and 99.)

[288] Khoury, J. and Weltman, A., "Chameleon Fields: Awaiting Surprises for Tests of Gravity in Space", Phys. Rev. Lett., 93, 171104, (2004). [DOI], [astro-ph/0309411]. (Cited on page 99.)

[289] King, J.A., Webb, J.K., Murphy, M.T. and Carswell, R.F., "Stringent null constraint on cosmological evolution of the proton-to-electron mass ratio", Phys. Rev. Lett., 101, 251304, (2008). [DOI], [arXiv:0807.4366 [astro-ph]]. (Cited on pages 56 and 59.)

[290] Kiritsis, E., "Supergravity, D-brane probes and thermal super Yang-Mills: A comparison", $J$. High Energy Phys., 1999(10), 010, (1999). [DOI], [arXiv:hep-th/9906206]. (Cited on page 91.)

[291] Klein, O., "Quantum Theory and Five-Dimensional Relativity Theory", Z. Phys., 37, 894906, (1926). [DOI]. Reprinted in Surveys High Energ. Phys., 5, 241-244, (1986). (Cited on page 89.)

[292] Kneller, J.P. and Steigman, G., "Big bang nucleosynthesis and CMB constraints on dark energy", Phys. Rev. D, 67, 063501, (2003). [DOI]. (Cited on page 83.)

[293] Kofman, L., "Probing String Theory with Modulated Cosmological Fluctuations", e-print, (2003). [astro-ph/0303614]. (Cited on page 107.)

[294] Kofman, L., Bernardeau, F. and Uzan, J.-P., "Modulated fluctuations from hybrid inflation", Phys. Rev. D, 70, 083004, (2004). [astro-ph/0403315]. (Cited on page 107.)

[295] Kolb, E.W., Perry, M.J. and Walker, T.P., "Time variation of fundamental constants, primordial nucleosynthesis and the size of extra dimensions", Phys. Rev. D, 33, 869, (1986). [DOI]. (Cited on pages 70, 84, and 89.)

[296] Komatsu, E. et al. (WMAP Collaboration), "Five-year Wilkinson Microwave Anisotropy Probe (WMAP) observations: cosmological interpretation", Astrophys. J. Suppl. Ser., 180, 330-376, (2009). [DOI], [arXiv:0803.0547 [astro-ph]]. (Cited on pages 24 and 69.)

[297] Korennov, S. and Descouvemont, P., "A microscopic three-cluster model in the hyperspherical formalism", Nucl. Phys. A, 740, 249, (2004). [DOI]. (Cited on page 62.)

[298] Kostelecký, V.A., Lehnert, R. and Perry, M.J., "Spacetime-varying couplings and Lorentz violation", Phys. Rev. D, 68, 123511, (2003). [DOI], [arXiv:astro-ph/0212003]. (Cited on page 22.)

[299] Kozlov, M.G., Lapinov, A.V. and Levshakov, S.A., "Sensitivity of microwave and FIR spectra to variation of fundamental constants", Mem. Soc. Astron. Ital., 80, 901-904, (2009). [arXiv:0910.4799]. (Cited on page 54.)

[300] Kozlov, M.G., Porsev, S.G., Levshakov, S.A., Reimers, D. and Molaro, P., "Mid- and far-infrared fine-structure line sensitivities to hypothetical variability of the fine-structure constant", Phys. Rev. A, 77, 032119, (2008). [DOI], [arXiv:0802.0269 [astro-ph]]. (Cited on page 54.)

[301] Kraiselburd, L. and Vucetich, H., "Violation of the weak equivalence principle in Bekenstein's theory", Int. J. Mod. Phys. E, 20, 101-111, (2011). [DOI], [arXiv:0902.4146 [gr-qc]]. (Cited on page 100.) 
[302] Krastev, P.G. and Li, A.-A., "Constraining a possible time variation of the gravitational constant $G$ with terrestrial nuclear laboratory data", Phys. Rev. C, 76, 055804, (2007). [DOI], [nucl-th/0702080]. (Cited on page 79.)

[303] Kuroda, P.K., "On the nuclear physical stability of uranium mineral", J. Chem. Phys., 25, 781, (1956). [DOI]. (Cited on page 34.)

[304] Lamoreaux, S.K., Jacobs, J.P., Heckel, B.R., Raab, F.J. and Fortson, E.N., "New limits on spatial anisotropy from optically-pumped ${ }^{201} \mathrm{Hg}$ and ${ }^{199} \mathrm{Hg}$ ", Phys. Rev. Lett., 57, 3125-3128, (1986). [DOI]. (Cited on page 18.)

[305] Lamoreaux, S.K. and Togerson, J.R., "Neutron moderation in the Oklo natural reactor and the time variation of $\alpha$ ", Phys. Rev. D, 69, 12170, (2004). [DOI], [nucl-th/0309048]. (Cited on pages 37 and 38.)

[306] Landau, S.J., Bersten, M., Sisterna, P. and Vucetich, H., "Testing a String Dilaton Model with Experimental and Observational Data", in Grece, S.A., ed., New Developments in String Theory Research, pp. 153-173, (Nova Science, New York, 2006). [astro-ph/0410030], [Google Books]. (Cited on page 97.)

[307] Landau, S.J., Harari, D.D. and Zaldarriaga, M., "Constraining non-standard recombination: A worked example", Phys. Rev. D, 63, 083505, (2001). [DOI], [astro-ph/0010415]. (Cited on pages 65 and 67.)

[308] Landau, S.J., Mosquera, M.E., Scoccola, C.G. and Vucetich, H., "Early Universe Constraints on Time Variation of Fundamental Constants", Phys. Rev. D, 78, 083527, (2008). [DOI], [arXiv:0809.2033 [astro-ph]]. (Cited on page 70.)

[309] Landau, S.J., Mosquera, M.E. and Vucetich, H., "Primordial nucleosynthesis with varying of fundamental constants: a semi-analytical approach", Astrophys. J., 637, 38, (2006). [DOI], [astro-ph/0411150]. (Cited on page 71.)

[310] Landau, S.J. and Scóccola, C.G., "Constraints on variation in $\alpha$ and $m_{e}$ from WMAP 7-year data", Astron. Astrophys., 517, A62, (2010). [DOI], [arXiv:1002.1603 [astro-ph.CO]]. (Cited on pages 66 and 67.$)$

[311] Landau, S.J. and Vucetich, H., "Testing theories that predict time variation of fundamental constants", Astrophys. J., 570, 463, (2002). [DOI], [astro-ph/0005316]. (Cited on page 89.)

[312] Langacker, P., "Time variation of fundamental constants as a probe of new physics", Int. J. Mod. Phys. A, 19S1, 157-166, (2004). [DOI], [hep-ph/0304093]. (Cited on page 93.)

[313] Langacker, P., Segre, G. and Strassler, M.J., "Implications of Gauge Unification for Time Variation of the Fine Structure Constant", Phys. Lett. B, 528, 121, (2002). [hep-ph/0112233]. (Cited on page 93.)

[314] Lee, D.-S., Lee, W. and Ng, K.-W., "Bound on the time variation of the fine structure constant driven by quintessence", Int. J. Mod. Phys. D, 14, 335, (2005). [astro-ph/0309316]. (Cited on pages 24 and 102.)

[315] Lee, S., "Time variation of fine structure constant and proton-electron mass ratio with quintessence", Mod. Phys. Lett. A, 22, 2003, (2007). [DOI], [astro-ph/0702063]. (Cited on pages 24 and 102.) 
[316] Leinweber, D.B., Lu, D.H. and Thomas, A.W., "Nucleon magnetic moments beyond the perturbative chiral regime", Phys. Rev. D, 60, 034014, (1999). [DOI], [hep-lat/981005]. (Cited on pages 95 and 96.$)$

[317] Leslie, J., Universes, (Routledge, London; New York, 1989). [Google Books]. (Cited on page 110.)

[318] Levshakov, S.A., "Astrophysical Constraints on Hypothetical Variability of Fundamental Constants", in Karshenboim, S.G. and Peik, E., eds., Astrophysics, Clocks and Fundamental Constants, 302nd WE-Heraeus-Seminar, Bad Honnef, Germany, June 2003, Lecture Notes in Physics, 648, pp. 151-166, (Springer, Berlin, 2004). [DOI], [astro-ph/0309817]. (Cited on page 47.)

[319] Levshakov, S.A., Agafonova, I.I., Molaro, P. and Reimers, D., "Spatial and temporal variations of fundamental constants", Mem. Soc. Astron. Ital., 80, 850-858, (2009). [ADS]. (Cited on page 105.)

[320] Levshakov, S.A., Centurión, M., Molaro, P. and D'Odorico, S. etal, "Most precise single redshift bound to $\Delta \alpha / \alpha "$, Astrophys. J., 637, 38, (2006). [DOI], [astro-ph/0511765]. (Cited on page 51.)

[321] Levshakov, S.A., Centurion, M., Molaro, P. and Kostina, M.V., "VLT/UVES constraints on the carbon isotope ratio ${ }^{12} \mathrm{C} /{ }^{13} \mathrm{C}$ at $z=1.15$ toward the quasar HE 0515-4414", Astron. Astrophys., 447, L21, (2006). [DOI], [astro-ph/0602303]. (Cited on page 49.)

[322] Levshakov, S.A., Lapinov, A.V., Henkel, C., Molaro, P., Reimers, D., Kozlov, M.G. and Agafonova, I.I., "Searching for chameleon-like scalar fields with the ammonia method II. Mapping of cold molecular cores in $\mathrm{NH}_{3}$ and $\mathrm{HC}_{3} \mathrm{~N}$ lines", Astron. Astrophys., 524, A32, (2010). [DOI], [arXiv:1008.1160 [astro-ph.CO]]. (Cited on pages 99 and 105.)

[323] Levshakov, S.A., Molaro, P. and Kozlov, M.G., "On spatial variations of the electron-toproton mass ratio in the Milky Way", arXiv, e-print, (2008). [arXiv:0808.0583 [astro-ph]]. (Cited on pages 57, 99, and 105.)

[324] Levshakov, S.A., Molaro, P., Lapinov, A.V., Reimers, D., Henkel, C. and Sakai, T., "Searching for chameleon-like scalar fields with the ammonia method", Astron. Astrophys., 512, A44, (2010). [DOI], [arXiv:0911.3732 [astro-ph.CO]]. (Cited on pages 99 and 105.)

[325] Levshakov, S.A., Molaro, P., Lopez, S., D’Odorico, S., Centurión, M., Bonifacio, P., Agafonova, I.I. and Reimers, D., "A new measure of $\Delta \alpha / \alpha$ at redshift $z=1.84$ from very high resolution spectra of Q 1101-264", Astron. Astrophys., 466, 1077-1082, (2007). [DOI], [astro-ph/0703042]. (Cited on page 51.)

[326] Levshakov, S.A., Molaro, P. and Reimers, D., "Searching for spatial variations of $\alpha^{2} / \mu$ in the Milky Way", Astron. Astrophys., 516, A113, (2010). [DOI], [arXiv:1004.0783 [astro-ph.CO]]. (Cited on page 105.)

[327] Levshakov, S.A., Reimers, D., Kozlov, M.G., Porsev, S.G. and Molaro, P., "A new approach for testing variations of fundamental constants over cosmic epochs using FIR fine-structure lines", Astron. Astrophys., 479, 719, (2008). [DOI], [arXiv:0712.2890 [astro-ph]]. (Cited on page 59.)

[328] Lévy-Leblond, J.-M., "The importance of being (a) Constant", in Toraldo di Francia, G., ed., Problems in the Foundations of Physics, Proceedings of the International School of Physics 'Enrico Fermi' Course LXXII, Varenna, Italy, July 25-August 6, 1977, pp. 237-263, (NorthHolland, Amsterdam; New York, 1979). (Cited on page 11.) 
[329] Lewis, A. and Challinor, A., "The $21 \mathrm{~cm}$ angular-power spectrum from dark ages", Phys. Rev. D, 76, 083005, (2007). [DOI], [astro-ph/0702600]. (Cited on pages 67 and 68.)

[330] Li, B. and Chu, M.C., "Big bang nucleosynthesis constraints on universal extra dimensions and varying fundamental constants", Phys. Rev. D, 73, 025004, (2006). [astro-ph/0511013]. (Cited on page 89.)

[331] Li, B. and Chu, M.C., "Big bang nucleosynthesis with an evolving radion in the brane world scenario", Phys. Rev. D, 73, 023509, (2006). [DOI], [astro-ph/0511642]. (Cited on page 101.)

[332] Lichnerowicz, A., Théories Relativistes de la Gravitation et de l'Électromagnétisme: Relativité Générale et Théories Unitaires, (Masson, Paris, 1955). (Cited on page 100.)

[333] Lindner, M., Leich, D.A., Borg, R.J., Russ, G.P., Bazan, J.M., Simons, D.S. and Date, A.R., "Direct laboratory determination of the ${ }^{187}$ Re half-life", Nature, 320, 246-248, (1986). [DOI]. (Cited on page 43.)

[334] Livio, M., Hollowell, D., Weiss, A. and Truran, J.W., "On the anthropic significance of the existence of an excited state of ${ }^{12}$ C", Nature, 340, 281-284, (1989). [DOI]. (Cited on page 62.)

[335] Lopes, I. and Silk, J., "The implications for helioseismology of experimental uncertainties in Newton's constant", e-print, (2001). [astro-ph/0112310]. (Cited on page 80.)

[336] Lorén-Aguilar, P., García-Berro, E., Isern, J. and Kubyshin, Y.A., "Time variation of $G$ and $\alpha$ within models with extra dimensions", Class. Quantum Grav., 20, 3885-3896, (2003). [DOI], [astro-ph/0309722]. (Cited on pages 89 and 101.)

[337] Lugmair, G.W. and Galer, S.J., "Age and isotopic relationships among the angrite Lewis Cliff 86010 and Angra dos Reis", Geochim. Cosmochim. Acta, 56, 1673, (1992). [DOI]. (Cited on page 43.)

[338] Ma, C.-P. and Bertschinger, E., "Cosmological perturbation theory in the synchronous and conformal Newtonian gauge", Astrophys. J., 455, 7-25, (1995). [DOI], [astro-ph/9506072]. (Cited on page 64.)

[339] MacGibbon, J.H., "Black Hole Constraints on Varying Fundamental Constants", Phys. Rev. Lett., 99, 061301, (2007). [DOI]. (Cited on page 101.)

[340] Maeda, K.-I., "On time variation of fundamental constants in superstring theories", Mod. Phys. Lett. A, 3, 243-249, (1988). [DOI]. (Cited on pages 90 and 91.)

[341] Magueijo, J., "New varying speed of light theories", Rep. Prog. Phys., 66, 2025-2068, (2003). [DOI], [astro-ph/0305457]. (Cited on page 101.)

[342] Malec, A.L. et al., "New limit on a varying proton-to-electron mass ratio from high-resolution optical quasar spectra", Mem. Soc. Astron. Ital., 80, 882-887, (2009). [ADS]. (Cited on pages 56 and 59.)

[343] Maleki, L. and Prestage, J., "Search for New Physics with Atomic Clocks", in Karshenboim, S.G. and Peik, E., eds., Astrophysics, Clocks and Fundamental Constants, 302nd WE-Heraeus-Seminar, Bad Honnef, Germany, June 2003, Lecture Notes in Physics, 648, pp. 331-341, (Springer, Berlin, 2004). [DOI]. (Cited on page 105.)

[344] Mansfield, V.N., "Pulsar spin down and cosmologies with varying gravity", Nature, 261, 560, (1976). [DOI]. (Cited on page 78.) 
[345] Marciano, W.J., "Time Variation of the Fundamental 'Constants' and Kaluza-Klein Theories", Phys. Rev. Lett., 52, 489-491, (1984). [DOI]. (Cited on page 89.)

[346] Marion, H. et al., "A search for variations of fundamental constants using atomic fountain clock", Phys. Rev. Lett., 90, 150801, (2003). [DOI], [physics/0212112]. (Cited on page 29.)

[347] Marra, V. and Rosati, F., "Cosmological evolution of alpha driven by a general coupling with quintessence", J. Cosmol. Astropart. Phys., 2005(05), 011, (2005). [DOI], [astro-ph/0501515]. (Cited on pages 24, 101, and 102.)

[348] Martin, J., Schimd, C. and Uzan, J.-P., "Testing for $w<-1$ in the Solar System", Phys. Rev. Lett., 96, 061303, (2006). [DOI], [astro-ph/0510208]. (Cited on pages 24, 87, and 109.)

[349] Martínez Fiorenzano, A.F., Vladilo, G. and Bonifacio, P., "Search for $\alpha$ variation in UVES spectra: Analysis of C IV and Si IV doublets towards QSO 1101-264", Mem. Soc. Astron. Ital., 3, 252-255, (2003). [ADS], [astro-ph/0312270]. (Cited on pages 47 and 59.)

[350] Martins, C.J.A.P., Melchiorri, A., Rocha, G., Trotta, R., Avelino, P.P. and Viana, P.T.P., "WMAP constraints on varying $\alpha$ and the promise of reionization", Phys. Lett. B, 585, 29-34, (2004). [DOI], [astro-ph/0302295]. (Cited on page 65.)

[351] Martins, C.J.A.P., Menegoni, E., Galli, S., Mangano, G. and Melchiorri, A., "Varying couplings in the early universe: correlated variations of $\alpha$ and $G$ ", Phys. Rev. D, 82, 023532, (2010). [DOI], [arXiv:1001.3418 [astro-ph.CO]]. (Cited on page 66.)

[352] Menegoni, E., Galli, S., Bartlett, J., Martins, C.J.A.P. and Melchiorri, A., "New constraints on variations of the fine structure constant from CMB anisotropies", Phys. Rev. D, 80, 087302, (2009). [DOI], [arXiv:0909.3584 [astro-ph.CO]]. (Cited on pages 66 and 67.)

[353] Menten, K.M., Güsten, R., Leurini, S., Thorwirth, S., Henkel, C., Klein, B., Carilli, C.L. and Reid, M.J., "Submillimeter water and ammonia absorption by the peculiar $z \sim 0.89$ interstellar medium in the gravitational lens of the PKS 1830-211 system", Astron. Astrophys., 492, 725-730, (2008). [DOI], [arXiv:0810.2782 [astro-ph]]. (Cited on pages 57 and 59.)

[354] Mercuri, S. and Taveras, V., "Interaction of the Barbero-Immirzi Field with Matter and Pseudo-Scalar Perturbations", Phys. Rev. D, 80, 104007, (2009). [DOI], [arXiv:0903.4407 [grqc]]. (Cited on page 101.)

[355] Mester, J., Torii, R., Worden, P., Lockerbie, N., Vitale, S. and Everitt, C.W.F., "The STEP mission: principles and baseline design", Class. Quantum Grav., 18, 2475-2486, (2001). [DOI]. (Cited on page 19.)

[356] Mohr, P.J., Taylor, B.N. and Newell, D.B., "CODATA Recommended Values of the Fundamental Physical Constants: 2006", Rev. Mod. Phys., 80, 633, (2008). [DOI], [arXiv:0801.0028 [physics.atom-ph]]. (Cited on page 8.)

[357] Molaro, P., "Newspectrographs for the VLT and E-ELT suited for the measurements of fundamental constant variability", Mem. Soc. Astron. Ital., 80, 912, (2009). (Cited on page 60.)

[358] Molaro, P., Levshakov, S.A. and Kozlov, M.G., "Stringent bounds to spatial variations of the electron-to-proton mass ratio in the Milky Way", Nucl. Phys. B (Proc. Suppl.), 194, 287-293, (2009). [DOI], [arXiv:0907.1192 [astro-ph.CO]]. (Cited on page 105.) 
[359] Molaro, P., Levshakov, S.A., Monai, S., Centurion, M., Bonifacio, P., D’Odorico, S. and Monaco, L., "UVES radial velocity accuracy from asteroid observations. Implications for the fine structure constant variability", Astron. Astrophys., 481, 559, (2008). [arXiv:0712.3345 [astro-ph]]. (Cited on page 51.)

[360] Molaro, P., Murphy, M.T. and Levshakov, S.A., "Exploring variations in the fundamental constants with ELTs: The CODEX spectrograph on OWL", in Whitelock, P.A., Dennefeld, M. and Leibundgut, B., eds., The Scientific Requirements for Extremely Large Telescopes, Proceedings of IAU Symposium 232, Cape Town, South Africa, November 14-18, 2005, IAU Symposia, 232, pp. 198-203, (Cambridge University Press, Cambridge; New York, 2006). [astro-ph/0601264]. (Cited on page 60.)

[361] Molaro, P., Reimers, D., Agafonova, I.I. and Levshakov, S.A., "Bounds on the fine structure constant variability from Fe II absorption lines in QSO spectra", Eur. Phys. J. Special Topics, 163, 173-189, (2008). [DOI], [arXiv:0712.4380 [astro-ph]]. (Cited on pages 50, 51, and 59.)

[362] Moss, A., Scott, D., Zibin, J.P. and Battye, R., "Tilted Physics: A Cosmologically DipoleModulated Sky", arXiv, e-print, (2010). [arXiv:1011.2990 [astro-ph.CO]]. (Cited on page 107.)

[363] Mota, D.F. and Barrow, J.D., "Local and global variations of the fine structure constant", Mon. Not. R. Astron. Soc., 349, 291, (2004). [DOI], [astro-ph/0309273]. (Cited on page 106.)

[364] Müller, C.M., Schäfer, G. and Wetterich, C., "Nucleosynthesis and the variation of fundamental couplings", Phys. Rev. D, 70, 083504, (2004). [DOI], [astro-ph/0405373]. (Cited on pages $71,73,74$, and 94.)

[365] Müller, J., Schneider, M., Soffel, M. and Ruder, H., "Testing Einstein's theory of gravity by analyzing Lunar Laser Ranging data", Astrophys. J., 382, L101, (1991). [DOI]. (Cited on pages 19 and 77 .)

[366] Murphy, M.T., Flambaum, V.V., Muller, S. and Henkel, C., "Strong limit on a variable proton-to-electron mass ratio from molecules in the distant universe", Science, 320, 1611, (2008). [DOI], [arXiv:0806.3081 [astro-ph]]. (Cited on pages 57 and 59.)

[367] Murphy, M.T., Flambaum, V.V., Webb, J.K., Dzuba, V.V., Prochaska, J.X. and Wolfe, A.M., "Constraining Variations in the Fine-structure Constant, Quark Masses and the Strong Interaction", in Karshenboim, S.G. and Peik, E., eds., Astrophysics, Clocks and Fundamental Constants, 302nd WE-Heraeus-Seminar, Bad Honnef, Germany, June 2003, Lecture Notes in Physics, 648, pp. 131-150, (Springer, Berlin, 2004). [DOI], [astro-ph/0310318]. (Cited on pages $45,48,50,51$, and 59.)

[368] Murphy, M.T., Tzanavaris, P., Webb, J.K. and Lovis, C., "Selection of ThAr lines for wavelength calibration of echelle spectra and implications for variations in the fine-structure constant", Mon. Not. R. Astron. Soc., 378, 221, (2007). [DOI], [astro-ph/0703623]. (Cited on pages 48 and 49.)

[369] Murphy, M.T., Webb, J.K. and Flambaum, V.V., "Further evidence for a variable finestructure constant from Keck/HIRES QSO absorption spectra", Mon. Not. R. Astron. Soc., 345, 609, (2003). [DOI], [astro-ph/0306483]. (Cited on page 48.)

[370] Murphy, M.T., Webb, J.K. and Flambaum, V.V., "Comment on 'Limits on the time variation of the electromagnetic fine-structure constant in the low energy Limit from absorption lines in the spectra of distant quasars"', Phys. Rev. Lett., 99, 239001, (2007). [DOI], [arXiv:0708.3677 [astro-ph]]. (Cited on page 50.) 
[371] Murphy, M.T., Webb, J.K. and Flambaum, V.V., "Revision of VLT/UVES constraints on a varying fine-structure constant", Mon. Not. R. Astron. Soc., 384, 1053, (2008). [DOI], [astro-ph/0612407]. (Cited on page 50.)

[372] Murphy, M.T., Webb, J.K. and Flambaum, V.V., "Revisiting VLT/UVES constraints on a varying fine-structure constant", in Santos, N.C., Pasquini, L., Correia, A.C.M. and Romaniello, M., eds., Precision Spectroscopy in Astrophysics, Proceedings of the ESO/Lisbon/Aveiro Conference held in Aveiro, Portugal, 11-15 September 2006, ESO Astrophysics Symposia, pp. 95-100, (Springer, Berlin, 2008). [astro-ph/0611080]. (Cited on page 50.)

[373] Murphy, M.T., Webb, J.K. and Flambaum, V.V., "Keck constraints on a varying finestructure constant: wavelength calibration erros", Mem. Soc. Astron. Ital., 80, 833, (2009). [arXiv:0911.4512 [astro-ph.CO]]. (Cited on page 50.)

[374] Murphy, M.T., Webb, J.K., Flambaum, V.V., Churchill, C.W. and Prochaska, J.X., "Possible evidence for a variable fine-structure constant from QSO absorption lines: systematic errors", Mon. Not. R. Astron. Soc., 327, 1223-1236, (2001). [DOI], [astro-ph/0012420]. (Cited on pages 48 and 49.)

[375] Murphy, M.T., Webb, J.K., Flambaum, V.V., Drinkwater, M.J., Combes, F. and Wiklind, T., "Improved constraints on possible variation of physical constants from $\mathrm{H}$ I $21 \mathrm{~cm}$ and molecular QSO absorption lines", Mon. Not. R. Astron. Soc., 327, 1244-1248, (2001). [DOI], [astro-ph/0101519]. (Cited on pages 53 and 59.)

[376] Murphy, M.T., Webb, J.K., Flambaum, V.V., Dzuba, V.A., Churchill, C.W., Prochaska, J.X., Barrow, J.D. and Wolfe, A.M., "Possible evidence for a variable fine-structure constant from QSO absorption lines: motivations, analysis and results", Mon. Not. R. Astron. Soc., 327, 1208-1222, (2001). [DOI], [astro-ph/0012419]. (Cited on pages 48 and 49.)

[377] Murphy, M.T., Webb, J.K., Flambaum, V.V., Prochaska, J.X. and Wolfe, A.M., "Further constraints on variation of the fine-structure constant from alkali-doublet QSO absorption lines", Mon. Not. R. Astron. Soc., 327, 1237-1243, (2001). [DOI], [astro-ph/0012421]. (Cited on pages 47,59 , and 106.$)$

[378] Nagata, R., Chiba, T. and Sugiyama, N., "WMAP constraints on scalar-tensor cosmology and the variation of the gravitational constant", Phys. Rev. D, 69, 083512, (2004). [DOI]. (Cited on page 83.)

[379] Nakamura, K. et al. (Particle Data Group), "Review of particle physics", J. Phys. G: Nucl. Part. Phys., 37, 075021, (2010). [DOI]. (Cited on pages 5, 10, 12, and 29.)

[380] Nakashima, M., Ichikawa, K., Nagata, R. and Yokoyama, J., "Constraining the time variation of the coupling constants from cosmic microwave background: effect of $\Lambda_{\mathrm{QCD}}$ ", J. Cosmol. Astropart. Phys., 2010(01), 030, (2010). [DOI], [arXiv:0810.1098 [astro-ph]]. (Cited on pages 66 and 67.)

[381] Nakashima, M., Nagata, R. and Yokoyama, J., "Constraints on the time variation of the fine structure constant by the 5yr WMAP data", Prog. Theor. Phys., 120, 1207, (2008). [DOI], [arXiv:0810.1098 [astro-ph]]. (Cited on pages 66 and 67.)

[382] Naudet, R., Oklo, des réacteurs nucléaires fossiles: étude physique, Série Synthèses, (CEA/Eyrolles, Paris, 2000). (Cited on pages 34 and 36.) 
[383] Neal, R.M., Puzzles of Anthropic Reasoning Resolved Using Full Non-indexical Conditioning, Technical Reports, 0607, (University of Toronto, Toronto, 2006). [math/0608592]. Online version (accessed 21 March 2011):

http://www.utstat.utoronto.ca/pagecontent/index.php?pageid=18. (Cited on pages 111 and 113.)

[384] Nguyen, A.T., Budker, D., Lamoreaux, S.K. and Torgerson, J.R., "Towards a sensitive search for variation of the fine-structure constant using radio-frequency $E 1$ transitions in atomic dyprosium", Phys. Rev. A, 69, 022105, (2004). [DOI], [physics/0308104]. (Cited on page 31.)

[385] Nollet, K.M. and Lopez, R.E., "Primordial nucleosynthesis with a varying fine structure constant: an improved estimate", Phys. Rev. D, 66, 063507, (2002). [DOI], [astro-ph/0204325]. (Cited on page 71.)

[386] Nordtvedt, K., " $\dot{G} / G$ and a cosmological acceleration of gravitationally compact bodies", Phys. Rev. Lett., 65, 953-956, (1990). [DOI]. (Cited on pages 21, 77, and 78.)

[387] Noterdaeme, P., Petitjean, P., Srianand, R., Ledoux, C. and Le Petit, F., "Physical conditions in the neutral interstellar medium at $z=2.43$ toward Q2348-011", Astron. Astrophys., 469, 425-436, (2007). [DOI], [astro-ph/0703218]. (Cited on pages 55 and 57.)

[388] Novikov, I.D. and Zel'dovich, Y.B., Relativistic Astrophysics: The structure and evolution of the universe, 2, (University of Chicago Press, Chicago, 1983). (Cited on page 17.)

[389] Nunes, N.J., Dent, T., Avelino, C.J.A.P. and Robbers, G., "Reconstructing the evolution of dark energy with variations of fundamental parameters", Mem. Soc. Astron. Ital., 80, 785, (2009). [arXiv:0910.4935 [astro-ph.CO]]. (Cited on pages 25 and 102.)

[390] Oberhummer, H., Csótó, A. and Schlattl, H., "Stellar production rates of carbon and its abundance in the universe", Science, 289, 88-90, (2000). [DOI], [astro-ph/0007178]. (Cited on page 62.)

[391] Oberhummer, H., Csótó, A. and Schlattl, H., "Bringing the mass gaps at $A=5$ and $A=8$ in nucleosynthesis", Nucl. Phys. A, 689, 269-279, (2001). [DOI], [nucl-th/0009046]. (Cited on page 62.)

[392] Okun, L.B., "The fundamental constants of physics", Sov. Phys. Usp., 34, 818-826, (1991). [DOI]. (Cited on page 16.)

[393] Okun, L.B., "Fundamental Constants of Nature", e-print, (1996). [hep-ph/9612249]. (Cited on page 9.)

[394] Olive, K.A., "The effects of coupling variations on BBN", Mem. Soc. Astron. Ital., 80, 802, (2009). (Cited on page 73.)

[395] Olive, K.A., "Variable constants - A theoretical overview", Mem. Soc. Astron. Ital., 80, 754, (2009). (Cited on page 8.)

[396] Olive, K.A., Peloso, M. and Uzan, J.-P., "The Wall of Fundamental Constants", Phys. Rev. D, 83, 043509, (2011). [DOI], [arXiv:1011.1504 [astro-ph.CO]]. (Cited on page 107.)

[397] Olive, K.A. and Pospelov, M., "Evolution of the Fine Structure Constant Driven by Dark Matter and the Cosmological Constant", Phys. Rev. D, 65, 085044, (2002). [DOI], [hepph/0110377]. (Cited on page 100.) 
[398] Olive, K.A. and Pospelov, M., "Environmental dependence of masses and coupling constants", Phys. Rev. D, 77, 043524, (2008). [DOI], [arXiv:0709.3825 [hep-ph]]. (Cited on page 99.)

[399] Olive, K.A., Pospelov, M., Qian, Y.-Z., Coc, A., Cassé, M. and Vangioni-Flam, E., "Constraints on the variation of the fundamental couplings", Phys. Rev. D, 66, 045022, (2002). [DOI], [hep-ph/0205269]. (Cited on pages 39, 42, 43, and 44.)

[400] Olive, K.A., Pospelov, M., Qian, Y.-Z., Manhès, G., Vangioni-Flam, E., Coc, A. and Cassé, M., "Reexamination of the ${ }^{187}$ Re bound on the variation of fundamental couplings", Phys. Rev. D, 69, 027701, (2004). [DOI], [astro-ph/0309252]. (Cited on page 44.)

[401] Olive, K.A. and Skillman, E.D., "A Realistic Determination of the Error on the Primordial Helium Abundance: Steps Toward Non-Parametric Nebular Helium Abundances", Astrophys. J., 617, 29, (2004). [DOI], [astro-ph/0405588]. (Cited on page 84.)

[402] Page, D.N., "Predictions and tests of multiverse theories", in Carr, B.J., ed., Universe or Multiverse?, pp. 411-430, (Cambridge University Press, Cambridge; New York, 2007). [hepth/0610101]. (Cited on page 113.)

[403] Palma, G.A., Brax, P., Davis, A.C. and van de Bruck, C., "Gauge coupling variation in brane models", Phys. Rev. D, 68, 123519, (2003). [DOI], [astro-ph/0306279]. (Cited on page 101.)

[404] Parkinson, D., Bassett, B.A. and Barrow, J.D., "Mapping the dark energy with varying alpha", Phys. Lett. B, 578, 235, (2004). [astro-ph/0307227]. (Cited on pages 24, 25, and 102.)

[405] Peebles, P.J.E., "Recombination of the primeval plasma", Astrophys. J., 153, 1-11, (1968). [DOI]. (Cited on page 64.)

[406] Peebles, P.J. and Dicke, R.H., "Cosmology and the Radioactive Decay Ages of Terrestrial Rocks and Meteorites", Phys. Rev., 128, 2006-2011, (1962). [DOI]. (Cited on page 43.)

[407] Peik, E., Lipphardt, B., Schnatz, H., Schneider, T. and Tamm, C., "Limit on the Present Temporal Variation of the Fine Structure Constant", Phys. Rev. Lett., 93, 170801, (2004). [DOI], [physics/0402132]. (Cited on pages 29 and 31.)

[408] Peik, E., Lipphardt, B., Schnatz, H., Tamm, C., Weyers, S. and Wynands, R., "Laboratory Limits on Temporal Variations of Fundamental Constants: An Update", in Kleinert, H., Jantzen, R.T. and Ruffini, R., eds., The Eleventh Marcel Grossmann Meeting on General Relativity, Proceedings of the MG11 Meeting on General Relativity, Berlin, Germany, 23-29 July 2006, pp. 941-951, (World Scientific, Singapore; Hackensack, NJ, 2008). [physics/0611088]. (Cited on pages 29, 30, and 105.)

[409] Peter, P. and Uzan, J.-P., Primordial Cosmology, (Oxford University Press, Oxford; New York, 2009). (Cited on pages 22, 24, 63, 68, 69, 88, 106, and 117.)

[410] Petitjean, P. and Aracil, B., "The ratio of the C IV $\lambda \lambda 1548,1550$ rest-wavelengths from highredshift QSO absorption lines", Astron. Astrophys., 422, 523-526, (2004). [DOI]. (Cited on page 47.)

[411] Petitjean, P., Noterdaeme, P., Srianand, R., Ledoux, C., Ivanchik, A. and Gupta, N., "Searching for places where to test the variations of fundamental constants", Mem. Soc. Astron. Ital., 80, 859-863, (2009). [ADS]. (Cited on pages 52 and 56.) 
[412] Petitjean, P., Srianand, R., Chand, H., Ivanchik, A., Noterdaeme, P. and Gupta, N., "Constraining fundamental constants of physics with quasar absorption line systems", Space Sci. Rev., 148, 289-300, (2009). [DOI], [arXiv:0905.1516 [astro-ph.CO]]. (Cited on pages 44, 57, and 59.)

[413] Petley, B.W., "New definition of the metre", Nature, 303, 373, (1983). [DOI]. (Cited on page 11.)

[414] Petley, B.W., The Fundamental Physical Constants and the Frontier of Measurement, (Adam Hilger, Bristol; Philadelphia, 1985). (Cited on page 13.)

[415] Petrov, Y.V., "The Oklo natural reactor", Sov. Phys. Usp., 20, 937, (1978). (Cited on pages 37 and 38.)

[416] Petrov, Y.V., Nazarov, A.I., Onegin, M.S., Petrov, V.Y. and Sakhnovsky, E.G., "Natural nuclear reactor at Oklo and variation of fundamental constants: Computation of neutronics of a fresh core", Phys. Rev. C, 74, 064610, (2006). [DOI], [hep-ph/0506186]. (Cited on pages $37,38,39$, and 40.$)$

[417] Pitrou, C., Uzan, J.-P. and Bernardeau, F., "Cosmic microwave background bispectrum on small angular scales", Phys. Rev. D, 78, 063526, (2008). [DOI], [arXiv:0807.0341 [astro-ph]]. (Cited on page 106.)

[418] Planck, M., "Über irreversible Strahlungsvorgänge", Ann. Phys. (Berlin), 1, 69, (1900). [DOI]. (Cited on page 16.)

[419] Planck, M., "Natural Units", in The Theory of Heat Radiation, pp. 173-175, (P. Blakiston's Son \& Co., Philadelphia, 1914). [Google Books]. (Cited on page 16.)

[420] Polchinski, J., String Theory. Vol. 2: Superstring theory and beyond, Cambridge Monographs on Mathematical Physics, (Cambridge University Press, Cambridge; New York, 1998). [Google Books]. (Cited on page 89.)

[421] Porsev, S.G., Flambaum, V.V. and Torgerson, J.R., "Transition frequency shifts with fine-structure constant variation for Yb II", Phys. Rev. A, 80, 042503, (2009). [DOI], [arXiv:0907.3352 [physics.atom-ph]]. (Cited on page 33.)

[422] Prestage, J.D., Bollinger, J.J., Itano, W.M. and Wineland, D.J., "Limits for Spatial Anisotropy by Use of Nuclear-Spin-Polarized ${ }^{9} \mathrm{Be}^{+}$Ions", Phys. Rev. Lett., 54, 2387-2390, (1985). [DOI]. (Cited on page 18.)

[423] Pretage, J.D., Tjoelker, R.L. and Maleki, L., "Atomic clocks and variation of the fine structure constant", Phys. Rev. Lett., 74, 3511-3514, (1995). [DOI]. (Cited on page 28.)

[424] Prunet, S., Uzan, J.-P., Bernardeau, F. and Brunier, T., "Constraints on mode couplings and modulation of the CMB with WMAP data", Phys. Rev. D, 71, 083508, (2005). [DOI], [arXiv:astro-ph/0406364]. (Cited on page 107.)

[425] Psaltis, D., "Probes and Tests of Strong-Field Gravity with Observations in the Electromagnetic Spectrum", Living Rev. Relativity, 11, lrr-2008-9, (2008). [arXiv:0806.1531 [astro-ph]]. URL (accessed 27 September 2010):

http://www.livingreviews.org/lrr-2008-9. (Cited on page 20.)

[426] Pudliner, B.S., Pandharipande, V.R., Carlson, J. and Pieper, S.C. etal, "Quantum Monte Carlo calculations of nuclei with $A \leq 7$ ", Phys. Rev. C, 56, 1720, (1997). [DOI], [nuclth/9705009]. (Cited on pages 74 and 95.) 
[427] Quast, R., Reimers, D. and Levshakov, S.A., "Probing the variability of the fine-structure constant with the VLT/UVES", Astron. Astrophys., 415, L7-L11, (2004). [DOI], [astroph/0311280]. (Cited on pages 50 and 51.)

[428] Reasenberg, R.D., "The constancy of $G$ and other gravitational experiments", Philos. Trans. R. Soc. London, Ser. A, 310, 227, (1983). (Cited on page 77.)

[429] Reasenberg, R.D. and Shapiro, I.I., "A radar test of the constancy of the gravitational interaction", in Halpern, L., ed., On the Measurement of Cosmological Variations of the Gravitational Constant, Workshop meeting held at Florida State University, Tallahassee, November 12-14, 1975, pp. 71-86, (University Presses of Florida, Gainesville, FL, 1978). (Cited on page 77.)

[430] Reasenberg, R.D. et al., "Viking relativity experiment: Verification of signal retardation by solar gravity", Astrophys. J. Lett., 234, L219-L221, (1979). [DOI], [ADS]. (Cited on page 77.)

[431] Reinhold, E., Buning, R., Hollenstein, U., Ivanchik, A., Petitjean, P. and Ubachs, W., "Indication of a Cosmological Variation of the Proton-Electron Mass Ratio Based on Laboratory Measurement and Reanalysis of $\mathrm{H}_{2}$ spectra", Phys. Rev. Lett., 96, 151101, (2006). [DOI]. (Cited on pages 56 and 59.)

[432] Reisenegger, E., Jofré, P. and Fernandez, R., "Constraining a possible time-variation of the gravitational constant through 'gravitochemical heating' of neutron stars", Mem. Soc. Astron. Ital., 80, 829, (2009). [arXiv:0911.0190 [astro-ph.HE]]. (Cited on page 79.)

[433] Reynaud, S., Salomon, C. and Wolf, P., "Testing general relativity with atomic clocks", Space Sci. Rev., 148, 233-247, (2009). [DOI], [arXiv:0903.1166 [quant-ph]]. (Cited on page 34.)

[434] Riazuelo, A. and Uzan, J.-P., "Quintessence and gravitational waves", Phys. Rev. D, 62, 083506, (2000). [DOI]. (Cited on page 23.)

[435] Riazuelo, A. and Uzan, J.-P., "Cosmological observations in scalar-tensor quintessence", Phys. Rev. D, 66, 023525, (2002). [DOI], [astro-ph/0107386]. (Cited on pages 24, 64, 81, 82, 83, 87, and 106.)

[436] Ricci, B. and Villante, F.L., "The Sun and the Newton Constant", Phys. Lett. B, 549, 20, (2002). [astro-ph/0204482]. (Cited on page 80.)

[437] Rich, J., "Experimental Consequences of Time Variations of the Fundamental Constants", Am. J. Phys., 71, 1043, (2003). [DOI], [physics/0209016]. (Cited on page 17.)

[438] Rocha, G., Trotta, R., Martins, C.J.A.P., Melchiorri, A., Avelino, P.P., Bean, R. and Viana, P.T.P., "Measuring $\alpha$ in the early universe: CMB polarisation, reionisation and the Fisher matrix analysis", Mon. Not. R. Astron. Soc., 352, 20-38, (2004). [DOI], [ADS], [astro-ph/0309211]. (Cited on pages 66 and 67.)

[439] Rohlf, J.W., Modern Physics from a to Z , (Wiley, New York, 1994). (Cited on page 95.)

[440] Rosenband, T. et al., "Frequency Ratio of $\mathrm{Al}^{+}$and $\mathrm{Hg}^{+}$Single-Ion Optical Clocks; Metrology at the 17th Decimal Place", Science, 319, 1808-1812, (2008). [DOI]. (Cited on pages 29, 31, and 105.)

[441] Rothman, T. and Matzner, R., "Scale-Covariant Gravitation and Primordial Nucleosynthesis", Astrophys. J., 257, 450, (1982). [DOI]. (Cited on pages 83 and 84.) 
[442] Rozental, I.L., Big Bang, Big Bounce: How Particles and Fields Drive Cosmic Evolution, (Springer, Berlin, 1988). (Cited on page 111.)

[443] Rubakov, V.A. and Shaposhnikov, M.E., "A Comment on Dynamical Coupling Constants and the Anthropic Principle", Mod. Phys. Lett. A, 4, 107-109, (1989). [DOI]. (Cited on page 113.)

[444] Salomon, C. et al., "Cold atoms in space and atomic clocks: ACES", C. R. Acad. Sci., 2, 1313-1330, (2001). [DOI]. (Cited on pages 34 and 105.)

[445] Sandvik, H.B., Barrow, J.D. and Magueijo, J., "A Simple Cosmology with a Varying Fine Structure Constant", Phys. Rev. Lett., 88, 031302, (2002). [DOI], [astro-ph/0107512]. (Cited on page 100.)

[446] Santiago, D.I., Kalligas, D. and Wagoner, R.V., "Nucleosynthesis constraints on scalar-tensor theories of gravity", Phys. Rev. D, 56, 7627-7637, (1997). [DOI]. (Cited on page 83.)

[447] Savedoff, M., "Physical constants in extra-galactic nebulae", Nature, 178, 688, (1956). [DOI]. (Cited on page 44.)

[448] Schiller, S., "Hydrogenlike highly charged ions tests of the time independence of fundamental constants", Phys. Rev. Lett., 98, 180801, (2007). [DOI]. (Cited on page 33.)

[449] Schimd, C., Uzan, J.-P. and Riazuelo, A., "Weak lensing in scalar-tensor theories of gravity", Phys. Rev. D, 71, 083512, (2005). [DOI], [astro-ph/0412120]. (Cited on page 87.)

[450] Schlamminger, S., Choi, K.-Y., Wagner, T.A., Gundlach, J.H. and Adelberger, E.G., "Test of the Equivalence Principle Using a Rotating Torsion Balance", Phys. Rev. Lett., 100, 041101, (2008). [DOI]. (Cited on page 19.)

[451] Schlattl, H., Heger, A., Oberhummer, H., Rauscher, T. and Csoto, A., "Sensitivity of the C and O production on the $3 \alpha$ rate", Astrophys. Space Sci., 291, 27, (200). [astro-ph/0307528]. (Cited on page 62.)

[452] Scoccola, C.G., Landau, S.J. and Vucetich, H., "WMAP-5yr constraints on time variation of $\alpha$ and $m_{e}$ in a detailed recombination scenario", Phys. Lett. B, 669, 212, (2008). [arXiv:0809.5028 [astro-ph]]. (Cited on pages 66 and 67.)

[453] Scoccola, C.G., Landau, S.J. and Vucetich, H., "WMAP 5-year constraints on time variation of $\alpha$ and $m_{e}$ ", Mem. Soc. Astron. Ital., 80, 814, (2009). [arXiv:0910.1083 [astro-ph.CO]]. (Cited on pages 66 and 67.)

[454] Seager, S., Savelov, D.D. and Scott, D., "A new calculation of the recombination epoch", Astrophys. J. Lett., 523, L1, (1999). [DOI], [astro-ph/9909275]. (Cited on page 65.)

[455] Serna, A. and Alimi, J.M., "Scalar-tensor cosmological models", Phys. Rev. D, 53, 3074, (1996). [DOI]. (Cited on page 83.)

[456] Serot, B.D. and Walecka, J.D., "Recent progress in quantum hadrodynamics", Int. J. Mod. Phys. E, 6, 515, (1997). [DOI]. (Cited on page 95.)

[457] Shapiro, I.I., "Solar system tests of general relativity: Recent results and present plans", in Ashby, N., Bartlett, D.F. and Wyss, W., eds., General Relativity and Gravitation, Proceedings of the 12th International Conference on General Relativity and Gravitation, University of Colorado at Boulder, July 2-8, 1989, pp. 313-330, (Cambridge University Press, Cambridge; New York, 1990). [Google Books]. (Cited on pages 20 and 77.) 
[458] Shapiro, I.I., Smith, W.B. and Ash, M.B., "Gravitational Constant: Experimental Bound on Its Time Variation", Phys. Rev. Lett., 26, 27, (1971). [DOI]. (Cited on page 77.)

[459] Shapiro, S.S., Davis, J.L., Lebach, D.E. and Gregory, J.S., "Measurement of the Solar Gravitational Deflection of Radio Waves using Geodetic Very-Long-Baseline Interferometry Data, 1979-1999", Phys. Rev. Lett., 92, 121101, (2004). [DOI]. (Cited on page 20.)

[460] Shaw, D.J., "Detecting Seasonal Changes in the Fundamental Constants", e-print, (2007). [gr-qc/0702090]. (Cited on page 104.)

[461] Shaw, D.J. and Barrow, J.D., "Varying couplings in electroweak theory", Phys. Rev. D, 71, 063525, (2005). [DOI], [gr-qc/0412135]. (Cited on pages 100 and 104.)

[462] Shaw, D.J. and Barrow, J.D., "Local Experiments See Cosmologically Varying Constants", Phys. Lett. B, 639, 596-599, (2006). [gr-qc/0512117]. (Cited on page 104.)

[463] Shaw, D.J. and Barrow, J.D., "Varying constants: constraints from seasonal variations", Mem. Soc. Astron. Ital., 80, 791, (2009). (Cited on pages 105 and 109.)

[464] Shelnikov, A., Butcher, R.J., Chardonnet, C. and Amy-Klein, A., "Stability of the protonto-electron mass ratio", Phys. Rev. Lett., 100, 150801, (2008). [DOI], [arXiv:0803.1829 [physics.atom-ph]]. (Cited on page 32.)

[465] Shlyakhter, A.I., "Direct test of the constancy of the fundamental constants using Oklo nuclear reactor", Nature, 264, 340, (1976). [DOI]. (Cited on pages 35, 36, 37, and 38.)

[466] Sigurdson, K., Kurylov, A. and Kamionkowski, M., "Spatial Variation of the Fine-Structure Parameter and the Cosmic Microwave Background", Phys. Rev. D, 68, 103509, (2003). [DOI], [astro-ph/0306372]. (Cited on page 106.)

[467] Sisterna, P. and Vucetich, H., "Time variation of fundamental constants: bounds from geophysical and astronomical data", Phys. Rev. D, 41, 1034, (1990). [DOI]. (Cited on pages 39 and 40.)

[468] Smoliar, M., Walker, R. and Morgan, J., "Re-Os ages from group IIA, IIIA, IVA and IVB iron meteorites", Science, 271, 1099, (1996). [DOI]. (Cited on page 43.)

[469] Spite, M. and Spite, F., "Li isotopes in metal-poor halo dwarfs, a more and more complicated story", in Charbonnel, C., Tosi, M., Primas, F. and Chiappini, C., eds., Light Elements in the Universe, Proceedings of IAU Symposium 268, Geneva, Switzerland, 9 -13 November 2009, IAU Symposia, 268, pp. 201-210, (Cambridge University Press, Cambridge; New York, 2009). [DOI], [arXiv:1002.1004 [astro-ph.GA]]. (Cited on page 70.)

[470] Srianand, R., Chand, H., Petitjean, P. and Aracil, B., "Limits on the time variation of the electromagnetic fine-structure constant in the low energy limit from absorption lines in the spectra of distant quasars", Phys. Rev. Lett., 92, 121302, (2004). [DOI], [astro-ph/0402177]. (Cited on pages 49 and 50.)

[471] Srianand, R., Chand, H., Petitjean, P. and Aracil, B., "Reply to the Comment by M.T. Murphy, J.K. Webb, and V.V. Flambaum", Phys. Rev. Lett., 99, 239002, (2007). [DOI], [arXiv:0711.1742 [astro-ph]]. (Cited on pages 50 and 51.)

[472] Srianand, R., Gupta, N., Petitjean, P., Noterdaeme, P. and Ledoux, C., "Detection of 21-cm, $\mathrm{H}_{2}$ and Deuterium absorption at $z>3$ along the line of sight to J1337+3152", Mon. Not. R. Astron. Soc., 405, 1888-1900, (2010). [DOI], [arXiv:1002.4620 [astro-ph.CO]]. (Cited on pages 52 and 59.) 
[473] Srianand, R., Noterdaeme, P., Ledoux, C. and Petitjean, P., "First detection of CO in a high-redshift damped Lyman- $\alpha$ system", Astron. Astrophys., 482, L39-L42, (2008). [DOI]. (Cited on page 57.)

[474] Srianand, R., Petitjean, P., Chand, H., Noterdaeme, P. and Gupta, N., "Probing the variation of fundamental constants using QSO absorption lines", Mem. Soc. Astron. Ital., 80, 842, (2009). (Cited on page 44.)

[475] Starkman, G.D. and Trotta, R., "Why anthropic reasoning cannot predict $\Lambda$ ", Phys. Rev. Lett., 97, 201301, (2006). [DOI], [astro-ph/0607227]. (Cited on page 113.)

[476] Stefanecsu, P., "Constraints on time variation of the fine structure constant from WMAP3yr data", New Astronomy, 12, 635, (2007). [arXiv:0707.0190 [astro-ph]]. (Cited on pages 66 and 67.)

[477] Steinhardt, P.J. and Wesley, D., "Exploring extra dimensions through observational tests of dark energy and varying Newton's constant", arXiv, e-print, (2010). [arXiv:1003.2815 [astroph.CO]]. (Cited on page 101.)

[478] Steinmetz, T. et al., "Laser frequency combs for astronomical observations", Science, 321, 1335-1337, (2008). [DOI], [arXiv:0809.1663 [astro-ph]]. (Cited on page 60.)

[479] Stoeger, W.R., "Retroduction, Multiverse Hypotheses and Their Testability", e-print, (2006). [astro-ph/0602356]. (Cited on page 113.)

[480] Stoeger, W.R., Ellis, G.F.R. and Kirchner, U., "Multiverses and Cosmology: Philosophical Issues", arxiv, e-print, (2004). [arxiv:astro-ph/0407329]. (Cited on page 113.)

[481] Su, Y., Heckel, B.R., Adelberger, E.G., Gundlach, J.H., Harris, M., Smith, G.L. and Swanson, H.E., "New tests of the universality of free fall", Phys. Rev. D, 50, 3614-3636, (1994). [DOI]. (Cited on page 19.)

[482] Susskind, L., "The Anthropic Landscape of String Theory", e-print, (2003). [hep-th/0302219]. (Cited on page 110.)

[483] Taveras, V. and Yunes, N., "The Barbero-Immirzi parameter as a scalar field: $K$-inflation from loop quantum gravity?", Phys. Rev. D, 78, 064070, (2008). [DOI], [arXiv:0807.2652 [grqc]]. (Cited on page 101.)

[484] Taylor, T.R. and Veneziano, G., "Dilaton couplings at large distance", Phys. Lett. B, 213, 450-454, (1988). [DOI]. (Cited on pages 89 and 90.)

[485] Tegmark, M., Aguirre, A., Rees, M.J. and Wilczek, F., "Dimensionless constants, cosmology and other dark matters", Phys. Rev. D, 73, 023505, (2006). [DOI], [astro-ph/0511774]. (Cited on page 112.)

[486] Tegmark, M. and Rees, M.J., "Why is the level fluctuation level 10-5?", Astrophys. J., 499, 526, (1998). [astro-ph/9709058]. (Cited on page 112.)

[487] Teller, E., "On The Change Of Physical Constants", Phys. Rev., 73, 801, (1948). [DOI]. (Cited on page 79.)

[488] Thompson, R.I., "The determination of the electron-to-proton inertial mass via molecular transitions", Astrophys. Lett., 16, 3, (1975). (Cited on pages 32 and 55.) 
[489] Thompson, R.I., "Observational determinations of the proton to electron mass ratio in the early universe", Mem. Soc. Astron. Ital., 80, 870, (2009). (Cited on page 56.)

[490] Thompson, R.I. et al., "An observational determination of the proton to electron mass ratio in the early universe", Astrophys. J., 703, 1648, (2009). [DOI], [arXiv:0907.4392 [astro-ph.CO]]. (Cited on pages 56 and 59.)

[491] Thomson, D.R., LeMere, M. and Tang, Y.C., "Systematic investigation of scattering problems with the resonating-group method", Nucl. Phys. A, 286, 53-66, (1977). [DOI]. (Cited on page 62.)

[492] Thorsett, S.E., "The Gravitational Constant, The Chandrasekhar Limit, And Neutron Star Masses", Phys. Rev. Lett., 77, 1432-1435, (1996). [DOI]. (Cited on pages 81 and 82.)

[493] Touboul, P., Rodrigues, M., Métris, G. and Tatry, B., "MICROSCOPE, testing the equivalence principle in space", C. R. Acad. Sci., 2(9), 1271-1286, (2001). (Cited on page 19.)

[494] Tsanavaris, P., Murphy, M.T., Webb, J.K., Flambaum, V.V. and Curran, S.J., "Probing variations in fundamental constants with radio and optical quasar absorption-line observations", Mon. Not. R. Astron. Soc., 374, 634-646, (2007). [DOI], [ADS], [astro-ph/0610326]. (Cited on pages 52 and 59.)

[495] Turyshev, S.G., "Experimental Tests of General Relativity", Annu. Rev. Nucl. Part. Sci., 58, 207-248, (2008). [DOI], [arXiv:0806.1731 [gr-qc]]. (Cited on page 20.)

[496] Tzanavaris, P., Webb, J.K., Murphy, M.T., Flambaum, V.V. and Curran, S.J., "Limits on Variations in Fundamental Constants from 21-cm and Ultraviolet Quasar Absorption Lines", Phys. Rev. Lett., 95, 041301, (2005). [DOI], [astro-ph/0412649]. (Cited on page 52.)

[497] Unzicker, A., "A look at the abandoned contributions to cosmology of Dirac, Sciama and Dicke", Ann. Phys. (Berlin), 18(1), 57, (2009). [DOI], [arXiv:0708.3518 [physics.gen-ph]]. (Cited on page 7.)

[498] Upadhye, A., Gruber, S.S. and Khoury, J., "Unveiling chameleons in tests of gravitational inverse-square law", Phys. Rev. D, 74, 104024, (2006). [DOI], [hep-ph/0608186]. (Cited on page 99.)

[499] Uzan, J.-P., "Cosmological scaling solutions of nonminimally coupled scalar fields", Phys. Rev. D, 59, 123510, (1999). [DOI], [gr-qc/9903004]. (Cited on page 23.)

[500] Uzan, J.-P., "The fundamental constants an their variation: observational and theoretical status", Rev. Mod. Phys., 75, 403-455, (2003). [DOI], [hep-ph/0205340]. (Cited on pages 8, $29,43,44,47,55,66,70,76$, and 79.)

[501] Uzan, J.-P., "Variation of the Constants of Nature in the Early and Late Universe", in Martins, C.J.A.P., Avelino, P.P., Costa, M.S., Mack, K., Mota, M.F. and Parry, M., eds., Phi in the Sky: The Quest for Cosmological Scalar Fields, Porto, Portugal, 8-10 July 2004, AIP Conference Proceedings, 736, pp. 3-20, (American Institute of Physics, Melville, NY, 2004). [DOI], [astro-ph/0409424]. (Cited on page 8.)

[502] Uzan, J.-P., "The acceleration of the universe and the physics behind it", Gen. Relativ. Gravit., 39, 307, (2007). [DOI], [astro-ph/0605313]. (Cited on pages 23 and 82.)

[503] Uzan, J.-P., "Fundamental constants, general relativity and cosmology", Mem. Soc. Astron. Ital., 80, 762, (2009). (Cited on page 8.) 
[504] Uzan, J.-P., "Dark energy, gravitation and the Copernican principle", in Ruiz-Lapuente, P., ed., Dark Energy: Observational and Theoretical Approaches, pp. 3-47, (Cambridge University Press, Cambridge; New York, 2010). [arXiv:0912.5452 [gr-qc]]. (Cited on pages 23 and 82.)

[505] Uzan, J.-P., "Fundamental constants and tests of general relativity - Theoretical and cosmological considerations", Space Sci. Rev., 148, 249, (2010). [arXiv:0907.3081 [gr-qc]]. (Cited on page 8.)

[506] Uzan, J.-P., "Tests of General Relativity on Astrophysical Scales", Gen. Relativ. Gravit., 42, 2219, (2010). [DOI], [arXiv:0908.2243 [astro-ph.CO]]. (Cited on page 82.)

[507] Uzan, J.-P., Bernardeau, F. and Mellier, Y., "Time drift of cosmological redshifts and its variance", Phys. Rev. D, 77, 021301(R), (2008). [DOI], [arXiv:0711.1950 [astro-ph]]. (Cited on page 60.)

[508] Uzan, J.-P., Clarkson, C. and Ellis, G.F.R., "Time drift of cosmological redshifts as a test of the Copernican principle", Phys. Rev. Lett., 100, 191303, (2008). [DOI], [arXiv:0801.0068 [astro-ph]. (Cited on page 23.)

[509] Uzan, J.-P. and Leclercq, B., The Natural Laws of the Universe: Understanding Fundamental Constants, Springer-Praxis Books in Popular Astronomy, (Springer; Praxis, New York; Chichester, UK, 2008). (Cited on pages 9, 14, and 34.)

[510] Uzan, J.-P. and Lehoucq, R., Les constantes fondamentales, (Belin, Paris, 2005). (Cited on pages 9,11 , and 14.)

[511] Vaas, R., "Multiverse Scenarios in Cosmology: Classification, Cause, Challenge, Controversy, and Criticism", J. Cosmol., 4, 664-673, (2010). [arXiv:1001.0726 [physics.gen-ph]]. URL (accessed 25 March 2010):

http://journalof cosmology.com/Multiverse7.html. (Cited on page 113.)

[512] Varshalovich, D.A. and Levshakov, S.A., "On a time dependence of physical constants", J. Exp. Theor. Phys. Lett., 58, 231, (1993). (Cited on page 55.)

[513] Varshalovich, D.A. and Potekhin, A.Y., "Have the masses of molecules changed during the lifetime of the Universe?", Astron. Lett., 22, 1, (1996). (Cited on page 53.)

[514] Veneziano, G., "A Stringy Nature Needs Just Two Constants", Europhys. Lett., 2, 199, (1986). [DOI]. (Cited on page 16.)

[515] Veneziano, G., "Large- $N$ bounds on, and compositeness limit of, gauge and gravitational interactions", J. High Energy Phys., 2002(06), 051, (2002). [DOI], [hep-th/0110129]. (Cited on page 89.)

[516] Verbiest, J.P.W. et al., "Precision timing of PSR J0437-4715: an accurate pulsar distance, a high pulsar mass and a limit on the variation of Newton's gravitational constant", Astrophys. J., 679, 675-680, (2008). [DOI], [arXiv:0801.2589 [astro-ph]]. (Cited on page 78.)

[517] Vessot, R.F.C. and Levine, M.W., "A Test of the Equivalence Principle Using a Space-Borne Clock", Gen. Relativ. Gravit., 10, 181-204, (1979). [DOI]. (Cited on page 19.)

[518] Vila, S.C., "Changing gravitational constant and white dwarfs", Astrophys. J., 206, 213, (1976). [DOI]. (Cited on page 81.) 
[519] Vilenkin, A., "Predictions from Quantum Cosmology", Phys. Rev. Lett., 74, 846-849, (1995). [DOI], [gr-qc/9406010]. (Cited on page 113.)

[520] Volovik, G.E., "Fundamental constants in effective theory", J. Exp. Theor. Phys. Lett., 76, 77, (2002). [DOI], [physics/0203075]. (Cited on page 17.)

[521] Volovik, G.E., " $\hbar$ as parameter of Minkowski metric in effective theory", J. Exp. Theor. Phys. Lett., 90, 697-704, (2009). [DOI], [arXiv:0904.1965 [gr-qc]]. (Cited on page 9.)

[522] Webb, J.K., Flambaum, V.V., Churchill, C.W., Drinkwater, M.J. and Barrow, J.D., "Search for time variation of the fine structure constant", Phys. Rev. Lett., 82, 884-887, (1999). [DOI], [astro-ph/9803165]. (Cited on pages 47 and 48.)

[523] Webb, J.K., King, J.A., Murphy, M.T., Flambaum, V.V., Carswell, R.F. and Bainbridge, M.B., "Evidence for spatial variation of the fine structure constant", arXiv, e-print, (2010). [arXiv:1008.3907 [astro-ph.CO]]. (Cited on pages 51 and 106.)

[524] Webb, J.K., Murphy, M.T., Flambaum, V.V., Dzuba, V.A., Barrow, J.D., Churchill, C.W., Prochaska, J.X. and Wolfe, A.M., "Further Evidence for Cosmological Evolution of the Fine Structure Constant", Phys. Rev. Lett., 87, 091301, (2001). [DOI], [astro-ph/0012539]. (Cited on pages 48 and 89.)

[525] Weinberg, S., "Charges from extra dimensions", Phys. Lett. B, 125, 265-269, (1983). [DOI]. (Cited on page 89.)

[526] Weinberg, S., "Overview of theoretical prospects for understanding the values of fundamental constants", Philos. Trans. R. Soc. London, Ser. A, 310, 249, (1983). [DOI]. (Cited on page 9.)

[527] Weinberg, S., "Anthropic Bound on the Cosmological Constant", Phys. Rev. Lett., 59, 26072610, (1987). [DOI]. (Cited on page 112.)

[528] Weinberg, S., "The cosmological constant problem", Rev. Mod. Phys., 61, 1-23, (1989). [DOI]. (Cited on pages 23 and 113.)

[529] Wendt, M. and Reimers, D., "Variability of the proton-to-electron mass ratio on cosmological scales", Eur. Phys. J. Special Topics, 163, 197-206, (2008). [DOI], [arXiv:0802.1160 [astro-ph]]. (Cited on page 56.)

[530] Wendt, M., Reimers, D. and Molaro, P., "Cosmological observations to shed light on possible variations: expectations, limitations and status quo", Mem. Soc. Astron. Ital., 80, 876-881, (2009). (Cited on page 56.)

[531] Wetterich, C., "Crossover quintessence and cosmological history of fundamental 'constants", Phys. Lett. B, 561, 10, (2003). [hep-ph/0301261]. (Cited on pages 24 and 102.)

[532] Wetterich, C., "Probing Quintessence with Time Variation of Couplings", J. Cosmol. Astropart. Phys., 2003(10), 002, (2003). [DOI], [hep-ph/0203266]. (Cited on pages 24 and 108.)

[533] Wetterich, C., "Growing neutrinos and cosmological selection", Phys. Lett. B, 655, 201-208, (2007). [DOI], [arXiv:0706.4427 [hep-ph]]. (Cited on pages 101 and 102.)

[534] Whitmore, J.B., Murphy, M.T. and Griest, K., "Wavelength Calibration of the VLTUVES Spectrograph", Astrophys. J., 732, 89-99, (2010). [DOI], [arXiv:1004.3325 [astro-ph.IM]]. (Cited on pages 48,49 , and 50 .) 
[535] Wignall, J.W.G., "How many fundamental constants does quantum physics need?", Int. J. Mod. Phys. A, 15, 875, (2000). (Cited on page 16.)

[536] Wiklind, T. and Combes, F., "Molecular absorption lines at high redshift: PKS 1413+135 $(z=0.247)$ ", Astron. Astrophys., 328, 48, (1997). [astro-ph/9708051]. (Cited on pages 53 and 59.)

[537] Wilczek, F., "Enlightenment, Knowledge, Ignorance, Temptation", e-print, (2005). [hepph/0512187]. (Cited on page 113.)

[538] Wilczek, F., "Fundamental constants", arXiv, e-print, (2007). [arXiv:0708.4361 [hep-ph]]. (Cited on pages 9, 11, and 113.)

[539] Wilkinson, D.H., "Do the 'constants of nature' change with time?", Philos. Mag., 3, 582, (1958). [DOI]. (Cited on pages 40 and 42.)

[540] Will, C.M., Theory and Experiment in Gravitational Physics, (Cambridge University Press, Cambridge; New York, 1993), 2nd edition. [Google Books]. (Cited on pages 17, 20, 76, and 85.)

[541] Will, C.M., "The Confrontation between General Relativity and Experiment", Living Rev. Relativity, 9, lrr-2006-3, (2006). [gr-qc/0510072]. URL (accessed 27 September 2010): http://www. livingreviews.org/lrr-2006-3. (Cited on page 20.)

[542] Williams, J.G., Newhall, X.X. and Dickey, J.O., "Relativity parameters determined from lunar laser ranging", Phys. Rev. D, 53, 6730-6739, (1996). [DOI]. (Cited on pages 19 and 77.)

[543] Williams, J.G., Turyshev, S.G. and Boggs, D.H., "Progress in Lunar Laser Ranging Tests of Relativistic Gravity", Phys. Rev. Lett., 93, 261101, (2004). [DOI], [gr-qc/0411113]. (Cited on pages 19, 20, and 77.)

[544] Williams, J.G. et al., "New Test of the Equivalence Principle from Lunar Laser Ranging", Phys. Rev. Lett., 36, 551-554, (1976). [DOI]. (Cited on page 77.)

[545] Witten, E., "Search for a Realistic Kaluza-Klein Theory", Nucl. Phys. B, 186, 412, (1981). [DOI]. (Cited on page 89.)

[546] Witten, E., "Some Properties of O(32) Superstrings", Phys. Lett. B, 149, 351-356, (1984). [DOI], [ADS]. (Cited on pages 89 and 90.)

[547] Wolf, P. et al., "Quantum physics exploring gravity in the outer solar system: the SAGAS project", Exp. Astron., 23, 651-687, (2009). [DOI], [arXiv:0711.0304 [gr-qc]]. (Cited on pages 34 and 105.)

[548] Wolfe, A.M., Broderick, J.J., Condon, J.J. and Johnston, K.J., "3C 286: A cosmological QSO?", Astrophys. J. Lett., 208, L47, (1976). [DOI], [ADS]. (Cited on page 52.)

[549] Wu, F. and Chen, X., "Cosmic microwave background with Brans-Dicke gravity II: constraints with the WMAP and SDSS data", Phys. Rev. D, 82, 083003, 1-10, (2010). [DOI], [arXiv:0903.0385 [astro-ph.CO]]. (Cited on page 83.)

[550] Wu, Y.S. and Wang, Z., "Time Variation of Newton's Gravitational Constant in Superstring Theories", Phys. Rev. Lett., 57, 1978, (1986). [DOI]. (Cited on page 89.) 
[551] Yang, J., Schramm, D.N., Steigmann, G. and Rood, R.T., "Time Variation of Newton's Gravitational Constant in Superstring Theories", Astrophys. J., 227, 697, (1979). (Cited on pages 83 and 84.)

[552] Yong, D., Grundahl, F., Lambert, D.L., Nissen, P.E. and Shetrone, M.D., "Mg isotopic ratios in giant stars of the globular cluster NGC 6752", Astron. Astrophys., 402, 985-1001, (2003). [DOI], [astro-ph/0303057]. (Cited on page 49.)

[553] Yoo, J.J. and Scherrer, R.J., "Big bang nucleosynthesis and cosmic background constraints on the time variation of the Higgs vaccuum expectation value", Phys. Rev. D, 67, 043517, (2003). [DOI], [astro-ph/0211545]. (Cited on pages 74 and 95.)

[554] Yunes, N., Pretorius, F. and Spergel, D., "Constraining the evolutionary history of Newton's constant with gravitational wave observations", Phys. Rev. D, 81, 064018, (2010). [DOI], [arXiv:0912.2724 [gr-qc]]. (Cited on page 82.) 